\title{
CHANNEL PREDICTION FOR MOBILE MIMO WIRELESS COMMUNICATION SYSTEMS
}

BY

\section{RAMONI O. ADEOGUN}

\author{
A thesis \\ submitted to the Victoria University of Wellington \\ in fulfilment of the \\ requirements for the degree of \\ Doctor of Philosophy \\ in Engineering. \\ Victoria University of Wellington \\ 2015
}





\begin{abstract}
7 Emporal variation and frequency selectivity of wireless channels con1 stitute a major drawback to the attainment of high gains in capacity and reliability offered by multiple antennas at the transmitter and receiver of a mobile communication system. Limited feedback and adaptive transmission schemes such as adaptive modulation and coding, antenna selection, power allocation and scheduling have the potential to provide the platform of attaining the high transmission rate, capacity and QoS requirements in current and future wireless communication systems. Theses schemes require both the transmitter and receiver to have accurate knowledge of Channel State Information (CSI). In Time Division Duplex (TDD) systems, CSI at the transmitter can be obtained using channel reciprocity. In Frequency Division Duplex (FDD) systems, however, CSI is typically estimated at the receiver and fed back to the transmitter via a low-rate feedback link. Due to the inherent time delays in estimation, processing and feedback, the CSI obtained from the receiver may become outdated before its actual usage at the transmitter. This results in significant performance loss, especially in high mobility environments. There is therefore a need to extrapolate the varying channel into the future, far enough to account for the delay and mitigate the performance degradation.

The research in this thesis investigates parametric modeling and prediction of mobile MIMO channels for both narrowband and wideband systems. The focus is on schemes that utilize the additional spatial information offered by multiple sampling of the wave-field in multi-antenna systems to aid channel prediction. The research has led to the development of several algorithms which can be used for long range extrapolation of time-varying
\end{abstract}


channels. Based on spatial channel modeling approaches, simple and efficient methods for the extrapolation of narrowband MIMO channels are proposed. Various extensions were also developed. These include methods for wideband channels, transmission using polarized antenna arrays, and mobile-to-mobile systems.

Performance bounds on the estimation and prediction error are vital when evaluating channel estimation and prediction schemes. For this purpose, analytical expressions for bound on the estimation and prediction of polarized and non-polarized MIMO channels are derived. Using the vector formulation of the Cramer Rao bound for function of parameters, readily interpretable closed-form expressions for the prediction error bounds were found for cases with Uniform Linear Array (ULA) and Uniform Planar Array (UPA). The derived performance bounds are very simple and so provide insight into system design.

The performance of the proposed algorithms was evaluated using standardized channel models. The effects of the temporal variation of multipath parameters on prediction is studied and methods for jointly tracking the channel parameters are developed. The algorithms presented can be utilized to enhance the performance of limited feedback and adaptive MIMO transmission schemes. 


\section{Acknowledgments}

T have invested a great deal of time and effort into this research. However, it 1 would not have been possible without the support and contributions from many people, in their different ways. I would like to take this opportunity to express my sincere gratitude to them.

I thank the Almighty Allah ('God') for the wisdom and perseverance that He gave to me throughout the period of writing this thesis, and, indeed, for his guidance and protection throughout my life.

My very sincere "thank you" to my wonderful supervisors: Dr. Pawel Dmochowski and Assoc Prof. Paul Teal. First, for providing me the opportunity to pursue a $\mathrm{PhD}$ at Victoria University of Wellington and for being there for me all through the hard times. Without your useful feedback, positive criticisms and guidance, this piece of work would not have been possible. I would also like to thank my doctoral thesis examiners - Prof. Rodney Vaughan, Adjunct Prof. Mansoor Shafi and Assoc. Prof. Philippa Martin for taking the time to thoroughly examine the thesis and most importantly for the useful comments.

Sincere appreciation also goes to Prof. Bastiaan Kleijn, Dr. Christopher Hollitt and other members of the Communications and Signal Processing (CaSP) research group. It has been a wonderful experience working with you all. To Prof. Mansoor Shafi, thank you, for taking the time to examine my PhD proposal and providing useful feedback and of course, for the fatherly role you have played in my life all these years.

Many thanks to Harsh Tataria, Jaward Mirza, Praveen Chopala, Lekan 
Balogun and other colleagues and friends, who have in one way or the other made our stay in Wellington a wonderful experience. I cannot imagine how life would have been without people like you.

To my parents: Mr Rasheed Adeogun and Mrs Memunat Adeogun, I say a very big thank you, for your decades of support, prayers and guidance. I am highly grateful to my adorable wife, Ashiat Adeogun for her unflinching love, support and encouragement throughout the course of my research and for taking care of the family while I study. To my beautiful daughters, Atinuke and Omotola, thank you for the understanding and patience with daddy all through the hard times. To the PhD baby (Abdullahi Adeogun AbdulRahman Jr.), 'welcome to our world'.

Let me also use this opportunity to thank my wonderful sister; Alhaja Rafiat Alawiye (Adeogun) and her husband for their support and prayers. I pray that the Almighty God will continue to bless you abundantly. To my brothers and sisters, thank you for being there always.

I am also greatful to the Director General: Prof. Seidu Oneilo Mohammed and my superior officers at the National Space Research and Development Agency (NASRDA): Dr. Olufemi Agboola, Prof. Babatunde Rabiu, Dr Halizu Mai-Ungwa, and Engr. Sikiru Aiyeola for their support, advice and most importantly, for believing in my ability to pursue a $\mathrm{PhD}$. I am indeed blessed to have worked with wonderful people like you. To my colleagues and friends, who contributed in one way or the other to make my departure for the PhD a smooth one, I say a very big thank you.

My sincere appreciation also goes to the following people who have contributed in one way or the other to make the journey thus far a success: Hon. Isiaq Abiodun Akinlade, Eniola Joseph, Ann Okai, Kabeer Onifade, Abdullahi Abdulmalik and the numerous others whose names are not listed here, not neccessarily because they are not important, but for limitation of space. 


\section{Acronyms}

3G Third Generation of mobile telecommunications technology.

3GPP 3rd Generation Partnership Project.

4G Fourth Generation of mobile telecommunications technology.

ACF Auto-Correlation Function.

AIC Akaike Information Criterion.

AOA Angle of Arrival.

AOD Angle of Departure.

AR Autoregressive Model.

BD Block Diagonalization.

BGRW Bounded Gaussian Random Walk.

BS Base Station.

CBM Cluster Based Model.

CCF Cross-Correlation Function.

CDF Cumulative Distribution Function. 
COST European Cooperation in the field of Scientific and Technical Research.

CRB Cramer Rao bound.

CSI Channel State Information.

CSIT Channel State Information at the Transmitter.

DFT Discrete Fourier Transform.

DOA Direction of Arrival.

DPSS Discrete Prolate Spheroidal Wave Sequences.

EKF Extended Kalman Filter.

ESPRIT Estimation of Signal Parameters via Rotational Invariance Techniques.

EVD Eigenvalue Decompostion.

FCF Frequency Correlation Function.

FDD Frequency Division Duplex.

FIM Fisher information matrix.

GRW Gaussian random walk.

GSCM Geometry based stochastic channel model.

KF Kalman Filter.

LAM Linear advancement model.

LOS Line-of-Sight. 
LS Least Square.

LTE Long Term Evolution.

M-to-M Mobile-to-mobile.

MDL Minimum Description Length.

MEMCHAP Multidimensional ESPRIT based MIMO CHAnnel Predictor.

MEVD Mean Eigenvalue Decomposition.

MIMO Multiple-input multiple-output.

MISO Multiple-input single-output.

MMDL Minimum Mean Square Error Minimum Description Length.

MMSE Minimum Mean Square Error.

MP Matrix Pencil.

MS Mobile Station.

MSE Mean Square Error.

MSEB Mean squared error bound.

MSS Matrix Spatial Signature.

MSSM Matrix Spatial Signature Model.

MUSIC Multiple Signal Classification.

NLOS Non-Line-of-Sight.

NMSE Normalized Mean Squared Error.

NSE Normalized Squared Error. 
OFDM Orthogonal Frequency Division Multiplexing.

PAST Projection Approximation Subspace Tracking.

PDF Probability Density Function.

PDP Power Delay Profile.

PES Power Elevation Spectrum.

PF Particle Filter.

PRC Parametric Radio Channel.

PSWF Prolate Spheroidal Wave Function.

RMSE root mean squared error.

RSSM Receive Spatial Signature Model.

RZF Regularized Zero-Forcing.

SCF Spatial Correlation Function.

SCM Spatial Channel Model.

SIMO Single-input multiple-output.

SINR Signal-to-Interference-plus-Noise Ratio.

SISO Single-Input Single-Ouput.

SLNR Signal-to-Leakage Noise Ratio.

SNR Signal-to-Noise Ratio.

SOS Sum of Sinusoids.

STCF Spatio-Temporal Correlation Function. 
SVD Singular Value Decomposition.

TCF Temporal Correlation Function.

TDD Time Division Duplex.

TSS Transmit Spatial Signature.

TSSM Transmit Spatial Signature Model.

UCA Uniform Circular Array.

ULA Uniform Linear Array.

UPA Uniform Planar Array.

WIMAX Worldwide Interoperability for Microwave Access.

WIMEMCHAP Wideband-Multidimensional ESPRIT based MIMO CHAnnel Predictor.

WINNER Wireless World Initiative New Radio.

XPR Cross-Polarization Ratio.

ZF Zero-Forcing. 


\section{List of Symbols}

$\alpha_{p}$ Complex amplitude of the $p$ th path.

$\theta$ Azimuth Angle of Arrival (also referred to as direction of arrival).

$\vartheta$ Elevation Angle of Arrival (also referred to as direction of arrival).

$\phi$ Azimuth Angle of Departure (also referred to as direction of departure).

a bold lower case letters denote vectors.

A, $\mathcal{A}$ bold upper case and caligraphic letters denote matrices.

$\mathcal{C N}\left(\mu, \sigma^{2}\right)$ the complex Gaussian random variable with mean $\mu$ and variance $\sigma^{2}$.

$(\cdot)^{*}$ the complex conjugate.

$\mathbf{R}_{\mathrm{hh}}$ covariance/correlation matrix of observation $\mathbf{h}$.

$\tau_{p}$ delay of the $p$ th path.

$\omega_{p}$ radian Doppler frequency of the $p$ th path.

$\mathcal{I}$ Fisher information matrix.

$(\cdot)^{H}$ the Hermitian conjugate transpose.

$\mathbf{0}_{N \times M}$ an $N \times M$ matrix of zeros. 
$\mathbf{1}_{N \times M}$ an $N \times M$ matrix of ones.

$\mathbf{I}_{N}$ an $N \times N$ identity matrix.

$\mathbf{A}^{-1}$ matrix inverse.

$\mathbf{a}_{i}$ the $i$ th column of $\mathbf{A}$.

$[\mathbf{A}]_{i j}$ the $(i, j)$ th element of $\mathbf{A}$.

$\mathbf{A}^{\dagger}$ the Moore-Penrose pseudoinverse of $\mathbf{A}$.

$\eta_{p}$ normalized delay of the $p$ th path.

$\nu_{p}$ normalized Doppler frequency of the $p$ th path.

$\Theta$ a vector containing all channel parameters.

$P$ Number of propagation paths (or clusters).

$N_{\text {pol }}$ Number of polarization dimensions.

$Q$ Number of sub-paths (or rays).

$M$ Number of receive antenna elements.

J selection matrix.

$\boldsymbol{\theta}$ a vector containing parameters $\left\{\theta_{p}\right\}_{p=1}^{P}$.

$(\cdot)^{T}$ matrix/vector transpose.

tr the trace of a matrix.

$N$ Number of transmit antenna elements.

$\operatorname{vec}(\mathbf{A})$ the vectorization operation. vec stacks the columns of $\mathbf{A}$ to produce a vector.. 


\section{Contents}

Acronyms

List of Symbols $\quad$ xiii

I Background 1

1 Introduction 3

1.1 Introduction . . . . . . . . . . . . . . . . 4

1.2 MIMO Channel Prediction: A literature Survey . . . . . . . . 6

1.3 Channel Prediction Methods . . . . . . . . . . . . . . . . . 9

1.3.1 Parametric Radio Channel Model . . . . . . . . . . . . 10

1.3.2 Autoregressive Model Based Prediction . . . . . . . . . 11

1.3.3 Bandlimited Basis Expansion . . . . . . . . . . . . 12

1.4 List of Publications . . . . . . . . . . . . . . . . . . . . . . . . 13

1.5 Contributions . . . . . . . . . . . . . . . . . 14

1.6 Structure of the Thesis . . . . . . . . . . . . . . 17

1.7 General Comments . . . . . . . . . . . . . . . . 18

2 MIMO Propagation Channel 19

2.1 Radio Propagation on Multipaths . . . . . . . . . . . . . . 20

2.1.1 Multipath Transmission . . . . . . . . . . . . 21

2.1.2 Doppler Dispersion . . . . . . . . . . . . . . . 21

2.1.3 Delay Dispersion . . . . . . . . . . . . . . 22 
2.2 Basics of MIMO Channel Modeling . . . . . . . . . . . . . 23

2.2.1 MIMO System Model . . . . . . . . . . . . . . . . 24

2.2.2 Double Directional Channel Model . . . . . . . . . . 25

2.3 Mobile MiMO Channel Model . . . . . . . . . . . . . . . 25

2.3.1 Analytical Models . . . . . . . . . . . . . . 26

2.3.2 Physical Models . . . . . . . . . . . . . . . 27

2.3.3 Standardized MIMO Models . . . . . . . . . . . . . 28

2.4 WINNER II SCM Model . . . . . . . . . . . . . . . . . 29

2.4.1 Channel Model . . . . . . . . . . . . . . 30

2.4.2 Correlation Properties . . . . . . . . . . . 31

2.4.3 Details of Channel Model Implementations . . . . . . . 33

2.5 Three Dimensional MIMO Model . . . . . . . . . . . . . . 36

2.6 Summary . . . . . . . . . . . . . . . . 37

3 Parameter Estimation $\quad 39$

3.1 Data Model and Problem Formulation . . . . . . . . . . . . . 40

3.2 Subspace Based Methods . . . . . . . . . . . . . . . . . . . . 42

3.2 .1 MUSIC . . . . . . . . . . . . . . . . . 42

3.2.2 Root MUSIC . . . . . . . . . . . . . . . . . 42

3.2.3 ESPRIT . . . . . . . . . . . . . . 45

3.2.4 Unitary ESPRIT . . . . . . . . . . . . . . . 47

3.2.5 Matrix Pencil . . . . . . . . . . . . . . . 49

3.3 Adaptive Subspace Methods . . . . . . . . . . . . . . . . . 52

3.3.1 Projection Approximation Subspace Tracking (PAST) . 53

3.3.2 Projection Approximation Subspace Tracking with Deflation (PASTD) . . . . . . . . . . . . 54

3.4 Subspace Dimension Estimation . . . . . . . . . . . . . . 55

3.4.1 Minimum Description Length (MDL) . . . . . . . 56

3.4.2 Akaike Information Criterion (AIC) . . . . . . . 56

3.4.3 Minimum Mean Square Error - MDL . . . . . . . 56

3.5 Parameter Estimation for Linear Models . . . . . . . . . . 58 
3.5.1 LS Estimation . . . . . . . . . . . . . . . . 58

3.5.2 MMSE Estimation . . . . . . . . . . . . . . . 59

3.6 Cramer Rao Bound . . . . . . . . . . . . . . . . . . . 59

3.7 Summary . . . . . . . . . . . . . . . . 60

\section{Original Contributions $\quad 61$}

4 Narrowband MIMO Prediction 63

4.1 Prediction Models and Parametrization . . . . . . . . . . . . 63

4.1.1 DOA/DOD Model . . . . . . . . . . . . . . . . 66

4.1.2 Transmit Spatial Signature Model (TSSM) . . . . . . . 66

4.1.3 Receive Spatial Signature Model (RSSM) . . . . . . . . 66

4.1.4 Matrix Spatial Signature Model (MSSM) . . . . . . . 67

4.2 Data Transformation . . . . . . . . . . . . . . . . 68

4.2.1 DOA/DOD Transformation . . . . . . . . . . 68

4.2.2 TSSM Transformation . . . . . . . . . . . . . 72

4.2 .3 RSSM Transformation . . . . . . . . . . . . . 73

4.2.4 MSSM Transformation .............. 75

4.3 Translational Invariance in Multidimensional Vandermonde Matrices . . . . . . . . . . . . . . . . . . 76

4.4 Prediction Schemes . . . . . . . . . . . . . . . . . . . . . . . . 79

4.4.1 MSSM - MEMCHAP . . . . . . . . . . . . . 81

4.4.2 RSSM - MEMCHAP . . . . . . . . . . . . 85

4.4.3 TSSM - MEMCHAP . . . . . . . . . . . . . . 89

4.4.4 DOD $/ \mathrm{DOA}$ - MEMCHAP . . . . . . . . . . 91

4.5 Application to 3D Propagation Scenarios . . . . . . . . . . . 94

4.5.1 Channel Model . . . . . . . . . . . . . . . . . . 96

4.5.2 3D Prediction Based on DOD/DOA-MEMCHAP . . . 98

4.6 Simulation and Results . . . . . . . . . . . . . . . . . . . . . . 104

4.6.1 Performance Comparison . . . . . . . . . . . . 105

4.6.2 Complexity Analysis . . . . . . . . . . . . . . . . . 107 
4.7 Summary . . . . . . . . . . . . . . . . . . . . . . . 108

5 Wideband MIMO Prediction 113

5.1 Channel Models . . . . . . . . . . . . . . . . . . . . . . . . . . 114

5.1 .1 DOD $/$ DOA Model . . . . . . . . . . . 116

5.1.2 Transmit Spatial Signature Model (TSSM) . . . . . . . 116

5.1.3 Matrix Spatial Signature Model (MSSM) . . . . . . 116

5.2 Data Transformation . . . . . . . . . . . . . . . . . 117

5.2.1 DOD/DOA Transformation . . . . . . . . . 117

5.2.2 TSS Transformation . . . . . . . . . . . . . 119

5.2 .3 MSS Transformation . . . . . . . . . . . . . 120

5.3 Non-Cluster Based Prediction Methods . . . . . . . . . . . . . 121

5.3.1 DOD/DOA-WIMEMCHAP . . . . . . . . . . . 122

5.3 .2 TSSM-WIMEMCHAP . . . . . . . . . . . 126

5.3 .3 MSSM-WIMEMCHAP . . . . . . . . . . . . . 128

5.4 Cluster Based Prediction Method . . . . . . . . . . . . . . . . 129

5.4.1 Cluster Parameter Estimation . . . . . . . . . . . . 130

5.4.2 Joint Angle and Doppler Estimation . . . . . . . . . 133

5.4.3 Channel Prediction . . . . . . . . . . . . 135

5.5 Performance Evaluation . . . . . . . . . . . . . 136

5.5.1 Performance Metrics . . . . . . . . . . . . . . 136

5.5.2 Simulation Parameters . . . . . . . . . . . . . 137

5.5.3 Parameter Estimation Performance . . . . . . . . . 137

5.5.4 Prediction Performance . . . . . . . . . . . . 138

5.5.5 Prediction Performance with WINNER II Model . . . . 139

5.6 Summary . . . . . . . . . . . . . . . . . . 140

6 Mobile to Mobile Channel Prediction 143

6.1 Introduction . . . . . . . . . . . . . . . . . . . . . 143

6.2 Channel Models . . . . . . . . . . . . . . . . . . . 145

6.2.1 MIMO Mobile-to-Mobile Channel Model . . . . . . . . 145

6.2.2 Parametrized Model . . . . . . . . . . . . . . . . . 147 
6.3 Parameter Estimation and CSI Prediction . . . . . . . . . . . 148

6.3.1 Mobile Velocities Estimation . . . . . . . . . . . . . 148

6.4 Numerical Simulations . . . . . . . . . . . . . . . . . . . . 149

6.4.1 Simulation Parameters . . . . . . . . . . . . . . . . 149

6.4.2 Prediction Performance Evaluation . . . . . . . . . . 150

6.4.3 Mobile Velocity Estimation Error . . . . . . . . . . . 151

6.5 Summary . . . . . . . . . . . . . . . . . . . . 152

7 Application to Polarized MIMO Channels 155

7.1 Channel Models . . . . . . . . . . . . . . . . . . . . . . . . . . 155

7.1.1 2D Polarized MIMO Spatial Channel Model . . . . . . 156

7.1.2 Parametrized Prediction Model . . . . . . . . . . . . . 157

7.1.3 2D Polarized Wideband MIMO Model . . . . . . . . . 158

7.2 Polarized Narrowband MIMO Prediction . . . . . . . . . . . . 159

7.2.1 Data Preprocessing and Covariance Matrix Estimation 159

7.2.2 Subspace Dimension Estimation . . . . . . . . . . . . . 161

7.2.3 Joint Parameter Estimation . . . . . . . . . . . . . . . 161

7.2.4 Polarimetric Weights Estimation . . . . . . . . . . 162

7.2.5 CSI Prediction . . . . . . . . . . . . . . . . . . . 164

7.3 Polarized Wideband MIMO Prediction . . . . . . . . . . . . 164

7.3.1 Data Transformation . . . . . . . . . . . . . . 165

7.3.2 Covariance Matrix Estimation . . . . . . . . . . . 165

7.3.3 ESPRIT Based Joint Parameter Estimation . . . . . . 166

7.3.4 Polarimetric Weight Estimation and CSI Prediction . . 166

7.4 Numerical Simulation . . . . . . . . . . . . . . . . . . . . . 166

7.5 Conclusion . . . . . . . . . . . . . . . . . . 168

8 Bounds on the Prediction of Mobile MIMO Channels 171

8.1 MIMO Channel Prediction Error Bound . . . . . . . . . . . . 173

8.1.1 Narrowband Channel . . . . . . . . . . . . . . . . . 173

8.1.2 Wideband Channel . . . . . . . . . . . . . . . 177

8.1.3 3D MIMO Channel Prediction Error Bound . . . . . . 180 
8.2 Polarized MIMO Channel Prediction Error Bound . . . . . . . 183

8.3 Asymptotic Error Bound . . . . . . . . . . . . . . . . . . . . 186

8.3.1 Narrowband Channels . . . . . . . . . . . . . . 186

8.3 .2 Wideband Channels . . . . . . . . . . . . . 189

8.3.3 3D Asymptotic Prediction Error Bound . . . . . . . . . 193

8.4 Numerical Simulations . . . . . . . . . . . . . . . . . . . . . . 195

8.4.1 Narrowband MIMO Channel . . . . . . . . . . . . 195

8.4.2 Wideband MIMO Channels . . . . . . . . . . 196

8.5 Summary . . . . . . . . . . . . . . . . . . . . . 197

9 Dynamic Model Based Prediction and Parameter Tracking 201

9.1 Dynamic Prediction Model . . . . . . . . . . . . . . . . . . . . 202

9.1.1 Narrowband MIMO Channel . . . . . . . . . . . . . . . 202

9.1.2 Wideband MIMO Channel . . . . . . . . . . . . 203

9.1.3 Modeling Temporal Variation of Channel Parameters . 203

9.2 Adaptive MIMO Prediction . . . . . . . . . . . . . . . . 204

9.2.1 Effects of Parameter variation on CSI Prediction . . . . 205

9.2.2 Adaptive Multidimensional ESPRIT based MIMO CHAnnel Predictor (MEMCHAP) . . . . . . . . . . 206

9.3 Simulation and Results . . . . . . . . . . . . . . . . . . . . 214

9.4 Summary . . . . . . . . . . . . . . . . . . . . . 215

10 Conclusions and Future Research $\quad 217$

10.1 Summary of Contributions . . . . . . . . . . . . . 217

10.2 Conclusions . . . . . . . . . . . . . . . . . . 220

10.3 Future Research . . . . . . . . . . . . . . . . . . . . 221

A Contributions to Bandlimited Signal Extrapolation $\quad 225$

A.1 Introduction . . . . . . . . . . . . . . . . . 225

A.2 Bandlimited Signal Extrapolation (BSE) . . . . . . . . 226

A.2.1 Iterative Method . . . . . . . . . . . . . 226

A.2.2 Extrapolation Matrix Method (EME) . . . . . . . 227 
A.2.3 Minimum Norm Least Square Extrapolation . . . . . . 228

A.3 Prolate Spheroidal Wave Function . . . . . . . . . . . . . . . . 228

A.3.1 Properties . . . . . . . . . . . . . . . . . . . . . . . . 229

A.3.2 Signal Extrapolation Using PSWF . . . . . . . . . . 230

A.3.3 2D Prolate Spheroidal Wave Function . . . . . . . . . . 231

A.4 Application to Wireless Channel Prediction . . . . . . . . . . 234

A.4.1 Narrowband Channel . . . . . . . . . . . . . . . 235

A.4.2 Wideband Channel . . . . . . . . . . . . 237

A.5 Simulation and Results . . . . . . . . . . . . . . . . . 240

A.6 Summary . . . . . . . . . . . . . . . . . . . . . . . 241

B Proof of Performance Bound Expressions 243

B.1 Derivatives for the FIM in Narrowband Error Bound . . . . . 243

B.1.1 Derivative with Respect to $\mathfrak{R}(\boldsymbol{\alpha})$. . . . . . . . . . . . 244

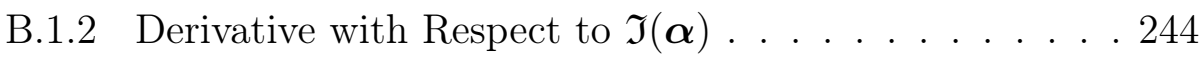

B.1.3 Derivative with Respect to $\boldsymbol{\mu}^{\mathrm{r}} \ldots \ldots . . . . . .245$

B.1.4 Derivative with Respect to $\boldsymbol{\mu}^{\mathrm{t}} \ldots \ldots . \ldots 246$

B.1.5 Derivative with Respect to $\nu$. . . . . . . . . . 246

B.2 Evaluation of FIM and Error Bound . . . . . . . . . . . . . 246

B.3 Derivatives for the FIM for Wideband Error Bound . . . . . . 247

B.3.1 Derivative with Respect $\sigma^{2} \ldots \ldots . . . . . . .247$

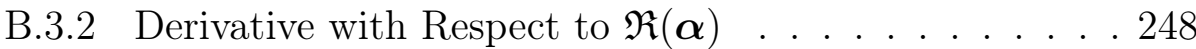

B.3.3 Derivative with Respect to $\mathfrak{I}(\boldsymbol{\alpha}) \ldots . . . .248$

B.3.4 Derivative with Respect to $\boldsymbol{\mu}^{\mathrm{r}} \ldots \ldots . . \ldots . .249$

B.3.5 Derivative with Respect to $\boldsymbol{\mu}^{\mathrm{t}} \ldots \ldots . \ldots . .249$

B.3.6 Derivative with Respect to $\nu$. . . . . . . . . . . . 250

B.3.7 Derivative with Respect to $\boldsymbol{\eta}$. . . . . . . . . . . . . 250

B.4 Evaluation of FIM and Error Bound . . . . . . . . . . . . . 250

B.5 Equivalence of Wideband Error Bound with and without Noise Variance in Parameter set . . . . . . . . . . . . . . . . . 251

B.6 Simplifications for Narrowband Asymptotic FIM . . . . . . . . 252 
B.7 Simplifications for Wideband Asymptotic FIM . . . . . . . . . 254

C System Level Performance Evaluation $\quad 257$

C.1 System Model . . . . . . . . . . . . . . . . . . . . . . . 257

C.2 Performance of Transmit Beamforming with Predicted CSIT . 260 
Part I

Background 



\section{1 \\ Introduction}

$A_{\text {important for current and future wireless communication systems where }}^{\text {CCURATE knowledge of time-varying wireless channel characteristics is }}$ the channel power may vary by several orders of magnitude over a very short spatial distance traveled by the mobile terminal. For instance, it was shown in [148] that CSI is vital to the realization of the full potential of using Multipleinput multiple-output (MIMO) techniques in wireless systems. The transmitter also requires CSI for link adaptation (e.g., modulation scheme adaptation and power allocation, scheduling, pre-equalization, adaptive transmit antenna selection and precoding $[37,40,203,215])$. Erroneous CSI often results in wasted power, high bit/symbol error rates and non-optimal throughput. In order to efficiently utilize these adaptive transmission methods, knowledge about the current as well as future channel condition is neccessary. Radio channel prediction is therefore an important technique for the realization of the potential of these transmission schemes.

This chapter presents an introduction to the MIMO wireless channel prediction problem considered in this thesis. We begin with a brief introduction 
and motivation in Section 1.1 followed by a survey of MIMO prediction literature in Section 1.2. Section 1.3 presents an overview of methods for radio channel prediction. We then present a summary of the author's contributions in Section 1.5. A list of the author's publications is presented in Section 1.4. Finally, we present an outline of the thesis structure in Section 1.6.

\subsection{Introduction}

Wireless mobile communication has witnessed a tremendous growth in the number of users, data rate requirements and coverage over the last several years. As the demand for data rate and system throughput increases, researchers and system designers need to develop efficient methods to meet the demand at reasonable complexity and cost. A common approach is to increase the bandwidth and improve the system's bandwidth utilization giving rise to a wideband frequency selective channel [120,124]. Multipath propagation, however, limits the gains that can be obtained from increased bandwidth. The need to utilize the spatial dimension of the propagation environment has resulted in systems deploying multiple antennas at both the transmitter and receiver, providing multiplexing and diversity gains. These are generally referred to as MIMO systems and have been shown to theoretically exhibit a linear relationship between channel capacity and number of antennas [26,62].

The combination of MIMO communication with Orthogonal Frequency Division Multiplexing (OFDM) [114] digital modulation is a technique for achieving high spectral efficiency and high data-rate transmission over mobile frequency selective channels [163]. It is being deployed in current and future wireless standards such as 3GPP LTE and LTE Advanced [54], IEEE 802.16e (WiMAX) [5, 58] and B3G. Recent capacity achieving MIMO-OFDM based transmission schemes such as adaptive MIMO precoding [207], adaptive coding and modulation [149], adaptive multiuser resource allocation and scheduling $[103,107]$ and various forms of codebook and non-codebook based 
limited feedback MIMO schemes [41, 121,216] can achieve high QoS and throughput for mobile wireless systems. An illustration of a feedback based linear precoded MIMO system is shown in Fig. 1.1, where the receiver estimates the CSI, quantizes it and sends the quantized channel back to the transmitter. These schemes alter the modulation scheme, subcarrier power allocation, eigenmode, and/or selected antenna(s) based on the CSI and as such require both the transmitter and receiver to have accurate knowledge of the channel state information. In TDD systems, channel reciprocity is used to obtain CSI at the receiver. In FDD systems, however, CSI is estimated at the receiver and some quantized form of the CSI is fed back to the transmitter via a low rate feedback link. In practical MIMO systems, feedback delay is inevitable due to delays in estimation, processing and feedback. The CSI may become outdated before its actual usage at the transmitter, resulting in high performance degradation especially in high mobility environments. The effects of using imperfect CSI in adaptive and limited feedback MIMO systems have been studied extensively in [61, 139, 142,151] In [191], the effects of channel aging on massive MIMO systems was studied. The authors showed that outdated CSI degrades the performance of massive MIMO systems. Prediction of the CSI into the future has therefore, been recognized has an effective technique of mitigating the performance degradation due to feedback delays $[57,72,156]$.

Although several studies have been reported on the prediction of narrowband [125, 140, 143, 187, 193, 227] and frequency selective MIMO channels $[71,99,176,204]$, the majority have been based on classical Single-Input Single-Ouput (SISO) methods which treat the MIMO channel as a number of parallel links. These approaches are sufficient when there is no correlation between antennas in MIMO systems since the knowledge about an independent signal cannot be used to improve the extrapolation of a signal. However, as a result of spacing constraints in practical MIMO systems, spatial correlation is an inevitable phenomenon [70,88, 177]. Moreover, the performance of these methods are bounded by those of the SISO schemes upon which 
they are based. Analytical and simulation results on SISO prediction have proven that with dense scattering, SISO channels can only be predicted over a very short distance. In the order of tenths of a wavelength depending on the environment and propagation scenarios. The bound on SISO channel prediction error in [190] indicates that channel prediction schemes require CSI over several wavelengths in order to accurately predict the channel and that prediction beyond a wavelength is not realistic, particularly in practical cases where the stationarity assumption does not hold long enough relative to the length of observation.

The Cramer Rao bound (CRB) on the prediction error of MIMO channels [189] and MIMO-OFDM channels [108-110] indicate that better prediction can be obtained by utilizing the spatial parameters of the MIMO channel. The CRB results show that reliable prediction of at least several wavelengths is achievable in MIMO channels. For instance, with a measurement segment length of $10 \lambda$, the prediction length (defined as the maximum prediction horizon for which the average normalized prediction error is less than 0.05) obtained from the CRB increases from about $0.25 \lambda$ for a SISO channel to about $10 \lambda$ and $30 \lambda$ for $2 \times 2$ and $3 \times 3$ MIMO channels, respectively. The development of algorithms which utilize the spatial structure of the MIMO channel to aid prediction is however still an open problem despite the huge gain that is expected.

\subsection{MIMO Channel Prediction: A literature Survey}

The problem of channel prediction for flat-fading SISO channels has been studied extensively in the past. In $[45,46,56,57,60,72,76,80,91,93,142$, 151], the narrowband SISO channel is modeled as an autoregressive (AR) process of a particular order and a linear predictor that minimizes the mean squared error (MSE) is used to predict future channel states using past esti- 


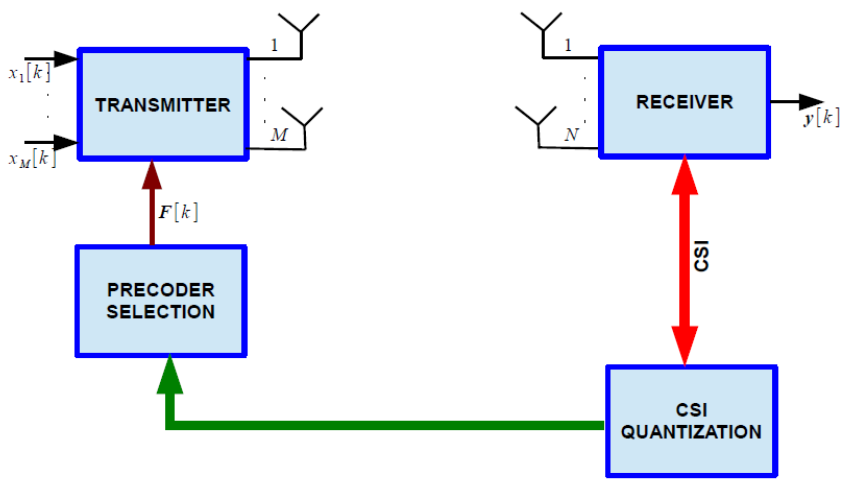

Figure 1.1: Block diagram of a $N \times M$ limited feedback linear precoded MIMO system.

mates. These schemes consider the time-varying channel as a stochastic wide sense stationary (WSS) process and use the knowledge of the temporal autocorrelation function for prediction without explicitly modeling the physical scattering phenomenon causing the fading. Since the temporal autocorrelation function may be time-varying in practical scenarios, adaptive filtering methods such as recursive least squares (RLS) [56, 132], QR-decomposition based RLS, least mean squares (LMS) [8, 95, 206] and Kalman filtering [175] have been proposed to track the temporal evolution of the AR parameters. The AR prediction methods have also been applied to frequency-selective SISO channels [117, 205].

Other researchers, $[24,118,195,214]$ have used the Parametric Radio Channel (PRC) approach where the fading channel is modeled as a sum of a finite number of plane waves. Parameter estimation algorithms are used to estimate the number of scattering sources and angles of arrival, and the channel is predicted using the model. A comparative study on different fading prediction methods for SISO channels using both synthetic and measured data is presented in [169].

The possibility of predicting multi-antenna channels was first investigated in [27] through an evaluation of downlink beamforming with channel predic- 
tion. It was shown that channel prediction improves the MISO smart antenna system performance, as a consequence of the wavefield structure revealed through multiple sampling.

The design of multipath prediction schemes for narrowband and wideband MIMO systems has been a focus of research in recent years. MIMO prediction schemes can be broadly classified into: codebook based precoder prediction and non-codebook based CSI prediction. The codebook based prediction schemes $[43,87]$ predict the precoder for the next transmission frame using linear prediction on the precoding vector. A method for predicting channel direction information (CDI) on the Grassmanian manifold using the concept of tangents, parallel transport and geodesic interpolation is presented in $[42,44]$. Other codebook based schemes adopt Givens rotation to transform the precoding matrix and perform prediction on the Givens parameter [68]. These schemes are limited to the prediction of the prediction and/or CDI one step ahead and the channel model is often assumed to be independent and identically distributed (i.i.d). Non-codebook based prediction schemes predict the actual CSI using either autoregressive modeling [31,32] and parametric model based SISO approaches [125, 188, 193]. In [226], an adaptive radio prediction method is proposed for frequency-selective MIMO channels. The method treats the channel between each antenna pair as independent SISO channels and exploits only temporal statistics for prediction. The MIMO-OFDM channel extrapolation schemes in [100, 101, 133] treat each subcarrier as a flat-fading SISO and again utilize only the temporal structure of the channel. These direct application of SISO methods do not fully utilize the spatial and temporal correlations present in the channel.

The potential gain in utilizing the spatial structure of a narrowband MIMO was illustrated in [189] using AR modelling for the prediction of a beamspace transformed CSI and an inverse transformation was performed on the predicted CSI. They argue that the transformation reduces the effective number of rays present in the channel which ultimately results in improved prediction. A similar approach based on ray canceling, which attempts to 
overcome an ill-conditioning problem with the beamspace transformation approach was presented in [143]. It was however, assumed that the Angle of Arrival (AOA) and Angle of Departure (AOD) of the dominant paths are known perfectly. This assumption is unrealistic for practical applications. In [204], a 2-step prediction method is presented which exploits the temporal and spatial correlations. This approach uses a time-domain SISO MMSE filter to each entry of the MIMO channel, followed by a spatial MMSE filtering which exploits the temporal correlation to refine the channel estimates. The spatio-temporal filtering approach is extended to MIMO-OFDM channels in [119].

As indicated in [189] while deriving bounds on the prediction of MIMO channels, parametric radio channel model based schemes are possible approaches for exploiting both the temporal and spatial correlation to improve channel prediction. However, no prediction method was presented and there has been no such method in the open literature to the best knowledge of the author.

\subsection{Channel Prediction Methods}

Channel prediction algorithms are fundamentally based on the principle of analyzing and forecasting the channel by utilizing knowledge of current and past CSI. In order to predict the future CSI samples using past channel estimates, a model is used to capture the dynamics of the fading channel. Given a set of channel estimates the slowly varying (often assumed quasistationary) parameters of the model are estimated, and the future CSI is then predicted using the model. An illustration of the generalized prediction concept is shown in Fig. 1.2. Existing schemes can be broadly categorized into: (a) Parametric radio channel model, (b) AR model and (c) bandlimited basis-expansion. A brief discussion of these methods is given in this section. 


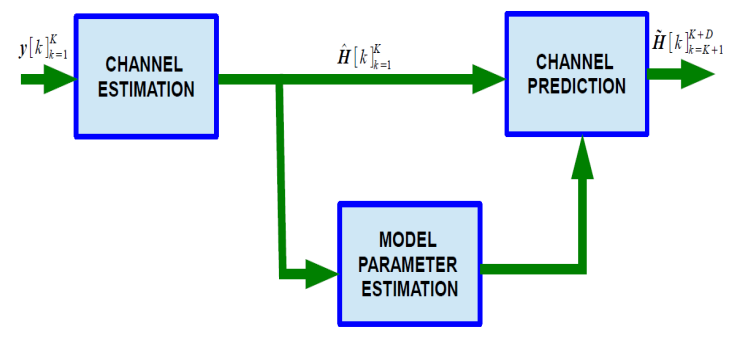

(a) Block diagram.

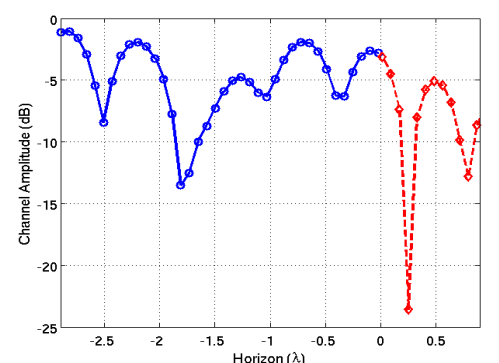

(b) Estimated and Predicted Channel

Figure 1.2: Illustration of CSI prediction concept. (a) Block diagram showing the general prediction procedure which involve channel estimation, model parameter extraction from the noisy channel estimates and CSI extrapolation using the model with the estimated parameters and (b) A plot of typical channel fading envelope showing prediction (the red line in (b)) of the CSI beyond the estimated segment (the blue line in (b)).

\subsubsection{Parametric Radio Channel Model}

Fading prediction methods based on the PRC model or Sum of Sinusoids (SOS) model $[147,150]$ are widely used in channel prediction studies. Intuitively, the PRC is an obvious approach for channel extrapolation. The PRC methods are based on the observation that the underlying multipath parameters causing fading vary much more slowly than the actual channel itself and that if these parameters are known, the channel can be extrapolated into the future. This approach models the fast fading channel as a superposition of a finite number of complex exponentials each having different amplitudes, Doppler frequencies and phases. Super-resolution spectral estimation methods are applied to estimate the Doppler frequencies associated with the complex exponentials after which, a least square fit to the known channel is utilized to estimate the amplitudes. These methods have been used extensively in the prediction of SISO channels in [24, 93, 117, 195]. 
The commonly used methods for PRC model parameter estimation are the Estimation of Signal Parameters via Rotational Invariance Techniques (ESPRIT) $[126,158,159]$ and Root-MUSIC [93]. The SISO schemes were applied to the prediction of MIMO channels in [193] and multiuser MIMO channel in [176]. Note that direct application of SISO schemes to MIMO channels is not optimal since the spatial structure of the channel is not utilized.

\subsubsection{Autoregressive Model Based Prediction}

Another class of commonly used schemes for fading channel prediction are the autoregressive (AR) models [30, 96, 184]. These schemes are based on the idea that the future behavior of the multipath channel can be predicted using a weighted linear combination of past estimates [57]. The weights (co-efficients of the model) are usually computed in order to minimize the Mean Square Error (MSE) [152]. This requires knowledge of the channel autocorrelation functions [56]. Since the autocorrelation functions are not known in practice, they are typically estimated from available noisy channels estimates via a solution of Yule Walker equations [30]. In [31, 167, 169], the Burg method was used in computing the AR coefficients. Other methods such as the covariance and modified covariance methods [184] have also been used in [169]. Although AR models have been used extensively in the study of prediction for SISO channels [57], the scheme have been shown to be highly sensitive to noise ${ }^{1}$, making even short term fading prediction challenging [64]. Moreover, the problem of error propagation ${ }^{2}$ makes this approach unattractive for long range fade prediction.

A common approach for the prediction of MIMO channel in literature is

\footnotetext{
${ }^{1}$ Noise sensitivity is often minimized by oversampling the channel, which in effect increases the Signal-to-Noise Ratio (SNR).

${ }^{2}$ Error propagation is a consequence of the fact that AR modeling of a discrete channel is usually formulated for single-step prediction using a linear extrapolation approach. Typically, prediction further steps ahead is achieved by re-using the extrapolated channel samples at previous time instants.
} 
to treat the channel as parallel SISO channels and apply a separate MMSE predictor to each of the channels [32]. This approach is not optimal as it only utilizes the temporal correlation of the individual SISO channels. Multichannel linear prediction was however used in [32] for the prediction of MIMO channels using measurement data. This approach used vector AR models to explore the spatial correlation of the MIMO channel. Although the multichannel AR approach was shown to outperform repeated application of SISO AR models, the high computational complexity which grows with increasing number of antennas makes it unattractive in practice [32].

\subsubsection{Bandlimited Basis Expansion}

Multipath prediction using the concept of bandlimited signal extrapolation is based on the idea that the signal can be decomposed into a summation of appropriate basis functions. The known segment of the signal is used to evaluate the basis functions which are then used in the model for prediction. In [202], a modal decomposition of a flat-fading SISO multipath wavefield based on the physical wave propagation equation was used to decompose the channel and extrapolate outside the known region. In [122, 223-225], the basis expansion of time-concentrated and bandlimited sequence is evaluated using the temporal autocorrelation of the channel and the extrapolated basis functions are used to predict future states of the channel. These methods are based on expansion of the channel using an appropriate basis function. In [166], the Fourier basis expansion was used to decompose the time-varying channel. It was however shown in [224] that Fourier bases suffer from the spectral leakage problem that is associated with rectangular windows. The Slepian bases (i.e., Discrete Prolate Spheroidal Wave Sequences (DPSS)) were shown to provide better representation of the time-variant channel. Although this approach was shown to yield good predictions using synthetic data, there have been very few results on real measurement, and application to the prediction of MIMO channels is still an open problem. 


\subsection{List of Publications}

The content of this thesis has been published, accepted or submitted for publication in the papers listed below.

1. R. Adeogun, P. D. Teal and P. Dmochowski, "Long Range Parametric Channel Prediction for Narrowband MIMO Systems with Joint Parameter Estimation", IEEE 7th International Conference on Signal Processing and Communication Systems (ICSPCS), Gold Coast, December, 2013.

2. _—, "Parametric Channel Prediction for Narrowband Mobile MIMO Systems Using Spatio-Temporal Correlation Analysis", in Proc. IEEE 78th Vehicular Technology Conference (VTC 2013-Fall), September 2013, pp. 1-5

3. __ , "Novel Algorithm for Prediction of Wideband Mobile MIMO Wireless Channels", in Proc. IEEE International Conference on Communications (ICC), June, 2014

4. —_, "Parametric Channel Prediction for Narrowband MIMO Systems Using Polarized Antenna Arrays", in Proc. IEEE Vehicular Technology Conference (VTC) Spring, May 2014

5. — , "Asymptotic Error Bounds on Prediction of Narrowband MIMO Wireless Channels", IEEE Signal Processing Letters, vol. 21, no. 9, 2014, pp. $1103-1107$

6. __ , Extrapolation of MIMO Mobile to Mobile Wireless Channels Using Parametric Model Based Prediction", IEEE Transactions on Vehicular Technology, vol. PP, issue 99, Nov. 2014

7. _ , "An Asymptotic Bound on Estimation and Prediction of MIMOOFDM Wireless Channels", IEEE Transactions on Vehicular Technology, 2014 (under revision) 
8. __ , "Parametric Schemes for Prediction of Wideband MIMO Wireless Channels", IEEE Transactions on Signal Processing (under revision)

\subsection{Contributions}

The contributions of the author in the field of channel prediction for mobile MIMO system is summarized as follows:

- Bandlimited channel extrapolation: Using the bandlimited property of wireless channels, we investigate the prediction of narrowband MIMO channel using bandlimited extrapolation techniques. Results obtained from our experiments showed very good prediction performance particularly for short range prediction, but this approach is not explored further in this research due to the difficulty in finding ways of utilizing the spatial information offered by multiple sampling of the channel in multiantenna systems. Motivated by the unique properties and importance of prolate spheroidal wave functions (PSWF) in bandlimited extrapolation, a solution of PSWF in 2D is derived in the earlier stage of this research. Using the properties of $2 \mathrm{D}$ discrete prolate spheroidal wave sequences (DPSS), a method is developed for the extrapolation of frequency selective channels based on the Doppler and delay bandwidths.

- Narrowband MIMO prediction using parametric modeling: A number of parametric channel predictors are proposed based on spatial modeling of the double directional channel model, where the channel is defined in terms of angles of arrival, angles of departure, and Doppler frequencies. A novel method for transforming the channel matrix is proposed to enable joint extraction of the channel parameters using subspace based methods. The predictors are named MEMCHAP (Multidimensional ESPRIT based MIMO CHAnnel Predictor), Transmit 
Spatial Signature Model (TSSM)-MEMCHAP, Receive Spatial Signature Model (RSSM) -MEMCHAP and Matrix Spatial Signature Model (MSSM)-MEMCHAP. The performance of these predictors approaches the bound with increasing SNR and/or measurement segment length. The proposed predictors are extended to MIMO propagation using polarized antenna arrays and 3D propagation, where the elevation angular parameters are also extracted in addition to the azimuth parameters.

- Wideband MIMO prediction using parametric modeling: The proposed predictors for narrowband MIMO channels are extended to wideband MIMO-OFDM channels where the delay resolutions of the system allow for the extraction of the delays. Similar to the narrowband schemes, the predictors for wideband channels are named WIdeband MEMCHAP (WIMEMCHAP), TSSM - WIMEMCHAP, and MSSMWIMEMCHAP. Motivated by the cluster based modeling approach in recent standardized channel models, cluster based approaches are also proposed. As in the narrowband case, extensions to 3D scenarios and polarized channels are also explored. Experiments using synthetic data indicate that the proposed predictors are asymptotically efficient.

- Mobile to mobile MIMO channel prediction: Mobile to mobile channels exhibit more significant variation and statistics when compared to fixed to mobile channels. Motivated by the difference in the modeling of mobile to mobile channels, we extended the prediction concept for fixed to mobile channels to M2M channels.

- Performance bounds on the prediction of MIMO channels: Motivated by the benefits of having bounds upon which the performance of MIMO multipath parameter estimation and CSI prediction algorithms can be compared, bounds on the mean square error for channel estimation and prediction in both narrowband and wideband systems are derived in this thesis. The bounds are obtained using the 
vector formulation of the deterministic Cramer- Rao bounds for functions of parameters. Similar expressions are also derived for polarized narrowband and wideband channels as well as polarized and mobile-tomobile MIMO channel. The resulting bounds are simple closed-form expressions that are readily interpretable and show the effects of various propagation and system parameters on the achievable performance in both narrowband and wideband MIMO channels.

- Velocity estimation in Mobile to Mobile channels: Based on the parametrized model for mobile to mobile channels and the estimated channel parameter, a simple scheme for extracting the velocities of the transmitter and receiver is proposed.

- Multiple invariance subspace tracking: Based on the projection alternating subspace tracking (PAST), this thesis proposes a method for jointly tracking MIMO channel parameters (AOA, AOD, Doppler frequency and/or delay).

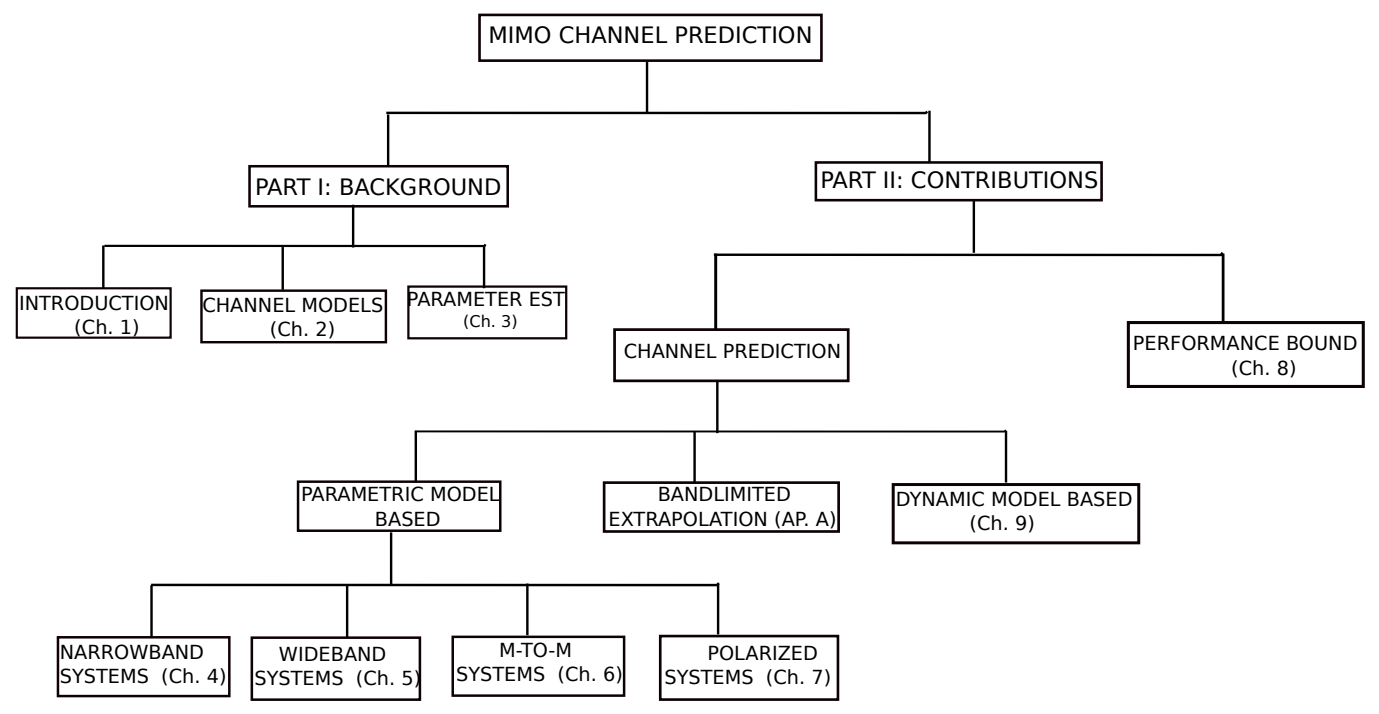

Figure 1.3: Graphical illustration of thesis outline and contributions. 


\subsection{Structure of the Thesis}

The general organization of the thesis is illustrated in Fig. 1.3. The remaining part of the thesis is structured as follows:

- MIMO propagation channel: In Chapter 2, we introduce the concept of multipath transmission and MIMO channel modeling and present standardized channel models that will be used in evaluating the proposed schemes in later part of the thesis.

- Parameter estimation: The focus of Chapter 3 is to present a review of parameter estimation methods that are considered in the development of schemes for MIMO channel prediction along with an introduction to the Cramer Rao lower bound.

- Narrowband MIMO Prediction: In chapter 4, we present the parametric algorithms developed in this thesis for narrowband MIMO channels along with extensions to 3D propagation environments.

- Wideband MIMO Prediction: Chapter 5 gives the extension of our prediction approaches to doubly selective MIMO channels. Extensions to polarized wideband MIMO channels are also discussed in this chapter.

- Mobile-to-Mobile Channel Prediction: Chapter 6 presents parametrization of mobile-to-mobile channels along with an adaptation of our prediction concept to mobile-to-mobile scenarios.

- Application to Polarized MIMO Channel: Extensions of the prediction concept in Chapters 4 and 5 to polarized narrowband and wideband channels are presented in Chapter 7

- Performance Bounds in MIMO Prediction: In chapter 8, we derive expression for the prediction error bound and asymptotic prediction error bound in narrowband and wideband MIMO channel. 
- Dynamic Modeling and Multiple Invariance Subspace Tracking: Chapter 9 presents our investigations on modeling temporal variation of propagation path parameters and develops a framework for jointly tracking the channel parameters based on multidimensional extension of subspace tracking methods.

- Conclusion and Future Research: In chapter 10, we present a summary of our research findings and identify areas for future research.

- Bandlimited signal extrapolation: Appendix A presents our investigations, methods and results on the prediction of MIMO channels using bandlimited extrapolation techniques.

\subsection{General Comments}

- The words We and Our as used throughout this thesis refer to the author in consultation with his PhD supervisors - Assoc. Prof. Paul Teal and Dr. Pawel Dmochowski.

- A Cluster refers to a group of rays with different angular parameters and a common time delay. The term cluster is used interchangeably with path in this thesis.

- The terms Ray and sub-path are used to refer to a single propagation path from the transmit array to the receive antenna. 


\section{2}

\section{MIMO Propagation Channel}

J IRELESS communication systems with multiple antennas at both the W transmit and receive end of the link have become very popular within the last two decades $[26,49,62]$. This is due to the possibility of utilizing the spatial degrees of freedom offered by multiple antenna elements to increase spectral efficiency, improve link reliability and mitigate interference. Recently, MIMO techniques have been adopted in current and future standardizations such as WIMAX [5], 3GPP LTE, IEEE 802.11n [65], and LTE-Advance [54]. In order to fully characterize the performance of MIMO systems under multipath propagation conditions, realistic physical channel models are required. The development of adequate spatial channel models for MIMO systems is currently the focus of both academic and industry research and several standardized models have been developed. These include 3GPP SCM [2], SCME [36, 137], WINNER I/II [106] and COST 273 [52].

The aim of this chapter is to present an overview of multipath propagation and modeling approaches for mobile MIMO systems along with a description and implementation of standardized models that will be used in the later 
part of this thesis. For further reading on multipath propagation and MIMO modeling, see [148, 165, 192]. Detailed description of the standardized models can be found in $[29,50,106,135]$.

\subsection{Radio Propagation on Multipaths}

In wireless mobile systems, signals are transmitted as electromagnetic waves from the transmitter to the receiver. As a result of several phenomenon such as reflection, refraction, scattering and absorption [165] inherent in the propagation environment, the signals travel through two or more paths before reaching the receiver. These paths are each characterized by different complex amplitude, delay of arrival, angle of departure (AOD), and angle of arrival (AOA). The superposition of signals from these paths at the receiver can result in either constructive or destructive interference causing the channel envelop to exhibit peaks or deep fades. The effects of these environmental impairment of the wireless transmission channel is generally termed multipath fading.

Depending on the time scale, multipath fading can be classified into: path loss, large-scale (slow) fading, and small-scale (fast) fading. Propagation path loss results from the attenuation of signal power with distance which is characterized using empirical models. Some of the commonly used path loss models are Hata and Okumura models [170]. Large scale fading result from shadowing effects of large scattering sources. This slow variation causes signal power to vary slowly around the mean and is generally described by a log-normal distribution [154]. Fast (or small scale) fading, on the other hand results from rapid variations of the phases of signal from multiple paths. The major factors contributing to this fast variation are multipath and movement of the transmitter, receiver and/or the scattering sources. These two physical phenomenon introduce frequency and temporal dispersion to the channel. In this section, we introduce the basics of multipath channel propagation along with some important characteristics. 


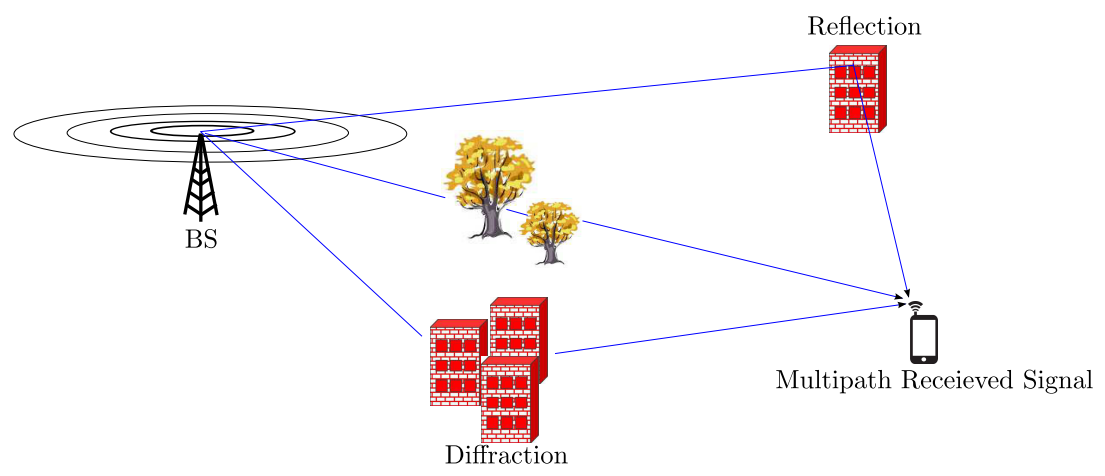

Figure 2.1: Typical mobile multipath propagation channel.

\subsubsection{Multipath Transmission}

A typical mobile propagation channel where the received signal at the mobile terminal comprises of reflected, diffracted and scattered versions of the transmitted signal is shown in Fig. 2.1.Fading in radio propagation is caused by constructive or destructive interference between two or more versions of the transmitted signal, each arriving at the receive antenna with different relative delays. These multipath signals combine vectorially to form a resultant signal which may vary in both amplitude and phase depending on the transmission bandwidth, signal power and relative propagation delays of the arriving waves. In scenarios where the mobile terminal is stationary, fading may occur due to movements in the scattering medium [154]. Multipath fading may be considered as a purely spatial phenomenon if it results from the movement of only the mobile station (i.e., with a static propagating medium). However, the spatial variations of the received signal are observed as temporal variations by the mobile station as it moves through space.

\subsubsection{Doppler Dispersion}

Doppler frequency spread is an important measure for characterizing the time varying nature of a wireless channel. Doppler shift is the frequency variation which occurs due to the relative motion between the mobile terminal 


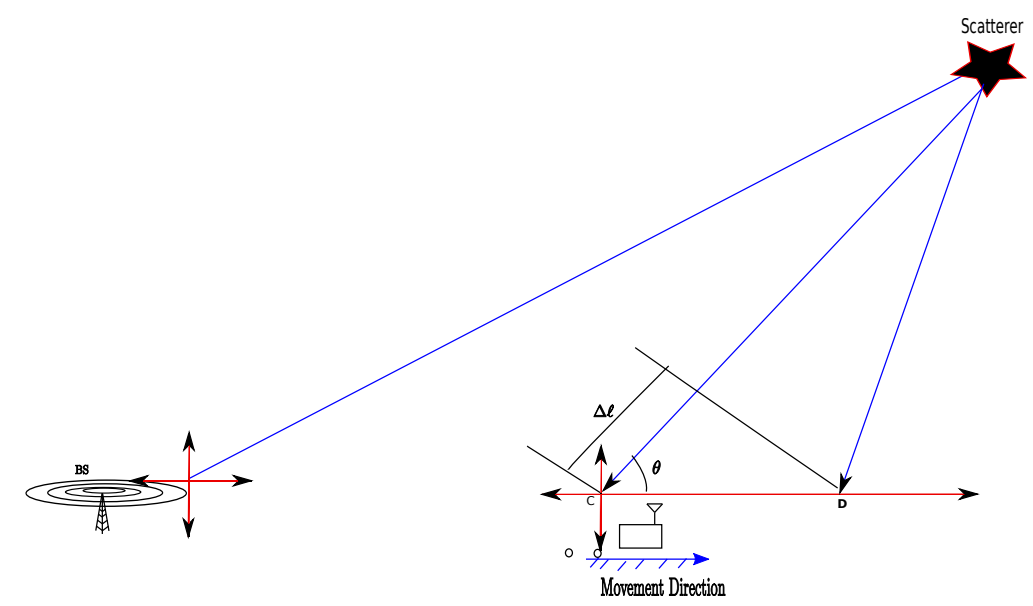

Figure 2.2: Illustration of Doppler frequency shift.

and scatterers in the propagating medium and is dependent on the mobile velocity, direction of motion and the relative direction of arrival of each signal. Figure 2.2 illustrates the concept of frequency shift due to mobility for a station traveling with constant velocity, $v$ from point $C$ to $D$ and a moving scatterer with velocity $v_{\mathrm{s}}$ in the direction of motion of the mobile station. The phase difference between the signal at point $C$ and $D$ is given by [69]

$$
\Delta \phi=k \Delta l=k v \Delta t \cos \theta
$$

where $\kappa=2 \pi / \lambda$ is the wavenumber. The Doppler frequency (in $\mathrm{rad} / \mathrm{sec}$ ) is thus [192]

$$
\omega=\frac{\Delta \phi}{\Delta t}=\frac{2 \pi v \cos \theta}{\lambda}
$$

The Doppler frequency $\omega$ can either be negative or positive depending on the direction of motion of the mobile station relative to the signal direction of arrival, causing the received signal frequency to vary between $\omega_{c}-\omega$ and $\omega_{c}+\omega_{d}$.

\subsubsection{Delay Dispersion}

The difference between the time of arrival of the scattered, reflected and/or refracted versions of a multipath signal in wireless propagation is character- 
ized by the delay/time dispersive properties of the channel. These properties are typically described by the mean excess delay $(\bar{\tau})$ and the root mean square (RMS) delay spread [192]. The mean excess delay is defined as the first moment of the power delay profile (PDP), thus

$$
\bar{\tau}=\frac{\sum_{p=1}^{P} \Lambda_{p} \tau_{p}}{\sum_{p=1}^{P} \Lambda_{p}}
$$

where $L a m b d a_{p}$ denotes the power of the pth path. The RMS delay spread is defined as the second moment of the PDP

$$
\sigma_{\tau}=\sqrt{\overline{\tau^{2}}-(\bar{\tau})^{2}}
$$

where

$$
\overline{\tau^{2}}=\frac{\sum_{p=1}^{P} \Lambda_{p} \tau_{p}^{2}}{\sum_{p=1}^{P} \Lambda_{p}}
$$

The RMS delay spread is inversely related to the coherence bandwidth (the range of frequency over which signals are equally attenuated)

$$
B_{c} \propto \frac{1}{\sigma_{\tau}}
$$

The coherence bandwidth is often used as the basis for classifying wireless channel into narrowband (frequency non-selective) or wideband (frequency selective). In narrowband channels, the transmission bandwidth is less than the coherence bandwidth of the channel. On the other hand, frequency selective channels occur when the transmission bandwidth is greater than the channel's coherence bandwidth.

\subsection{Basics of MIMO Channel Modeling}

This section presents a brief introduction of MIMO channel modeling along with the concept of double directional propagation that is commonly used in MIMO channel models. 

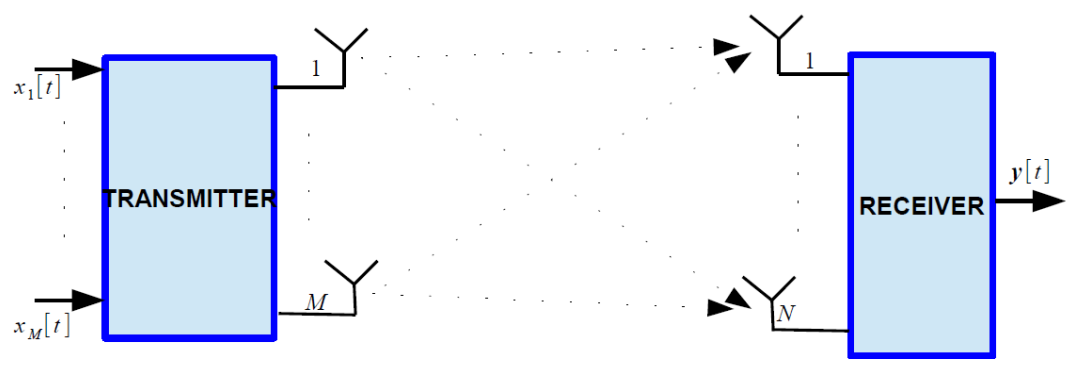

Figure 2.3: Schematic illustration of a MIMO system.

\subsubsection{MIMO System Model}

Since MIMO systems have multiple antennas at both the transmit and receive ends of the link (see Fig. 2.3), the channel between all transmit and receive antenna pairs can be described by an $N \times M$ matrix $\mathbf{H}$. For a time-varying MIMO channel, $\mathbf{H}(t, \tau)$ is defined as

$$
\mathbf{H}(t, \tau)=\left[\begin{array}{cccc}
h_{11}(t, \tau) & h_{12}(t, \tau) & \cdots & h_{1 M}(t, \tau) \\
h_{21}(t, \tau) & h_{22}(t, \tau) & \cdots & h_{2 M}(t, \tau) \\
\vdots & \vdots & \ddots & \vdots \\
h_{N 1}(t, \tau) & h_{N 2}(t, \tau) & \cdots & h_{N M}(t, \tau)
\end{array}\right]
$$

where $h_{n m}(t, \tau)$ denotes the time-variant channel impulse response between the $m$ th transmit and the $n$th receive antenna elements. Denoting the $M \times$ 1 transmit signal vector as $\mathbf{x}(t)=\left[\begin{array}{lll}x_{1}(t) \cdots & x_{M}(t)\end{array}\right]^{T}$, the received signal vector can be defined using (2.7) as

$$
\mathbf{y}(t)=\int_{\tau} \mathbf{H}(t, \tau) \mathbf{x}(t-\tau) \mathrm{d} \tau+\mathbf{w}(t)
$$

where $\mathbf{w}(t)$ models the effect of noise and interference. Note that if polarized antenna arrays are considered, each entry of (2.7) is replaced by a polarimetric submatrix, thereby increasing the total number of antennas and hence, the size of $\mathbf{H}(t, \tau)$. 


\subsubsection{Double Directional Channel Model}

The double directional impulse response of a wireless propagation channel models the overall effect of the transmit antenna array, transmission medium and receive antenna array. In the absence of polarization, the double directional impulse response between the $n$th receive and $m$ th transmit antenna is defined as $^{1}[23,69]$

$$
h_{n m}(t, \boldsymbol{\tau}, \boldsymbol{\theta}, \boldsymbol{\phi})=\sum_{p=1}^{P} \alpha_{p} \delta\left(\tau-\tau_{p}\right) \delta\left(\theta-\theta_{p}\right) \delta\left(\phi-\phi_{p}\right)
$$

where $\alpha_{p}, \tau_{p}, \theta_{p}$ and $\phi_{p}$ denote the complex amplitude, delay, angle of arrival and angle of departure associated with the $p$ th path, respectively. If doubly polarized antennas are used, $\alpha_{p}$ is replaced by a $2 \times 2$ polarimetric weight matrix

$$
\mathbf{G}_{p}(t)=\left[\begin{array}{ll}
g_{p}^{\mathrm{vv}} & g_{p}^{\mathrm{vh}} \\
g_{p}^{\mathrm{hv}} & g_{p}^{\mathrm{hh}}
\end{array}\right]
$$

where $\mathrm{v}$ and $\mathrm{h}$ denote the vertical and horizontal polarizations, respectively. As shown in (2.9), each arrival angle is associated with a corresponding departure angle in the double directional model.

\subsection{Mobile MIMO Channel Model}

The performance of any mobile wireless communication system is largely dependent on the characteristics of the propagation environment. The inherent temporal and spatial variations in mobile MIMO wireless channel constitute a major drawback to the attainment of the theoretical gains of using multiple antennas. Channel models are developed to simulate the propagation environment in the development of algorithms for wireless communication systems. There are several classifications of wireless channel models viz:

\footnotetext{
${ }^{1}$ This is based on the assumption of $2 \mathrm{D}$ propagation without account for the elevation spectrum.
} 
time-invariant versus time-variant, flat fading (narrowband) versus frequency selective (wideband), analytical versus physical, and so on.

A wireless channel is said to undergo flat fading if the channel has a constant attenuation and linear phase over a bandwidth greater than the transmission bandwidth (i.e., $B_{\mathrm{t}}<<B_{\mathrm{c}}$, where $B_{\mathrm{t}}$ and $B_{\mathrm{c}}$ are the transmission and channel coherence bandwidths, respectively) [192]. In contrast, if the bandwidth over which the phase is linear and the attenuation is constant is less than the transmission bandwidth, the channel is said to be frequency selective.

The major distinction between time-varying and time-invariant channels is that time-varying channels require characterization of the temporal dynamics resulting from movement of the mobile station and/or scatterers.

Analytical MIMO models use a simplistic approach that independently models the spatial correlation using a user defined correlation matrix and temporal correlation using the Doppler spectrum. Physical models on the other hand, are based on the underlying electromagnetic wave propagation mechanism, and while generally more accurate they require more complex computation. The focus of this section will be on comparing analytical and physical MIMO models along with a brief discussion of standardized MIMO models. Detailed review and literature survey on MIMO channel modeling can be found in [23].

\subsubsection{Analytical Models}

Analytical models use mathematical representations to characterize the impulse response of the channel without account of the actual propagation phenomenon causing the fading. Commonly used analytical models are correlation based. These models characterize the MIMO channel impulse response matrix in terms of an independent and identically distributed (i.i.d) matrix and the spatial correlation between entries of the matrix. Examples of correlative analytical models are the classical (iid) model, Kronecker model, 
and Weichselberger model [200]. In the classical model, entries of the MIMO matrix are independently and identically distributed Gaussian random variables with variance equal to the transmit power ${ }^{2}$. The Kronecker model is based on the assumption that the transmit and receive angular spectra are separable which allows us to separate the transmit and receive correlation matrices. The MIMO matrix of the Kronecker model is given by [69]

$$
\mathbf{H}_{\mathrm{kron}}=\mathbf{R}_{\mathrm{r}}^{\frac{1}{2}} \mathbf{H}_{\mathrm{idd}} \mathbf{R}_{\mathrm{t}}^{\frac{1}{2}}
$$

where $\mathbf{R}_{\mathrm{r}}=\mathbb{E}\left[\mathbf{H H}^{H}\right]$ and $\mathbf{R}_{\mathrm{t}}=\mathbb{E}\left[\mathbf{H}^{H} \mathbf{H}\right]$ are the receive and transmit correlation matrices, $\mathbf{H}_{\text {iid }}$ has i.i.d zero mean Gaussian entries and $\mathbb{E}[\cdot]$ denotes the expectation operator. When $\mathbf{R}_{\mathrm{r}}=\mathbf{R}_{\mathrm{r}}=\rho^{2} \mathbf{I}$, the Kronecker model reduces to the classical i.i.d Rayleigh channel. The Weichselberger model eliminates the unrealistic correlation matrix separability assumption in the Kronecker model [144]. This model is based on the eigendecomposition of the transmit and receive correlation matrices and the MIMO matrix is given by [200]

$$
\mathbf{H}_{\mathrm{wei}}=\mathbf{U}_{\mathrm{r}}\left(\Omega \odot \mathbf{H}_{\mathrm{iid}}\right) \mathbf{U}_{\mathrm{t}}^{T}
$$

where $\odot$ denotes element-wise (Hadamard) multiplication, $\mathbf{U}_{\mathrm{r}}$ and $\mathbf{U}_{\mathrm{t}}$ are the eigenvectors of the receive and transmit correlation matrix respectively and $\Omega$ is the power coupling matrix which gives the average energy coupling between the transmit and receive eigenvectors.

\subsubsection{Physical Models}

Physical MIMO models are based on the electromagnetic wave propagation mechanism, where the channel is modeled in terms of physical parameters such as angle of departure (AOD), angle of arrival (AOA), complex attenuation coefficients, antenna location and the carrier frequency. These models are generally based on the concept of double directional propagation

\footnotetext{
${ }^{2}$ It should be noted that all propagation effects such as shadowing and path-loss are absorbed into the variance of the channel.
} 
$[135,182]$ between the location of the transmit antenna array and the receive antenna array. Physical channel models can be classified into Geometry based stochastic channel model (GSCM), nongeometry based stochastic models and deterministic models [23]. In the GSCM, the channel is obtained by applying the electromagnetic wave propagation mechanism to specific transmit array, receive array and scatterer geometries. The antenna and scatterer geometries are selected randomly. On the other hand, non-geometry based channel models characterizes the impulse response in a completely stochastic manner. The parameters of the channel (i.e., AOA, AOD, delay, etc) are chosen based on specified probability distributions, such as Von-Misses, uniform and truncated Gaussian without the assumption of any antenna and/or scatterer geometry. In order to distinguish different propagation environments, the parameters may be estimated for different statistical distributions based on channel measurements. Deterministic channel models describe the MIMO channel impulse response in a completely deterministic manner by reproducing the actual propagation process for the specific environment. Common examples are ray tracing models [92]. Although these models are generally accurate, they are largely dependent on propagation environment.

Ray based standardized spatial channel models such as COST 259/273 [135], 3GPP SCM and WINNER II [106] are examples of physical MIMO models. These are more suitable for the development of fading prediction algorithms as they are based on realistic channel measurements and allow us to concentrate on the actual parameters causing the fading.

\subsubsection{Standardized MIMO Models}

Standardized channel models are essential for the development and evaluation of algorithms for mobile radio systems. They allow the use of typical system parameters that are defined within wireless standards in the evaluation of new algorithms. For example, the 3GPP/WINNER II SCM models are included in current and future generation MIMO standards within the LTE framework. 
Table 2.1: Summary of standardized channel models.

\begin{tabular}{lllll}
\hline Channel Model & Origin & Frequency $(\mathrm{GHz})$ & Bandwidth & No. of Scenarios \\
\hline COST 259/273 & COST & $0.45-5$ & 100 & $13 / 15$ \\
SCM & 3GPP/3GPP2 & 2 & 5 & 3 \\
SCME & WINNER & $2-6$ & 20 & 3 \\
WIN I & WINNER I & $2-6$ & 100 & 7 \\
WIN II & WINNER II & $2-6$ & 100 & 17 \\
IEEE 802.11n & IEEE 802.11 & 2,5 & 100 & 6 \\
\hline
\end{tabular}

A summary of selected standardized MIMO channel models is presented in Table 2.1. Detailed descriptions of the models can be found in $[29,50,106$, 135]. Comparative studies on the WINNER II channel models can be found in [138] and a comparison of the WINNER and COST channel models is presented in [136]. The WINNER II model is used in this thesis for evaluating the performance of the proposed algorithms and will be presented in a more detail in Section 2.4 .

\subsection{WINNER II SCM Model}

The WIN II model is latest version of channel models developed within ISTWINNER $[1,3,106]$. The model is based on the principle of geometry spatial channel modeling (GSCM), the drop concept and a generic modeling approach, where a generic structure is used to describe all scenarios. The WIN II model is an extension of previous channel models (WIN I and SCME) and is related to the COST 259 and the $3 \mathrm{GPP}-\mathrm{SCM}$ model. The parametrization of the model is based on several measurements conducted by the five partners involved in the project. WIN II models have been developed for seventeen indoor and outdoor scenarios with frequency range 2-6 GHz and bandwidth up to $100 \mathrm{MHz}$. A brief discussion of the channel impulse response and statistical properties of the model, and two scenarios considered for the simulations in this thesis is presented in this section. 


\subsubsection{Channel Model}

Figure 2.4 illustrates the modeling concept that is used within the Wireless World Initiative New Radio (WINNER) framework. The channel between each pair of transmit and receive antenna is modelled as a summation of finite number of multipath components (MPCs) referred to as clusters ${ }^{3}$. The term cluster will be used throughout the rest of this thesis to refer to a group of rays sharing a common delay. The MIMO channel matrix is defined as

$$
\mathbf{H}(t ; \tau)=\sum_{p=1}^{P} \mathbf{H}_{p}(t ; \tau) \delta\left(\tau-\tau_{p}\right)
$$

where $P$ is the number of clusters. The channel for the $p$ th cluster is composed of the transmit array response, $\mathbf{S}_{\mathrm{t}}$, the receive array response, $\mathbf{S}_{\mathrm{r}}$ and the propagation channel matrix, $\mathbf{F}_{p}$ as

$$
\mathbf{H}_{p}(t ; \tau)=\int_{\theta} \int_{\phi} \mathbf{S}_{\mathrm{r}}(\theta) \mathbf{F}_{p}(t ; \tau, \theta, \phi) \mathbf{S}_{\mathrm{t}}^{T}(\phi) \mathrm{d} \theta \mathrm{d} \phi
$$

For singly polarized propagation, the channel impulse response of the $p$ th cluster between transmit antenna $m$ and receive antenna $n$ is

$$
h_{p}^{n m}(t ; \tau)=\sum_{q=1}^{Q} \alpha_{p, q} e^{\left.j 2 \pi\left\{\lambda^{-1}\left(\overline{\boldsymbol{\theta}}_{p, q}^{T} \overline{\mathbf{r}}_{\mathrm{r}, n}\right\}\right)+\lambda^{-1}\left(\overline{\boldsymbol{\phi}}_{p, q}^{T} \overline{\mathbf{r}}_{\mathrm{t}, m}\right\}\right)+\omega_{p, q} t} \delta\left(\tau-\tau_{p, q}\right)
$$

where $\alpha_{p, q}$ is the complex attenuation of the $q$ th ray within the $p$ th cluster, $\lambda$ is the carrier wavelength and $Q$ is the number of rays within each cluster. $\overline{\boldsymbol{\phi}}_{p, q}$ and $\overline{\boldsymbol{\theta}}_{p, q}$ are the unit vectors in the direction of departure and arrival of the $q$ th ray, respectively. $\overline{\mathbf{r}}_{\mathrm{t}, m}$ and $\overline{\mathbf{r}}_{\mathrm{r}, n}$ are the position vectors of the $m$ th transmit and $n$th receive antenna elements, respectively. Finally, $\omega_{p, q}$ and $\tau_{p, q}$ denote the Doppler frequency and delay of the $p, q$ th ray, respectively. The model for propagation with doubly polarized antenna array is obtained

\footnotetext{
${ }^{3} \mathrm{~A}$ cluster is defined as a propagation path that is diffused in space, either in the angle and/or delay domains.
} 
by replacing $\alpha_{p, q}$ in $(2.15)$ with

$$
\beta_{p, q}=\left[\begin{array}{c}
f_{\mathrm{r}, n}^{\mathrm{v}}\left(\theta_{p, q}\right) \\
f_{\mathrm{r}, n}^{\mathrm{h}}\left(\theta_{p, q}\right)
\end{array}\right]^{T}\left[\begin{array}{ll}
\alpha_{p, q}^{\mathrm{vv}} & \alpha_{p, q}^{\mathrm{vh}} \\
\alpha_{p, q}^{\mathrm{hv}} & \alpha_{p, q}^{\mathrm{hh}}
\end{array}\right]\left[\begin{array}{c}
f_{\mathrm{t}, m}^{\mathrm{v}}\left(\phi_{p, q}\right) \\
f_{\mathrm{t}, m}^{\mathrm{h}}\left(\phi_{p, q}\right)
\end{array}\right]
$$

where $f_{\mathrm{r}, n}^{\mathrm{v}}$ and $f_{\mathrm{r}, n}^{\mathrm{h}}$ are the $n$th receive antenna field patterns for the vertical (v) and horizontal (h) polarizations, respectively. Similarly, $f_{\mathrm{t}, m}^{\mathrm{v}}$ and $f_{\mathrm{t}, m}^{\mathrm{h}}$ are the $m$ th transmit antenna field patterns.

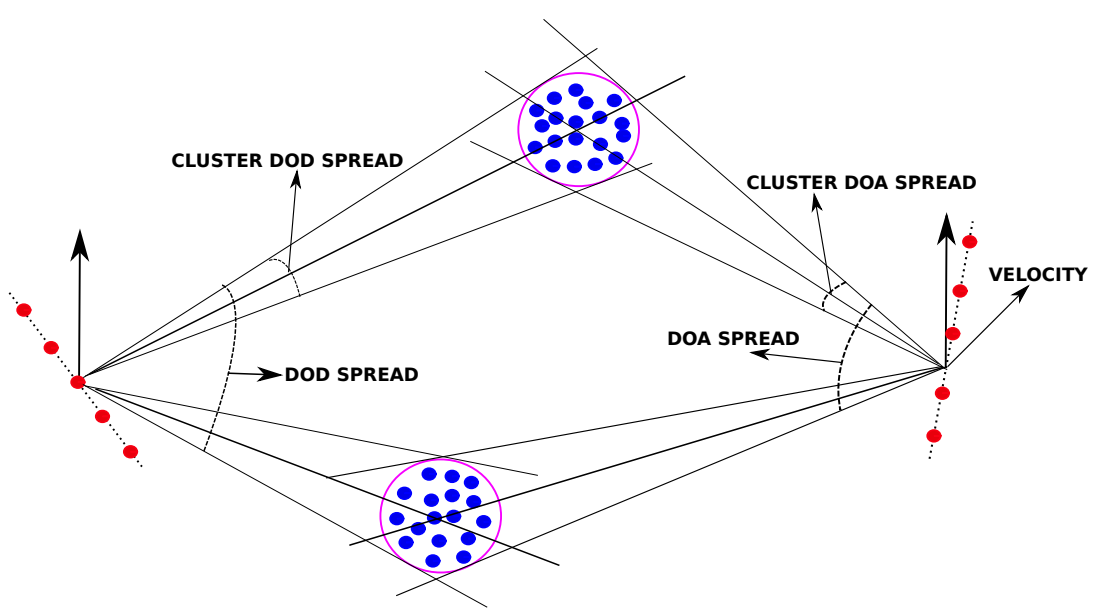

Figure 2.4: Illustration of Ray Clustering in WINNER Channel Models.

\subsubsection{Correlation Properties}

The prediction schemes developed in this thesis depend on the correlation statistics of the MIMO channel. These properties will be derived here for the WINNER channel models. Consider a uniform linear array (ULA) with element spacings, $d_{\mathrm{t}}$ and $d_{\mathrm{r}}$ at the transmitter and receiver, respectively. The channel model in (2.15) then becomes ${ }^{4}$

$$
h_{n m}(t)=\sum_{q=1}^{Q} \alpha_{p, q} e^{j 2 \pi\left\{(n-1) d_{\mathrm{r}} \sin \theta_{p, q}+(m-1) d_{\mathrm{t}} \sin \phi_{p, q}+\nu_{p, q} t\right\}}
$$

\footnotetext{
${ }^{4}$ Here, we consider a narrowband system and eliminate the dependence of the model on cluster delays.
} 


\section{Spatio-Temporal Correlation}

The complex spatio-temporal correlation function (STCF) between any two channel coefficients $h^{n_{1} m_{1}}$ and $h^{n_{2} m_{2}}$ for time interval separated by $\Delta t$ is defined as

$$
\rho_{\mathrm{st}}\left(d_{\mathrm{r}}, d_{\mathrm{t}}, \Delta_{\mathrm{t}}\right)=\mathbb{E}\left\{\frac{h_{n_{1} m_{1}}(t) h_{n_{2} m_{2}}^{H}\left(t+\Delta_{\mathrm{t}}\right)}{\sqrt{\left|h_{n_{1} m_{1}}(t)\right|^{2}\left|h_{n_{2} m_{2}}(t)\right|^{2}}}\right\}
$$

where $\mathbb{E}\{\cdot\}$ denotes statistical expectation. Substituting (2.17) into (2.18) and simplifying yields

$$
\rho_{\mathrm{st}}\left(d_{\mathrm{r}}, d_{\mathrm{t}}, \Delta_{\mathrm{t}}\right)=\sum_{p=1}^{P} \mathbb{E}\left\{e^{j k\left\{\left|\left(n_{1}-n_{2}\right)\right| d_{\mathrm{r}} \sin \theta_{p, q}+\left|\left(m_{1}-m_{2}\right)\right| d_{\mathrm{t}} \sin \phi_{p, q}+\nu_{p, q} \Delta_{\mathrm{t}}\right\}}\right\}
$$

Note that the STCF in (2.19) is a function of the AOAs, AODs and Doppler frequencies. This property will be explored later in this thesis.

\section{Temporal Correlation}

The Temporal Correlation Function (TCF), $\rho_{\mathrm{t}}$, can be obtained from (2.19) by setting $d_{\mathrm{r}}=d_{\mathrm{t}}=0$, that is

$$
\begin{aligned}
\rho_{t}\left(\Delta_{\mathrm{t}}\right) & =\left.\rho_{\mathrm{st}}\right|_{d_{\mathrm{r}}=0 ; d_{\mathrm{t}}=0} \\
& =\sum_{p=1}^{P} \mathbb{E}\left\{e^{j k\left\{\nu_{p, q} \Delta_{\mathrm{t}}\right\}}\right\}
\end{aligned}
$$

which is dependent only on the Doppler frequencies and the temporal interval.

\section{Spatial Correlation}

Similarly, the Spatial Correlation Function (SCF) is obtained from (2.19) as

$$
\begin{aligned}
\rho_{\mathrm{s}}\left(d_{\mathrm{r}}, d_{\mathrm{t}}\right) & =\left.\rho_{\mathrm{st}}\right|_{\Delta_{\mathrm{t}}=0} \\
& =\sum_{p=1}^{P} \mathbb{E}\left\{e^{j k\left\{\left|\left(n_{1}-n_{2}\right)\right| d_{\mathrm{r}} \sin \theta_{p, q}+\left|\left(m_{1}-m_{2}\right)\right| d_{\mathrm{t}} \sin \phi_{p, q}\right\}}\right\}
\end{aligned}
$$


Table 2.2: Correlation properties of spatial channel model and dependence on channel parameters.

\begin{tabular}{lllll}
\hline Property & STCF & TCF & SCF & FCF \\
\hline Parameters & AOA, AOD, Doppler & Doppler & AOA, AOD & Delay \\
\hline
\end{tabular}

The SCF as seen in (2.21) is dependent on the AOA and AOD. A summary of the correlation properties and dependence on channel parameters is given in Table 2.2.

\section{Frequency Correlation}

The Frequency Correlation Function (FCF) can be obtained from the average Power Delay Profile (PDP) using the Fourier transform [136]

$$
\rho_{\mathrm{f}}=\frac{\sum_{p=1}^{P} \Lambda_{p} e^{-j 2 \pi \tau_{p} \Delta_{\mathrm{f}}}}{\sum_{p=1}^{P} \Lambda_{p}}
$$

where $\Delta_{\mathrm{f}}$ is the frequency spacing.

\subsubsection{Details of Channel Model Implementations}

We now present two standardized channel models developed within the WINNER project [106] that will be used throughout the thesis.

\section{C2 Urban Macro-cell NLOS Channel}

The WINNER C2 urban macro-cell non-line-of-sight channel characterizes a typical urban macro-cell scenario comprising a mobile station located outdoors at street level and a fixed BS with some height above surrounding buildings. This corresponds to a propagation scenario with only non line of sight (NLOS) clusters with an uncorrelated and Rayleigh distributed fading envelope. The PDP of the C2 model is shown in Table $2.3^{5}$ and plots of the

\footnotetext{
${ }^{5}$ Note that the C2 NLOS channel provides option for using intra-cluster delay spread. In the absence of intra-cluster delay spreading, the number of paths is 20 .
} 
Table 2.3: Power delay profile of the WINNER II C2 NLOS Channel. The channel comprises of 24 clusters with corresponding delays and power

\begin{tabular}{lllllllll}
\hline Cluster & 1 & 2 & 3 & 4 & 5 & 6 & 7 & 8 \\
\hline Delay [ns] & 0 & 60 & 75 & 145 & 150 & 155 & 150 & 190 \\
Power [dB] & -6.4 & -3.4 & -2.0 & -3.0 & -5.2 & -7.0 & -1.9 & -3.4 \\
\hline Cluster & 9 & 10 & 11 & 12 & 13 & 14 & 15 & 16 \\
\hline Delay [ns] & 220 & 225 & 230 & 335 & 370 & 430 & 510 & 685 \\
Power [dB] & -3.4 & -5.6 & -7.4 & -4.6 & -7.8 & -7.8 & -9.3 & -12.0 \\
\hline Cluster & 17 & 18 & 19 & 20 & 21 & 22 & 23 & 24 \\
\hline Delay [ns] & 725 & 735 & 800 & 960 & 1020 & 1100 & 1210 & 1845 \\
Power [dB] & -8.5 & -13.2 & -11.2 & -20.8 & -14.5 & -11.7 & -17.2 & -16.7 \\
\hline
\end{tabular}

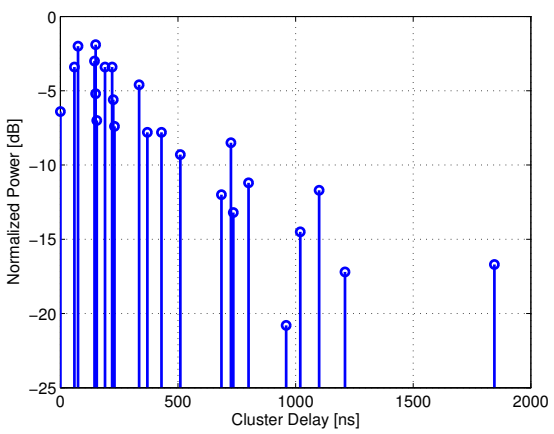

(a) PDP.

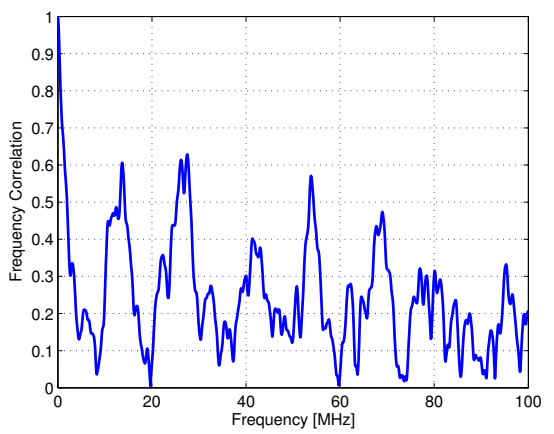

(b) Frequency Spectrum

Figure 2.5: Power delay profile and frequency correlation spectrum of the WINNER II C2 NLOS channel.

PDP along with frequency correlation are presented in Fig. 2.5. 


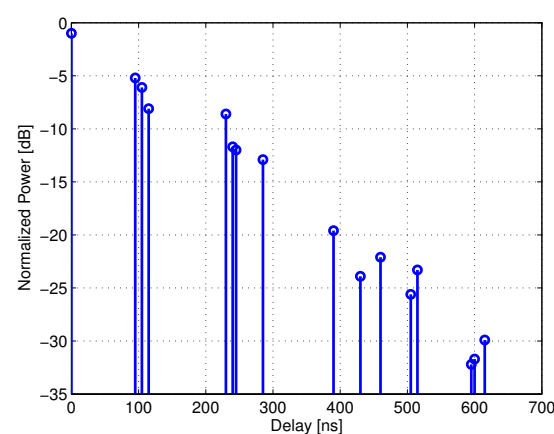

(a) PDP

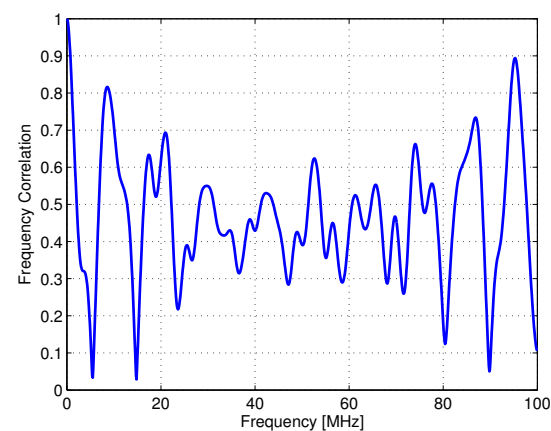

(b) Frequency Spectrum

Figure 2.6: Power delay profile and frequency correlation spectrum of the WINNER II B1 NLOS channel.

Table 2.4: Power delay profile of the WINNER II B1 NLOS Channel. The channel comprises of 16 clusters with corresponding delays and power.

\begin{tabular}{lllllllll}
\hline Cluster & 1 & 2 & 3 & 4 & 5 & 6 & 7 & 8 \\
\hline Delay [ns] & 0 & 95 & 105 & 115 & 230 & 240 & 245 & 285 \\
Power [dB] & -1.0 & -5.2 & -6.1 & -8.1 & -8.6 & -11.7 & -12.0 & -12.9 \\
\hline Cluster & 9 & 10 & 11 & 12 & 13 & 14 & 15 & 16 \\
\hline Delay [ns] & 390 & 430 & 460 & 505 & 515 & 595 & 600 & 615 \\
Power [dB] & -19.6 & -23.9 & -22.1 & -25.6 & -23.3 & -32.2 & -31.7 & -29.9 \\
\hline
\end{tabular}

\section{B1 Urban Micro Cell NLOS Channel}

The B1 NLOS channel models propagation in outdoor urban micro-cell scenarios where the height of the BS and MS antenna elements are below the top of the surrounding buildings. The model comprises of 16 clusters with different delays and power as listed in Table 2.4. The power delay profile and frequency correlations are shown in Fig. 2.6. A summary of the large and small scale parameters of the two scenarios as specified in the WINNER/3GPP channel models [3] is presented in Table 2.5. 
Table 2.5: Table of Parameters for the B1 NLOS and C2 NLOS Scenarios.

\begin{tabular}{|c|c|c|c|}
\hline Scenarios & & B1 NLOS & C2 NLOS \\
\hline \multirow{2}{*}{ Delay Spread (DS) } & $\mu$ & -7.12 & -7.12 \\
\hline & $\sigma$ & 0.12 & 0.33 \\
\hline \multirow{2}{*}{ AOD Spread (ASD) } & $\mu$ & 1.19 & 0.90 \\
\hline & $\sigma$ & 0.21 & 0.36 \\
\hline \multirow{2}{*}{ AOA Spread (ASA) } & $\mu$ & 1.55 & 1.65 \\
\hline & $\sigma$ & 0.20 & 0.30 \\
\hline Shadow Fading $(\mathrm{SF})[\mathrm{dB}]$ & $\mu$ & 4 & 8 \\
\hline Delay distribution & & Uniform $(\leq 800 n s)$ & Exponential \\
\hline \multicolumn{2}{|c|}{ AoD and AoA distribution } & \multicolumn{2}{|c|}{ Wrapped Gaussian } \\
\hline \multirow{2}{*}{$\mathrm{XPD}[\mathrm{dB}]$} & $\mu$ & 8 & 4 \\
\hline & $\sigma$ & 3 & 3 \\
\hline Number of clusters & & 16 & 14 \\
\hline \multicolumn{2}{|l|}{ Number of rays per cluster } & 20 & 20 \\
\hline Cluster ASD & & 10 & 2 \\
\hline Cluster ASA & & 22 & 10 \\
\hline
\end{tabular}

\subsection{Three Dimensional MIMO Model}

The channel models described so far are two-dimensional models and do not account for contributions from the elevation spectrum. It has however been shown [171] that the elevation spectrum cannot be neglected in MIMO 
channel modeling, particularly in indoor and in-vehicle scenarios where reflections from walls and ceiling contribute to the received signal. There have been several studies within the last few years on three dimensional (3D) modeling for MIMO channels (see $[55,171,198]$ and the references therein). We now present a generalized framework for 3D channels which will be used for $3 \mathrm{D}$ prediction in later chapters. The 3D channel impulse response as defined in the WINNER model is given as $[6,106,136]$

$$
h_{n, m}(t ; \tau)=\sum_{p=1}^{P} \sum_{q=1}^{Q} \mathbf{f}_{\mathrm{r}, n}^{T}\left(\theta_{p, q}, \vartheta_{p, q}\right) \mathbf{G}_{p, q} \mathbf{f}_{\mathrm{t}, m}\left(\phi_{p, q}, \varphi_{p, q}\right) e^{j 2 \pi \nu_{p, q} t} \delta\left(\tau-\tau_{p, q}\right)
$$

where $\mathbf{G}_{p, q}$ is the $2 \times 2$ polarimetric weight matrix in $(2.17)$ and $\mathbf{f}_{\mathrm{r}, n}\left(\theta_{p, q}, \vartheta_{p, q}\right)$ is the $3 \mathrm{D}$ complex polarimetric response of the $n$th receive antenna element defined as

$$
\mathbf{f}_{\mathrm{r}}\left(\theta_{p, q}, \vartheta_{p, q}\right)=\left[\begin{array}{l}
f_{\mathrm{v}}(\theta, \vartheta) \\
f_{\mathrm{h}}(\theta, \vartheta)
\end{array}\right] e^{j \mathbf{k}(\theta, \vartheta) \mathbf{d}}
$$

where

$$
\mathbf{d}=\left[\begin{array}{l}
d_{\mathrm{x}} \\
d_{\mathrm{y}} \\
d_{\mathrm{z}}
\end{array}\right]
$$

denotes the spatial location of the antenna element and $\kappa(\theta, \vartheta)$ is the $3 \mathrm{D}$ wave vector given by

$$
\mathbf{k}(\theta, \vartheta)=\frac{2 \pi}{\lambda}\left[\begin{array}{lll}
\sin \theta \cos \vartheta & \sin \theta \sin \vartheta & \cos \theta
\end{array}\right]
$$

\subsection{Summary}

This chapter presented a summary of multipath propagation and channel modeling for MIMO wireless systems. A description of standardized models that will be used in evaluating the performance of the predictors developed in this thesis along with the correlation properties of wireless channels were also discussed. Finally, a generalized 3D channel model was introduced to enable the development of 3D prediction algorithms in later chapters. 


\section{3}

Parameter Estimation

Es

STIMATION of propagation parameters such as Direction of Arrival (DOA) and delay are important array signal processing problems [181] in wireless communications [112,196], radar [77,113], and sonar [173, 174]. Parameter estimation methods are generally categorized as classical, subspace and maximum likelihood methods. Of these methods, the subspace based methods $[130,159,183,199]$ have been widely used in recent times due to their computational simplicity and relatively high resolution.

In this chapter, we present an overview of the parameter estimation methods that are used in the development of prediction algorithms presented in later chapters. We begin with an introduction to the data model for one dimensional estimation problems along with subspace based methods including ESPRIT [159] and Multiple Signal Classification (MUSIC) [168, 183]. Selected variants of the ESPRIT and MUSIC methods are also introduced. We then introduce subspace tracking methods and techniques for estimating the parameters of linear models. Finally, we present a review of the CRB. 


\subsection{Data Model and Problem Formulation}

Consider a ULA consisting of $N$ antenna elements. Suppose that $P(1 \leq$ $P \leq N-1)$ independent far-field narrowband signals $s_{p}(t)$ impinge on the array with arrival angles $\left\{\theta_{p}\right\}_{p=1}^{P}$. Let $\mathbf{a}\left(\theta_{p}\right)$ denote the array response vector associated with the $p$ th source. The noisy observation data $\hat{\mathbf{y}}(t)$ is

$$
\begin{aligned}
\hat{\mathbf{y}}(t) & =\sum_{p=1}^{P} \mathbf{a}\left(\theta_{p}\right) s_{p}(t)+\mathbf{z}(t) \\
& =\mathbf{A}(\boldsymbol{\theta}) \mathbf{s}(t)+\mathbf{z}(t)
\end{aligned}
$$

where $\mathbf{A}(\boldsymbol{\theta})=\left[\mathbf{a}\left(\theta_{1}\right) \mathbf{a}\left(\theta_{2}\right) \cdots \mathbf{a}\left(\theta_{N}\right)\right]$ is the array steering matrix and

$$
\begin{aligned}
\hat{\mathbf{y}}(t) & =\left[\begin{array}{llll}
\hat{y}_{1}(t) & \hat{y}_{2}(t) & \cdots & \hat{y}_{N}(t)
\end{array}\right] \\
\mathbf{s}(t) & =\left[\begin{array}{llll}
s_{1}(t) & s_{2}(t) & \cdots & s_{P}(t)
\end{array}\right] \\
\mathbf{z}(t) & =\left[\begin{array}{llll}
z_{1}(t) & z_{2}(t) & \cdots & z_{N}(t)
\end{array}\right]
\end{aligned}
$$

The goal is to estimate the arrival angles $\left\{\theta_{p}\right\}_{p=1}^{P}$ from the observed data. Assuming that the signal sources and noise are uncorrelated and that the noise vector is distributed as $\mathbf{z}(t) \sim \mathcal{N}\left(0, \sigma^{2} \mathbf{I}\right)$, the covariance matrix $\mathbf{R}_{\mathrm{yy}}$ of the array observation can be represented as

$$
\begin{aligned}
\mathbf{R}_{\mathrm{yy}} & =\mathbb{E}\left[\mathbf{y}(t) \mathbf{y}^{H}(t)\right] \\
& =\mathbf{A}(\boldsymbol{\theta}) \mathbf{R}_{\mathrm{ss}} \mathbf{A}(\boldsymbol{\theta})^{H}+\mathbf{R}_{\mathrm{zz}}
\end{aligned}
$$

where $\mathbf{R}_{\mathrm{ss}}=\mathbb{E}\left[\mathbf{s}(t) \mathbf{s}(t)^{H}\right]$ and $\mathbf{R}_{\mathrm{zz}}=\mathbb{E}\left[\mathbf{z}(t) \mathbf{z}(t)^{H}\right]$. The Eigenvalue Decompostion (EVD) of $\mathbf{R}_{\mathrm{yy}}$ can be written as

$$
\mathbf{R}_{\mathrm{yy}}=\sum_{n=1}^{N} \lambda_{n} \mathbf{e}_{n} \mathbf{e}_{n}^{H}
$$

where $\lambda_{n}$ and $\mathbf{e}_{n}$ are the $n$th eigenvalue and the corresponding eigenvector, respectively. In an ideal case, the eigenvalues obey

$$
\lambda_{1} \geq \lambda_{2} \geq \cdots \geq \lambda_{P} \geq \lambda_{P+1}=\lambda_{P+2}=\cdots=\lambda_{N}=\sigma^{2}
$$




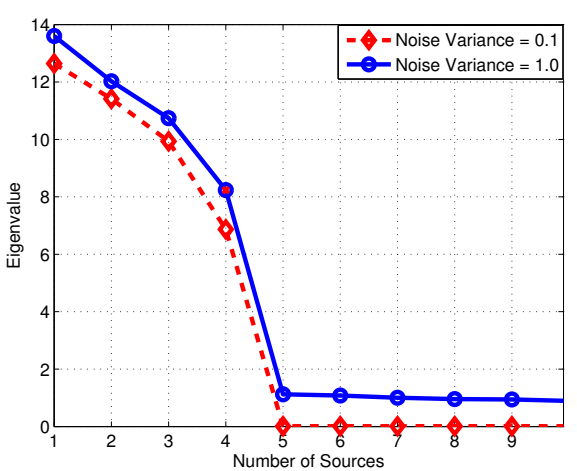

Figure 3.1: The eigenvalues of spatial covariance matrix for a 10element ULA observation data having four sources with DOA = $[20,45,-48,-65]$ and noise variances 0.1 and 1 .

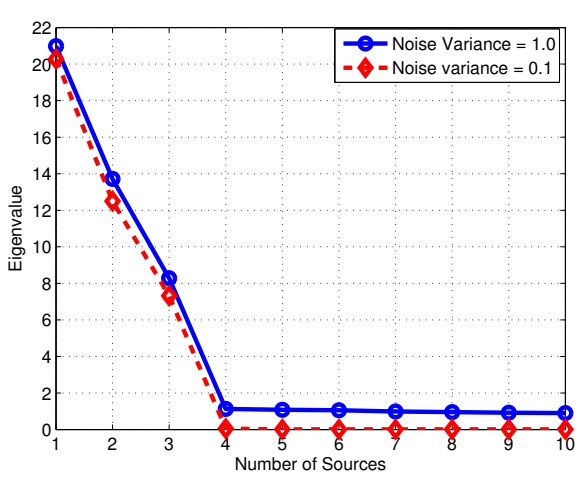

Figure 3.2: The eigenvalues of spatial covariance matrix for a 10element ULA observation data having four sources with DOA = $[20,20.6,-48,-65]$ and noise variances 0.1 and 1 .

However, (3.7) does not always hold in practice. In Figure 3.1 and Figure 3.2, we show the eigenvalues of the data covariance matrix $\mathbf{R}_{\mathrm{yy}}$ for a 10-element ULA measurement. In both cases, there are four sources with different angles of arrival. In Figure 3.1, we observe that the first four eigenvalues are greater than the noise variance and the last six are approximately equal to the noise variance. In Figure 3.2 however, only three eigenvalues are greater than the noise variance. This does not agree with (3.7). An explanation for this is that the two closely spaced sources are seen as one. In general, the EVD of $\mathbf{R}_{\mathrm{yy}}$ can be expressed as

$$
\begin{aligned}
\mathbf{R}_{\mathrm{yy}} & =\left[\begin{array}{ll}
\mathbf{E}_{\mathrm{s}} & \mathbf{E}_{\mathrm{n}}
\end{array}\right]\left[\begin{array}{ll}
\boldsymbol{\Lambda}_{\mathrm{s}} & \\
& \boldsymbol{\Lambda}_{\mathrm{n}}
\end{array}\right]\left[\begin{array}{l}
\mathbf{E}_{\mathrm{s}}^{H} \\
\mathbf{E}_{\mathrm{n}}^{H}
\end{array}\right] \\
& =\mathbf{E}_{\mathrm{s}} \boldsymbol{\Lambda}_{\mathrm{s}} \mathbf{E}_{\mathrm{s}}^{H}+\mathbf{E}_{\mathrm{n}} \boldsymbol{\Lambda}_{\mathrm{n}} \mathbf{E}_{\mathrm{n}}^{H}
\end{aligned}
$$

where $\mathbf{E}_{\mathrm{s}} \in \mathbb{C}^{N \times P}$ and $\Lambda_{\mathrm{s}} \in \mathbb{R}^{P \times P}$ are the signal subspace eigenvectors and the associated eigenvalues, respectively. $\mathbf{E}_{\mathrm{n}} \in \mathbb{C}^{N \times(N-P)}$ and $\Lambda_{\mathrm{n}} \in$ $\mathbb{R}^{(N-P) \times(N-P)}$ corresponds to the noise eigenvectors and eigenvalues. It 
should be noted that the signal and noise subspace eigenvectors are orthogonal to each other, i.e., $\mathbf{e}_{n 1} \mathbf{e}_{n 2}^{H}=0 ; \forall n 1 \in\{1, \ldots, P\}, n 2 \in\{P+1, \ldots, M\}$.

\subsection{Subspace Based Methods}

In this section, we present a review of the subspace based parameter estimation methods.

\subsubsection{MUSIC}

MUSIC [168] exploits the orthogonality of the signal and noise subspace for model parameter estimation. The fundamental idea is that the array steering vector $\mathbf{a}(\theta)$ in the direction of a source is orthogonal to the noise subspace and hence, the noise eigenvectors. This results in peaks in the plot of the MUSIC pseudo-spectrum, defined as [168, 183]

$$
\mathbf{P}_{\mathrm{MUSIC}}=\frac{1}{\mathbf{a}(\boldsymbol{\theta})^{H} \mathbf{E}_{\mathrm{n}} \mathbf{E}_{\mathrm{n}}^{H} \mathbf{a}(\boldsymbol{\theta})}
$$

or

$$
\mathbf{P}_{\text {MUSIC }}=\frac{\mathbf{a}(\boldsymbol{\theta})^{H} \mathbf{a}(\boldsymbol{\theta})}{\mathbf{a}(\boldsymbol{\theta})^{H} \mathbf{E}_{\mathrm{n}} \mathbf{E}_{\mathrm{n}}^{H} \mathbf{a}(\boldsymbol{\theta})}
$$

The parameter estimates are obtained either by manually locating the peaks of (3.9) or using a search algorithm to identify the peaks. A plot of the MUSIC Pseudo-spectrum in (3.9) is shown in Figure 3.3. The data is generated synthetically for a 10-element ULA with four sources having AOAs 20, 45, -40 and $-10 \mathrm{deg}$.

\subsubsection{Root MUSIC}

The performance of the MUSIC algorithm and its suitability for practical applications is limited by a number of factors. The estimation performance is limited by the spacing between values at which (3.9) or (3.10) is computed. Furthermore, a comprehensive search scheme is required to locate the peaks 
and evaluate the parameter estimates. This poses a high computational burden, especially for multidimensional problems which will be introduced in the next chapter.

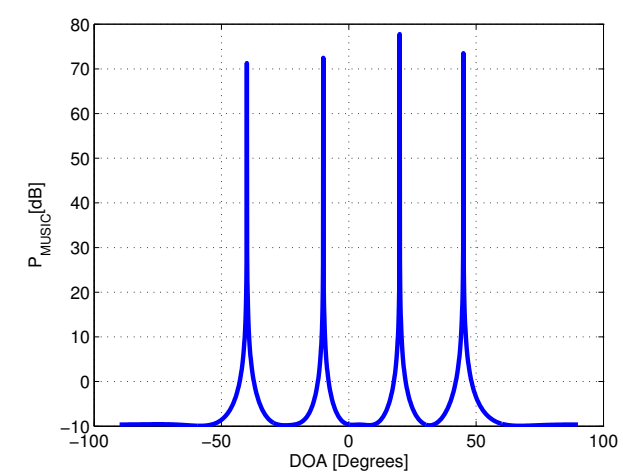

Figure 3.3: MUSIC Pseudospectrum for DOA estimation using a 10element ULA at $\mathrm{SNR}=6 \mathrm{~dB}$. The true values of the DOA are 20, 45, -40 and $-10 \mathrm{deg}$.

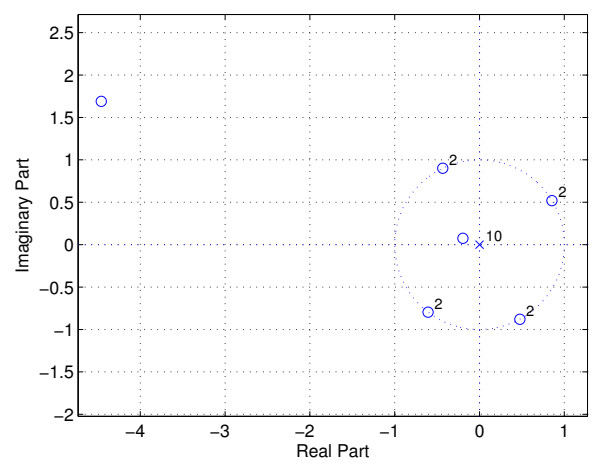

Figure 3.4: The roots of the RootMUSIC function in (3.14) for DOA estimation using a 6-element ULA at $\mathrm{SNR}=6 \mathrm{~dB}$. The true values of the DOA are $20,45,-40$ and $-10 \mathrm{deg}$.

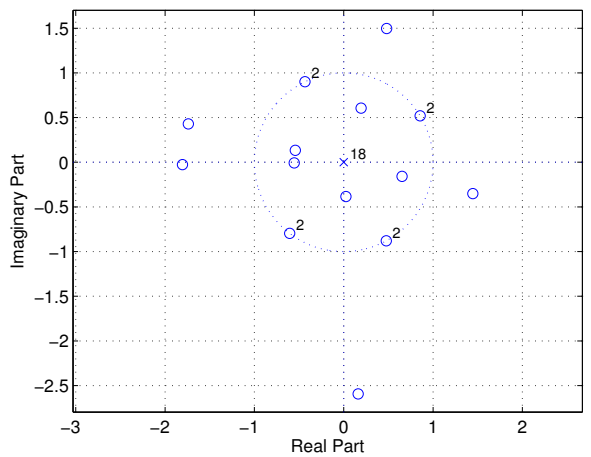

Figure 3.5: The roots of the RootMUSIC function in (3.14) for DOA estimation using a 10-element ULA at $\mathrm{SNR}=6 \mathrm{~dB}$. The true values of the DOA are $20,45,-40$ and $-10 \mathrm{deg}$.

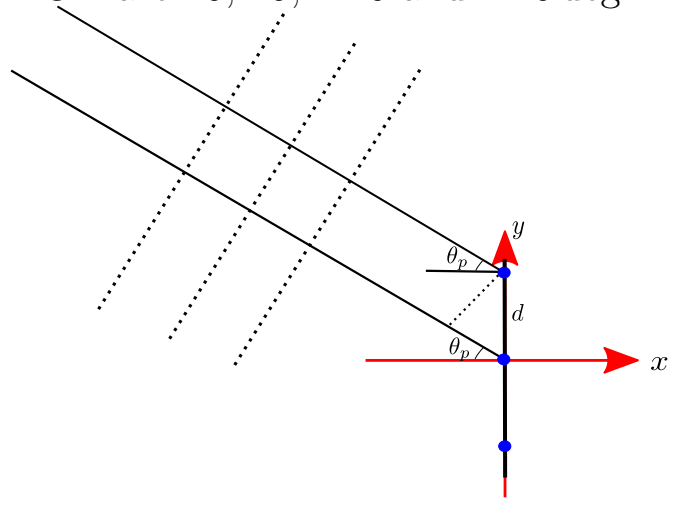

Figure 3.6: Illustration of Signal Measurement using uniform linear array with equal spacing between elements 
To overcome these limitations, Root-MUSIC was proposed as a modified version of MUSIC based on polynomial root finding [34]. Root-MUSIC has been shown to increase the resolution and decrease the computational complexity of MUSIC algorithm. Root-MUSIC is a model based algorithm which directly estimates the parameters using a model of the array steering vector and the received measured data.

Consider an N-element ULA with spacing $d$ (in wavelengths) between antenna elements as shown in Fig. 3.6. Let

$$
z_{p}=\exp \left(j 2 \pi d \sin \theta_{p}\right), \quad p=1,2, \cdots, P
$$

The array steering vector for the $p$ th impinging signal can therefore be expressed as

$$
\mathbf{a}\left(\theta_{p}\right)=\left[1, z_{p}, z_{p}^{2}, \cdots, z_{p}^{(N-1)}\right]
$$

The MUSIC spectrum in (3.9) can be written as

$$
\mathbf{P}_{\text {MUSIC }}^{-1}(\theta)=\mathbf{a}(\theta)^{H} \mathbf{E}_{\mathrm{n}} \mathbf{E}_{\mathrm{n}}^{H} \mathbf{a}(\theta)
$$

Substituting (3.12) into (3.13) and simplifying yields the polynomial

$$
\begin{aligned}
\mathbf{D}(z) & =\mathbf{a}^{T}(1 / z) \mathbf{C a}(z) \\
& =\sum_{n=-(N-1)}^{(N-1)} \mathbf{C}_{n} z^{n}
\end{aligned}
$$

where $\mathbf{C}=\mathbf{E}_{\mathrm{n}} \mathbf{E}_{\mathrm{n}}^{H}$ and $\mathbf{C}_{n}$ denotes the sum of the elements on the $n$th diagonal of $\mathbf{C}$. $\mathbf{D}(z)$ is a polynomial of degree $2(N-1)$ and therefore has $2(N-1)$ zeros, which form conjugate reciprocal pairs with one zero lying within the unit circle and the other outside. Due to measurement noise effects, the location of the roots on the unit circle may be distorted and the parameters are estimated from the $P$ closest roots to the unit circle. Plots of the roots of (3.14) are presented in Figure 3.4 and Figure 3.5 for a 6-element and 10-element ULA observation, respectively. We observe in both cases that four pairs of roots corresponding to the parameter estimates lie very close to the unit circle. The roots can be used in (3.11) to obtain estimates of $\theta_{p}$. 


\subsubsection{ESPRIT}

The ESPRIT algorithm [159] is another subspace method and possibly the most popular in channel prediction studies. Compared to MUSIC, it offers more robustness against array imperfections and since it does not require a search for peaks, it has significantly lower computational complexity and storage requirements. However, it depends on shift invariance structure of the array obtained by creating two overlapping sub-arrays from the original array with a constant translational distance. Consider the ULA in Fig. 3.6. Using (3.12), the Vandermonde structured array steering matrix is obtained as

$$
\mathbf{A}=\left[\begin{array}{cccc}
1 & 1 & \cdots & 1 \\
z_{1} & z_{2} & \cdots & z_{P} \\
\vdots & \vdots & \ddots & \vdots \\
z_{1}^{N-1} & z_{2}^{N-1} & \cdots & z_{P}^{N-1}
\end{array}\right] \in \mathbb{C}^{N \times P}
$$

Consider the steering matrices for two subarrays depicted in Fig. 3.7 with maximum overlap (i.e., translational distance of $d$ )

$$
\mathbf{A}_{1}=\left[\begin{array}{cccc}
1 & 1 & \cdots & 1 \\
z_{1} & z_{2} & \cdots & z_{P} \\
\vdots & \vdots & \ddots & \vdots \\
z_{1}^{N-2} & z_{2}^{N-2} & \cdots & z_{P}^{N-2}
\end{array}\right] \in \mathbb{C}^{(N-1) \times P}
$$

and

$$
\mathbf{A}_{2}=\left[\begin{array}{cccc}
z_{1} & z_{2} & \cdots & z_{P} \\
z_{1}^{2} & z_{2}^{2} & \cdots & z_{P}^{2} \\
\vdots & \vdots & \ddots & \vdots \\
z_{1}^{N-1} & z_{2}^{N-1} & \cdots & z_{P}^{N-1}
\end{array}\right] \in \mathbb{C}^{(N-1) \times P}
$$

Note that $\mathbf{A}_{1}$ and $\mathbf{A}_{2}$, corresponds to $\mathbf{A}$ without the first and the last rows, respectively, and are related by the invariance equation

$$
\mathbf{A}_{1}=\mathbf{A}_{2} \Phi
$$


where $\boldsymbol{\Phi}$ is the diagonal matrix

$$
\boldsymbol{\Phi}=\left[\begin{array}{cccc}
z_{1} & 0 & \cdots & 0 \\
0 & z_{2} & \cdots & 0 \\
\vdots & \vdots & \ddots & z_{P}
\end{array}\right]
$$

The diagonal entries of $\boldsymbol{\Phi}$ corresponds to the phase shift of each of the $\mathrm{P}$ signal sources from one element of the array to its immediate neighbour. As seen from (3.20), once $\boldsymbol{\Phi}$ is known, the signal parameters can be estimated using (3.11). However, matrices $\mathbf{A}_{1}, \mathbf{A}_{2}$ and hence, $\boldsymbol{\Phi}$ are unknown. Since the columns of $\mathbf{A}$ and $\mathbf{E}_{\mathrm{s}}$ both span the signal subspace, they can be related by

$$
\mathbf{E}_{\mathrm{s}}=\mathrm{AT}
$$

where $\mathbf{T} \in \mathbb{C}^{N \times N}$ is an invertible subspace rotation matrix. Let $\mathbf{E}_{\mathrm{s} 1}$ and $\mathbf{E}_{\mathrm{s} 2}$ be submatrices formed from $\mathbf{E}_{\mathrm{s}}$ similar to $\mathbf{A}_{1}$ and $\mathbf{A}_{2}$. The ESPRIT algorithm begins by forming the invariance equation ${ }^{1}$

$$
\mathrm{E}_{\mathrm{s} 1}=\mathbf{E}_{\mathrm{s} 2} \Psi
$$

where $\boldsymbol{\Psi}=\mathbf{T}^{-1} \mathbf{\Phi} \mathbf{T}$. Since the covariance matrix $\mathbf{R}_{\mathrm{yy}}$ and hence, $\mathbf{E}_{\mathrm{s}}$ are typically obtained from noisy measured data, (3.22) is solved using the least square method to obtain

$$
\boldsymbol{\Psi}=\left(\mathbf{E}_{\mathrm{s} 1}^{H} \mathbf{E}_{\mathrm{s} 1}\right)^{-1}\left(\mathbf{E}_{\mathrm{s} 1}^{H} \mathbf{E}_{\mathrm{s} 2}\right)
$$

The parameter estimate for the $p$ th signal source is then evaluated using

$$
\theta_{p}=\sin ^{-1}\left[\frac{\arg \left(\lambda_{p}\right)}{2 \pi d}\right]
$$

where $\arg [\cdot]$ denotes the phase angle of the associated complex number on $[0,2 \pi)$ and $\lambda_{p}$ is the $p$ th eigenvalue of $\boldsymbol{\Psi}$. A plot of the $\lambda_{p} ; p=1, \ldots, 4$ is shown in Fig. 3.8 for the 10-element ULA observation. It shows that similar to the Root-MUSIC, the eigenvalues lie on the unit circle $^{2}$.

\footnotetext{
${ }^{1}$ The invariance equation is obtained by making $\mathbf{A}$ the subject in (3.21) and substituting the result into (3.19).

${ }^{2}$ This observation could be used in detecting and eliminating erroneous estimates in cases where the number of sources is not exactly know apriori.
} 


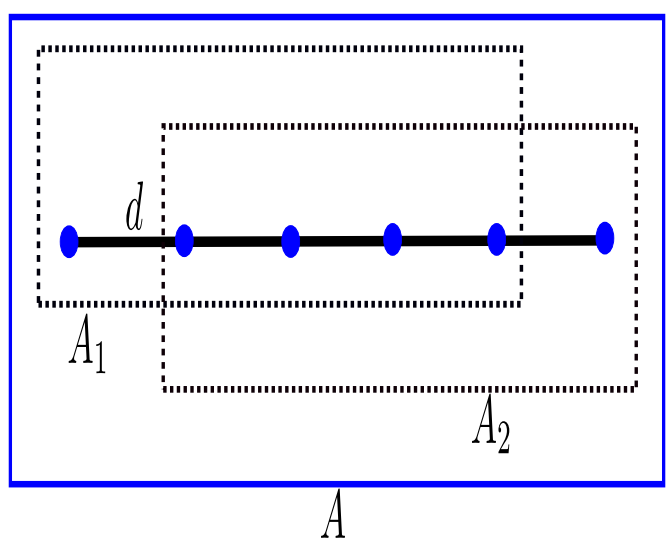

Figure 3.7: Illustration of subarray selection.

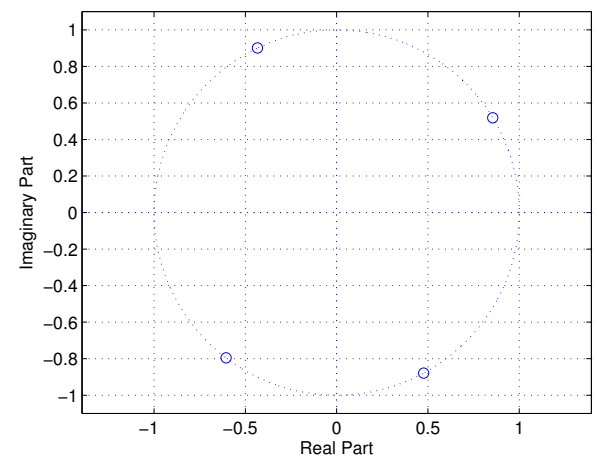

Figure 3.8: The eigenvalues of the solution to the invariance equation in (3.23) for DOA estimation using a 10element ULA at $\mathrm{SNR}=6 \mathrm{~dB}$.

\subsubsection{Unitary ESPRIT}

The Unitary ESPRIT is a simpler and more efficient implementation of the ESPRIT algorithm. The uniqueness of the algorithm is that it is formulated in terms of real-valued computations [73]. However, in addition to the invariance structure requirement in ESPRIT, it requires centro-symmetric antenna arrays. In order to apply Unitary ESPRIT, the covariance matrix is estimated using forward-backward averaging as

$$
\begin{aligned}
\mathbf{R}_{\mathrm{fb}} & =\frac{1}{2} \mathbb{E}\left[\mathbf{y}(t) \mathbf{y}(t)^{H}+\boldsymbol{\Pi}_{N} \mathbf{y}(t)^{*} \mathbf{y}(t)^{T} \boldsymbol{\Pi}_{N}\right] \\
& =\frac{1}{2}\left(\mathbf{R}_{\mathrm{yy}}+\boldsymbol{\Pi}_{N} \mathbf{R}_{\mathrm{yy}}^{*} \boldsymbol{\Pi}_{N}\right)
\end{aligned}
$$


where $[\cdot]^{*}$ denotes matrix conjugate and $\boldsymbol{\Pi}_{N}$ is the exchange matrix, defined as

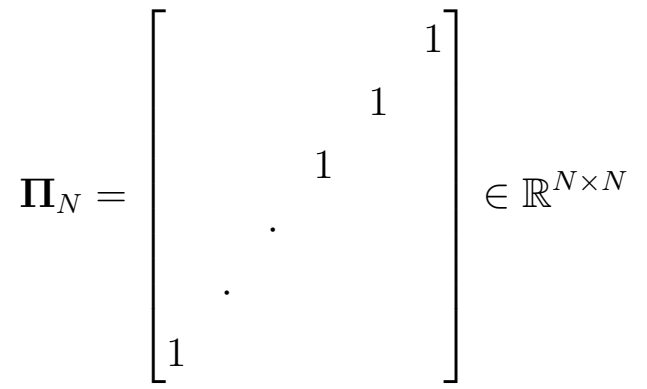

Since the forward-backward averaged covariance matrix in (3.25) is centrosymmetric ${ }^{3}$, it can be transformed to a real valued matrix as [73]

$$
\mathbf{C}=\mathbf{Q}_{N}^{H} \mathbf{R}_{\mathrm{fb}} \mathbf{Q}_{N}
$$

where $\mathbf{Q}_{N}$ is a sparse unitary (or left-real ${ }^{4}$.) matrix defined as

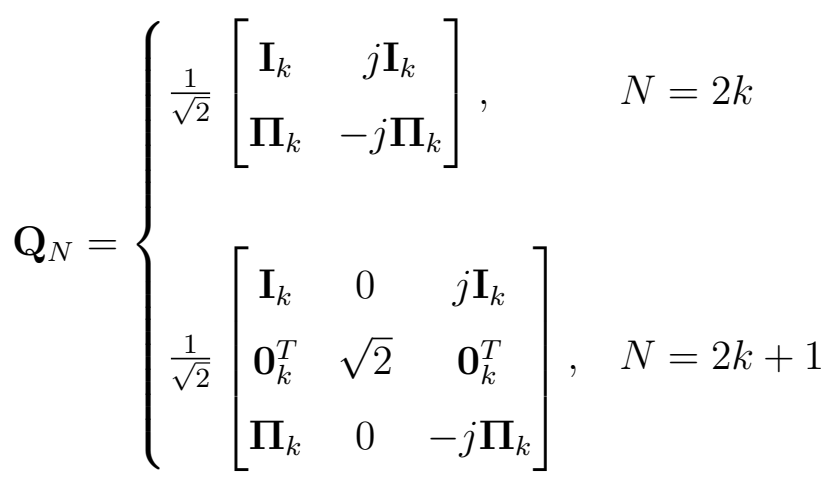

Using the transformation in (3.27), the transformed steering matrix can be shown to be

$$
\mathbf{D}=\mathbf{Q}_{N} \mathbf{A}
$$

and $\mathbf{D}$ satisfies the following invariance equation

$$
\mathbf{K}_{1} \mathbf{D} \Phi=\mathbf{K}_{2} \mathbf{D}
$$

\footnotetext{
${ }^{3} \mathrm{~A} N \times N$ matrix, $\mathbf{A}$, is centrosymmetric if it is symmetric about its center, i.e., the entries of $\mathbf{A}$ satisfy the relation, $\mathbf{A}_{j, k}=\mathbf{A}_{N-j+1, N-k+1}$ for $1 \leq j, k \leq N$. It therefore means that $A$ is centro-symmetric if and only if $\mathbf{A} \Pi_{N}=\Pi_{N} \mathbf{A}$.

${ }^{4} \mathrm{~A}$ complex matrix $\mathbf{Q}$ is left real if $\boldsymbol{\Pi}_{N} \mathbf{Q}^{H} \boldsymbol{\Pi}_{N}=\mathbf{Q}$ [111]
} 
where $\boldsymbol{\Phi}=\operatorname{diag}\left\{\tan \left(\pi d \sin \theta_{p}\right)\right\}_{p=1}^{P} . \quad \mathbf{K}_{1}$ and $\mathbf{K}_{2}$ are transformed selection matrices defined as

$$
\mathbf{K}_{1}=2 \mathfrak{R}\left\{\mathbf{Q}_{P} \mathbf{J}_{2} \mathbf{Q}_{N}\right\}
$$

and

$$
\mathbf{K}_{2}=2 \mathfrak{I}\left\{\mathbf{Q}_{P} \mathbf{J}_{2} \mathbf{Q}_{N}\right\}
$$

where $\mathfrak{R}[\cdot]$ and $\mathfrak{I}[\cdot]$ denote the real part and imaginary part of the associated matrix, respectively and $\mathbf{J}_{2}$ is the selection matrix that selects $\mathbf{A}_{2}$ from $\mathbf{A}$ defined as

$$
\mathbf{J}_{2}=\left[\begin{array}{ll}
\mathbf{0}_{(N-1) \times 1} & \mathbf{I}_{(N-1)}
\end{array}\right]
$$

As before, since $\mathbf{D}$ and $\mathbf{E}_{s}$ span the same signal subspace, a real valued invariance equation can be formed as

$$
\mathbf{K}_{1} \mathbf{E}_{s} \boldsymbol{\Psi}=\mathbf{K}_{2} \mathbf{E}_{s}
$$

where $\boldsymbol{\Psi}=\mathbf{T} \boldsymbol{\Phi} \mathbf{T}^{-1}$. After solving (3.34) for $\boldsymbol{\Psi}$ using the least square approach, the parameter for the $p$ th signal source is computed using

$$
\theta_{p}=\sin ^{-1}\left\{\frac{\tan ^{-1}\left[\lambda_{p}\right]}{\pi d}\right\}
$$

\subsubsection{Matrix Pencil}

The subspace methods described in Sections 3.2.1-3.2.4 depend on the EVD of the covariance matrix. The computation requirement for estimating the covariance matrix can be eliminated by operating directly on the data $\mathbf{y}(t)$. A Matrix Pencil (MP) method similar to the ESPRIT algorithm but without covariance matrix estimation is proposed in [83]. Expressing the data from the $n$th element of the ULA as

$$
y_{n}(t)=\sum_{p=1}^{P} s_{p}(t) z_{p}^{n-1}
$$


where $z_{p}$ is defined in (3.11), the data from the $N$ antenna elements can be arranged in a $\left(N-M_{\mathrm{f}}\right) \times\left(M_{\mathrm{f}}+1\right)$ matrix

$$
\mathbf{Y}=\left[\begin{array}{cccc}
y_{1} & y_{2} & \cdots & y_{M_{\mathrm{f}}+1} \\
y_{2} & y_{3} & \cdots & y_{M_{\mathrm{f}}+2} \\
\vdots & \vdots & \ddots & \vdots \\
y_{N-M_{\mathrm{f}}} & y_{N-M_{\mathrm{f}}+1} & \cdots & y_{N}
\end{array}\right]
$$

where $M_{\mathrm{f}}$ is the pencil (free) parameter which is chosen such that ${ }^{5}$

$$
\begin{array}{r}
P \leq M_{\mathrm{f}} \leq N-M_{\mathrm{f}} \quad N \text { even } \\
P \leq M_{\mathrm{f}} \leq N-M_{\mathrm{f}}+1 \quad N \text { odd }
\end{array}
$$

Using (3.36), Y can be written as

$$
\mathbf{Y}=\left[\begin{array}{cccc}
\sum_{p=1}^{P} s_{p} & \sum_{p=1}^{P} s_{p} z_{p} & \cdots & \sum_{p=1}^{P} s_{p} z_{p}^{M_{\mathrm{f}}} \\
\sum_{p=1}^{P} s_{p} z_{p} & \sum_{p=1}^{P} s_{p} z_{p}^{2} & \cdots & \sum_{p=1}^{P} s_{p} z_{p}^{M_{\mathrm{f}}+1} \\
\vdots & \vdots & \ddots & \vdots \\
\sum_{p=1}^{P} s_{p} z_{p}^{N-M_{\mathrm{f}}-1} & \sum_{p=1}^{P} s_{p} z_{p}^{N-M_{\mathrm{f}}} & \cdots & \sum_{p=1}^{P} s_{p} z_{p}^{N-1}
\end{array}\right]
$$

Two $\left(N-M_{\mathrm{f}}\right) \times M_{\mathrm{f}}$ matrices $\mathbf{Y}_{0}$ and $\mathbf{Y}_{1}$ are then formed from $\mathbf{Y}$,

$$
\mathbf{Y}_{0}=\left[\begin{array}{cccc}
y_{1} & y_{2} & \cdots & y_{M_{\mathrm{f}}} \\
y_{2} & y_{3} & \cdots & y_{M_{\mathrm{f}}+1} \\
\vdots & \vdots & \ddots & \vdots \\
y_{N-M_{\mathrm{f}}} & y_{N-M_{\mathrm{f}}+1} & \cdots & y_{N-1}
\end{array}\right]
$$

and

$$
\mathbf{Y}_{1}=\left[\begin{array}{cccc}
y_{2} & y_{3} & \cdots & y_{M_{\mathrm{f}}+1} \\
y_{3} & y_{4} & \cdots & y_{M_{\mathrm{f}}+2} \\
\vdots & \vdots & \ddots & \vdots \\
y_{N-M_{\mathrm{f}}+1} & y_{N-M_{\mathrm{f}}+2} & \cdots & y_{N}
\end{array}\right]
$$

\footnotetext{
${ }^{5} M_{\mathrm{f}}$ is commonly chosen between $N / 2$ and $N / 3$ for efficient noise filtering [164].
} 
Similar to the ESPRIT invariance equation (3.19), the MP approach is based on forming two equations from (3.40) and (3.41), thus

$$
\begin{aligned}
& \mathbf{Y}_{0}=\mathbf{Z}_{1} \mathbf{D}(\mathbf{s}) \mathbf{Z}_{2} \\
& \mathbf{Y}_{1}=\mathbf{Z}_{1} \mathbf{D}(\mathbf{s}) \boldsymbol{\Phi} \mathbf{Z}_{2}
\end{aligned}
$$

where

$$
\begin{gathered}
\mathbf{Z}_{1}=\left[\begin{array}{ccccc}
1 & & 1 & \cdots & 1 \\
z_{1} & & z_{2} & \cdots & z_{P} \\
\vdots & \vdots & \ddots & \vdots \\
z_{1}^{N-M_{\mathrm{f}}-1} & z_{2}^{N-M_{\mathrm{f}}-1} & \cdots & z_{P}^{N-M_{\mathrm{f}}-1}
\end{array}\right] \\
\mathbf{Z}_{2}=\left[\begin{array}{cccc}
1 & z_{1} & \cdots & z_{1}^{M_{\mathrm{f}}-1} \\
1 & z_{2} & \cdots & z_{2}^{M_{\mathrm{f}}-1} \\
\vdots & \vdots & \ddots & \vdots \\
1 & z_{P} & \cdots & z_{P}^{M_{\mathrm{f}}-1}
\end{array}\right] \\
\mathbf{D}(\mathbf{s})=\left[\begin{array}{cccc}
s_{1} & 0 & \cdots & 0 \\
0 & s_{2} & \cdots & 0 \\
\vdots & \vdots & \ddots & \vdots \\
0 & 0 & \cdots & s_{P}
\end{array}\right]
\end{gathered}
$$

and $\boldsymbol{\Phi}$ is given in (3.20). In the absence of noise, it can be shown that the submatrices $\mathbf{Y}_{0}$ and $\mathbf{Y}_{1}$ have rank $P$, provided that $M_{\mathrm{f}}$ satisfies the selection criteria in (3.38) [164]. Now consider the Matrix Pencil

$$
\mathbf{Y}_{1}-\lambda \mathbf{Y}_{0}=\mathbf{Z}_{1} \mathbf{D}(\boldsymbol{\alpha})\left[\Phi-\lambda \mathbf{I}_{P}\right] \mathbf{Z}_{2}
$$

where $\mathbf{I}_{p}$ is a $P \times P$ identity matrix. For arbitrary values of $\lambda,(3.47)$ has rank $P$. If $\lambda=z_{p}, p=1, \cdots, P$, the $p$ th row of $\boldsymbol{\Phi}-\lambda \mathbf{I}_{P}$ is zero and the rank reduces to $P-1$. This implies that the parameter set $\left\{z_{p}\right\}_{p=1}^{P}$ are the generalized eigenvalues of the matrix pair $\left[\mathbf{Y}_{0}, \mathbf{Y}_{1}\right]$ which can be obtained as the eigenvalues of

$$
\left(\mathbf{Y}_{0}^{H} \mathbf{Y}_{0}\right)^{-1} \mathbf{Y}_{0}^{H} \mathbf{Y}_{1}
$$

The parameter estimates are then obtained using (3.24). 


\subsection{Adaptive Subspace Methods}

In practice, the data covariance matrix in (3.8) is estimated from the available noisy samples as

$$
\hat{\mathbf{R}}_{\mathrm{yy}}=\frac{1}{K} \sum_{k=1}^{K} \hat{\mathbf{y}}(k) \hat{\mathbf{y}}(k)^{H}
$$

where we have have replaced the continuous time variable $t$ in (3.1) with the discrete index $k$. $\hat{\mathbf{R}}$ is an asymptotic unbiased estimate of $\mathbf{R}$ (i.e., it approaches the actual value as $K$ increases). The computation in (3.49) has complexity of order $\mathcal{O}\left(N^{3}\right)$ making repeated computation unattractive for practical applications. The computational load may be reduced by updating the covariance matrix recursively as new measurements are taken

$$
\hat{\mathbf{R}}_{\mathrm{yy}}(k+1)=\frac{1}{k+1}\left(k \hat{\mathbf{R}}_{\mathrm{yy}}(k)+\hat{\mathbf{y}}(k+1) \hat{\mathbf{y}}(k+1)^{H}\right)
$$

Since multipath parameters are slowly time-varying in practice, it is often necessary to periodically update the covariance matrix such that older observations are de-weighted. This can be achieved by formulating (3.50) as a first order moving average filter. The updated matrix at the $(k+1)$ th instant is thus

$$
\hat{\mathbf{R}}_{\mathrm{yy}}(k+1)=\alpha \hat{\mathbf{R}}_{\mathrm{yy}}(k)+(1-\alpha) \hat{\mathbf{y}}(k+1) \hat{\mathbf{y}}(k+1)^{H}
$$

where $\alpha$ denotes the forgetting factor, $0<\alpha<1$. With a proper choice of $\alpha$, subspace methods based on the EVD of (3.51) can therefore track the slow variation of the parameters over time. The computational efficiency and accuracy of the estimation can be improved by using iterative subspace tracking algorithms such as Bi-iteration Singular Value Decomposition (SVD) $[155,185,186]$ and Projection Approximation Subspace Tracking (PAST) [210, 212]. A brief overview of PAST [212] and its extension - PAST with deflation (PASTD) [211] is presented in the following section. 


\subsubsection{Projection Approximation Subspace Tracking (PAST)}

The PAST algorithm for principal subspace tracking was first proposed by Yang [212]. The basic idea is the interpretation of the signal subspace as the solution of an unconstrained minimization problem. It involves minimising an exponentially weighted cost function defined as [212]

$$
\begin{aligned}
J(\mathbf{W}(k))= & \sum_{i=1}^{k} \alpha^{k-1}\left\|\mathbf{h}(i)-\mathbf{W}(k) \mathbf{W}^{H}(k) \mathbf{h}(i)\right\|^{2} \\
= & \operatorname{tr}[\mathbf{C}(k)]-2 \operatorname{tr}\left[\mathbf{W}^{H}(k) \mathbf{C}(k) \mathbf{W}(k)\right] \\
& \quad+\operatorname{tr}\left[\mathbf{W}^{H}(k) \mathbf{C}(k) \mathbf{W}(k) \mathbf{W}^{H}(k) \mathbf{W}(k)\right]
\end{aligned}
$$

where $\mathbf{W}(k)$ is a weight matrix and $\mathbf{C}(k)$ is an exponentially weighted correlation matrix given by

$$
\mathbf{C}(k)=\sum_{i=1}^{k} \alpha^{k-1} \mathbf{h}(i) \mathbf{h}^{H}(i)=\alpha \mathbf{C}(k-1)+\mathbf{h}(k) \mathbf{h}^{H}(k)
$$

By using the approximation $\mathbf{W}^{H}(k) \mathbf{h}(i) \approx \mathbf{W}^{H}(k-1) \mathbf{h}(i)$, the fourth order cost function in (3.52) reduces to a quadratic cost function defined as

$$
J^{\prime}(\mathbf{W}(k))=\sum_{i=1}^{k} \alpha^{k-1}\left\|\mathbf{h}(i)-\mathbf{W}(k) \mathbf{h}^{\prime}(i)\right\|^{2}
$$

where $\mathbf{h}^{\prime}(i)=\mathbf{W}^{H}(k-1) \mathbf{h}(i)$. The minimization of (3.54) can be achieved using the RLS algorithms [213]. The iterative procedure constituting the PAST subspace tracker is summarized in Algorithm 1. 


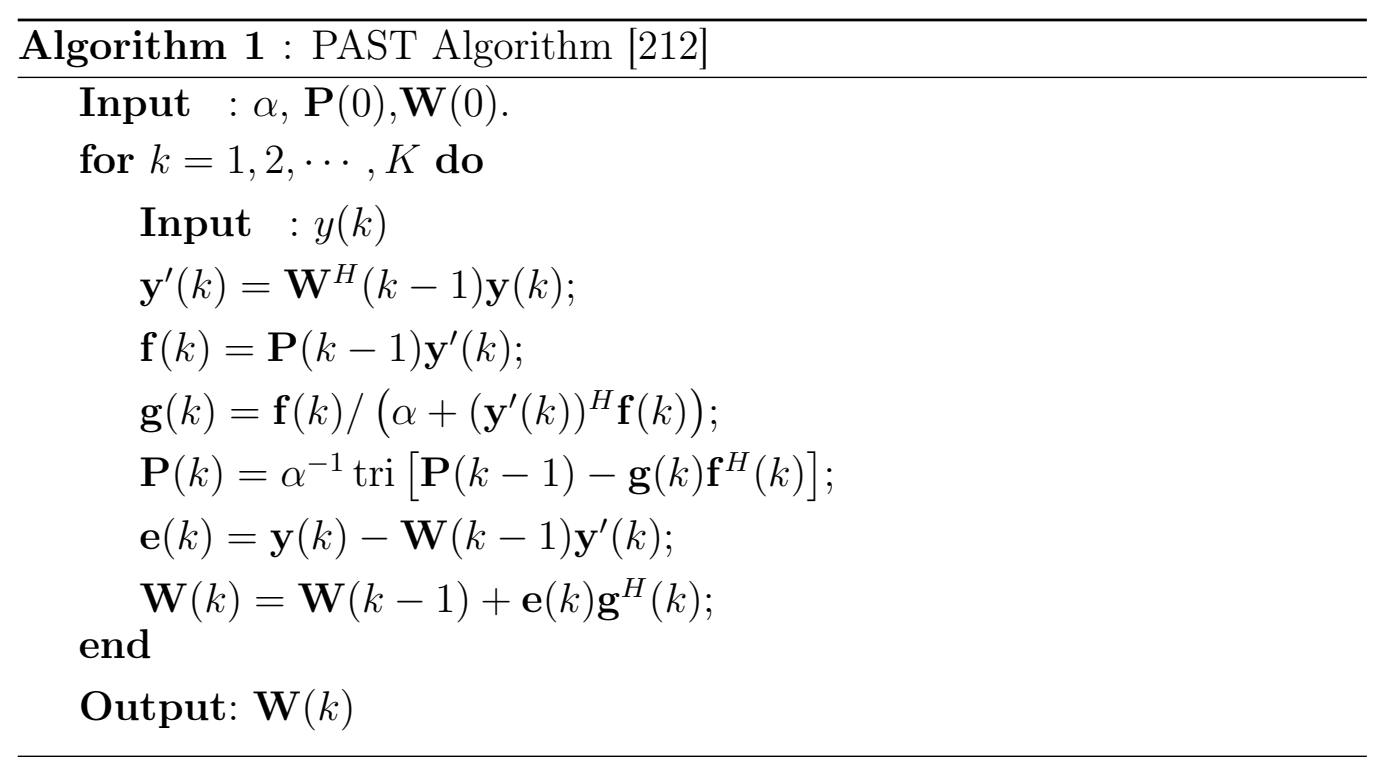

Here, tri[.] means that only the upper (or lower) triangular part of the associated matrix is computed and its Hermitian transpose is copied to the lower (or upper) triangular part. Detailed discussion and proof of the PAST algorithm can be found in [212].

\subsubsection{Projection Approximation Subspace Tracking with Deflation (PASTD)}

PASTD is an extension of the PAST subspace tracker in which the concept of deflation [162] is used to achieve iterative estimation of the signal eigenvectors and eigenvalues [211]. A summary of the PASTD algorithm is given in Algorithm 2. 


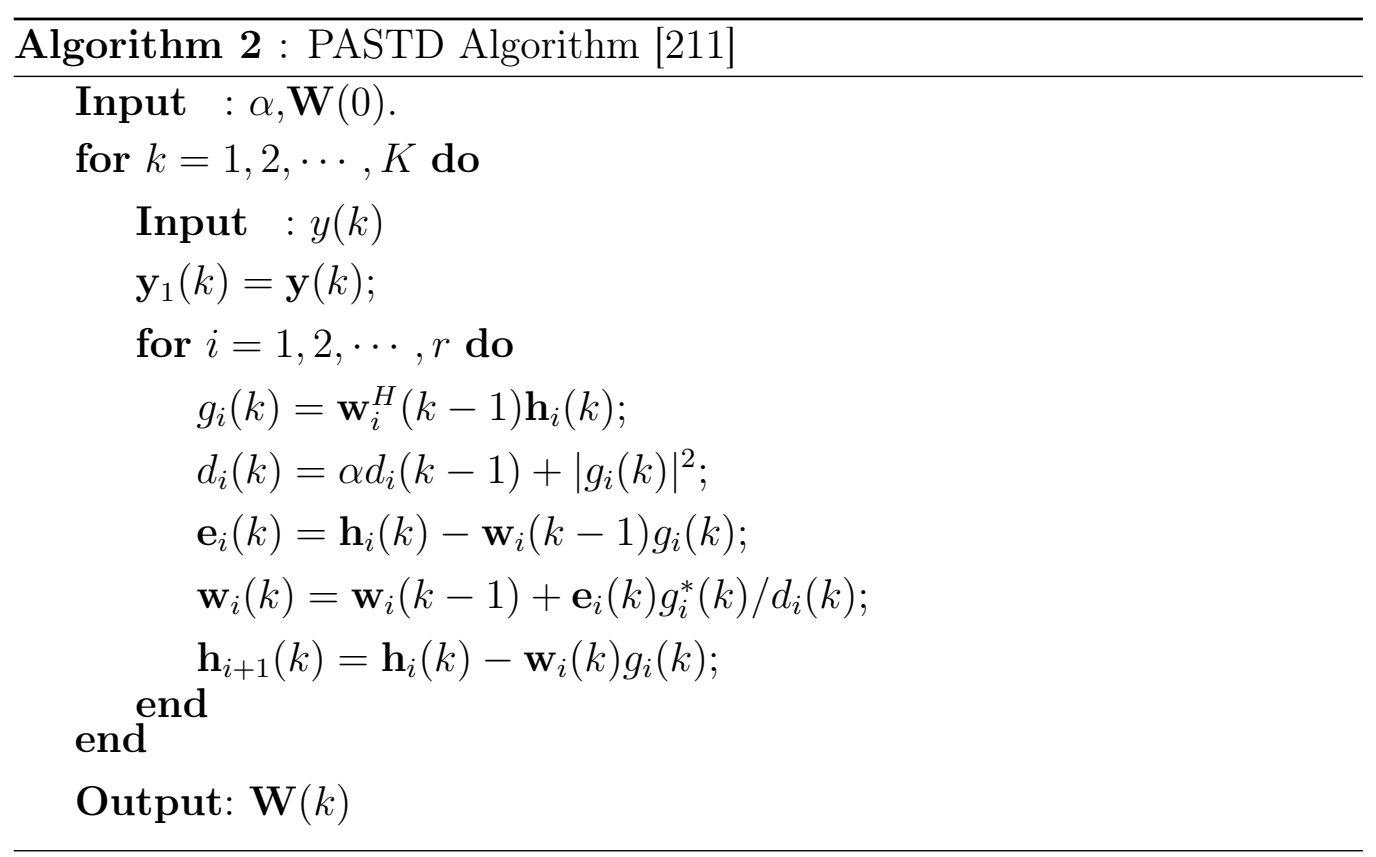

The interested reader may refer to $[211,212]$ for detailed derivation of the PASTD algorithm.

\subsection{Subspace Dimension Estimation}

Estimating the number of dominant scattering sources $P$ is a central problem in parameter estimation and prediction of multipath radio channels. The accuracy of parameter estimation algorithms such as MUSIC and ESPRIT is greatly affected by the number of sources used in for the estimation. In ideal scenarios, noise eigenvalues are equal and can be used for the estimation of the number of signal sources. However, due to noise and error in the estimation of the covariance matrix, the noise eigenvalues, while of similar magnitudes, are no longer equal. Two information theoretic criteria based techniques that are commonly used are the Minimum Description Length (MDL) [199] and Akaike Information Criterion (AIC) [20]. In these techniques $P$ is chosen to minimize some functions which are defined in terms of the ratio of the 
geometric and arithmetic mean of the eigenvalues. These techniques and their variants will be discussed in the following section.

\subsubsection{Minimum Description Length (MDL)}

In the MDL based approach [199], the number of signal sources $P$ is estimated as the value of $p \in\{0,1, \cdots, N-1\}$ that minimizes the MDL criterion

$$
\operatorname{MDL}(p)=-K(N-p) \log \left\{\frac{\left[\prod_{n=p+1}^{N} \lambda_{n}\right]^{\frac{1}{(N-p)}}}{\frac{1}{(N-p)} \sum_{n=p+1}^{N} \lambda_{n}}\right\}+P_{\mathrm{MDL}}(p)
$$

where $\lambda_{n}$ are the eigenvalues of the data covariance matrix $\mathbf{R}_{\mathrm{yy}}$ and $P_{\mathrm{MDL}}(p)=$ $\frac{p}{2}(2 N-p) \log K$ is the MDL penalty factor. Other modified versions of the penalty factor [208] are shown in Table 3.1.

\subsubsection{Akaike Information Criterion (AIC)}

Similar to the MDL based approach, the AIC determines the number of sources by minimizing the AIC criterion

$$
\operatorname{AIC}(p)=-K(N-p) \log \left\{\frac{\left[\prod_{n=p+1}^{N} \lambda_{n}\right]^{\frac{1}{(N-p)}}}{\frac{1}{(N-p)} \sum_{n=p+1}^{N} \lambda_{n}}\right\}+P_{\mathrm{AIC}}(p)
$$

where $P_{\text {AIC }}(p)=p(2 N-p)$ is the AIC penalty factor, modified versions of which are also presented in Table 3.1. Note that although the two methods look very similar, they may produce different estimates. The MDL approach has been shown to provide more accurate estimates than the AIC [201].

\subsubsection{Minimum Mean Square Error - MDL}

The minimum mean square error (MMSE) based MDL [85] improves the performance of the classical MDL based source number estimation method by using mutual information to combine the probability distribution function (PDF) and MMSE between the transmitted and measured signal. The 
Table 3.1: Modified Versions of the AIC and MDL Penalty Functions

\begin{tabular}{lll}
\hline Covariance Estimation Method & $P_{\mathrm{MDL}}(p)$ & $P_{\mathrm{AIC}}(p)$ \\
\hline Real Forward only & $\frac{p}{2}(2 N-2 p+1) \log K$ & $p(2 N-2 p+1)$ \\
Real Forward-Backward & $\frac{p}{2}(N+p+1) \log K$ & $p(N+p+1)$ \\
Complex Forward only & $\frac{p}{2}(2 N-p) \log K$ & $p(2 N-p)$ \\
Complex Forward-Backward & $\frac{p}{4}(2 N-p+1) \log K$ & $\frac{p}{2}(2 N-2 p+1)$ \\
\hline
\end{tabular}

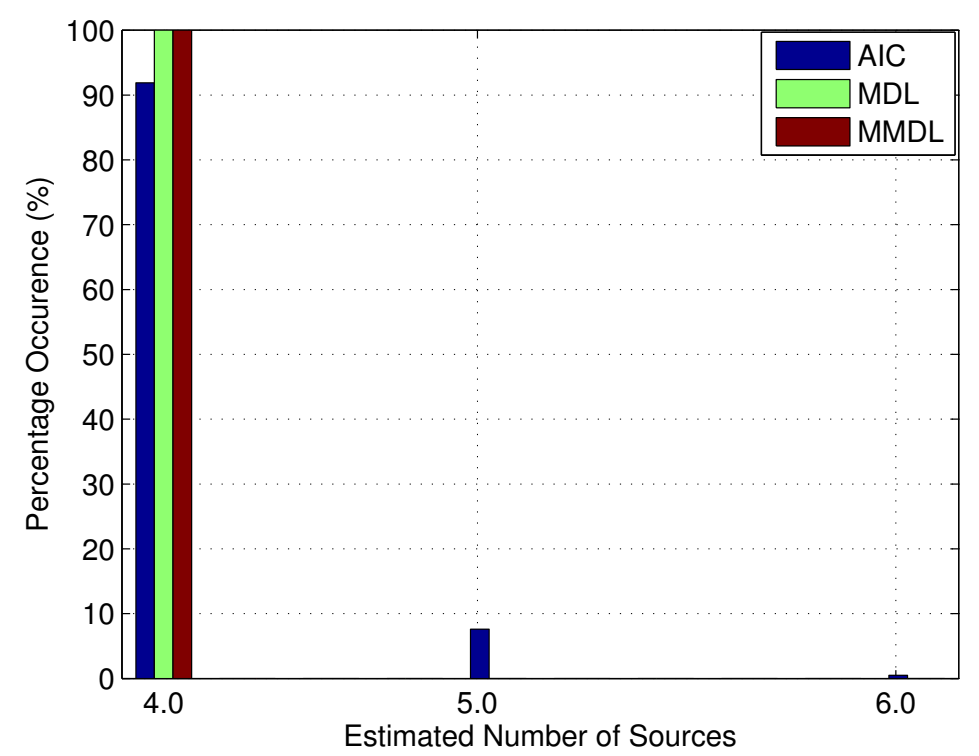

Figure 3.9: Histogram of AIC and MDL estimates of number of sources for a 4 path signal received at a 10 -elements uniform linear array. $\mathrm{SNR}=-10 \mathrm{~dB}$.

estimate of the number of signal sources is given by

$$
\hat{P}=\arg \min _{p=1, \ldots, N-1} \operatorname{MMDL}(p)
$$

where the MMSE-MDL criterion $\operatorname{MMDL}(p)$ is defined as [85]

$$
\operatorname{MMDL}(p)=K \log \left(\lambda_{p}\right)+\frac{1}{2}\left(p^{2}+p\right) \log K
$$


A more robust version of the MMSE-MDL without eigendecomposition was proposed in [84]. The histogram of estimated number of signal using the MDL, AIC and MMDL for a ULA observation data with four sources generated using (3.1) is shown in Figure 3.9. We observe that the MDL and MMDL yield more accurate and consistent estimates compared to the AIC, where the overestimation is approximately $8 \%$. We will use the MDL and MMDL for our studies in later chapters of this thesis.

\subsection{Parameter Estimation for Linear Models}

Consider the problem of estimating the parameter set $\mathbf{s}=\left[\begin{array}{lll}s_{1} & \cdots & s_{P}\end{array}\right]^{T}$ from a linear observation model defined as

$$
\mathbf{y}=\mathbf{A s}+\mathbf{z}
$$

where $\mathbf{A} \in \mathbb{C}^{K \times P}$ is the observation matrix. The goal is to find an estimate $\hat{\mathbf{s}}$ such that $\mathbf{A} \hat{\mathbf{s}}$ is the best approximation of $\mathbf{y}$. A summary of the Least Square (LS) and Minimum Mean Square Error (MMSE) methods for finding the solution of (3.59) is presented next.

\subsubsection{LS Estimation}

Assuming that $\mathbf{A}$ is known and deterministic, the LS solution of (3.59) is defined as

$$
\hat{\mathbf{s}}_{\mathrm{LS}}=\arg \min _{\mathbf{s}}\|\mathbf{y}-\mathbf{A} \mathbf{s}\|^{2}
$$

Differentiating the cost function of (3.60) gives

$$
\frac{\partial\left(\|\mathbf{y}-\mathbf{A} \mathbf{s}\|^{2}\right)}{\partial \mathbf{s}}=-2 \mathbf{A}^{H} \mathbf{y}+2 \mathbf{A}^{H} \mathbf{A} \mathbf{s}
$$

By setting (3.61) equal to zero and solving for $\mathbf{s}$, the LS estimate is obtained as

$$
\begin{aligned}
\hat{\mathbf{s}}_{\mathrm{LS}} & =\left(\mathbf{A}^{H} \mathbf{A}\right)^{-1} \mathbf{A}^{H} \mathbf{y} \\
& =\mathbf{A}^{\dagger} \mathbf{y}
\end{aligned}
$$


where $[\cdot]^{\dagger}$ denotes the Pseudoinverse of the associated matrix.

\subsubsection{MMSE Estimation}

Supposing $\mathbf{A}$ and $\mathbf{s}$ are random, the MMSE estimate of $\mathbf{s}$ is obtained by minimizing the MSE as

$$
\hat{\mathbf{s}}_{\mathrm{MMSE}}=\arg \min _{\mathbf{s}} \mathbb{E}\left[(\mathbf{y}-\mathbf{A} \mathbf{s})^{H}(\mathbf{y}-\mathbf{A} \mathbf{s})\right]
$$

Differentiating the cost function of (3.63) with respect to s yields

$$
\begin{aligned}
\frac{\partial}{\partial \mathbf{s}}\left\{\mathbb{E}\left[(\mathbf{y}-\mathbf{A} \mathbf{s})^{H}(\mathbf{y}-\mathbf{A} \mathbf{s})\right]\right\} & =\mathbb{E}\left[\frac{\partial}{\partial \mathbf{s}}\left\{\mathbf{y}^{H} \mathbf{y}-2 \mathbf{y}^{H} \mathbf{A} \mathbf{s}+\mathbf{s}^{H} \mathbf{A}^{H} \mathbf{A} \mathbf{s}\right\}\right] \\
& =-2 \mathbf{R}_{\mathrm{Ay}}+2 \mathbf{R}_{\mathrm{AA}} \mathbf{s}
\end{aligned}
$$

where $\mathbf{R}_{\mathrm{Ay}}=\mathbb{E}\left\{\mathbf{y}^{H} \mathbf{A}\right\}$ and $\mathbf{R}_{\mathrm{AA}}=\mathbb{E}\left\{\mathbf{A}^{H} \mathbf{A}\right\}$. Equating (3.64) to zero and solving yields

$$
\mathbf{s}_{\mathrm{MMSE}}=\mathbf{R}_{\mathrm{Ay}} \mathbf{R}_{\mathrm{AA}}^{-1}
$$

\subsection{Cramer Rao Bound}

Named after its first authors; H. Cramer and C. R. Rao, the CRB [51, 153] gives a lower bound on the variance of a deterministic parameter estimator [97]. It is generally used in literature as a measure of the best performance that any estimator can achieve and an estimator which attains the CRB is said to be efficient. Note that the formulation of the CRB depends on whether the estimator is biased or unbiased. We will focus on the unbiased $\mathrm{CRB}$ in this section. Consider the parameter estimation problem in (3.1) and let the parameters be denoted by the $P \times 1$ vector

$$
\boldsymbol{\theta}=\left[\begin{array}{llll}
\theta_{1} & \theta_{2} & \cdots & \theta_{P}
\end{array}\right]^{T}
$$


where $[\cdot]^{T}$ denotes the transpose operation. The problem can now be reformulated as

$$
\begin{aligned}
\hat{\mathbf{y}} & =\mathbf{A}(\boldsymbol{\theta}) \mathbf{s}+\mathbf{z} \\
& =\mathbf{y}+\mathbf{z}
\end{aligned}
$$

where $\mathbf{y}$ denotes the noiseless observation. The variance of an unbiased estimate of $\theta_{p}$ is therefore, bounded as

$$
\operatorname{var}\left(\hat{\theta}_{p}\right) \geq \mathfrak{I}_{p p}^{-1}(\boldsymbol{\theta})
$$

where $\mathfrak{J}(\boldsymbol{\theta})$ is the Fisher information matrix (FIM) of $\boldsymbol{\theta}$, and $\mathfrak{J}_{p p}^{-1}(\boldsymbol{\theta})$ denotes the $p$ th diagonal element of the inverse FIM $\mathfrak{J}^{-1}(\boldsymbol{\theta})$. Let the probability distribution function (pdf) of $\mathbf{y}$ be $p(\mathbf{y}: \boldsymbol{\theta})$. The FIM is expressed elementwise as

$$
\mathfrak{J}_{p q}(\boldsymbol{\theta})=-\mathbb{E}\left[\frac{\partial \log p(\mathbf{y}: \boldsymbol{\theta})}{\partial \theta_{p}} \frac{\partial \log p(\mathbf{y}: \boldsymbol{\theta})^{H}}{\partial \theta_{q}}\right]
$$

Assuming that $\mathbf{z} \sim \mathcal{N}(0, \mathbf{C}(\boldsymbol{\theta}))$, such that $\mathbf{y}(\boldsymbol{\theta}) \sim \mathcal{N}(\mathbf{x}(\boldsymbol{\theta}), \mathbf{C}(\boldsymbol{\theta}))$, the FIM is given by the Slepian-Bang's formula [97]

$$
\mathfrak{J}(\boldsymbol{\theta})=\frac{1}{2} \operatorname{tr}\left(\mathbf{C}(\boldsymbol{\theta})^{-1} \frac{\partial \mathbf{C}(\boldsymbol{\theta})}{\partial \boldsymbol{\theta}} \mathbf{C}(\boldsymbol{\theta})^{-1} \frac{\partial \mathbf{C}(\boldsymbol{\theta})}{\partial \boldsymbol{\theta}}\right)+2 \mathfrak{R}\left[\frac{\partial \mathbf{x}(\boldsymbol{\theta})}{\partial \boldsymbol{\theta}} \mathbf{C}(\boldsymbol{\theta})^{-1} \frac{\partial \mathbf{x}(\boldsymbol{\theta})^{H}}{\partial \boldsymbol{\theta}}\right]
$$

where $\mathfrak{R}[\cdot]$ denotes the real part of the associated matrix.

\subsection{Summary}

In this chapter, the parameter estimation schemes considered in the development of the channel prediction scheme in this thesis are presented. After an introduction to the classical parameter estimation problem, the subspace based methods including MUSIC, ESPRIT, Root-MUSIC, Unitary-ESPRIT and Matrix Pencil were described. This is followed by a review of methods for subspace tracking and for estimation of parameters of linear models. Finally, a brief overview of the Cramer Rao lower bound for unbiased estimators was presented. 


\section{Part II}

\section{Original Contributions}





\section{4}

Narrowband MIMO Prediction

$1 \begin{gathered}\text { His chapter presents channel prediction schemes for narrowband MIMO } \\ \text { systems. The methods based on PRC modeling and multidimensional }\end{gathered}$ parameter estimation have been published in part in $[13,14]$.

\subsection{Prediction Models and Parametrization}

A commonly used multipath channel model is the ray based SOS model, defined for a SISO system as the superposition of $P$ scattering sources [93], that is

$$
h(t)=\sum_{p=1}^{P} \alpha_{p} e^{j \omega_{p} t}
$$

where $\alpha_{p}$ is the complex amplitude of the $p$ th scattering source and $\omega_{p}$ is the Doppler frequency. The SISO model in (4.1) can be extended to a MIMO propagation channel in $2 \mathrm{D}$ scenarios via the introduction of the transmit and 
receive spatial dimensions [189]

$$
\mathbf{H}(t)=\sum_{p=1}^{P} \alpha_{p} \mathbf{a}_{\mathbf{r}}\left(\theta_{p}\right) \mathbf{a}_{\mathrm{t}}^{T}\left(\phi_{p}\right) e^{j \omega_{p} t}
$$

where $\theta_{p}$ and $\phi_{p}$ are the AOA and AOD of the $p$ th path relative to the array broadsides, respectively, while $\mathbf{a}_{\mathrm{r}}\left(\theta_{p}\right)$ and $\mathbf{a}_{\mathrm{t}}\left(\phi_{p}\right)$ are the receive and transmit array response vectors in the direction of the $p$ th scattering source, respectively. It should be noted that (4.2) is applicable to any array geometry. Assuming that the receive antenna array is a ULA with $M$ equally spaced antenna elements along the $y$-axis at spatial locations

$$
\mathbf{r}_{m}=\left[\begin{array}{ll}
0 & m d_{\mathrm{r}}
\end{array}\right] ; \quad m=0,1, \ldots, M-1
$$

where $d_{\mathrm{r}}$ denotes the distance between adjacent antenna elements. Now assume that wave propagation is in the $x-y$ plane with wave vector

$$
\kappa_{p}=\frac{2 \pi}{\lambda}\left[\begin{array}{c}
\cos \theta_{p} \\
\sin \theta_{p}
\end{array}\right]
$$

The $m$ th element of the receive array response vector is thus

$$
\begin{aligned}
\mathbf{a}_{\mathrm{r}}^{m}\left(\theta_{p}\right) & =e^{-j \mathbf{r}_{m} \kappa_{p}} \\
& =e^{-j 2 \pi m d_{\mathrm{r}} \sin \theta_{p}}
\end{aligned}
$$

The $M \times 1$ array response vector is thus

$$
\mathbf{a}_{\mathrm{r}}\left(\theta_{p}\right)=\left[\begin{array}{c}
1 \\
e^{-j 2 \pi d_{\mathrm{r}} \sin \theta_{p}} \\
e^{-j 4 \pi d_{\mathrm{r}} \sin \theta_{p}} \\
\vdots \\
e^{-j 2(M-1) \pi d_{\mathrm{r}} \sin \theta_{p}}
\end{array}\right]
$$

Defining $\mu_{p}^{\mathrm{r}}=-2 \pi d_{\mathrm{r}} \sin \theta_{p},(4.6)$ becomes

$$
\mathbf{a}_{\mathrm{r}}\left(\mu_{p}^{\mathrm{r}}\right)=\left[1, e^{j \mu_{p}^{\mathrm{r}}}, \cdots, e^{j(M-1) \mu_{p}^{\mathrm{r}}}\right]^{T}
$$


Analogous to (4.7), the transmit array response vector, $\mathbf{a}_{\mathrm{t}}\left(\mu_{p}^{\mathrm{t}}\right)$ is defined as

$$
\mathbf{a}_{\mathrm{t}}\left(\mu_{p}^{\mathrm{t}}\right)=\left[1, e^{j \mu_{p}^{\mathrm{t}}}, \cdots, e^{j(N-1) \mu_{p}^{\mathrm{t}}}\right]^{T}
$$

where $\mu_{p}^{\mathrm{t}}=-2 \pi d_{\mathrm{t}} \sin \phi_{p}$. $d_{\mathrm{t}}$ denotes the antenna separation at the transmitter.

Assuming that the channel is sampled with period $T_{\text {samp }}$, the CSI at discrete time instant, $k$ is obtained from (4.2) as

$$
\mathbf{H}(k)=\sum_{p=1}^{P} \alpha_{p} \mathbf{a}_{\mathrm{r}}\left(\mu_{p}^{\mathrm{r}}\right) \mathbf{a}_{\mathrm{t}}^{T}\left(\mu_{p}^{\mathrm{t}}\right) e^{j k \nu_{p}}
$$

where $\nu_{p}=\omega_{p} T_{\text {samp }}$ is the normalized Doppler shift of the $p$-th path. As shown in (4.9), each path is characterized by the parameter set $\left\{\alpha_{p}, \mu_{p}^{\mathrm{r}}, \mu_{p}^{\mathrm{t}}, \nu_{p}\right\}^{1}$. In order to reduce the problem of modeling and extrapolating the time varying MIMO channel to a parameter estimation problem, we assume that the multipath parameters remain constant over the region for which channel observation/measurement and prediction is made. This assumption has been shown in the industry standard 3GPP/WINNER II SCM model [3, p. 55] to be valid for mobile movements between $10-50 \lambda$. The validity range is dependent on factors such as the carrier frequency, mobile velocity and distance between the mobile and the scattering sources. A rule-of-thumb for evaluating the validity range is [60]

$$
T_{\text {valid }}=\sqrt{\frac{c r_{\text {min }}}{3 f_{\mathrm{c}} v^{2}}}
$$

where $c=3.0 \times 10^{8}$ is the speed of light, $r_{\min }$ is the distance between the mobile station and the nearest scatterer, $f_{\mathrm{c}}$ and $v$ are the carrier frequency and mobile velocity, respectively.

We also assume that the mobile velocity remains constant such that constant temporal sampling corresponds to constant spatial sampling ${ }^{2}$.

\footnotetext{
${ }^{1}$ Note that the parameterization can alternatively be expressed in the set $\left\{\alpha_{p}, \theta_{p}, \phi_{p}, \nu_{p}\right\}$.

${ }^{2}$ This assumption will allow us to treat the variation of the channel as a pure spatial phenomena and obtain results that are independent of mobile velocity
} 
The different formulations of the double directional model (4.9) that are considered in the development of the prediction schemes in this chapter will be derived in the sequel. These formulations are obtained by progressively removing from (4.9) the dependence on the transmit and/or receive array structure and the structural parameters. The impact of mutual coupling on the array response vectors is not considered in this thesis.

\subsubsection{DOA/DOD Model}

The DOD/DOA model is based on the assumption that the array response vectors $\mathbf{a}_{\mathrm{r}}$ and $\mathbf{a}_{\mathrm{t}}$ are explicit functions of the AOA and AOD [110] as shown in (4.9). It should be noted that (4.9) is valid for any array geometry. We will consider MIMO channels having a ULA at both ends.

\subsubsection{Transmit Spatial Signature Model (TSSM)}

The DOD/DOA model depends on the specific array configuration and the AOA and AOD. Since the transmitter is often stationary in mobile wireless systems, estimation of the AOD may be difficult and possibly not required for accurate prediction of the channel. We therefore replace the product of the complex amplitude and the transmit array steering vector for each path in (4.9) by an unstructured Transmit Spatial Signature (TSS) vector, $\mathbf{s}_{p}^{\mathrm{t}}$. A similar model termed vector spatial signature (VSS) was used in [110] while deriving performance bounds. The model in (4.9) can now be expressed as

$$
\mathbf{H}(k)=\sum_{p=1}^{P} \mathbf{a}_{\mathrm{r}}\left(\mu_{p}^{\mathrm{r}}\right)\left(\mathbf{s}_{p}^{\mathrm{t}}\right)^{T} e^{j k \nu_{p}}
$$

where $\mathbf{s}_{p}^{\mathrm{t}} \in \mathbb{C}^{N \times 1}$ is the TSS for the $p$ th propagating path.

\subsubsection{Receive Spatial Signature Model (RSSM)}

Similar to the TSSM, we assume that the receive array steering vector is not an explicit function of the AOA and replace the product of the complex 
Table 4.1: Narrowband MIMO Prediction Models: Parametrization and Dependence on Number of Antennas

\begin{tabular}{lll} 
Model & Parameters & No. of Real Valued Parameters \\
\hline DOA DOD & $\left\{\mathfrak{R}\left(\alpha_{p}\right), \mathfrak{I}\left(\alpha_{p}\right), \mu_{p}^{\mathrm{r}}, \mu_{p}^{\mathrm{t}}, \nu_{p}\right\}_{p=1}^{P}$ & $5 P$ \\
TSSM & $\left\{\mu_{p}^{\mathrm{r}}, \nu_{p}, \mathfrak{R}\left(\mathbf{s}_{p}\right), \mathfrak{I}\left(\mathbf{s}_{p}\right)\right\}_{p=1}^{P}$ & $P(2 N+2)$ \\
RSSM & $\left\{\mu_{p}^{\mathrm{t}}, \nu_{p}, \mathfrak{R}\left(\mathbf{s}_{p}\right), \mathfrak{I}\left(\mathbf{s}_{p}\right)\right\}_{p=1}^{P}$ & $P(2 M+3)$ \\
MSSM & $\left\{\nu_{p}, \mathfrak{R}\left(\mathbf{S}_{p}\right), \mathfrak{I}\left(\mathbf{S}_{p}\right)\right\}_{p=1}^{P}$ & $P(N M+1)$ \\
\hline
\end{tabular}

amplitude and receive array steering vector with an unstructured vector, $\mathbf{s}_{p}^{\mathrm{r}}$, giving

$$
\mathbf{H}(k)=\sum_{p=1}^{P} \mathbf{s}_{p}^{\mathrm{r}} \mathbf{a}_{\mathrm{t}}^{T}\left(\mu_{p}^{\mathrm{t}}\right) e^{j k \nu_{p}}
$$

where $\mathbf{s}^{\mathrm{r}} \in \mathbb{C}^{M \times 1}$ is the receive spatial signature (RSS) for the $p$ th path.

\subsubsection{Matrix Spatial Signature Model (MSSM)}

The Matrix Spatial Signature Model (MSSM) [110] replaces the product of both array steering vectors and the complex amplitude by an $M \times N$ unstructured matrix spatial signature, $\mathbf{S}$, giving

$$
\mathbf{H}(k)=\sum_{p=1}^{P} \mathbf{S}_{p} e^{j k \nu_{p}}
$$

Note that although all of the models described in this section are derived from the double directional MIMO model,(4.9), they differ in the parametrization and number of parameters required. A summary of the number of parameters required for each model and the dependence on the number of antenna elements is shown in Table 4.1. 


\subsection{Data Transformation}

In Section 4.1, we described different formulations of the double directional spatial channel model considered in the development of the prediction schemes in this chapter. We now present data preprocessing necessary for the extraction of the parameters of each of the models from the available channel observations. We assume that $K$ samples of the channel are available either from channel estimation or measurement. In practice, the estimated or measured channel will contain some imperfections resulting from noise and interference effects. The estimated CSI is therefore defined as

$$
\hat{\mathbf{H}}(k)=\mathbf{H}(k)+\mathbf{W}(k) ; \quad k=0,1, \cdots, K-1
$$

where $\mathbf{W}(k) \in \mathcal{C}^{M \times N}$ is a complex Gaussian random variable with zero mean and variance, $\sigma^{2}$, that accounts for the effects of noise and interference.

\subsubsection{DOA/DOD Transformation}

As shown in (4.9), the DOD/DOA model is characterized by $3 P$ structural parameters, $\left\{\mu_{p}^{\mathrm{r}}, \mu_{p}^{\mathrm{t}}, \nu_{p}\right\}_{p=1}^{P}$ and $P$ complex weight parameters corresponding to $2 P$ real valued weight parameters. Extraction of these parameters from the $K$ channel observations require a three dimensional array data structure. Since we consider MIMO systems with a 1-D array (i.e., a ULA) at both ends of the link, it is necessary to convert the data into a form that enables 3D parameter estimation. Let

$$
\begin{aligned}
\hat{\mathbf{h}} & =\operatorname{vec}[\hat{\mathbf{H}}(k)] \\
& =\left[\hat{h}_{11}(k), \hat{h}_{12}(k), \cdots, \hat{h}_{1 N}(k), \hat{h}_{21}(k), \cdots, \hat{h}_{2 N}(k), \cdots, \hat{h}_{M N}(k)\right]^{T}
\end{aligned}
$$


be a $N M \times 1$ be a vector obtained by stacking the columns of $\hat{\mathbf{H}}(k)$. Using (4.9), $\hat{\mathbf{h}}(k)$ can be expressed as

$$
\hat{\mathbf{h}}(k)=\left[\begin{array}{c}
\sum_{p=1}^{P} \alpha_{p} a_{\mathrm{r}, 1}\left(\mu_{p}^{\mathrm{r}}\right) a_{\mathrm{t}, 1}\left(\mu_{p}^{\mathrm{t}}\right) e^{j k \nu_{p}} \\
\sum_{p=1}^{P} \alpha_{p} a_{\mathrm{r}, 1}\left(\mu_{p}^{\mathrm{r}}\right) a_{\mathrm{t}, 2}\left(\mu_{p}^{\mathrm{t}}\right) e^{j k \nu_{p}} \\
\vdots \\
\sum_{p=1}^{P} \alpha_{p} a_{\mathrm{r}, 1}\left(\mu_{p}^{\mathrm{r}}\right) a_{\mathrm{t}, N}\left(\mu_{p}^{\mathrm{t}}\right) e^{j k \nu_{p}} \\
\sum_{p=1}^{P} \alpha_{p} a_{\mathrm{r}, 2}\left(\mu_{p}^{\mathrm{r}}\right) a_{\mathrm{t}, 1}\left(\mu_{p}^{\mathrm{t}}\right) e^{j k \nu_{p}} \\
\vdots \\
\sum_{p=1}^{P} \alpha_{p} a_{\mathrm{r}, M}\left(\mu_{p}^{\mathrm{r}}\right) a_{\mathrm{t}, N}\left(\mu_{p}^{\mathrm{t}}\right) e^{j k \nu_{p}}
\end{array}\right]+\mathbf{w}(k)
$$

which in turn, using the properties of the Kronecker product ${ }^{3}$, can be written as

$$
\hat{\mathbf{h}}(k)=\sum_{p=1}^{P} \alpha_{p}\left(\mathbf{a}_{\mathrm{r}}\left(\mu_{p}^{\mathrm{r}}\right) \otimes \mathbf{a}_{\mathrm{t}}\left(\mu_{p}^{\mathrm{t}}\right)\right) e^{j k \nu_{p}}+\mathbf{w}(k)
$$

The transmit and receive spatial dimensions of the channel are combined by virtue of the Kronecker product in (4.17). It should be noted that the structure of (4.17) allows for the estimation of the AOA and AOD, and similar vectorized channel have been used in $[115,131]$ to jointly estimating these parameters. However, the number of paths that can be resolved by this formulation is limited by the total number of antennas at the transmit and receive end making its application to practical MIMO systems with small numbers of antennas unattractive. Moreover, these approaches suffer from the left/right ambiguity problem. We overcome these limitations in our methods by parameterizing the channel in terms of the direction cosines/spatial frequencies and additionally introduce the temporal dimension into (4.17).

\footnotetext{
${ }^{3}$ The Kronecker product of a $N \times M$ matrix $\mathbf{A}$ and a $P \times Q$ matrix $\mathbf{B}$ is the $N P \times M Q$ matrix defined as

$$
\mathbf{A} \otimes \mathbf{B}=\left[\begin{array}{cccc}
a_{1,1} \mathbf{B} & a_{1,2} \mathbf{B} & \cdots & a_{1, M} \mathbf{B} \\
a_{2,1} \mathbf{B} & a_{2,2} \mathbf{B} & \cdots & a_{2, M} \mathbf{B} \\
\vdots & \vdots & \ddots & \vdots \\
a_{N, 1} \mathbf{B} & a_{N, 2} \mathbf{B} & \cdots & a_{N, M} \mathbf{B}
\end{array}\right]
$$
}


We form the Hankel matrix

$$
\hat{\mathcal{H}}_{\mathrm{d}}=\left[\begin{array}{cccc}
\hat{\mathbf{h}}(0) & \hat{\mathbf{h}}(1) & \cdots & \hat{\mathbf{h}}\left(T_{\mathrm{d}}\right) \\
\hat{\mathbf{h}}(1) & \hat{\mathbf{h}}(2) & \cdots & \hat{\mathbf{h}}\left(T_{\mathrm{d}}+1\right) \\
\vdots & \vdots & \ddots & \vdots \\
\hat{\mathbf{h}}\left(R_{\mathrm{d}}\right) & \hat{\mathbf{h}}\left(R_{\mathrm{d}}+1\right) & \cdots & \hat{\mathbf{h}}(K-1)
\end{array}\right]
$$

where $T_{\mathrm{d}}$ and $R_{\mathrm{d}}=K-T_{\mathrm{d}}$ are the Hankel matrix parameters selected such that $N M R_{\mathrm{d}} \leq P_{\max }+1$. We note that there is a compromise in selecting these free parameters. Large values of $R_{\mathrm{d}}$ increases the number of rows in (4.18) and hence the number of paths that can be resolved, but this results in small values of $T_{\mathrm{d}}$ which affects the accuracy of covariance estimates. Note that each column of $\hat{\mathcal{H}}_{\mathrm{d}}$ corresponds to a stack of $R_{\mathrm{d}}$ vectorized observations in (4.16) and can be expressed as

$$
\hat{\mathcal{H}}_{\mathrm{d}}(:, a)=\sum_{p=1}^{P}\left\{\alpha_{p}\left[\begin{array}{c}
\mathbf{a}_{\mathrm{r}}\left(\mu_{p}^{\mathrm{r}}\right) \otimes \mathbf{a}_{\mathrm{t}}\left(\mu_{p}^{\mathrm{t}}\right) \\
\left(\mathbf{a}_{\mathrm{r}}\left(\mu_{p}^{\mathrm{r}}\right) \otimes \mathbf{a}_{\mathrm{t}}\left(\mu_{p}^{\mathrm{t}}\right)\right) e^{j \nu_{p}} \\
\left(\mathbf{a}_{\mathrm{r}}\left(\mu_{p}^{\mathrm{r}}\right) \otimes \mathbf{a}_{\mathrm{t}}\left(\mu_{p}^{\mathrm{t}}\right)\right) e^{j 2 \nu_{p}} \\
\vdots \\
\left(\mathbf{a}_{\mathrm{r}}\left(\mu_{p}^{\mathrm{r}}\right) \otimes \mathbf{a}_{\mathrm{t}}\left(\mu_{p}^{\mathrm{t}}\right)\right) e^{j\left(R_{\mathrm{d}}-1\right) \nu_{p}}
\end{array}\right] e^{j(a-1) \nu_{p}}\right\}+\mathbf{w}(a)
$$

for $a=1,2, \cdots, T_{\mathrm{d}}$. Again, the relationship between vectorization and Kronecker products simplifies (4.19) to

$$
\hat{\mathcal{H}}_{\mathrm{d}}(:, a)=\sum_{p=1}^{P} \alpha_{p} \mathbf{a}\left(\mu_{p}^{\mathrm{r}}, \mu_{p}^{\mathrm{t}}, \nu_{p}\right) e^{j(a-1) \nu_{p}}+\mathbf{w}(a)
$$

where

$$
\mathbf{a}\left(\mu_{p}^{\mathrm{r}}, \mu_{p}^{\mathrm{t}}, \nu_{p}\right)=\mathbf{a}_{\mathrm{r}}\left(\mu_{p}^{\mathrm{r}}\right) \otimes \mathbf{a}_{\mathrm{t}}\left(\mu_{p}^{\mathrm{t}}\right) \otimes \mathbf{a}_{\mathrm{d}}\left(\nu_{p}\right)
$$

$\mathbf{a}_{\mathrm{d}}\left(\nu_{p}\right)$ is the $R_{\mathrm{d}} \times 1$ vector $^{4}$

$$
\mathbf{a}_{\mathrm{d}}\left(\nu_{p}\right)=\left[\begin{array}{lllll}
1 & e^{j \nu_{p}} & e^{j 2 \nu_{p}} & \cdots & e^{j\left(R_{\mathrm{d}}-1\right) \nu_{p}}
\end{array}\right]^{T}
$$

\footnotetext{
${ }^{4}$ This corresponds to the array response vector of a ULA in the direction of motion of the mobile station. The transformed data in (4.19) can therefore be interpreted as $T_{\mathrm{d}}$ observations from a $M \times N \times R_{\mathrm{d}}$ array.
} 
Defining $\beta_{p}(a)=\alpha_{p} e^{j(a-1) \nu_{p}},(4.20)$ can be written as

$$
\begin{aligned}
\hat{\mathcal{H}}_{\mathrm{d}}(:, a) & =\sum_{p=1}^{P} \beta_{p}(a) \mathbf{a}\left(\mu_{p}^{\mathrm{r}}, \mu_{p}^{\mathrm{t}}, \nu_{p}\right)+\mathbf{w}(a) \\
& =\mathbf{A}\left(\boldsymbol{\mu}^{\mathrm{r}}, \boldsymbol{\mu}^{\mathrm{t}}, \boldsymbol{\nu}\right) \boldsymbol{\beta}(a)+\mathbf{w}(a)
\end{aligned}
$$

where

$$
\boldsymbol{\beta}(a)=\left[\begin{array}{llll}
\beta_{1}(a) & \beta_{2}(a) & \cdots & \beta_{P}(a)
\end{array}\right]^{T}
$$

and $\mathbf{A}\left(\boldsymbol{\mu}^{\mathrm{r}}, \boldsymbol{\mu}^{\mathrm{t}}, \boldsymbol{\nu}\right)=\left[\begin{array}{lll}\mathbf{a}\left(\mu_{1}^{\mathrm{r}}, \mu_{1}^{\mathrm{t}}, \nu_{1}\right) & \cdots & \mathbf{a}\left(\mu_{P}^{\mathrm{r}}, \mu_{P}^{\mathrm{t}}, \nu_{P}\right)\end{array}\right]$ is a Vandermonde structured response matrix which can be expressed as a product of three Vandermonde matrices ${ }^{5}$

$$
\mathbf{A}=\mathbf{A}_{\mathrm{r}} \diamond \mathbf{A}_{\mathrm{t}} \diamond \mathbf{A}_{\mathrm{d}}
$$

where $\diamond$ denotes the Khatri-Rao product ${ }^{6}$,

$$
\begin{aligned}
\mathbf{A}_{\mathrm{r}} & =\left[\begin{array}{cccc}
1 & 1 & \cdots & 1 \\
e^{j \mu_{1}^{\mathrm{r}}} & e^{j \mu_{2}^{\mathrm{r}}} & \cdots & e^{j \mu_{P}^{\mathrm{r}}} \\
\vdots & \vdots & \ddots & \vdots \\
e^{j(M-1) \mu_{1}^{\mathrm{r}}} & e^{j(M-1) \mu_{2}^{\mathrm{r}}} & \cdots & e^{j(M-1) \mu_{P}^{\mathrm{r}}}
\end{array}\right] \\
\mathbf{A}_{\mathrm{t}} & {\left[\begin{array}{cccc}
1 & 1 & \cdots & 1 \\
e^{j \mu_{1}^{\mathrm{t}}} & e^{j \mu_{2}^{\mathrm{t}}} & \cdots & e^{j \mu_{P}^{\mathrm{t}}} \\
\vdots & \vdots & \ddots & \vdots \\
e^{j(N-1) \mu_{1}^{\mathrm{t}}} & e^{j(N-1) \mu_{2}^{\mathrm{t}}} & \cdots & e^{j(N-1) \mu_{P}^{\mathrm{t}}}
\end{array}\right] }
\end{aligned}
$$

and

$$
\mathbf{A}_{\mathrm{d}}=\left[\begin{array}{cccc}
1 & 1 & \cdots & 1 \\
e^{j \nu_{1}} & e^{j \nu_{2}} & \cdots & e^{j \nu_{P}} \\
\vdots & \vdots & \ddots & \vdots \\
e^{j\left(R_{\mathrm{d}}-1\right) \nu_{1}} & e^{j\left(R_{\mathrm{d}}-1\right) \nu_{2}} & \cdots & e^{j\left(R_{\mathrm{d}}-1\right) \nu_{P}}
\end{array}\right]
$$

\footnotetext{
${ }^{5}$ The dependence of the matrices on the channel parameters have been omitted here for simplicity.

${ }^{6}$ The Khatrio-Rao product of two matrices $\mathbf{A}=\left[\begin{array}{llll}\mathbf{a}_{1} & \mathbf{a}_{2} & \cdots & \mathbf{a}_{P}\end{array}\right]$ and $\mathbf{B}=$ $\left[\begin{array}{llll}\mathbf{b}_{1} & \mathbf{b}_{2} & \cdots & \mathbf{b}_{P}\end{array}\right]$ is defined as the column-wise Kronecker product of these matrices, thus $\mathbf{A} \diamond \mathbf{B}=\left[\begin{array}{llll}\mathbf{a}_{1} \otimes \mathbf{b}_{1} & \mathbf{a}_{2} \otimes \mathbf{b}_{2} & \cdots & \mathbf{a}_{P} \otimes \mathbf{b}_{P}\end{array}\right]$.
} 
Table 4.2: Narrowband Transformed MIMO Data Domains and Dependence on Channel Parameters

\begin{tabular}{llll}
\hline \multirow{2}{*}{ Model } & \multicolumn{3}{c}{ Data Domain } \\
\cline { 2 - 4 } & Receive Spatial & Transmit Spatial & Temporal \\
\hline DOA/DOD & AOA & AOD & Doppler shift \\
TSSM & AOA & - & Doppler shift \\
RSSM & - & AOD & Doppler shift \\
MSSM & - & - & Doppler shift \\
\hline
\end{tabular}

Clearly, (4.23) corresponds to a three dimensional data model obtained by combining the transmit spatial, receive spatial and temporal dimensions of the narrowband MIMO channel.

\subsubsection{TSSM Transformation}

As shown in (4.10) and Table 4.1, the Transmit Spatial Signature Model (TSSM) is parametrized by $2 P$ structural parameters $\left\{\mu_{p}^{\mathrm{r}}, \nu_{p}\right\}_{p=1}^{P}$. We will here describe the transformation required for the joint extraction of these parameters from (4.10). Using the $K$ CSI samples, we form the $M R_{\mathrm{t}} \times N T_{\mathrm{t}}$ Hankel matrix

$$
\hat{\mathcal{H}}_{\mathrm{t}}=\left[\begin{array}{cccc}
\hat{\mathbf{H}}(0) & \hat{\mathbf{H}}(1) & \cdots & \hat{\mathbf{H}}\left(T_{\mathrm{t}}\right) \\
\hat{\mathbf{H}}(1) & \hat{\mathbf{H}}(2) & \cdots & \hat{\mathbf{H}}\left(T_{\mathrm{t}}+1\right) \\
\vdots & \vdots & \ddots & \vdots \\
\hat{\mathbf{H}}\left(R_{\mathrm{t}}\right) & \hat{\mathbf{H}}\left(R_{\mathrm{t}}+1\right) & \cdots & \hat{\mathbf{H}}(K-1)
\end{array}\right]
$$


Using (4.10) and the transformation in (4.29), each column of (4.29) can be modeled as

$$
\hat{\mathcal{H}}_{\mathrm{t}}(:, a)=\sum_{p=1}^{P}\left\{\left[\begin{array}{c}
\mathbf{a}_{\mathrm{r}}\left(\mu_{p}^{\mathrm{r}}\right) \\
\mathbf{a}_{\mathrm{r}}\left(\mu_{p}^{\mathrm{r}}\right) e^{j \nu_{p}} \\
\mathbf{a}_{\mathrm{r}}\left(\mu_{p}^{\mathrm{r}}\right) e^{j 2 \nu_{p}} \\
\vdots \\
\mathbf{a}_{\mathrm{r}}\left(\mu_{p}^{\mathrm{r}}\right) e^{j\left(R_{\mathrm{t}}-1\right) \nu_{p}}
\end{array}\right] s_{a} e^{j(a-1) \nu_{p}}\right\}+\mathbf{w}(a)
$$

where

$$
s_{a}= \begin{cases}\mathbf{s}^{\mathrm{t}}(a) & : 1 \leq a \leq M \\ \mathbf{s}^{\mathrm{t}}(a-M) & : M+1 \leq a \leq 2 M \\ \vdots & \vdots \\ \mathbf{s}^{\mathrm{t}}\left(a-\left(T_{\mathrm{t}}-1\right) M\right) & :\left(T_{\mathrm{t}}-1\right) M+1 \leq a \leq T_{\mathrm{t}} M\end{cases}
$$

with $\mathbf{s}^{\mathrm{t}}(i)$ denoting the $i$ th entry of $\mathbf{s}^{\mathrm{t}}$. The Kronecker product can again be utilized to simplify (4.30)

$$
\begin{aligned}
\hat{\mathcal{H}}_{\mathrm{t}}(:, a) & =\sum_{p=1}^{P}\left(\mathbf{a}_{\mathrm{r}}\left(\mu_{p}^{\mathrm{r}}\right) \otimes \mathbf{a}_{\mathrm{d}}\left(\nu_{p}\right)\right) s_{a} e^{j(a-1) \nu_{p}}+\mathbf{w}(a) \\
& =\mathbf{A}\left(\boldsymbol{\mu}^{\mathrm{r}}, \boldsymbol{\nu}\right) \boldsymbol{\beta}_{a}+\mathbf{w}(a)
\end{aligned}
$$

Here, $\boldsymbol{\beta}_{a}=\left[\begin{array}{lll}s_{a} e^{j(a-1) \nu_{1}} & \cdots & s_{a} e^{j(a-1) \nu_{P}}\end{array}\right]^{T}$ and $\mathbf{A}\left(\boldsymbol{\mu}^{\mathrm{r}}, \boldsymbol{\nu}\right)$ is defined as

$$
\mathbf{A}\left(\boldsymbol{\mu}^{\mathrm{r}}, \boldsymbol{\nu}\right)=\mathbf{A}_{\mathrm{r}}\left(\boldsymbol{\mu}^{\mathrm{r}}\right) \diamond \mathbf{A}_{\mathrm{d}}(\boldsymbol{\nu})
$$

where $\mathbf{A}_{\mathrm{r}}$ and $\mathbf{A}_{\mathrm{d}}$ are given in (4.26) and (4.28), respectively. As illustrated in the transformed TSSM in (4.32), the array response vector represents a two dimensional data obtained by combining the receive spatial and temporal dimensions of the MIMO channel.

\subsubsection{RSSM Transformation}

Similar to the TSSM, parameterizing the Receive Spatial Signature Model (RSSM) requires $2 P$ structural parameters $\left\{\mu_{p}^{\mathrm{t}}, \nu_{p}\right\}_{p=1}^{P}$. We here form the 
$N R_{\mathrm{r}} \times M T_{\mathrm{r}}$ Hankel matrix

$$
\hat{\mathcal{H}}_{\mathrm{r}}=\left[\begin{array}{cccc}
\hat{\mathbf{H}}^{T}(0) & \hat{\mathbf{H}}^{T}(1) & \cdots & \hat{\mathbf{H}}^{T}\left(T_{\mathrm{r}}\right) \\
\hat{\mathbf{H}}^{T}(1) & \hat{\mathbf{H}}^{T}(2) & \cdots & \hat{\mathbf{H}}^{T}\left(T_{\mathrm{r}}+1\right) \\
\vdots & \vdots & \ddots & \vdots \\
\hat{\mathbf{H}}^{T}\left(R_{\mathrm{r}}\right) & \hat{\mathbf{H}}^{T}\left(R_{\mathrm{r}}+1\right) & \cdots & \hat{\mathbf{H}}^{T}(K-1)
\end{array}\right]
$$

using the $K$ available noisy data. Using (4.11), the columns of (4.34) can be shown to be

$$
\hat{\mathcal{H}}_{\mathrm{r}}(:, b)=\sum_{p=1}^{P}\left\{\left[\begin{array}{c}
\mathbf{a}_{\mathrm{t}}\left(\mu_{p}^{\mathrm{t}}\right) \\
\mathbf{a}_{\mathrm{t}}\left(\mu_{p}^{\mathrm{t}}\right) e^{j \nu_{p}} \\
\mathbf{a}_{\mathrm{t}}\left(\mu_{p}^{\mathrm{t}}\right) e^{j 2 \nu_{p}} \\
\vdots \\
\mathbf{a}_{\mathrm{t}}\left(\mu_{p}^{\mathrm{t}}\right) e^{j\left(R_{\mathrm{t}}-1\right) \nu_{p}}
\end{array}\right] s_{b} e^{j(b-1) \nu_{p}}\right\}+\mathbf{w}(b)
$$

for $a=1,2, \cdots, T_{\mathrm{r}}$. Using the structure of $(4.34), s_{b}$ can be expressed as

$$
s_{b}= \begin{cases}\mathbf{s}^{\mathrm{r}}(b) & : 1 \leq b \leq N \\ \mathbf{s}^{\mathrm{r}}(b-M) & : N+1 \leq b \leq 2 N \\ \vdots & \vdots \\ \mathbf{s}^{\mathrm{r}}\left(b-\left(T_{\mathrm{r}}-1\right) N\right) & :\left(T_{\mathrm{r}}-1\right) N+1 \leq b \leq T_{\mathrm{r}} N\end{cases}
$$

Similar to (4.32), (4.35) can be written as

$$
\begin{aligned}
\hat{\mathcal{H}}_{\mathrm{r}}(:, b) & =\sum_{p=1}^{P}\left(\mathbf{a}_{\mathrm{t}}\left(\mu_{p}^{\mathrm{t}}\right) \otimes \mathbf{a}_{\mathrm{d}}\left(\nu_{p}\right)\right) s_{b} e^{j(b-1) \nu_{p}}+\mathbf{w}(b) \\
& =\mathbf{A}\left(\boldsymbol{\mu}^{\mathrm{t}}, \boldsymbol{\nu}\right) \boldsymbol{\beta}_{b}+\mathbf{w}(b)
\end{aligned}
$$

with $\boldsymbol{\beta}_{b}=\left[\begin{array}{lll}s_{b} e^{j(b-1) \nu_{1}} & \cdots & s_{b} e^{j(b-1) \nu_{P}}\end{array}\right]^{T}$ and $\mathbf{A}\left(\boldsymbol{\mu}^{\mathrm{t}}, \boldsymbol{\nu}\right)$ is defined analogously to (4.33). It should be noted that the TSSM and RSSM have a number of similarities including the number of structural parameters and the transformation procedure. However, while the former depends on the receive correlation, the latter utilizes the transmit spatial structure of the channel. Moreover, the performance and application of these methods is dependent 
on the number of transmit and receive antenna elements. For instance, while the TSSM is applicable to Single-input multiple-output (SIMO) channels, the RSSM is suitable for Multiple-input single-output (MISO) channels. Furthermore, the choice of either of these methods in MIMO systems depends on the number of antenna elements at either end of the link.

\subsubsection{MSSM Transformation}

The MSSM is parameterized by $P$ Doppler shifts $\left\{\nu_{p}\right\}_{p=1}^{P}$ which can be extracted from the temporal properties of the channel. Consider the vectorized channel in (4.14) and define the $R_{\mathrm{m}} \times N M T_{\mathrm{m}}$ Hankel matrix

$$
\hat{\mathcal{H}}_{\mathrm{m}}=\left[\begin{array}{cccc}
\hat{\mathbf{h}}^{T}(0) & \hat{\mathbf{h}}^{T}(1) & \cdots & \hat{\mathbf{h}}^{T}\left(T_{\mathrm{m}}\right) \\
\hat{\mathbf{h}}^{T}(1) & \hat{\mathbf{h}}^{T}(2) & \cdots & \hat{\mathbf{h}}^{T}\left(T_{\mathrm{m}}+1\right) \\
\vdots & \vdots & \ddots & \vdots \\
\hat{\mathbf{h}}^{T}\left(R_{\mathrm{m}}\right) & \hat{\mathbf{h}}^{T}\left(R_{\mathrm{m}}+1\right) & \cdots & \hat{\mathbf{h}}^{T}(K-1)
\end{array}\right]
$$

where $R_{\mathrm{m}}$ and $T_{\mathrm{m}}=K-R_{\mathrm{m}}+1$ are the Hankel matrix size parameters, chosen such that $R_{\mathrm{m}} \geq P_{\max }+1$. Note that each column of (4.38) corresponds to $R_{\mathrm{m}}$ temporal measurements of the channel and can be modeled as

$$
\begin{aligned}
\hat{\mathcal{H}}_{\mathrm{m}}(:, c) & =\sum_{p=1}^{P} \beta_{p}(c) \mathbf{a}_{\mathrm{d}}\left(\nu_{p}\right)+\mathbf{w}(c) \\
& =\mathbf{A}(\boldsymbol{\nu}) \boldsymbol{\beta}+\mathbf{w}(c)
\end{aligned}
$$

where $\mathbf{A}=\mathbf{A}_{\mathrm{d}}$ is the Vandermonde matrix in (4.28) with $R_{\mathrm{d}}$ replaced with $R_{\mathrm{m}}$ and $\beta_{p}(c)$ is defined as

$$
\boldsymbol{\beta}(c: c+N M)=\left\{\left[\begin{array}{c}
\alpha_{p} a_{\mathrm{r}, 1}\left(\mu_{p}^{\mathrm{r}}\right) a_{\mathrm{t}, 1}\left(\mu_{p}^{\mathrm{t}}\right) \\
\alpha_{p} a_{\mathrm{r}, 1}\left(\mu_{p}^{\mathrm{r}}\right) a_{\mathrm{t}, 2}\left(\mu_{p}^{\mathrm{t}}\right) \\
\vdots \\
\alpha_{p} a_{\mathrm{r}, 1}\left(\mu_{p}^{\mathrm{r}}\right) a_{\mathrm{t}, N}\left(\mu_{p}^{\mathrm{t}}\right) \\
\alpha_{p} a_{\mathrm{r}, 2}\left(\mu_{p}^{\mathrm{r}}\right) a_{\mathrm{t}, 1}\left(\mu_{p}^{\mathrm{t}}\right) \\
\vdots \\
\alpha_{p} a_{\mathrm{r}, M}\left(\mu_{p}^{\mathrm{r}}\right) a_{\mathrm{t}, N}\left(\mu_{p}^{\mathrm{t}}\right)
\end{array}\right] \diamond\left[\begin{array}{c}
e^{j(c-1) \nu_{p}} \\
e^{j c \nu_{p}} \\
\vdots \\
e^{j(c+1) \nu_{p}} \\
e^{j(c+2) \nu_{p}} \\
\vdots \\
e^{j(c+N M-1)}
\end{array}\right]\right\}
$$


for $c=1: N M: N M T_{\mathrm{m}}$. A summary of the dimensions in the transformed data along with dependence on channel parameters is shown in Table 4.2.

\subsection{Translational Invariance in Multidimensional Vandermonde Matrices}

In the previous sections, we introduced the channel models that are utilized for the development of our prediction algorithms as well as the required data transformations. As shown in (4.23),(4.32), (4.37) and (4.39), the transformed data has response matrices which are Khatri-Rao products of Vandermonde matrices. The structure of the transformed data can therefore be utilized to jointly estimate the parameters of the channel. This can be achieved via multidimensional extensions of the ESPRIT [126, 159] scheme described in Section 3.2.3. Our choice of ESPRIT based methods is motivated by its super-resolution and relative simplicity over other parameter estimation methods. Moreover, since our transformed data exhibit some invariance structure in all dimensions, it appears natural to pursue methods which apply these invariance property for direct estimation of the desired parameters. An alternative and possibly better approach to estimate the multiple parameters from the transformed data is to use tensor-based ESPRIT schemes [75, 157]. The tensor based approach is not used in this thesis for a number of reasons. The tensor based schemes offer improved performance compared to the standard ESPRIT method only if the number of paths is strictly less than the number of antennas in at least one of the dimensions in the data [75]. For practical MIMO systems, there is no guarantee that this requirement will be satisfied in any propagation environment. Moreover, the high order SVD (HOSVD) upon which the tensor schemes relies for subspace estimation result in a much higher computational complexity.

In this section, we introduce the concept of translational invariance in multidimensional Vandermonde matrices that will form the basis for the pa- 
rameter estimation stage of the proposed scheme and derive expressions for selection operators that are required in the implementation of the proposed schemes. Consider a multi- dimensional Vandermonde matrix defined as the Khatri-Rao product of one-dimensional Vandermonde matrices thus

$$
\mathbf{A}=\mathbf{A}_{\mathrm{a}} \diamond \mathbf{A}_{\mathrm{b}} \diamond \mathbf{A}_{\mathrm{c}} \diamond \cdots \diamond \mathbf{A}_{\mathrm{u}}
$$

Assuming that $\mathbf{A}_{v} ; v \in[\mathrm{a}, \mathrm{b}, \cdots, \mathrm{u}]$ has dimension $L_{v} \times P, \mathbf{A}$ has dimension $L \times P$ with

$$
L=\prod_{v} L_{v}
$$

Let $\mathbf{A}_{v} ; v \in[\mathrm{a}, \mathrm{b}, \cdots, \mathrm{u}]$ be defined as

$$
\mathbf{A}_{v}=\left[\begin{array}{cccc}
1 & 1 & \cdots & 1 \\
v_{1} & v_{2} & \cdots & v_{P} \\
\vdots & \vdots & \ddots & \vdots \\
v_{1}^{\left(L_{\mathrm{v}}-1\right)} & v_{2}^{\left(L_{\mathrm{v}}-1\right)} & \cdots & v_{P}^{\left(L_{\mathrm{v}}-1\right)}
\end{array}\right]
$$

Our aim is to form two sub-matrices for each dimension from (4.41) exhibiting translational invariance similar to that in (3.19). Let $\mathbf{A}_{\mathrm{a} 1}$ and $\mathbf{A}_{\mathrm{a} 2}$ be submatrices obtained from (4.41) as follows

$$
\begin{aligned}
& \mathbf{A}_{\mathrm{a} 1}=\mathbf{A}_{\mathrm{a}}^{\mathrm{up}} \diamond \mathbf{A}_{\mathrm{b}} \diamond \mathbf{A}_{\mathrm{c}} \diamond \cdots \diamond \mathbf{A}_{\mathrm{u}} \\
& \mathbf{A}_{\mathrm{a} 2}=\mathbf{A}_{\mathrm{a}}^{\text {down }} \diamond \mathbf{A}_{\mathrm{b}} \diamond \mathbf{A}_{\mathrm{c}} \diamond \cdots \diamond \mathbf{A}_{\mathrm{u}}
\end{aligned}
$$

where $\mathbf{A}_{\mathrm{a}}^{\mathrm{up}}$ and $\mathbf{A}_{\mathrm{a}}^{\text {down }}$ are submatrices selected from $\mathbf{A}_{\mathrm{a}}$ by deleting the first and last rows, respectively. It is straightforward to show that $\mathbf{A}_{\mathrm{a} 1}$ and $\mathbf{A}_{\mathrm{a} 2}$ satisfy the invariance equation

$$
\mathbf{A}_{\mathrm{a} 2}=\mathbf{A}_{\mathrm{a} 1} \mathbf{D}(\mathbf{a})
$$

where $\mathbf{D}(\mathbf{a})$ is the diagonal matrix

$$
\mathbf{D}(\mathbf{a})=\left[\begin{array}{cccc}
a_{1} & 0 & \cdots & 0 \\
0 & a_{2} & \cdots & 0 \\
\vdots & \vdots & \ddots & \vdots \\
0 & 0 & \cdots & a_{P}
\end{array}\right]
$$


Assuming that $\mathbf{A}_{\mathrm{a}}$ is known, (4.45) can be solved for $\left\{a_{p}\right\}_{p=1}^{P}$. Note that $\mathbf{A}_{\mathrm{a}}^{\text {up }}$ and $\mathbf{A}_{\mathrm{a}}^{\text {down }}$ can be obtained from $\mathbf{A}_{\mathrm{a}}$ by multiplying it with the selection matrices

$$
\begin{aligned}
& \mathbf{J}_{\mathrm{a} 1}=\left[\begin{array}{ll}
\mathbf{I}_{L_{\mathrm{a}}-1} & \mathbf{0}_{L_{\mathrm{a}}-1}
\end{array}\right] \\
& \mathbf{J}_{\mathrm{a} 2}=\left[\begin{array}{ll}
\mathbf{0}_{L_{\mathrm{a}}-1} & \mathbf{I}_{L_{\mathrm{a}}-1}
\end{array}\right]
\end{aligned}
$$

For example, with $L_{\mathrm{a}}=4$, the selection matrices in (4.47) become

$$
\mathbf{J}_{\mathrm{a} 1}=\left[\begin{array}{llll}
1 & 0 & 0 & 0 \\
0 & 1 & 0 & 0 \\
0 & 0 & 1 & 0
\end{array}\right]
$$

and

$$
\mathbf{J}_{\mathrm{a} 2}=\left[\begin{array}{llll}
0 & 1 & 0 & 0 \\
0 & 0 & 1 & 0 \\
0 & 0 & 0 & 1
\end{array}\right]
$$

Substituting (4.47) into (4.44) and applying the identity $\mathbf{A}=$ IA yields

$$
\begin{aligned}
& \mathbf{A}_{\mathrm{a} 1}=\left[\begin{array}{ll}
\mathbf{I}_{L_{\mathrm{a}}-1} & \mathbf{0}_{L_{\mathrm{a}}-1}
\end{array}\right] \mathbf{A}_{\mathrm{a}} \diamond \mathbf{I}_{L_{\mathrm{b}}} \mathbf{A}_{\mathrm{b}} \diamond \mathbf{I}_{L_{\mathrm{c}}} \mathbf{A}_{\mathrm{c}} \diamond \cdots \diamond \mathbf{I}_{L_{\mathrm{u}}} \mathbf{A}_{\mathrm{u}} \\
& \mathbf{A}_{\mathrm{a} 2}=\left[\begin{array}{ll}
\mathbf{0}_{L_{\mathrm{a}}-1} & \mathbf{I}_{L_{\mathrm{a}}-1}
\end{array}\right] \mathbf{A}_{\mathrm{a}} \diamond \mathbf{I}_{L_{\mathrm{b}}} \mathbf{A}_{\mathrm{b}} \diamond \mathbf{I}_{L_{\mathrm{c}}} \mathbf{A}_{\mathrm{c}} \diamond \cdots \diamond \mathbf{I}_{L_{\mathrm{u}}} \mathbf{A}_{\mathrm{u}}
\end{aligned}
$$

Using the mixed-product property of Kronecker product ${ }^{7}$, (4.50) can be shown to be

$$
\begin{aligned}
& \mathbf{A}_{\mathrm{a} 1}=\left(\mathbf{J}_{\mathrm{a} 1} \otimes \mathbf{I}_{L_{\mathrm{b}}} \otimes \mathbf{I}_{L_{\mathrm{c}}} \otimes \cdots \otimes \mathbf{I}_{L_{\mathrm{u}}}\right) \mathbf{A} \\
& \mathbf{A}_{\mathrm{a} 2}=\left(\mathbf{J}_{\mathrm{a} 2} \otimes \mathbf{I}_{L_{\mathrm{b}}} \otimes \mathbf{I}_{L_{\mathrm{c}}} \otimes \cdots \otimes \mathbf{I}_{L_{\mathrm{u}}}\right) \mathbf{A}
\end{aligned}
$$

${ }^{7}$ The mixed-product property of Kronecker product is defined as

$$
(\mathbf{A} \otimes \mathbf{B})(\mathbf{C} \otimes \mathbf{D})=\mathbf{A C} \otimes \mathbf{B D}
$$

Note that this property also applies to the Khatri-Rao product, so

$$
(\mathbf{A} \diamond \mathbf{B})(\mathbf{C} \diamond \mathbf{D})=\mathbf{A C} \otimes \mathbf{B D}
$$


Using (4.51), the invariance equation in (4.46) can be expressed in terms of the steering matrix $\mathbf{A}$,

$$
\mathbf{J}_{1 \mathrm{a}} \mathbf{A}=\mathbf{J}_{2 \mathrm{a}} \mathbf{A D}(\mathbf{a})
$$

where the selection matrices in (4.52) are given by

$$
\begin{aligned}
& \mathbf{J}_{1 \mathrm{a}}=\mathbf{J}_{\mathrm{a} 1} \otimes \mathbf{I}_{L_{\mathrm{b}}} \otimes \mathbf{I}_{L_{\mathrm{c}}} \otimes \cdots \otimes \mathbf{I}_{L_{\mathrm{u}}} \\
& \mathbf{J}_{2 \mathrm{a}}=\mathbf{J}_{\mathrm{a} 2} \otimes \mathbf{I}_{L_{\mathrm{b}}} \otimes \mathbf{I}_{L_{\mathrm{c}}} \otimes \cdots \otimes \mathbf{I}_{L_{\mathrm{u}}}
\end{aligned}
$$

Following the same procedure, the invariance equation in (4.52) can be obtained for the other dimensions in the multidimensional Vandermonde matrix.

\subsection{Prediction Schemes}

In this section we describe the proposed prediction schemes using the models developed in Section 4.1 and the transformed data described in Section 4.2. We will henceforth refer to the proposed schemes as Multidimensional ESPRIT based MIMO CHAnnel Predictor (MEMCHAP) and use the acronyms for the models to distinguish the algorithms. For example, the scheme based on MSSM will be referred to as MSSM-MEMCHAP and so on. The general concept of the proposed schemes is illustrated in Figure 4.1. Each of the method is divided into the following stages:

- Data transformation: As described in Section 4.2, this stage involves the transformation of the available channel matrices in a manner that a sufficiently large data matrix exhibiting the required translational invariance structure in all dimensions is obtained.

- Covariance matrix estimation: Based on the transformed data, the covariance matrix containing the temporal, receive spatial and/or transmit spatial correlations is estimated in this stage. 
- Source number estimation and subspace decomposition: Here, we estimate the number of sources and separate the subspace spanned by the data into signal and noise subspace.

- Joint parameter estimation: The invariance structure in the transformed data is exploited to jointly estimate the structural parameters of the channel.

- Amplitude estimation: Using the estimates of the structural parameters and the channel estimates, the amplitude parameters are estimated.

- Channel prediction: The last stage in the proposed schemes involve extrapolation of the CSI based on the parameter estimates and the models.

It should be noted that although the proposed methods have similar procedures, they differ in the model, dimension of parameter estimation and number of amplitude and structural parameters. As mentioned earlier, the various models allow for application across different systems (i.e., SIMO, MISO, MIMO). The difference in the number of parameters and estimation dimension is expected to result in varying estimation and prediction performance across the different methods. For instance, an increase in the number of dimension improves the accuracy of the parameter estimates even if there is a corresponding increase in the number of parameters

An illustration: Consider the problem of estimating the $P$ parameters $\left\{\eta_{p}\right\}_{p=1}^{P}$ and the $2 P$ parameters $\left\{\eta_{p}, \psi_{p}\right\}_{p=1}^{P}$ for an $N$-element $\mathrm{ULA}^{8}$ and an $N \times N$ UPA, respectively. In the ULA case, the observation per parameter is $N / P$ while the UPA approach has $N^{2} / 2 P$ observation per parameter. Since $N^{2} / 2 P>N / P$, the two-dimensional approach has more observations and should perform better than the one-dimensional ULA estimation.

\footnotetext{
${ }^{8} N$ refers to the number of accessible antenna elements. Each antenna element may consist of a number of component antennas whose measurements can not be accessed.
} 


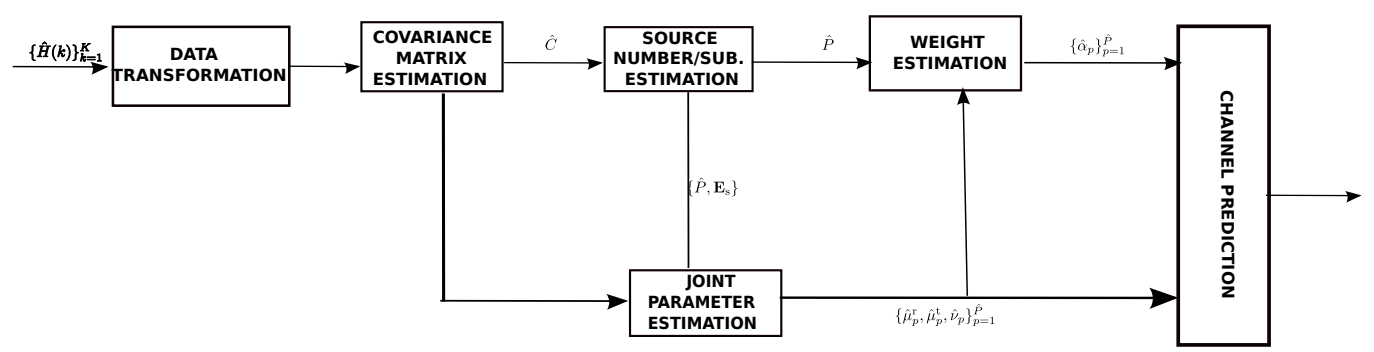

Figure 4.1: A block illustration of the stages in the proposed MEMCHAP schemes.

\subsubsection{MSSM - MEMCHAP}

The MSSM-MEMCHAP requires the estimation of $P$ Doppler frequencies which can be achieved via a 1D ESPRIT based method. This method is therefore an application of the SISO predictions schemes in [24,195] to narrowband MIMO channels. Based on the assumption that the channel between each antenna pair experiences the same Doppler shift, the covariance estimate is averaged over all entries in the CSI matrix. The steps in the MSSM-MEMCHAP are presented below.

\section{Covariance Matrix Estimation}

The temporal correlation matrix is obtained from (4.38), giving

$$
\hat{\mathbf{C}}_{\mathrm{m}}=\frac{1}{N M T_{\mathrm{m}}} \hat{\mathcal{H}}_{\mathrm{m}} \hat{\mathcal{H}}_{\mathrm{m}}^{H}
$$

Using (4.39), (4.54) can be expressed as

$$
\begin{aligned}
\hat{\mathbf{C}}_{\mathrm{m}} & =\frac{1}{N M T_{\mathrm{m}}} \sum_{c=1}^{N M T_{\mathrm{m}}}(\mathbf{A}(\boldsymbol{\nu}) \boldsymbol{\beta}(c)+\mathbf{w}(c))(\mathbf{A}(\boldsymbol{\nu}) \boldsymbol{\beta}(c)+\mathbf{w}(c))^{H} \\
& =\frac{1}{N M T_{\mathrm{m}}} \sum_{c=1}^{N M T_{\mathrm{m}}}\left(\mathbf{A}(\boldsymbol{\nu}) \boldsymbol{\beta}(c) \boldsymbol{\beta}(c)^{H} \mathbf{A}(\boldsymbol{\nu})^{H}+2 \mathbf{A}(\boldsymbol{\nu}) \boldsymbol{\beta}(c) \mathbf{w}(c)+\mathbf{w}(c) \mathbf{w}(c)^{H}\right) \\
& =\underbrace{\mathbf{A}(\boldsymbol{\nu}) \hat{\mathbf{C}}_{\beta \beta} \mathbf{A}(\boldsymbol{\nu})^{H}}_{\text {signal }}+\underbrace{\sigma^{2} \mathbf{I}}_{\text {noise }}
\end{aligned}
$$


where we have assumed that the noise and signal are uncorrelated such that $\mathbb{E}(\mathbf{A}(\boldsymbol{\nu}) \boldsymbol{\beta}(c) \mathbf{w}(c))=0$ and that the noise is temporally and spatially white. Note that we use the same assumption throughout the thesis. The covariance $\hat{\mathbf{C}}_{\beta \beta}$ is defined as

$$
\hat{\mathbf{C}}_{\beta \beta}=\frac{1}{N M T_{\mathrm{m}}} \sum_{c=1}^{N M T_{\mathrm{m}}} \boldsymbol{\beta} \boldsymbol{\beta}^{H}
$$

As shown in (4.4), the temporal correlation matrix can be separated into signal and noise components. The signal component is a function of the Doppler frequencies and will be used as a basis for their estimation.

\section{Source Number Estimation and Subspace Decomposition}

We estimate the number of dominant paths using the Minimum Mean Square Error Minimum Description Length (MMDL) criterion [85] in (3.57), giving

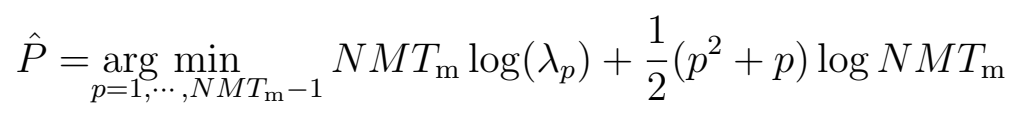

where $\lambda_{p}$ are the eigenvalues of $\hat{\mathbf{C}_{\mathrm{m}}}$. Using (3.8), the EVD of $\hat{\mathbf{C}_{\mathrm{m}}}$ can now be decomposed into signal and noise subspaces, denoted by $\hat{\mathbf{E}}_{\mathrm{s}}$ and $\hat{\mathbf{E}}_{\mathrm{n}}$, respectively. It should be noted that the eigenvalues in (4.57) are those of the covariance matrix obtained from the transformed data matrix and not the MIMO channel matrix itself.

\section{Doppler Frequency Estimation}

Estimation of the normalized Doppler frequencies $\{\nu\}_{p=1}^{\hat{P}}$ is based on the ESPRIT algorithm presented in Section 3.2. This is achieved by first solving (3.23) for $\hat{\boldsymbol{\Psi}}$. The normalized Doppler frequency for the $p$ th path is thus

$$
\hat{\nu}_{p}=\arg \left[\lambda_{p}\right]
$$

Here, $\lambda_{p}$ is the $p$ th eigenvalue of $\hat{\mathbf{\Psi}}$. 


\section{MSS Estimation}

Using the estimated Doppler frequencies, the $K$ noisy CSI estimates can be written in terms of the MSSM in (4.13), giving

$$
\hat{\mathbf{H}}(k)=\sum_{p=1}^{P} \mathbf{S}_{p} e^{j k \hat{\nu}_{p}}+\mathbf{W}(k) ; k=0,1, \cdots, K-1
$$

which can be expressed in an expanded form as

$$
\left[\begin{array}{ccc}
\hat{h}_{11}(k) & \cdots & \hat{h}_{1 N}(k) \\
\hat{h}_{21}(k) & \cdots & \hat{h}_{2 N}(k) \\
\vdots & \ddots & \vdots \\
\hat{h}_{M 1}(k) & \cdots & \hat{h}_{M N}(k)
\end{array}\right]=\sum_{p=1}^{P}\left\{\left[\begin{array}{ccc}
\mathbf{S}_{p}(1,1) & \cdots & \mathbf{S}_{p}(1, N) \\
\mathbf{S}_{p}(2,1) & \cdots & \mathbf{S}_{p}(2, N) \\
\vdots & \ddots & \vdots \\
\mathbf{S}_{p}(M, 1) & \cdots & \mathbf{S}_{p}(M, N)
\end{array}\right] e^{j k \hat{\nu}_{p}}\right\}+\mathbf{W}(k)
$$

Performing a vectorization operation on (4.60) yields

$$
\hat{\mathbf{h}}(k)=\sum_{p=1}^{P} \mathbf{s}_{p} e^{j k \hat{\nu}_{p}}+\mathbf{w}(k) ; k=0,1, \cdots, K-1
$$

where $\mathbf{s}_{p}$ is the $N M \times 1$ vectorized form of the MSS for the $p$ th path. More compactly, (4.61) can be written as

$$
\hat{\mathbf{h}}(k)=\mathbf{S} \hat{\mathbf{f}}(k)+\mathbf{w}(k) ; \quad k=0,1, \cdots, K-1
$$

where

$$
\begin{aligned}
\mathbf{S} & =\left[\begin{array}{llll}
\mathbf{s}_{1} & \mathbf{s}_{2} & \cdots & \mathbf{s}_{P}
\end{array}\right] \in \mathcal{C}^{N M \times P} \\
\hat{\mathbf{f}}(k) & =\left[\begin{array}{llll}
e^{j k \hat{\nu}_{1}} & e^{j k \hat{\nu}_{2}} & \cdots & e^{j k \hat{\nu}_{P}}
\end{array}\right]^{T} \in \mathcal{C}^{P \times 1}
\end{aligned}
$$

By combining the $K$ equations in (4.62), we obtain

$$
\hat{\mathbf{H}}=\mathbf{S} \hat{\mathbf{F}}+\mathbf{W}
$$


Here, $\hat{\mathbf{H}} \in \mathcal{C}^{N M \times K}$ is a matrix containing the vectorized versions of the $K$ known CSI values and $\hat{\mathbf{F}} \in \mathcal{C}^{P \times K}$ is the Vandermonde matrix

$$
\hat{\mathbf{F}}=\left[\begin{array}{ccccc}
1 & e^{j \hat{\nu}_{1}} & e^{j 2 \hat{\nu}_{1}} & \cdots & e^{j(K-1) \hat{\nu}_{1}} \\
1 & e^{j \hat{\nu}_{2}} & e^{j 2 \hat{\nu}_{2}} & \cdots & e^{j(K-1) \hat{\nu}_{2}} \\
\vdots & \vdots & \vdots & \ddots & \vdots \\
1 & e^{j \hat{\nu}_{\hat{P}}} & e^{j 2 \hat{\nu}_{\hat{P}}} & \cdots & e^{j(K-1) \hat{\nu}_{\hat{P}}}
\end{array}\right]
$$

Solving (4.62) in the least square sense yields an estimate of $\mathbf{S}$ given by

$$
\hat{\mathbf{S}}=\left(\hat{\mathbf{F}}^{H} \hat{\mathbf{F}}+\eta \mathbf{I}\right) \hat{\mathbf{F}}^{H} \hat{\mathbf{H}}
$$

where $\eta$ is a regularization parameter chosen to minimize the effects of errors in $\hat{\mathbf{F}}$ on the weight estimation. Throughout this chapter, $\eta$ is chosen empirically as $10^{-5}$. This value is chosen after trials with values of $\eta$ between 0 and $10^{-10}$. Our investigations show that values in this range result in similar prediction performance. However, the unregularized equivalent (i.e $\eta=0$ ) should be avoided with low SNR values.

An alternative approach for the MSS estimation is to form $K$ equations for each entries of the CSI matrix and solve for corresponding entries of the MSS for each path. Let $\mathbf{s}_{m n}=\left[\begin{array}{llll}\mathbf{S}_{1}(m, n) & \mathbf{S}_{2}(m, n) & \cdots & \mathbf{S}_{P}(m, n)\end{array}\right] \in \mathcal{C}^{P \times 1}$ be a vector containing the $(m, n)$ entry of the MSS for all paths. Using (4.59), the $K$ equations for the $(m, n)$ of the CSI matrix is obtained as

$$
\hat{\mathbf{h}}_{m n}=\hat{\mathbf{G}} \mathbf{s}_{m n}+\mathbf{w}
$$

for $m \in[1, M]$ and $n \in[1, N]$.

In (4.67), $\hat{\mathbf{h}}_{m n}=\left[\begin{array}{llll}\hat{h}_{m n}(0) & \hat{h}_{m n}(1) & \cdots & \hat{h}_{m n}(K-1)\end{array}\right]$ and $\hat{\mathbf{G}}=\hat{\mathbf{F}}^{T}$. Using the least square estimates of $\mathbf{s}_{m n}$ from (4.67), the MSS for the $p$ th path is computed as

$$
\hat{\mathbf{S}}_{p}=\left[\begin{array}{cccc}
\hat{\mathbf{s}}_{11}(p) & \hat{\mathbf{s}}_{12}(p) & \cdots & \hat{\mathbf{s}}_{1 N}(p) \\
\hat{\mathbf{s}}_{21}(p) & \hat{\mathbf{s}}_{22}(p) & \cdots & \hat{\mathbf{s}}_{2 N}(p) \\
\vdots & \vdots & \ddots & \vdots \\
\hat{\mathbf{s}}_{M 1}(p) & \hat{\mathbf{s}}_{M 2}(p) & \cdots & \hat{\mathbf{s}}_{M N}(p)
\end{array}\right]
$$




\section{Channel Prediction}

Once the Doppler frequencies and MSS have been estimated, extrapolation of the CSI via the MSSM approach is achieved using

$$
\tilde{\mathbf{H}}(k)=\sum_{p=1}^{\hat{P}} \hat{\mathbf{S}}_{p} e^{j(k-1) \hat{\nu}_{p}}
$$

\subsubsection{RSSM - MEMCHAP}

Unlike the MSSM-MEMCHAP approach, the RSSM-MEMCHAP involves 2D parameter estimation. The steps involved in the RSSM-MEMCHAP are as follows.

\section{Covariance Matrix Estimation}

The covariance matrix containing the transmit spatial and temporal correlations of the MIMO channel is estimated from (4.34) as

$$
\hat{\mathbf{C}}_{\mathrm{r}}=\frac{1}{M T_{\mathrm{r}}} \hat{\mathcal{H}}_{\mathrm{r}} \hat{\mathcal{H}}_{\mathrm{r}}^{H}
$$

Using (4.36), (4.70) can be expressed as

$$
\begin{aligned}
\hat{\mathbf{C}}_{\mathrm{r}}= & \frac{1}{M T_{\mathrm{r}}} \sum_{c=1}^{M T_{\mathrm{r}}}\left(\mathbf{A}\left(\boldsymbol{\mu}^{\mathrm{t}}, \boldsymbol{\nu}\right) \boldsymbol{\beta}(c)+\mathbf{w}(c)\right)\left(\mathbf{A}\left(\boldsymbol{\mu}^{\mathrm{t}}, \boldsymbol{\nu}\right) \boldsymbol{\beta}(c)+\mathbf{w}(c)\right)^{H} \\
= & \frac{1}{M T_{\mathrm{m}}} \sum_{c=1}^{M T_{\mathrm{r}}}\left(\mathbf{A}\left(\boldsymbol{\mu}^{\mathrm{t}}, \boldsymbol{\nu}\right) \boldsymbol{\beta}(c) \boldsymbol{\beta}(c)^{H} \mathbf{A}\left(\boldsymbol{\mu}^{\mathrm{t}}, \boldsymbol{\nu}\right)^{H}+2 \mathbf{A}\left(\boldsymbol{\mu}^{\mathrm{t}}, \boldsymbol{\nu}\right) \boldsymbol{\beta}(c) \mathbf{w}(c)\right. \\
& \left.\quad+\mathbf{w}(c) \mathbf{w}(c)^{H}\right) \\
= & \underbrace{\mathbf{A}\left(\boldsymbol{\mu}^{\mathrm{t}}, \boldsymbol{\nu}\right) \hat{\mathbf{C}}_{\beta \beta} \mathbf{A}\left(\boldsymbol{\mu}^{\mathrm{t}}, \boldsymbol{\nu}\right)^{H}}_{\text {signal }}+\underbrace{\sigma^{2} \mathbf{I}}_{\text {noise }}
\end{aligned}
$$

Here

$$
\hat{\mathbf{C}}_{\beta \beta}=\frac{1}{M T_{\mathrm{r}}} \sum_{c=1}^{M T_{\mathrm{r}}} \boldsymbol{\beta} \boldsymbol{\beta}^{H}
$$


The signal component of (4.71) is a function of the transmit spatial frequencies $\left\{\mu_{p}^{\mathrm{t}}\right\}_{p=1}^{P}$ and normalized Doppler frequencies $\left\{\nu_{p}\right\}_{p=1}^{P}$. These parameters can therefore be extracted jointly from the transformed RSSM data via a $2 \mathrm{D}$ extension of the ESPRIT method.

\section{Source Number Estimation and Subspace Decomposition}

Using the MMDL criterion, the number of paths is estimated in the TSSM scheme as

$$
\hat{P}=\underset{p=1, \cdots, N R_{\mathrm{r}}-1}{\operatorname{argmin}} N R_{\mathrm{r}} \log \left(\lambda_{p}\right)+\frac{1}{2}\left(p^{2}+p\right) \log N R_{\mathrm{r}}
$$

where $\lambda_{p}$ are the eigenvalues of $\hat{\mathbf{C}}_{\mathrm{r}}$. Again, the signal subspace eigenvector matrix $\mathbf{E}_{\mathrm{s}}$ is obtained by decomposing $\hat{\mathbf{C}}_{\mathrm{r}}$ using (3.8).

\section{Joint Parameter Estimation}

Estimation of the parameters $\left\{\mu_{p}^{\mathrm{t}}, \nu_{p}\right\}_{p=1}^{P}$ in the RSSM-MEMCHAP scheme requires four selection matrices obtained from (4.53) as

$$
\begin{aligned}
& \mathbf{J}_{\mathrm{d} 1}=\mathbf{I}_{N} \otimes \mathbf{J}_{1 \mathrm{~d}} \\
& \mathbf{J}_{\mathrm{d} 2}=\mathbf{I}_{N} \otimes \mathbf{J}_{2 \mathrm{~d}} \\
& \mathbf{J}_{\mathrm{r} 1}=\mathbf{J}_{1 \mathrm{r}} \otimes \mathbf{I}_{R_{\mathrm{r}}} \\
& \mathbf{J}_{\mathrm{r} 2}=\mathbf{J}_{2 \mathrm{r}} \otimes \mathbf{I}_{R_{\mathrm{r}}}
\end{aligned}
$$

With $N=3$ and $R_{\mathrm{r}}=3$, the selection matrices for the receive spatial dimension has the form

$$
\mathbf{J}_{\mathrm{r} 1}=\left[\begin{array}{lllllllll}
1 & 0 & 0 & 0 & 0 & 0 & 0 & 0 & 0 \\
0 & 1 & 0 & 0 & 0 & 0 & 0 & 0 & 0 \\
0 & 0 & 1 & 0 & 0 & 0 & 0 & 0 & 0 \\
0 & 0 & 0 & 1 & 0 & 0 & 0 & 0 & 0 \\
0 & 0 & 0 & 0 & 1 & 0 & 0 & 0 & 0 \\
0 & 0 & 0 & 0 & 0 & 1 & 0 & 0 & 0
\end{array}\right]
$$


and

$$
\mathbf{J}_{\mathrm{r} 2}=\left[\begin{array}{lllllllll}
0 & 0 & 0 & 1 & 0 & 0 & 0 & 0 & 0 \\
0 & 0 & 0 & 0 & 1 & 0 & 0 & 0 & 0 \\
0 & 0 & 0 & 0 & 0 & 1 & 0 & 0 & 0 \\
0 & 0 & 0 & 0 & 0 & 0 & 1 & 0 & 0 \\
0 & 0 & 0 & 0 & 0 & 0 & 0 & 1 & 0 \\
0 & 0 & 0 & 0 & 0 & 0 & 0 & 0 & 1
\end{array}\right]
$$

In the Doppler/temporal dimension, the selection matrices are

$$
\mathbf{J}_{\mathrm{d} 1}=\left[\begin{array}{ccccccccc}
1 & 0 & 0 & 0 & 0 & 0 & 0 & 0 & 0 \\
0 & 1 & 0 & 0 & 0 & 0 & 0 & 0 & 0 \\
0 & 0 & 0 & 1 & 0 & 0 & 0 & 0 & 0 \\
0 & 0 & 0 & 0 & 1 & 0 & 0 & 0 & 0 \\
0 & 0 & 0 & 0 & 0 & 0 & 1 & 0 & 0 \\
0 & 0 & 0 & 0 & 0 & 0 & 0 & 1 & 0
\end{array}\right]
$$

and

$$
\mathbf{J}_{\mathrm{d} 2}=\left[\begin{array}{lllllllll}
0 & 1 & 0 & 0 & 0 & 0 & 0 & 0 & 0 \\
0 & 0 & 1 & 0 & 0 & 0 & 0 & 0 & 0 \\
0 & 0 & 0 & 0 & 1 & 0 & 0 & 0 & 0 \\
0 & 0 & 0 & 0 & 0 & 1 & 0 & 0 & 0 \\
0 & 0 & 0 & 0 & 0 & 0 & 0 & 1 & 0 \\
0 & 0 & 0 & 0 & 0 & 0 & 0 & 0 & 1
\end{array}\right]
$$

Using (4.74), we form the invariance equations

$$
\begin{aligned}
\mathbf{J}_{\mathrm{d} 1} \hat{\mathbf{E}}_{\mathrm{s}} & =\mathbf{J}_{\mathrm{d} 2} \hat{\mathbf{E}}_{\mathrm{s}} \boldsymbol{\Phi}_{\mathrm{d}} \\
\mathbf{J}_{\mathrm{r} 1} \hat{\mathbf{E}}_{\mathrm{s}} & =\mathbf{J}_{\mathrm{r} 2} \hat{\mathbf{E}}_{\mathrm{s}} \boldsymbol{\Phi}_{\mathrm{r}}
\end{aligned}
$$

which can be solved via a LS approach to obtain

$$
\begin{aligned}
& \boldsymbol{\Phi}_{\mathrm{d}}=\left(\mathbf{J}_{\mathrm{d} 2} \hat{\mathbf{E}}_{\mathrm{s}}\right)^{\dagger} \mathbf{J}_{\mathrm{d} 1} \hat{\mathbf{E}}_{\mathrm{s}} \\
& \boldsymbol{\Phi}_{\mathrm{r}}=\left(\mathbf{J}_{\mathrm{r} 2} \hat{\mathbf{E}}_{\mathrm{s}}\right)^{\dagger} \mathbf{J}_{\mathrm{r} 1} \hat{\mathbf{E}}_{\mathrm{s}}
\end{aligned}
$$


The transition matrices in (4.80) satisfy

$$
\begin{aligned}
& \operatorname{eig}\left[\boldsymbol{\Phi}_{\mathrm{d}}\right]=\mathbf{D}(\boldsymbol{\nu}) \\
& \operatorname{eig}\left[\boldsymbol{\Phi}_{\mathrm{r}}\right]=\mathbf{D}\left(\boldsymbol{\mu}_{\mathbf{r}}\right)
\end{aligned}
$$

where eig $[\cdot]$ returns the diagonal eigenvalue matrix of the associated matrix. Equation (4.81) is based on the observation that $\mathbf{E}_{s}$ and $\mathbf{A}$ are rotated versions of each other in the same signal subspace [126]. It should be noted that although estimates of the AOAs and normalized Doppler shifts can be obtained directly from (4.81), an additional algorithm to pair the estimates is required. Typically, pairing of multiple parameter estimates is achieved by using joint Schur decomposition [7,74] or simultaneous diagonalization $[38,63]$. These schemes, however, significantly increase the complexity of the algorithm. Instead, automatic pairing of the estimates is achieved in this thesis using a scheme similar to the mean eigenvalue decomposition (MEVD) [141]. Let the eigendecomposition of the sum of (4.81) be

$$
\begin{aligned}
\boldsymbol{\Phi} & =\boldsymbol{\Phi}_{\mathrm{r}}+\boldsymbol{\Phi}_{\mathrm{d}} \\
& =\mathbf{T}^{-1} \boldsymbol{\Lambda} \mathbf{T}
\end{aligned}
$$

Using (4.81), we obtain

$$
\begin{aligned}
\mathbf{D}(\boldsymbol{\nu}) & =\mathbf{T} \boldsymbol{\Phi}_{\mathrm{d}} \mathbf{T}^{-1} \\
\mathbf{D}\left(\boldsymbol{\mu}_{\mathbf{r}}\right) & =\mathbf{T} \boldsymbol{\Phi}_{\mathrm{r}} \mathbf{T}^{-1}
\end{aligned}
$$

Estimates of the direction cosines can now be obtained from (4.83) thus

$$
\begin{aligned}
\hat{\boldsymbol{\mu}}_{\mathrm{r}} & =\arg \left[\operatorname{diag}\left\{\mathbf{D}\left(\boldsymbol{\mu}_{\mathrm{r}}\right)\right\}\right] \\
\hat{\boldsymbol{\nu}} & =\arg [\operatorname{diag}\{\mathbf{D}(\boldsymbol{\nu})\}]
\end{aligned}
$$

\section{RSS Estimation}

Similar to the MSS estimation, the RSS can be estimated via a LS approach. Let $\mathbf{s}^{m}=\left[\begin{array}{llll}\mathbf{s}_{1}(m) & \mathbf{s}_{2}(m) & \cdots & \mathbf{s}_{\hat{P}}(m)\end{array}\right]^{T}$ be a vector containing the $m$ th 
entry of the RSS for all paths. Using (4.11), we obtain

$$
\hat{\mathbf{h}}_{m}=\mathbf{G}_{m} \mathbf{s}^{m}+\mathbf{z} \quad \forall m \in[1, M]
$$

where $\hat{\mathbf{h}}_{m}=\left[\hat{h}_{m 1}(1), \hat{h}_{m 1}(2), \cdots, \hat{h}_{1 m}(K)\right]^{T}$ and $\mathbf{G}_{m}$ is defined as

$$
\mathbf{G}_{m}=\mathbf{A}_{\mathrm{t}} \diamond \mathbf{G}
$$

where $\mathbf{G}$ is the Vandermonde matrix in (4.65) and $\mathbf{A}_{\mathrm{t}}$ is the transmit array steering matrix (4.26).

\section{Channel prediction}

Prediction of the channel using the RSSM-MEMCHAP is achieved using

$$
\tilde{\mathbf{H}}(k)=\sum_{p=1}^{\hat{P}} \hat{\mathbf{s}}_{p}^{\mathrm{r}} \mathbf{a}_{\mathrm{t}}^{T}\left(\hat{\mu}_{p}^{\mathrm{t}}\right) e^{j k \hat{\nu}_{p}}
$$

\subsubsection{TSSM - MEMCHAP}

Similar to the RSSM-MEMCHAP approach, the TSSM-MEMCHAP algorithm involves estimation of the receive spatial frequencies $\left\{\mu_{p}^{\mathrm{r}}\right\}_{p=1}^{P}$ and the normalized Doppler frequencies $\left\{\nu_{p}\right\}_{p=1}^{P}$. As before, we present a summary of the steps in the TSSM-MEMCHAP in the sequel.

\section{Covariance matrix Estimation}

Here we estimate the spatio-temporal covariance matrix $\hat{\mathbf{C}}_{\mathrm{t}}$ from (4.32) thus

$$
\hat{\mathbf{C}}_{\mathrm{t}}=\frac{1}{N T_{\mathrm{t}}} \hat{\mathcal{H}}_{\mathrm{t}} \hat{\mathcal{H}}_{\mathrm{t}}^{H}
$$


Using (4.33), (4.88) can be shown to be

$$
\begin{aligned}
\hat{\mathbf{C}}_{\mathrm{t}}= & \frac{1}{N T_{\mathrm{t}}} \sum_{c=1}^{N T_{\mathrm{t}}}\left(\mathbf{A}\left(\boldsymbol{\mu}^{\mathrm{r}}, \boldsymbol{\nu}\right) \boldsymbol{\beta}(c)+\mathbf{w}(c)\right)\left(\mathbf{A}\left(\boldsymbol{\mu}^{\mathrm{r}}, \boldsymbol{\nu}\right) \boldsymbol{\beta}(c)+\mathbf{w}(c)\right)^{H} \\
= & \frac{1}{N T_{\mathrm{t}}} \sum_{c=1}^{N T_{\mathrm{t}}}\left(\mathbf{A}\left(\boldsymbol{\mu}^{\mathrm{r}}, \boldsymbol{\nu}\right) \boldsymbol{\beta}(c) \boldsymbol{\beta}(c)^{H} \mathbf{A}\left(\boldsymbol{\mu}^{\mathrm{r}}, \boldsymbol{\nu}\right)^{H}+2 \mathbf{A}\left(\boldsymbol{\mu}^{\mathrm{r}}, \boldsymbol{\nu}\right) \boldsymbol{\beta}(c) \mathbf{w}(c)\right) \\
& \quad+\mathbf{w}(c) \mathbf{w}(c)^{H} \\
= & \underbrace{\mathbf{A}\left(\boldsymbol{\mu}^{\mathrm{r}}, \boldsymbol{\nu}\right) \hat{\mathbf{C}}_{\beta \beta} \mathbf{A}\left(\boldsymbol{\mu}^{\mathrm{r}}, \boldsymbol{\nu}\right)^{H}}_{\text {signal }}+\underbrace{\sigma^{2} \mathbf{I}}_{\text {noise }}
\end{aligned}
$$

where $\hat{\mathbf{C}}_{\beta \beta}$ is defined in (4.72).

\section{Source Number Estimation and Subspace Decomposition}

We estimate the number of paths using the MMDL criterion thus

$$
\hat{P}=\underset{p=1, \cdots, M R_{\mathrm{t}}-1}{\operatorname{argmin}} M R_{\mathrm{t}} \log \left(\lambda_{p}\right)+\frac{1}{2}\left(p^{2}+p\right) \log M R_{\mathrm{t}}
$$

where $\lambda_{p}$ are the eigenvalues of $\hat{\mathbf{C}}_{\mathrm{t}}$. Again, the signal subspace eigenvectors $\mathbf{E}_{\mathrm{s}}$ is obtained by decomposing $\hat{\mathbf{C}}_{\mathrm{t}}$ using (3.8).

\section{Joint Parameter Estimation}

As in the RSSM-MEMCHAP, the parameter estimation stage of the TSSMMEMCHAP is based on 2D extension of the ESPRIT method. We begin by defining selection matrices $\mathbf{J}_{\mathrm{d} 1}, \mathbf{J}_{\mathrm{d} 2}, \mathbf{J}_{\mathrm{r} 1}$ and $\mathbf{J}_{\mathrm{r} 2}$ by replacing $N$ and $R_{\mathrm{r}}$ in (4.74) by $M$ and $R_{\mathrm{t}}$, respectively. Using (4.79)-(4.83) with $\boldsymbol{\Phi}_{\mathrm{r}}$ replaced by $\boldsymbol{\Phi}_{\mathrm{t}}$, the structural parameters of the TSSM are obtained as

$$
\begin{aligned}
\hat{\boldsymbol{\mu}}_{\mathrm{r}} & =\arg \left[\operatorname{diag}\left\{\mathbf{D}\left(\boldsymbol{\mu}_{\mathrm{r}}\right)\right\}\right] \\
\hat{\boldsymbol{\nu}} & =\arg [\operatorname{diag}\{\mathbf{D}(\boldsymbol{\nu})\}]
\end{aligned}
$$




\section{TSS Estimation}

Let $\mathbf{s}^{n}=\left[\begin{array}{llll}\mathbf{s}_{1}(n) & \mathbf{s}_{2}(n) & \cdots & \mathbf{s}_{\hat{P}}(n)\end{array}\right]^{T}$ be a vector containing the $n$th entry of the TSS for all paths. Using (4.12), we obtain

$$
\hat{\mathbf{h}}_{n}=\mathbf{G}_{n} \mathbf{s}^{n}+\mathbf{z} \quad \forall n \in[1, N]
$$

where $\hat{\mathbf{h}}_{n}=\left[\hat{h}_{1 n}(1), \hat{h}_{1 n}(2), \cdots, \hat{h}_{1 n}(K), \hat{h}_{2 n}(1), \cdots, \hat{h}_{M n}(K)\right]^{T}$ and $\mathbf{G}_{n}$ is defined as

$$
\mathbf{G}_{n}=\mathbf{A}_{\mathbf{r}} \diamond \mathbf{G}
$$

Here, $\mathbf{A}_{\mathrm{r}}$ is the receive array steering matrix (4.26). We again solve (4.92) via a LS approach for the TSS.

\section{Channel Prediction}

As before, the predicted CSI using the TSSM-MEMCHAP approach is defined as

$$
\tilde{\mathbf{H}}(k)=\sum_{p=1}^{\hat{P}} \hat{\mathbf{s}}_{p}^{\mathrm{t}} \mathbf{a}_{\mathrm{r}}\left(\hat{\mu}_{p}^{\mathrm{r}}\right) e^{j k \hat{\nu}_{p}}
$$

\subsubsection{DOD/DOA - MEMCHAP}

The steps in the DOD/DOA-MEMCHAP approach are as follows.

\section{Covariance Matrix Estimation}

The spatio-temporal covariance matrix $\hat{\mathbf{C}}_{\mathrm{d}}$ containing the receive spatial, temporal and transmit spatial correlation is estimated from (4.17) thus

$$
\hat{\mathbf{C}}_{\mathrm{d}}=\frac{1}{T_{\mathrm{d}}} \hat{\mathcal{H}}_{\mathrm{d}} \hat{\mathcal{H}}_{\mathrm{d}}^{H}
$$


which can be shown using (4.22) to be

$$
\begin{aligned}
\hat{\mathbf{C}}_{\mathrm{d}}= & \frac{1}{T_{\mathrm{d}}} \sum_{c=1}^{T_{\mathrm{d}}}\left(\mathbf{A}\left(\boldsymbol{\mu}^{\mathrm{r}}, \boldsymbol{\mu}^{\mathrm{t}}, \boldsymbol{\nu}\right) \boldsymbol{\beta}(c)+\mathbf{w}(c)\right)\left(\mathbf{A}\left(\boldsymbol{\mu}^{\mathrm{t}}, \boldsymbol{\mu}^{\mathrm{t}}, \boldsymbol{\nu}\right) \boldsymbol{\beta}(c)+\mathbf{w}(c)\right)^{H} \\
= & \frac{1}{T_{\mathrm{d}}} \sum_{c=1}^{T_{\mathrm{d}}}\left(\mathbf{A}\left(\boldsymbol{\mu}^{\mathrm{r}}, \boldsymbol{\mu}^{\mathrm{t}}, \boldsymbol{\nu}\right) \boldsymbol{\beta}(c) \boldsymbol{\beta}(c)^{H} \mathbf{A}\left(\boldsymbol{\mu}^{\mathrm{r}}, \boldsymbol{\mu}^{\mathrm{t}}, \boldsymbol{\nu}\right)^{H}+2 \mathbf{A}\left(\boldsymbol{\mu}^{\mathrm{t}}, \boldsymbol{\nu}\right) \boldsymbol{\beta}(c) \mathbf{w}(c)\right) \\
& \quad+\mathbf{w}(c) \mathbf{w}(c)^{H} \\
= & \underbrace{\mathbf{A}\left(\boldsymbol{\mu}^{\mathrm{r}}, \boldsymbol{\mu}^{\mathrm{t}}, \boldsymbol{\nu}\right) \hat{\mathbf{C}}_{\beta \beta} \mathbf{A}\left(\boldsymbol{\mu}^{\mathrm{r}}, \boldsymbol{\mu}^{\mathrm{t}}, \boldsymbol{\nu}\right)^{H}}_{\text {signal }}+\underbrace{\sigma^{2} \mathbf{I}}_{\text {noise }}
\end{aligned}
$$

As seen in (4.96), the signal component of $\hat{\mathbf{C}}_{\mathrm{d}}$ is a function of the $3 P$ parameters $\left\{\mu_{p}^{\mathrm{r}}, \mu_{p}^{\mathrm{t}}, \nu_{p}\right\}_{p=1}^{P}$. $\hat{\mathbf{C}}_{\mathrm{d}}$ will be used in a 3D ESPRIT approach to extract these parameters.

\section{Source Number Estimation and Subspace Decomposition}

We estimate the number of paths using the MMDL criterion thus

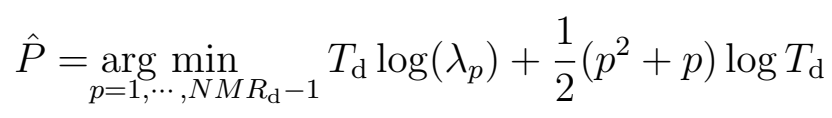

Here, $\lambda_{p}$ are the eigenvalues of $\hat{\mathbf{C}}_{\mathrm{d}}$ and the signal subspace eigenvector matrix $\mathbf{E}_{\mathrm{s}}$ is obtained by decomposing $\hat{\mathbf{C}}_{\mathrm{d}}$ using (3.8).

\section{Joint Parameter Estimation}

Using (4.53), we form six selection matrices (two for each dimension in the transformed data) thus

$$
\begin{aligned}
& \mathbf{J}_{\mathrm{d} 1}=\mathbf{I}_{M} \otimes \mathbf{I}_{N} \otimes \mathbf{J}_{1 \mathrm{~d}} \\
& \mathbf{J}_{\mathrm{d} 2}=\mathbf{I}_{M} \otimes \mathbf{I}_{N} \otimes \mathbf{J}_{2 \mathrm{~d}} \\
& \mathbf{J}_{\mathrm{t} 1}=\mathbf{I}_{M} \otimes \mathbf{J}_{1 \mathrm{t}} \otimes \mathbf{I}_{R_{\mathrm{d}}} \\
& \mathbf{J}_{\mathrm{t} 2}=\mathbf{I}_{M} \otimes \mathbf{J}_{2 \mathrm{t}} \otimes \mathbf{I}_{R_{\mathrm{d}}} \\
& \mathbf{J}_{\mathrm{r} 1}=\mathbf{J}_{1 \mathrm{r}} \otimes \mathbf{I}_{N} \otimes \mathbf{I}_{R_{\mathrm{d}}} \\
& \mathbf{J}_{\mathrm{r} 2}=\mathbf{J}_{2 \mathrm{r}} \otimes \mathbf{I}_{N} \otimes \mathbf{I}_{R_{\mathrm{d}}}
\end{aligned}
$$


Using (4.98), we form the invariance equations

$$
\begin{aligned}
\mathbf{J}_{\mathrm{d} 1} \hat{\mathbf{E}}_{\mathrm{s}} & =\mathbf{J}_{\mathrm{d} 2} \hat{\mathbf{E}}_{\mathrm{s}} \boldsymbol{\Phi}_{\mathrm{d}} \\
\mathbf{J}_{\mathrm{r} 1} \hat{\mathbf{E}}_{\mathrm{s}} & =\mathbf{J}_{\mathrm{r} 2} \hat{\mathbf{E}}_{\mathrm{s}} \boldsymbol{\Phi}_{\mathrm{r}} \\
\mathbf{J}_{\mathrm{t} 1} \hat{\mathbf{E}}_{\mathrm{s}} & =\mathbf{J}_{\mathrm{t} 2} \hat{\mathbf{E}}_{\mathrm{s}} \boldsymbol{\Phi}_{\mathrm{t}}
\end{aligned}
$$

which are solved to obtain the transition matrices $\hat{\boldsymbol{\Phi}}_{\mathrm{d}}, \hat{\boldsymbol{\Phi}}_{\mathrm{r}}$ and $\hat{\boldsymbol{\Phi}}_{\mathrm{t}}$. Again, we use the MEVD to achieve automatic pairing of the estimates. Estimates of the parameters are then obtained from the corresponding transition matrix using (4.84).

\section{Complex Amplitude Estimation}

We assume that the complex amplitude of each path is equal for all antenna pairs, which is reasonable considering the separation of gain from array dependent steering vectors in (4.2). The complex amplitudes can therefore be estimated via a least square fit to the known channel. Using the Vandermonde structure of the steering matrix and (4.2), we form the following equation for the first entry of $\hat{\mathbf{H}}$

$$
\left[\begin{array}{c}
\hat{h}_{11}(1) \\
\hat{h}_{11}(2) \\
\vdots \\
\hat{h}_{11}(K)
\end{array}\right]=\left[\begin{array}{ccc}
1 & \cdots & 1 \\
e^{j \hat{\nu}_{1}} & \cdots & e^{j \hat{\nu}_{\hat{P}}} \\
\vdots & \ddots & \vdots \\
e^{j(K-1) \hat{\nu}_{1}} & \cdots & e^{j(K-1) \hat{\nu}_{\hat{P}}}
\end{array}\right]\left[\begin{array}{c}
\alpha_{1} \\
\alpha_{2} \\
\vdots \\
\alpha_{\hat{P}}
\end{array}\right]+\left[\begin{array}{c}
w(1) \\
w(2) \\
\vdots \\
w(K)
\end{array}\right]
$$

which can be written in matrix form as

$$
\hat{\mathbf{h}}_{11}=\hat{\mathbf{G}} \boldsymbol{\alpha}+\mathbf{w}
$$

In (4.101), $\boldsymbol{\alpha}$ can be obtained via a regularized least square solution

$$
\hat{\boldsymbol{\alpha}}=\left(\hat{\mathbf{G}}^{H} \hat{\mathbf{G}}+\sigma \mathbf{I}\right) \hat{\mathbf{G}}^{H} \hat{\mathbf{h}}_{11}
$$

where $\sigma$ is the regularization parameter chosen to minimize the effects of errors in $\hat{\mathbf{G}}$ on the estimation. Note that although (4.102) gives an estimate 
of the complex amplitudes, our preliminary simulations show that improved estimates can be obtained by using other entries of $\hat{\mathbf{H}}$ in the estimation. We therefore generalize (4.101) as

$$
\hat{\mathbf{h}}_{m n}=\hat{\mathbf{G}}_{m n} \boldsymbol{\alpha}+\mathbf{w} \quad \forall n \in[1, N] \quad m \in[1, M]
$$

with $\hat{\mathbf{G}}_{m n}$ defined as

$$
\hat{\mathbf{G}}_{m n}=\mathbf{v}_{m}^{\mathrm{r}} \diamond \hat{\mathbf{G}} \diamond \mathbf{v}_{n}^{\mathrm{t}}
$$

where $\mathbf{v}_{m}^{\mathrm{r}}$ is defined as

$$
\mathbf{v}_{m}^{\mathrm{r}}=\left[\begin{array}{llll}
e^{j(m-1) \mu_{1}^{\mathrm{r}}} & e^{j(m-1) \mu_{2}^{\mathrm{r}}} & \cdots & e^{j(m-1) \mu_{\hat{P}}^{\mathrm{r}}}
\end{array}\right]
$$

$\mathbf{v}_{n}^{\mathrm{t}}$ is defined analogously. We combine the $N M$ equations in (4.104) and solve for $\hat{\boldsymbol{\alpha}}$ as

$$
\hat{\boldsymbol{\alpha}}=\left(\hat{\mathbf{G}}_{\mathrm{d}}^{H} \hat{\mathbf{G}}_{\mathrm{d}}+\sigma \mathbf{I}\right) \hat{\mathbf{G}}_{\mathrm{d}}^{H} \hat{\mathbf{h}}
$$

where $\hat{\mathbf{h}}=\left[\begin{array}{llll}\hat{\mathbf{h}}_{11}^{T} & \hat{\mathbf{h}}_{12}^{T} & \cdots & \hat{\mathbf{h}}_{M N}^{T}\end{array}\right]^{T}$ and $\hat{\mathbf{G}}_{\mathrm{d}}=\left[\begin{array}{llll}\hat{\mathbf{G}}_{11} & \hat{\mathbf{G}}_{12} & \cdots & \hat{\mathbf{G}}_{M N}\end{array}\right]$. It should be noted that the choice of using (4.102) or (4.106) is a compromise between complexity and accuracy since the improved amplitude estimates in (4.106) is achieved at the cost of increased computational complexity. In this chapter, we will utilize (4.106) for our analysis.

\section{Channel Prediction}

Extrapolation of the CSI using the DOD/DOA-MEMCHAP is achieved via

$$
\tilde{\mathbf{H}}(k)=\sum_{p=1}^{\hat{P}} \hat{\alpha}_{p} \mathbf{a}_{\mathrm{r}}\left(\hat{\mu}_{p}^{\mathrm{r}}\right) \mathbf{a}_{\mathrm{t}}^{T}\left(\hat{\mu}_{p}^{\mathrm{t}}\right) e^{j k \hat{\nu}_{p}}
$$

Table 4.3 presents a comparison of the proposed methods in terms of model, array geometry dependence, and application.

\subsection{Application to 3D Propagation Scenarios}

In Sections 4.1-4.4, we have proposed different methods for the prediction of $2 \mathrm{D}$ narrowband MIMO channels. The methods are based on parametric 
Table 4.3: A comparison of the proposed methods

\begin{tabular}{llllll}
\hline \multirow{2}{*}{ Method } & MSSM & TSSM & RSSM & DOD/DOA \\
\hline \multirow{4}{*}{ Correlation } & Receive & No & Yes & No & Yes \\
& Transmit & No & No & Yes & Yes \\
& Temporal & Yes & Yes & Yes & Yes \\
\hline \multirow{4}{*}{ System } & SISO & Yes & No & No & No \\
& SIMO & Yes & Yes & No & No \\
& MISO & Yes & No & Yes & No \\
& MIMO & Yes & Yes & Yes & Yes \\
Array Geometry & Other & Yes & Yes & Yes & Yes \\
& Modification & No & Yes & Yes & Yes \\
\hline
\end{tabular}

modeling of the double directional channel. We utilized multidimensional ESPRIT for estimating the parameters of the channel (i.e., angles of arrival, angles of departure, Doppler frequency shifts and complex amplitudes) and extrapolated the channel using the estimated parameters. These schemes considered 2D propagation scenarios where the elevation spectrum is neglected. It has, however, been shown that neglecting the elevation spectrum impacts the accuracy of the model [171]. This is particularly true in scenarios where the mobile station is indoor or in-vehicle such that rays which are reflected from the floor and/or ceiling contribute significantly to the received signal power.

While there exists extensive literature on 3D MIMO channel modeling (see e.g $[6,171]$ and the references therein), no literature exists on the prediction of 3D channel models. Moreover, parametric estimation of channel parameters has been identified as a useful approach for channel estimation [197, 228] and localization [82] in massive MIMO systems. In this section, we 
extend our concept to parameter estimation and channel prediction in 3D MIMO systems. Although all the proposed methods can be extended to 3D prediction, we present only extension of the DOD/DOA-MEMCHAP ${ }^{9}$.

\subsubsection{Channel Model}

Consider the 3D propagation environment in Fig. 4.2. The 3D channel impulse response is obtained by introducing the elevation parameters to the $2 \mathrm{D}$ model in (4.2) as

$$
\mathbf{H}(t)=\sum_{p=1}^{P} \alpha_{p} \mathbf{a}_{\mathrm{r}}\left(\theta_{p}, \vartheta_{p}\right) \mathbf{a}_{\mathrm{t}}^{T}\left(\phi_{p}, \varphi_{p}\right) e^{j \omega_{p} t}
$$

where $\vartheta_{p}$ and $\varphi_{p}$ are the elevation angles of arrival and departure, respectively. Note that (4.108) is applicable to any array geometry, we will consider a typical scenario with a $N_{x} \times N_{y}$ UPA at the Base Station (BS) as shown in Fig. 4.2 and a M-element ULA at the Mobile Station (MS). Here, we assume that the BS antenna elements have equal spacings $d_{\mathrm{t}}^{\mathrm{x}}$ and $d_{\mathrm{t}}^{\mathrm{y}}$ along the $x$-axis and $y$-axis, respectively and that the MS antenna are spaced $d_{\mathrm{r}}$ apart. The channel impulse response between the $\left(n_{x}, n_{y}\right)$ th BS antenna and $m$ th MS antenna can therefore be expressed as

$$
\begin{aligned}
h_{\left(n_{x}, n_{y}\right), m}(t)= & \sum_{p=1}^{P} \alpha_{p} e^{j\left\{2 \pi \left[\left(n_{x}-1\right) d_{\mathrm{t}}^{\mathrm{x}} \cos \phi_{p} \sin \varphi_{p}+\left(n_{y}-1\right) d_{\mathrm{t}}^{\mathrm{y}} \cos \phi_{p} \cos \varphi_{p}\right.\right.} \\
& \left.\left.+(m-1) d_{\mathrm{r}} \cos \theta_{p} \sin \vartheta_{p}\right]+\omega_{p} t\right\}
\end{aligned}
$$

Assuming that the MS moves in the horizontal plane with velocity $v$ at an angle of $\vartheta_{\mathrm{v}}$ relative to the array broadside, the Doppler frequency of the $p$ th path can be shown to be

$$
\omega_{p}=\frac{2 \pi v\left(\cos \vartheta_{\mathrm{v}} \cos \theta_{p} \cos \vartheta_{p}+\sin \vartheta_{\mathrm{v}} \cos \theta_{p} \sin \vartheta_{p}\right)}{\lambda}
$$

\footnotetext{
${ }^{9} \mathrm{~A}$ similar procedure can be used to extend the other methods to $3 \mathrm{D}$ channels.
} 


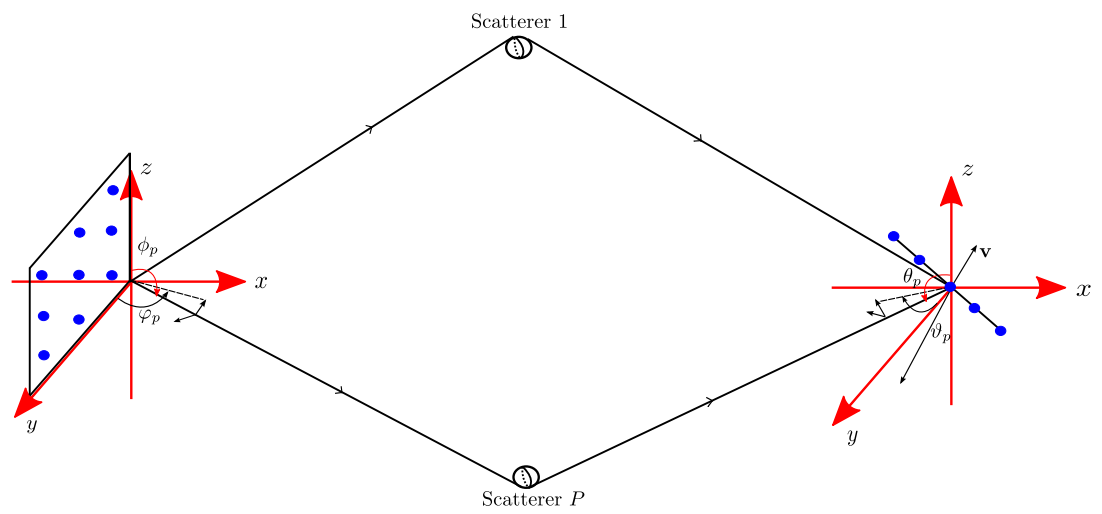

Figure 4.2: Illustration of 3D MIMO propagation with UPA at the BS and ULA at MS.

We arrange (4.109) into a $N_{x} N_{y} \times M$ matrix

$$
\mathbf{H}(t)=\left[\begin{array}{ccccccc}
h_{(1,1), 1} & h_{(1,2), 1} & \cdots & h_{\left(1, N_{y}\right), 1} & h_{(2,1), 1} & \cdots & h_{\left(N_{x}, N_{y}\right), 1} \\
h_{(1,1), 2} & h_{(1,2), 2} & \cdots & h_{\left(1, N_{y}\right), 2} & h_{(2,1), 2} & \cdots & h_{\left(N_{x}, N_{y}\right), 2} \\
\vdots & \vdots & \ddots & \vdots & \vdots & \ddots & \vdots \\
h_{(1,1), M} & h_{(1,2), M} & \cdots & h_{\left(1, N_{y}\right), M} & h_{(2,1), M} & \cdots & h_{\left(N_{x}, N_{y}\right), M}
\end{array}\right]
$$

which can be expressed in the form of (4.108) with the array response vectors defined as

$$
\mathbf{a}_{\mathbf{r}}\left(\theta_{p}, \vartheta_{p}\right)=\left[\begin{array}{c}
1 \\
e^{\left\{-j 2 \pi d_{\mathrm{r}} \cos \theta_{p} \sin \vartheta_{p}\right\}} \\
e^{\left\{-j 4 \pi d_{\mathrm{r}} \cos \theta_{p} \sin \vartheta_{p}\right\}} \\
\vdots \\
e^{\left\{-j(M-1) \pi_{\mathrm{r}} \cos \theta_{p} \sin \vartheta_{p}\right\}}
\end{array}\right]
$$

and

$$
\mathbf{a}_{\mathrm{t}}\left(\phi_{p}, \varphi_{p}\right)=\mathbf{a}_{\mathrm{t}}^{\mathrm{x}}\left(\phi_{p}, \varphi_{p}\right) \otimes \mathbf{a}_{\mathrm{t}}^{\mathrm{y}}\left(\phi_{p}, \varphi_{p}\right)
$$


where $\mathbf{a}_{\mathrm{t}}^{\mathrm{x}}\left(\phi_{p}, \varphi_{p}\right)$ is defined analogous to (4.112) with $M$ replaced by $N_{x}$ and $d_{\mathrm{r}}$ by $d_{\mathrm{t}}^{\mathrm{x}}$. In $(4.113), \mathbf{a}_{\mathrm{t}}^{\mathrm{y}}\left(\phi_{p}, \varphi_{p}\right)$ is given by

$$
\mathbf{a}_{\mathrm{t}}^{\mathrm{y}}\left(\phi_{p}, \varphi_{p}\right)=\left[\begin{array}{c}
1 \\
e^{\left\{-j 2 \pi d_{\mathrm{t}}^{\mathrm{y}} \cos \phi_{p} \cos \varphi_{p}\right\}} \\
e^{\left\{-j 4 \pi d_{\mathrm{t}}^{\mathrm{y}} \cos \phi_{p} \cos \varphi_{p}\right\}} \\
\vdots \\
e^{\left\{-j\left(N_{y}-1\right) \pi d_{\mathrm{t}}^{\mathrm{y}} \cos \phi_{p} \cos \varphi_{p}\right\}}
\end{array}\right]
$$

Note that the $3 \mathrm{D}$ channel model can be parametrized by $\left\{\alpha_{p}, \theta_{p}, \vartheta_{p}, \phi_{p}, \varphi_{p}\right\}_{p=1}^{P}$ which needs to be estimated. Again, we assume that the parameters and the mobile speed remain constant over the region considered. The sampled 3D impulse response at the $k$ instant can be represented as

$$
\mathbf{H}(k)=\sum_{p=1}^{P} \alpha_{p} \mathbf{a}_{\mathrm{r}}\left(\theta_{p}, \vartheta_{p}\right) \mathbf{a}_{\mathrm{t}}^{T}\left(\phi_{p}, \varphi_{p}\right) e^{j k \nu_{p}}
$$

\subsubsection{D Prediction Based on DOD/DOA-MEMCHAP}

We will now describe the 3D extension of the DOD/DOA-MEMCHAP. As in the $2 \mathrm{D}$ schemes, there are two key problems. First, the number of antenna elements at both ends of the link are less than the number of paths. Second, estimation of azimuth and elevation parameters using the ULA at the MS often results in ambiguity in one of the dimensions. Our algorithm increases the number of resolvable paths by performing a transformation on the available 3D CSI. The temporal structure of the channel is used as an additional dimension at the MS which allows for the extraction of both azimuth and elevation angles of arrival as well as the Doppler frequencies ${ }^{10}$.

\footnotetext{
${ }^{10}$ This is possible provided that the array elements are not aligned in the direction of motion. Since explicit knowledge of the AOAs and AODs are not required for the extrapolation, our methods still offer reasonable performance when the antenna elements are aligned in the direction of motion as illustrated in Fig. 4.3.
} 
For convenience, let us denote

$$
\begin{aligned}
\mu_{p}^{\mathrm{r}} & =2 \pi d_{\mathrm{r}}^{\mathrm{x}} \cos \theta_{p} \sin \vartheta_{p} \\
\mu_{p}^{\mathrm{tx}} & =2 \pi d_{\mathrm{t}}^{\mathrm{x}} \cos \phi_{p} \sin \varphi_{p} \\
\mu_{p}^{\mathrm{ty}} & =2 \pi d_{\mathrm{t}}^{\mathrm{y}} \cos \phi_{p} \cos \varphi_{p}
\end{aligned}
$$

Substituting (4.116) into (4.115) gives

$$
\mathbf{H}(k)=\sum_{p=1}^{P} \alpha_{p} \mathbf{a}_{\mathrm{r}}\left(\mu_{p}^{\mathrm{r}}\right) \mathbf{a}_{\mathrm{t}}^{T}\left(\mu_{p}^{\mathrm{tx}}, \mu_{p}^{\mathrm{ty}}\right) e^{j k \nu_{p}}
$$

Based on (4.117), the 3D channel prediction can be achieved by estimating the spatial frequencies $\left\{\mu_{p}^{\mathrm{r}}, \mu_{p}^{\mathrm{tx}}, \mu_{p}^{\mathrm{ty}}\right\}_{p=1}^{P}$, Doppler frequencies $\left\{\nu_{p}\right\}_{p=1}^{P}$ and complex amplitudes $\left\{\alpha_{p}\right\}_{p=1}^{P}$. The stages involved in the prediction will be discussed in the sequel.

\section{Data Transformation}

Given $K$ noisy estimates of the channel $\hat{\mathbf{H}}(k) ; k=0,1, \cdots, K-1$, we first perform a vectorization on each of the channel matrix samples, thus

$$
\begin{aligned}
\hat{\mathbf{h}}(k) & =\operatorname{vec}[\hat{\mathbf{H}}(k)] \\
& =\sum_{p=1}^{P} \alpha_{p}\left(\mathbf{a}_{\mathrm{r}}\left(\mu^{\mathrm{r}}-p\right) \otimes \mathbf{a}_{\mathrm{t}}\left(\mu_{p}^{\mathrm{tx}}, \mu_{p}^{\mathrm{ty}}\right) e^{j k \nu_{p}}+\mathbf{w}(k)\right.
\end{aligned}
$$

Assuming that the maximum possible number of paths $P_{\max }$ is known apriori, we select a free parameter $Z$ such that $P_{\max }+1 \leq N_{\mathrm{x}} N_{\mathrm{y}} M Z$ and form the Hankel data matrix

$$
\mathcal{D}=\left[\begin{array}{cccc}
\hat{\mathbf{h}}(0) & \hat{\mathbf{h}}(1) & \cdots & \hat{\mathbf{h}}(K-Z) \\
\hat{\mathbf{h}}(1) & \hat{\mathbf{h}}(2) & \cdots & \hat{\mathbf{h}}(K-Z+1) \\
\vdots & \vdots & \ddots & \vdots \\
\hat{\mathbf{h}}(Z-1) & \hat{\mathbf{h}}(Z) & \cdots & \hat{\mathbf{h}}(K-1)
\end{array}\right]
$$


Using (4.118), the data in each column of (4.119) can be shown to be

$$
\mathbf{d}(z)=\sum_{p=1}^{P} \beta_{p}(z)\left(\mathbf{a}_{\mathrm{r}}\left(\mu_{p}^{\mathrm{r}}\right) \otimes \mathbf{a}_{\mathrm{t}}\left(\mu_{p}^{\mathrm{tx}}, \mu_{p}^{\mathrm{ty}}\right) \otimes \mathbf{a}_{\mathrm{d}}\left(\nu_{p}\right)\right)+\mathbf{w}(z)
$$

where $\beta_{p}(z)=\alpha_{p} e^{(z-1) \nu_{p}}$ and $\mathbf{a}_{\mathrm{d}}\left(\nu_{p}\right)$ is the $Z \times 1$ vector

$$
\mathbf{a}_{\mathrm{d}}\left(\nu_{p}\right)=\left[\begin{array}{lllll}
1 & e^{j \nu_{p}} & e^{2 \nu_{p}} & \cdots & e^{(Z-1) \nu_{p}}
\end{array}\right]^{T}
$$

Equation (4.120) can be expressed in matrix form as

$$
\begin{aligned}
\mathbf{d}(z) & =\left(\mathbf{A}_{\mathrm{r}}\left(\boldsymbol{\mu}^{\mathrm{r}}\right) \diamond \mathbf{A}_{\mathrm{t}}\left(\boldsymbol{\mu}^{\mathrm{tx}}\right) \diamond \mathbf{A}_{\mathrm{t}}\left(\boldsymbol{\mu}^{\mathrm{ty}}\right) \diamond \mathbf{A}_{\mathrm{d}}(\boldsymbol{\nu})\right) \boldsymbol{\beta}(z)+\mathbf{w}(z) \\
& =\mathbf{A} \boldsymbol{\beta}(z)+\mathbf{w}(z)
\end{aligned}
$$

$\boldsymbol{\beta}(z)=\left[\begin{array}{llll}\beta_{1}(z) & \beta_{2}(z) & \cdots & \beta_{P}\end{array}\right]^{T}$ and $\mathbf{A}_{\mathrm{r}}\left(\boldsymbol{\mu}^{\mathrm{r}}\right)$ is defined in (4.26). $\mathbf{A}_{\mathrm{t}}\left(\boldsymbol{\mu}^{\mathrm{tx}}\right)$, $\mathbf{A}_{\mathrm{t}}\left(\boldsymbol{\mu}^{\mathrm{ty}}\right)$ and $\mathbf{A}_{\mathrm{d}}(\boldsymbol{\nu})$ are Vandermonde structured matrices defined as

$$
\begin{aligned}
\mathbf{A}_{\mathrm{t}}\left(\boldsymbol{\mu}^{\mathrm{tx}}\right) & =\left[\begin{array}{cccc}
1 & 1 & \cdots & 1 \\
e^{j \mu_{1}^{\mathrm{tx}}} & e^{j \mu_{2}^{\mathrm{tx}}} & \cdots & e^{j \mu_{P}^{\mathrm{tx}}} \\
\vdots & \vdots & \ddots & \vdots \\
e^{j\left(N_{x}-1\right) \mu_{1}^{\mathrm{tx}}} & e^{j\left(N_{x}-1\right) \mu_{2}^{\mathrm{tx}}} & \cdots & e^{j\left(N_{x}-1\right) \mu_{P}^{\mathrm{tx}}}
\end{array}\right] \\
\mathbf{A}_{\mathrm{t}}\left(\boldsymbol{\mu}^{\mathrm{ty}}\right) & =\left[\begin{array}{cccc}
1 & 1 & \cdots & 1 \\
e^{j \mu_{1}^{\mathrm{ty}}} & e^{j \mu_{2}^{\mathrm{ty}}} & \cdots & e^{j \mu_{P}^{\mathrm{ty}}} \\
\vdots & \vdots & \ddots & \vdots \\
e^{j\left(N_{y}-1\right) \mu_{1}^{\mathrm{ty}}} & e^{j\left(N_{y}-1\right) \mu_{2}^{\mathrm{ty}}} & \cdots & e^{j\left(N_{y}-1\right) \mu_{P}^{\mathrm{ty}}}
\end{array}\right]
\end{aligned}
$$

and

$$
\mathbf{A}_{\mathrm{d}}(\boldsymbol{\nu})=\left[\begin{array}{cccc}
1 & 1 & \cdots & 1 \\
e^{j \nu_{1}} & e^{j \nu_{2}} & \cdots & e^{j \nu_{P}} \\
\vdots & \vdots & \ddots & \vdots \\
e^{j(Z-1) \nu_{1}} & e^{j(Z-1) \nu_{2}} & \cdots & e^{j(Z-1) \nu_{P}}
\end{array}\right]
$$




\section{Joint Parameter Estimation}

We begin by estimating the spatio-temporal covariance matrix from (4.119), given by

$$
\hat{\mathbf{C}}=\frac{\mathcal{D D}^{H}}{K-Z+1}
$$

The number of dominant sources is then estimated using the MMDL as

$$
\hat{P}=\arg \min _{p \in[1, U]}\left[(K-Z+1) \log \left(\lambda_{p}\right)+\frac{1}{2}\left(p^{2}+p\right) \log (K-Z+1)\right]
$$

where $U=N_{\mathrm{x}} N_{\mathrm{y}} M Z$ and $\left\{\lambda_{p}\right\}_{p=1}^{U}$ are the ordered eigenvalues of $\hat{\mathbf{C}}$. Using $\hat{P}$, the eigen-decomposition of $\hat{\mathbf{C}}$ can be separated into the signal and noise subspaces as

$$
\begin{aligned}
\hat{\mathbf{C}} & =\left[\begin{array}{ll}
\mathbf{E}_{\mathrm{s}} & \mathbf{E}_{\mathrm{n}}
\end{array}\right]\left[\begin{array}{ll}
\Lambda_{\mathrm{s}} & \\
& \Lambda_{\mathrm{n}}
\end{array}\right]\left[\begin{array}{l}
\mathbf{E}_{\mathrm{s}}^{H} \\
\mathbf{E}_{\mathrm{n}}^{H}
\end{array}\right] \\
& =\mathbf{E}_{\mathrm{s}} \boldsymbol{\Lambda}_{\mathrm{s}} \mathbf{E}_{\mathrm{s}}^{H}+\mathbf{E}_{\mathrm{n}} \boldsymbol{\Lambda}_{\mathrm{n}} \mathbf{E}_{\mathrm{n}}^{H}
\end{aligned}
$$

where $\mathbf{E}_{\mathrm{s}} \in \mathbb{C}^{U \times \hat{P}}$ and $\boldsymbol{\Lambda}_{\mathrm{s}} \in \mathbb{R}^{U \times \hat{P}}$ are the signal subspace eigenvectors and the associated eigenvalues, respectively. $\quad \mathbf{E}_{\mathrm{n}} \in \mathbb{C}^{U \times(U-\hat{P})}$ and $\boldsymbol{\Lambda}_{\mathrm{n}} \in$ $\mathbb{R}^{(U-\hat{P}) \times(U-\hat{P})}$ corresponds to the noise eigenvectors and eigenvalues. Since, the data matrix in (4.119) exhibits translational invariance in four dimensions, the multidimensional extension of ESPRIT can be applied to jointly estimate the spatial frequencies in (4.116) and Doppler frequencies. Using (4.53), we form eight selection matrices (two for each dimension in the trans- 
formed data), specifically

$$
\begin{aligned}
& \mathbf{J}_{\mathrm{d} 1}=\mathbf{I}_{M} \otimes \mathbf{I}_{N_{\mathrm{x}}} \otimes \mathbf{I}_{N_{\mathrm{y}}} \otimes \mathbf{J}_{1 \mathrm{~d}} \\
& \mathbf{J}_{\mathrm{d} 2}=\mathbf{I}_{M} \otimes \mathbf{I}_{N_{\mathrm{x}}} \otimes \mathbf{I}_{N_{\mathrm{y}}} \otimes \mathbf{J}_{2 \mathrm{~d}} \\
& \mathbf{J}_{\mathrm{tx} 1}=\mathbf{I}_{M} \otimes \mathbf{J}_{1 \mathrm{tx}} \otimes \mathbf{I}_{N_{\mathrm{y}}} \otimes \mathbf{I}_{Z} \\
& \mathbf{J}_{\mathrm{tx} 2}=\mathbf{I}_{M} \otimes \mathbf{J}_{2 \mathrm{tx}} \otimes \mathbf{I}_{N_{\mathrm{y}}} \otimes \mathbf{I}_{Z} \\
& \mathbf{J}_{\mathrm{ty} 1}=\mathbf{I}_{M} \otimes \mathbf{I}_{N_{\mathrm{x}}} \otimes \mathbf{J}_{1 \mathrm{ty}} \otimes \mathbf{I}_{Z} \\
& \mathbf{J}_{\mathrm{ty} 2}=\mathbf{I}_{M} \otimes \mathbf{I}_{N_{\mathrm{x}}} \otimes \mathbf{J}_{2 \mathrm{ty}} \otimes \mathbf{I}_{Z} \\
& \mathbf{J}_{\mathrm{r} 1}=\mathbf{J}_{1 \mathrm{r}} \otimes \mathbf{I}_{N_{\mathrm{x}}} \otimes \mathbf{I}_{N_{\mathrm{y}}} \otimes \mathbf{I}_{Z} \\
& \mathbf{J}_{\mathrm{r} 2}=\mathbf{J}_{2 \mathrm{r}} \otimes \mathbf{I}_{N_{\mathrm{x}}} \otimes \mathbf{I}_{N_{\mathrm{y}}} \otimes \mathbf{I}_{Z}
\end{aligned}
$$

Using (4.129), we form the invariance equations

$$
\begin{gathered}
\mathbf{J}_{\mathrm{d} 1} \hat{\mathbf{E}}_{\mathrm{s}}=\mathbf{J}_{\mathrm{d} 2} \hat{\mathbf{E}}_{\mathrm{s}} \boldsymbol{\Phi}_{\mathrm{d}} \\
\mathbf{J}_{\mathrm{r} 1} \hat{\mathbf{E}}_{\mathrm{s}}=\mathbf{J}_{\mathrm{r} 2} \hat{\mathbf{E}}_{\mathrm{s}} \boldsymbol{\Phi}_{\mathrm{r}} \\
\mathbf{J}_{\mathrm{tx} 1} \hat{\mathbf{E}}_{\mathrm{s}}=\mathbf{J}_{\mathrm{tx} 2} \hat{\mathbf{E}}_{\mathrm{s}} \boldsymbol{\Phi}_{\mathrm{tx}} \\
\mathbf{J}_{\mathrm{ty} 1} \hat{\mathbf{E}}_{\mathrm{s}}=\mathbf{J}_{\mathrm{ty} 2} \hat{\mathbf{E}}_{\mathrm{s}} \boldsymbol{\Phi}_{\mathrm{ty}}
\end{gathered}
$$

where $\boldsymbol{\Phi}_{\mathrm{u}}$ satisfies the relation

$$
\operatorname{eig}\left[\Phi_{\mathrm{u}}\right]=\mathbf{D}\left(\boldsymbol{\mu}_{\mathrm{u}}\right)
$$

By minimizing the mean squared error in (4.130), we obtain

$$
\begin{aligned}
\boldsymbol{\Phi}_{\mathrm{r}} & =\left(\left(\mathbf{J}_{\mathrm{r} 1} \mathbf{E}_{s}\right)^{H}\left(\mathbf{J}_{\mathrm{r} 1} \mathbf{E}_{s}\right)\right)^{-1}\left(\mathbf{J}_{\mathrm{r} 1} \mathbf{E}_{s}\right)^{H}\left(\mathbf{J}_{\mathrm{r} 2} \mathbf{E}_{s}\right) \\
\boldsymbol{\Phi}_{\mathrm{tx}} & =\left(\left(\mathbf{J}_{\mathrm{tx} 1} \mathbf{E}_{s}\right)^{H}\left(\mathbf{J}_{\mathrm{tx} 1} \mathbf{E}_{s}\right)\right)^{-1}\left(\mathbf{J}_{\mathrm{tx} 1} \mathbf{E}_{s}\right)^{H}\left(\mathbf{J}_{\mathrm{tx} 2} \mathbf{E}_{s}\right) \\
\boldsymbol{\Phi}_{\mathrm{ty}} & =\left(\left(\mathbf{J}_{\mathrm{ty} 1} \mathbf{E}_{s}\right)^{H}\left(\mathbf{J}_{\mathrm{ty} 1} \mathbf{E}_{s}\right)\right)^{-1}\left(\mathbf{J}_{\mathrm{ty} 1} \mathbf{E}_{s}\right)^{H}\left(\mathbf{J}_{\mathrm{ty} 2} \mathbf{E}_{s}\right) \\
\boldsymbol{\Phi}_{\mathrm{d}} & =\left(\left(\mathbf{J}_{\mathrm{d} 1} \mathbf{E}_{s}\right)^{H}\left(\mathbf{J}_{\mathrm{d} 1} \mathbf{E}_{s}\right)\right)^{-1}\left(\mathbf{J}_{\mathrm{d} 1} \mathbf{E}_{s}\right)^{H}\left(\mathbf{J}_{\mathrm{d} 2} \mathbf{E}_{s}\right)
\end{aligned}
$$

which can be solved for the spatial frequencies in (4.116) and Doppler estimates, with an additional pairing stage to associate the parameter sets for 
each path. As in Section 4.4, we use the Mean Eigenvalue Decomposition (MEVD) to automatically pair the estimates. Let

$$
\begin{aligned}
\boldsymbol{\Phi} & =\boldsymbol{\Phi}_{\mathrm{r}}+\boldsymbol{\Phi}_{\mathrm{tx}}+\boldsymbol{\Phi}_{\mathrm{ty}}+\boldsymbol{\Phi}_{\mathrm{d}} \\
& =\mathbf{T}^{-1} \boldsymbol{\Lambda} \mathbf{T}
\end{aligned}
$$

Using (4.131) and (4.133) yields

$$
\begin{aligned}
\mathbf{D}\left(\boldsymbol{\mu}_{\mathrm{r}}\right) & =\mathbf{T} \boldsymbol{\Phi}_{\mathrm{r}} \mathbf{T}^{-1} \\
\mathbf{D}\left(\boldsymbol{\mu}_{\mathrm{t}}^{\mathrm{x}}\right) & =\mathbf{T} \boldsymbol{\Phi}_{\mathrm{tx}} \mathbf{T}^{-1} \\
\mathbf{D}\left(\boldsymbol{\mu}_{\mathrm{t}}^{\mathrm{y}}\right) & =\mathbf{T} \boldsymbol{\Phi}_{\mathrm{ty}} \mathbf{T}^{-1} \\
\mathbf{D}(\boldsymbol{\nu}) & =\mathbf{T} \boldsymbol{\Phi}_{\mathrm{d}} \mathbf{T}^{-1}
\end{aligned}
$$

Estimates of the parameters can now be obtained from (4.134) as

$$
\begin{aligned}
\hat{\boldsymbol{\mu}}^{\mathrm{r}} & =\arg \left[\operatorname{diag}\left\{\mathbf{D}\left(\boldsymbol{\mu}_{\mathrm{r}}\right)\right\}\right] \\
\hat{\boldsymbol{\mu}}^{\mathrm{tx}} & =\arg \left[\operatorname{diag}\left\{\mathbf{D}\left(\boldsymbol{\mu}_{\mathrm{r}}^{\mathrm{x}}\right)\right\}\right] \\
\hat{\boldsymbol{\mu}}^{\mathrm{ty}} & =\arg \left[\operatorname{diag}\left\{\mathbf{D}\left(\boldsymbol{\mu}_{\mathrm{r}}^{\mathrm{y}}\right)\right\}\right] \\
\hat{\boldsymbol{\nu}} & =\arg [\operatorname{diag}\{\mathbf{D}(\boldsymbol{\nu})\}]
\end{aligned}
$$

\section{Amplitude Estimation}

Following estimation of other channel parameters, the complex amplitudes can be estimated in a least square sense. Using the model in (4.117), the first entry of the channel matrix can be expressed as

$$
\hat{h}_{(1,1), 1}(k)=\sum_{p=1}^{P} \alpha_{p} e^{j k \nu_{p}}+w(k) ; \quad k=0,1, \cdots, K-1
$$

Putting the $K$ equation in (4.136) into a matrix form yields

$$
\hat{\mathbf{h}}_{(1,1), 1}=\mathbf{F} \boldsymbol{\alpha}+\mathbf{w}
$$

where $\hat{\mathbf{h}}_{(1,1), 1}=\left[\hat{h}_{(1,1), 1}(0), \hat{h}_{(1,1), 1}(1), \cdots, \hat{h}_{(1,1), 1}(L-1)\right]^{T}, \boldsymbol{\alpha}=\left[\alpha_{1}, \alpha_{2}, \cdots, \alpha_{\hat{P}}\right]^{T}$ and $\mathbf{F}$ is the $L \times \hat{P}$ Vandermonde matrix defined analogously to (4.124) with 
$Z$ replaced by $K$ and $P$ with $\hat{P}$. Using (4.137), each entry of the channel matrix can be represented as

$$
\hat{\mathbf{h}}_{\left(n_{x}, n_{y}\right), m}=\mathbf{F}_{\left(n_{x}, n_{y}\right), m} \boldsymbol{\alpha}+\mathbf{w}
$$

where

$$
\mathbf{F}_{\left(n_{x}, n_{y}\right), m}=\mathbf{F} \diamond \mathbf{v}_{\left(n_{x}, n_{y}\right), m}
$$

with $\mathbf{v}_{\left(n_{x}, n_{y}\right), m}=\left[e^{\left\{\left(n_{x}-1\right) \mu_{1}^{\mathrm{tx}}+\left(n_{y}-1\right) \mu_{1}^{\mathrm{ty}}+(m-1) \mu_{1}^{\mathrm{r}}\right\}}, \cdots, e^{\left\{\left(n_{x}-1\right) \mu_{\hat{P}}^{\mathrm{tx}}+\left(n_{y}-1\right) \mu_{\hat{P}}^{\mathrm{ty}}+(m-1) \mu_{\hat{P}}^{\mathrm{r}}\right\}}\right]$.

Now assume that the $N_{x} N_{y} M$ equations in (4.139) are combined as

$$
\hat{\mathbf{h}}=\left[\hat{\mathbf{h}}_{(1,1), 1}, \hat{\mathbf{h}}_{(1,1), 2}, \cdots, \hat{\mathbf{h}}_{(1,1), M}, \hat{\mathbf{h}}_{(1,2), 1}, \cdots, \hat{\mathbf{h}}_{(1,2), M}, \cdots, \hat{\mathbf{h}}_{\left(N_{x}, N_{y}\right), M}\right]^{T}
$$

Using (4.139), (4.140) can be represented as

$$
\begin{aligned}
\hat{\mathbf{h}} & =(\mathbf{F} \diamond \mathbf{V}) \boldsymbol{\alpha}+\mathbf{w} \\
& =\mathbf{G} \boldsymbol{\alpha}+\mathbf{w}
\end{aligned}
$$

where $\mathbf{V}=\mathbf{A}_{\mathrm{r}} \diamond \mathbf{A}_{\mathrm{tx}} \diamond \mathbf{A}_{\mathrm{ty}}$. The solution of (4.141) via MSE minimization yields estimate of the complex amplitudes as

$$
\hat{\boldsymbol{\alpha}}=\left(\mathbf{G}^{H} \mathbf{G}+\eta \mathbf{I}\right)^{-1} \mathbf{G} \hat{\mathbf{h}}
$$

\section{Channel Prediction}

Based on the estimated parameters, the predicted CSI at time instant $k$ is computed using

$$
\tilde{h}_{\left(n_{x}, n_{y}\right), m}(k)=\sum_{p=1}^{\hat{P}} \hat{\alpha}_{p} e^{j\left\{(m-1) \hat{\mu}_{p}^{\mathrm{r}}+\left(n_{x}-1\right) \hat{\mu}_{p}^{\mathrm{tx}}+\left(n_{y}-1\right) \hat{\mu}_{p}^{\mathrm{ty}}+k \hat{\nu}_{p}\right\}}
$$

\subsection{Simulation and Results}

In this section we present simulation results to illustrate the performance of the proposed schemes along with a comparison of the computational complexity of the different algorithms. 
Table 4.4: Narrowband MIMO Channel Simulation Parameters

\begin{tabular}{ll}
\hline Parameter & value \\
\hline Carrier Frequency & $2.1 \mathrm{GHz}$ \\
Mobile Velocity & $50 \mathrm{kph}$ \\
Tx/Rx Antenna Conf. & ULA @ $1 / 2 \lambda$ spacing \\
Sample Density & $10 / \lambda$ \\
Taps & 1 \\
Training Length & 50,100 \\
\hline
\end{tabular}

\subsubsection{Performance Comparison}

We consider a $2 \times 2$ narrowband MIMO system with the simulation parameters listed in Table 4.4. Without loss of generality, we consider the first cluster (tap) ${ }^{11}$ in the WINNER II Urban macro-cell Non-Line-of-Sight (NLOS) channel model with all parameters other than those specified in Table 4.4 retaining their default values. Figures 4.4-4.5 show the effect of the amplitude estimation accuracy on the overall prediction performance. We compare the performance of the DOD/DOA-MEMCHAP scheme with the complex amplitudes estimated using only the first entry of the CSI as in (4.102) and using all entries of the CSI as in (4.106). In Fig. 4.4, we plot the Normalized Mean Squared Error (NMSE) versus the prediction horizon (in wavelengths). The negative values of prediction horizon values correspond to the training segment. We observe that the method using only a single entry of the CSI for amplitude estimation performs poorly when compared to the method utilizing all entries. This is intuitively obvious since increased data is expected to result in improved estimation performance. Figure 4.5 presents

\footnotetext{
${ }^{11}$ The schemes can be applied to all other clusters or to a summation of the impulse responses of all the clusters weighted according to their respective PDP. Our aim in this section is to compare the performance of the schemes and evaluate the effects of the spatial correlations on the different schemes.
} 
the Cumulative Distribution Function (CDF) of Normalized Squared Error (NSE) for a prediction horizon of $1 \lambda$. It shows that both single entry and all entries amplitude estimation based prediction exhibit similar performance for most of the channel realizations, except for a few instances where the single entry based approach have very high NMSE.

Figure 4.6 shows the NMSE versus prediction horizon for all methods. We observe that the DOD/DOA approach outperforms all other methods, with the RSSM and TSSM having approximately equal performance. The MSSM shows the highest NMSE over the entire range considered indicating that the utilization of the spatial structure for array gain in the DOD/DOA, TSSM and RSSM improves prediction performance. The similarity in performance of the TSSM and RSSM methods is expected since the numbers of transmit and receive antenna elements are equal in the simulated system. An increase in the number of elements at either ends is expected to improve the performance of the corresponding method. The corresponding CDF of NSE is presented in Fig. 4.7 at a prediction interval of $1 \lambda$. The DOD/DOA method shows the lowest NSE for all channel realizations followed by the TSSM and the RSSM methods.

The effects of sample density on the performance of the proposed methods is illustrated in Fig. 4.8 where we plot the NMSE versus prediction horizon. We consider a training segment of $1 \lambda$ with 50 and 100 samples corresponding to a sampling rate of $50 / \lambda$ and $100 / \lambda$, respectively. Unlike the result in Fig. 4.6, the MSSM method offers better performance in the training segment. However, the performance in the prediction segment remains the poorest. The corresponding NSE CDF at a prediction interval of $0.1 \lambda$ is shown in Fig. 4.9.

We show the effects of increasing SNR on the performance of the proposed methods in Fig. 4.10 where we plot the NMSE versus SNR for a prediction horizon of $1 \lambda$. As expected, as a consequence of improved parameter estimation accuracy, the NMSE of the algorithms decreases with increasing SNR. A comparison of the performance with the Cramer-Rao bound will be presented 
in later chapters.

Finally, we illustrate the performance of the 3D DOD/DOA-MEMCHAP scheme in Figs. 4.11-4.12, where we plot the NMSE versus SNR for different numbers of transmit and receive antennas. The channel is generated synthetically using the simulation parameters in Table 4.4. The amplitudes are generated as complex Gaussian distributed random variables, $\alpha_{p} \sim \mathcal{C N}(0,1)$. The azimuth angles of arrival and departure are assumed to be uniformly distributed, i.e., $\theta_{p}, \phi_{p} \sim \mathcal{U}[-\pi, \pi)$. The arrival and departure angles in the elevation are drawn from a Laplacian distribution with $\{\mu=1.26, \sigma=0.16\}$ and $\{\mu=0.90, \sigma=0.20\}^{12}$, respectively. Fig. 4.11 shows the performance of the $3 \mathrm{D}$ prediction approach for a 2-element receive antenna array and different transmit array sizes at a prediction horizon of $1 \lambda$. As in the 2D methods, the NMSE decreases with increasing number of antennas and/or SNR. In Fig. 4.12, we compare the performance of the 3D DOD/DOA-MEMCHAP and its $2 \mathrm{D}$ equivalent. We observe that while the $3 \mathrm{D}$ scheme with a $2 \times 2$ UPA at the BS outperforms the 2D approach with a 2-element ULA at the $\mathrm{BS}$, the 2D method performs better with 4-element ULA at the base station. This is expected since more parameters are estimated in the 3D method using the same amount of observation. It may therefore be advantageous to deploy a 4-element ULA at the BS instead of a $2 \times 2$ UPA in order to exploit the improvement in prediction performance. However, considerations has to be given to the benefits offered by 3D propagation using the UPA and the slight difference in the complexity of the parameter estimation stages of the two configurations.

\subsubsection{Complexity Analysis}

The major computational requirement of all the proposed methods (i.e. MSSM, TSSM, RSSM and DOD/DOA) is accounted for by the covariance

\footnotetext{
${ }^{12}$ These values are selected from the WINNER + specifications for urban macro scenarios $[6]$.
} 
matrix estimation, estimation of number of sources, ESPRIT based parameter estimation and complex weight estimation stages. The computation of the covariance matrices in the MSSM, TSSM, RSSM and DOD/DOA based methods has complexity $\mathcal{O}\left(N M T_{\mathrm{m}} R_{\mathrm{m}}^{2}\right), \mathcal{O}\left(N M^{2} T_{\mathrm{t}} R_{\mathrm{t}}^{2}\right), \mathcal{O}\left(N^{2} M T_{\mathrm{r}} R_{\mathrm{r}}^{2}\right)$ and $\mathcal{O}\left(N^{2} M^{2} T_{\mathrm{d}} R_{\mathrm{d}}^{2}\right)$, respectively. In order to allow for fair comparison of the algorithms, we choose the Hankel size parameters $R_{\mathrm{m}}, R_{\mathrm{t}}, R_{\mathrm{r}}$ and $R_{\mathrm{d}}$ such that the covariance matrices in (4.54), (4.70), (4.88) and (4.95) have the same dimension. Thus, the complexity for estimating the covariance matrix in each of the schemes has same order of magnitude. Similarly, the MMDL based estimation of number of paths has a principal complexity (accounted for by the eigendecomposition of the covariance matrix) of same order of magnitude for all methods. The ESPRIT and multidimensional ESPRIT based parameter estimation stages of the four algorithms only differ in the number of matrix multiplications and inversions required and has complexity $\mathcal{O}\left(R_{\mathrm{m}} \hat{P}^{2}+\hat{P}^{3}\right), \mathcal{O}\left(2 M R_{\mathrm{t}} \hat{P}^{2}+3 \hat{P}^{3}\right), \mathcal{O}\left(2 N R_{\mathrm{r}} \hat{P}^{2}+3 \hat{P}^{3}\right)$ and $\mathcal{O}\left(3 N M R_{\mathrm{d}} \hat{P}^{2}+4 \hat{P}^{3}\right)$ for the MSSM, TSSM, RSSM and DOD/DOA based approaches, respectively. Clearly, the complexity of the proposed schemes differ slightly for the parameter estimation stage. The DOD/DOA has the highest complexity followed by the TSSM and RSSM which has same computational requirement. ${ }^{13}$ The MSSM based method has the lowest computational complexity for the parameter estimation stage. Finally, all methods has the same complexity, $\mathcal{O}\left(N M K \hat{P}^{2}\right)$, for the weight estimation stage. A summary of the computational complexity of the proposed methods is presented in Table 4.5.

\subsection{Summary}

In this chapter, we proposed four different schemes for the prediction of narrowband MIMO channels based on parametric modeling. The proposed

\footnotetext{
${ }^{13}$ This assumes that the number of transmit and receive antenna elements are equal. If the system has higher number of receive antenna, the complexity of the TSSM would be greater than the RSSM and vice versa.
} 
Table 4.5: Complexity Comparison of the Proposed Prediction Schemes

\begin{tabular}{|c|c|c|c|c|}
\hline \multirow[t]{2}{*}{ Step } & \multicolumn{4}{|c|}{ Algorithm } \\
\hline & MSSM & TSSM & RSSM & $\mathrm{DOD} / \mathrm{DOA}$ \\
\hline Covariance Matrix Estimation & $\mathcal{O}\left(N M T_{\mathrm{m}} R_{\mathrm{m}}^{2}\right)$ & $\mathcal{O}\left(N M^{2} T_{\mathrm{t}} R_{\mathrm{t}}^{2}\right)$ & $\mathcal{O}\left(N^{2} M T_{\mathrm{r}} R_{\mathrm{r}}^{2}\right)$ & $\mathcal{O}\left(N^{2} M^{2} T_{\mathrm{d}} R_{\mathrm{d}}^{2}\right)$ \\
\hline Estimation of Number of Paths & $\mathcal{O}\left(R_{\mathrm{m}}^{3}\right)$ & $\mathcal{O}\left(M^{3} R_{\mathrm{t}}^{3}\right)$ & $\mathcal{O}\left(N^{3} R_{\mathrm{r}}^{3}\right)$ & $\mathcal{O}\left(N^{3} M^{3} R_{\mathrm{d}}^{3}\right)$ \\
\hline Parameter Estimation & $\mathcal{O}\left(R_{\mathrm{m}} \hat{P}^{2}+\hat{P}^{3}\right)$ & $\mathcal{O}\left(2 M R_{\mathrm{t}} \hat{P}^{2}+3 \hat{P}^{3}\right)$ & $\mathcal{O}\left(2 N R_{\mathrm{r}} \hat{P}^{2}+3 \hat{P}^{3}\right)$ & $\mathcal{O}\left(3 N M R_{\mathrm{d}} \hat{P}^{2}+4 \hat{P}^{3}\right)$ \\
\hline Weight Estimation & $\mathcal{O}\left(N M K \hat{P}^{2}\right)$ & $\mathcal{O}\left(N M K \hat{P}^{2}\right)$ & $\mathcal{O}\left(N M K \hat{P}^{2}\right)$ & $\mathcal{O}\left(N M K \hat{P}^{2}\right)$ \\
\hline
\end{tabular}

methods are based on different formulations of the double directional MIMO model and adaptation of multidimensional extension of ESPRIT for joint parameter extraction. We illustrated the prediction performance improvement offered by the spatial structure of the channel revealed by multiple sampling of the wavefield. We observed that the method utilizing both transmit and receive spatial structure for array gain outperform those using only receive (TSSM-MEMCHAP), only transmit (RSSM-MEMCHAP) and no spatial information (MSSM-MEMCHAP). A framework for extending the proposed methods to 3D propagation is also presented. The performance of the 3D method was evaluated using elevation parameters from the WINNER + channel model [6]. 


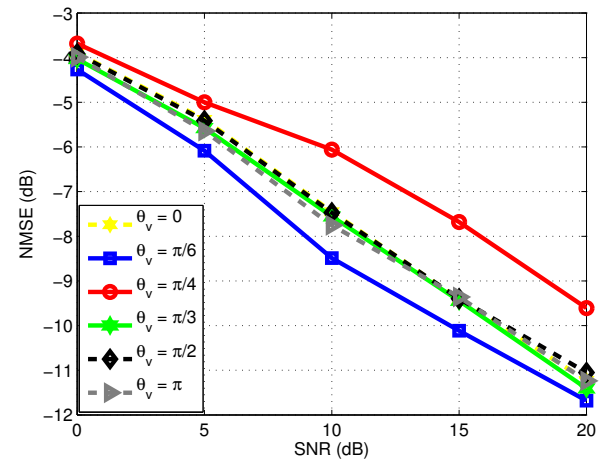

Figure 4.3: Effects of direction of motion on NMSE performance.

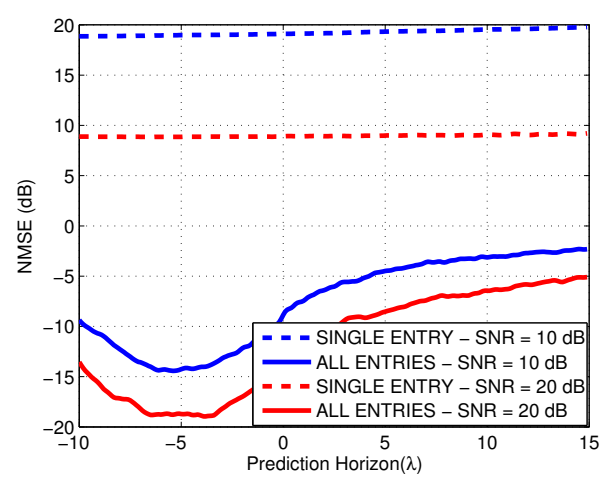

Figure 4.4: NMSE vs Horizon.

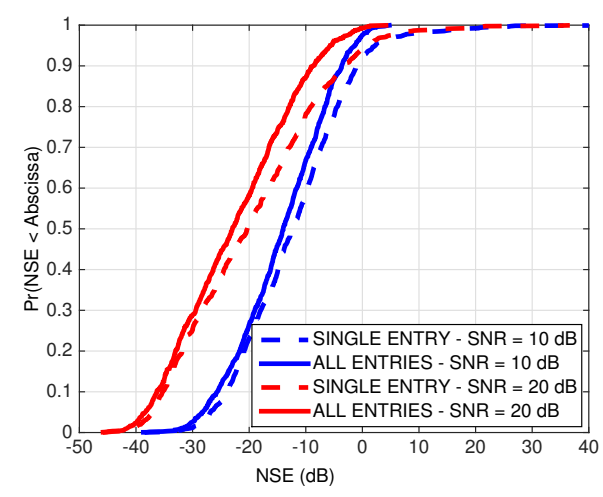

Figure 4.5: CDF of NSE for a $1 \lambda$ prediction.

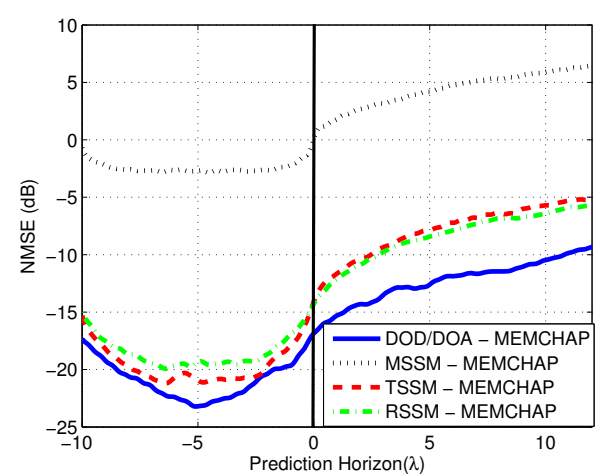

Figure 4.6: NMSE versus horizon for all methods.

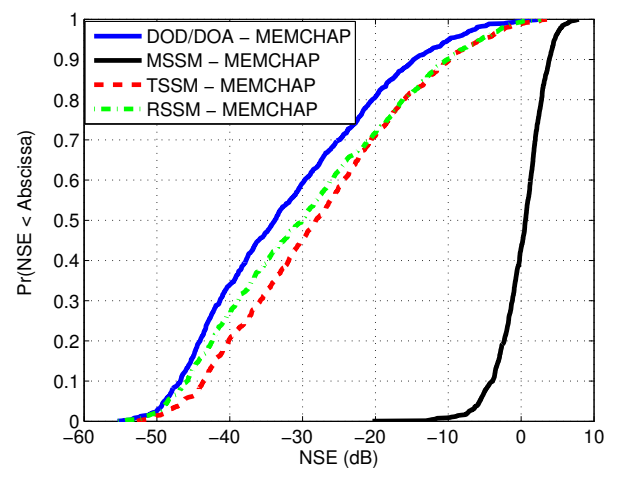

Figure 4.7: CDF of NSE for a horizon of $1 \lambda$.

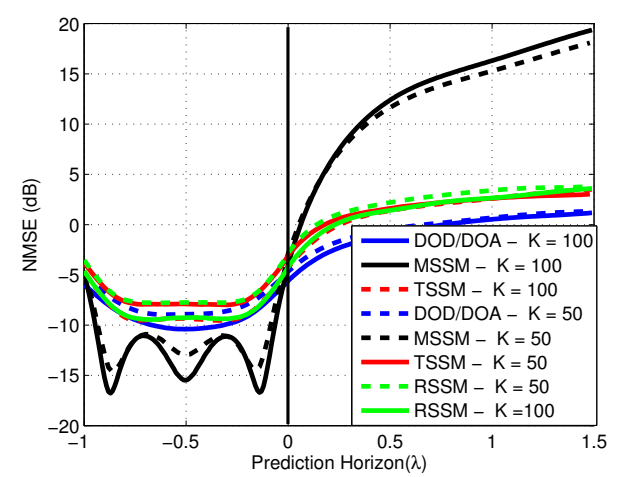

Figure 4.8: Effects of sampling rate on NMSE performance. 

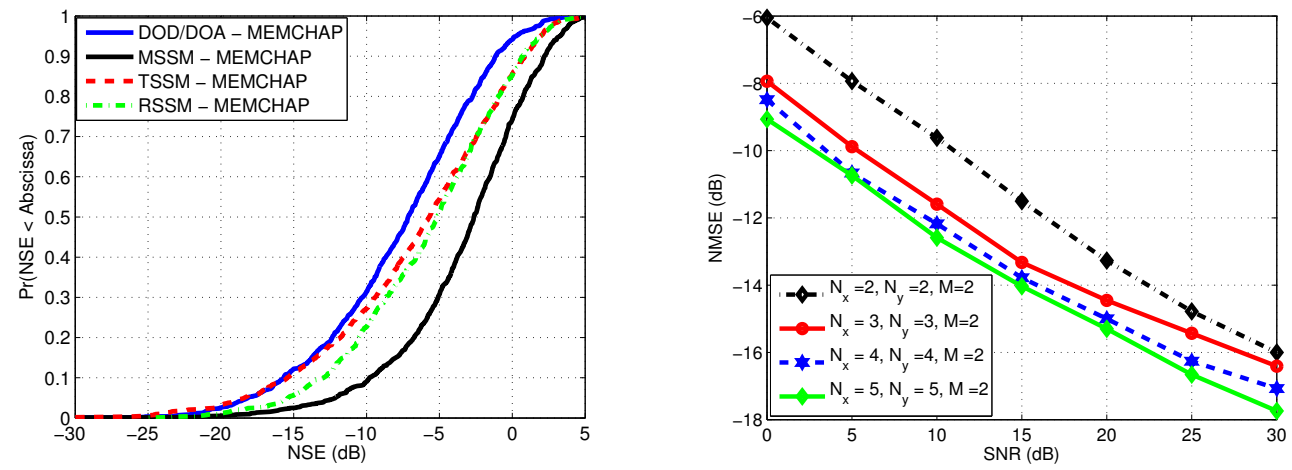

Figure 4.9: CDF of NSE for a horizon Figure 4.11: NMSE versus SNR at of $0.1 \lambda$. a horizon of $1 \lambda$ for $3 \mathrm{D} \mathrm{DOD} / \mathrm{DOA}$ MEMCHAP.
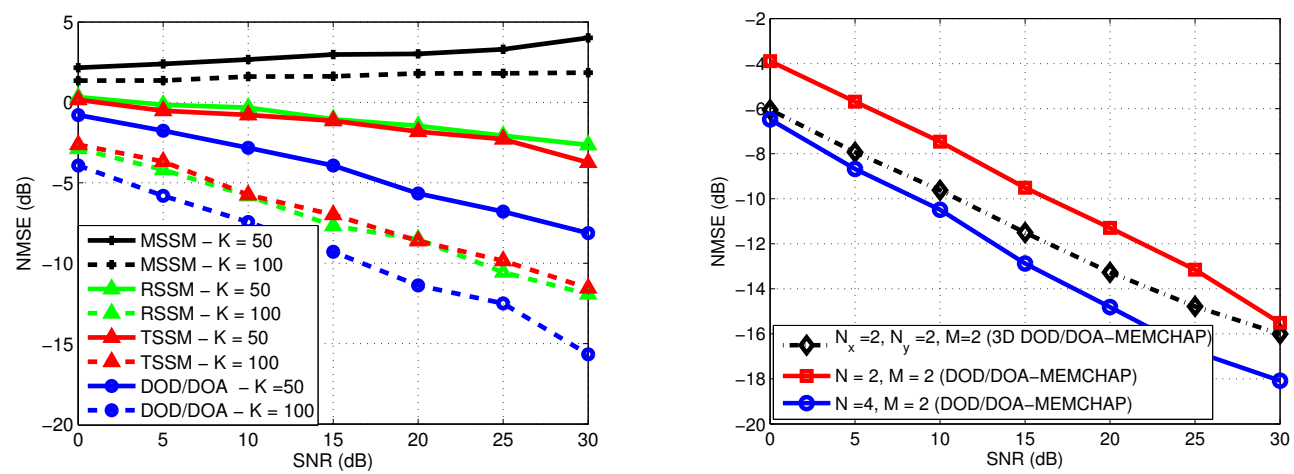

Figure 4.10: NMSE versus SNR at a Figure 4.12: NMSE versus SNR at a horizon of $1 \lambda$. horizon of $1 \lambda$. 


\section{5 Wideband MIMO Prediction}

T Chapter 4, we developed algorithms for the prediction of narrowband 1 MIMO channels. This chapter extends our prediction concept to wideband MIMO channels, where the resolution of the system allows for the extraction of path delays in addition to the AOA, AOD and Doppler frequencies. Motivated by the far-field cluster based double directional spatial channel modeling for MIMO systems as obtained in recent standardized MIMO channel models such as 3GPP/WINNER II [3] and COST273 [53], we also propose a 2 stage ESPRIT based parameter estimation algorithm. The scheme comprises of 1-dimensional cluster parameter estimation stage followed by 3-dimensional joint angle of arrival (AOA), angle of departure (AOD) and Doppler shift estimation. The predictors utilize the transmit spatial, frequency, receive spatial and temporal correlations of the channel to extract the parameters of the dominant clusters and apply these to predict future states of the channel. Estimation of the channel parameters offers a number of potential benefits viz:

- Reduction in the number of parameters to be estimated, 
- Increased number of resolvable rays with limited number of available samples, and

- Improved overall prediction performance.

The content of this chapter has been published in part in $[16,18]$.

\subsection{Channel Models}

We consider several formulations of a ray-based wideband spatial MIMO channel model for the development of the prediction schemes. The formulations are extensions of the continuous time impulse response of doubly selective SISO fading channels, defined as

$$
h(t ; \tau)=\sum_{p=1}^{P} \alpha_{p}(t) \delta\left(\tau-\tau_{p}(t)\right)
$$

where $t$ and $\tau$ are the time and delay variables respectively, $P$ is the number of paths, and $\alpha_{p}(t)$ and $\tau_{p}(t)$ are the time-varying complex attenuation and delay of the $p$ th path, respectively. We assume that the scattering sources are in the far field of both the transmit and receive antenna arrays such that the propagating waves can be modeled as plane waves. The complex attenuation of the $p$ th path can thus be defined as

$$
\alpha_{p}(t)=\sum_{r=1}^{R_{p}} \beta_{r, p} e^{j \omega_{r, p} t}
$$

where $R_{p}$ is the number of rays in the $p$ th path, $\omega_{r, p}$ is the radian Doppler frequency of the $r$ th ray in the pth path, $j=\sqrt{-1}$ and $\beta_{r, p}$ is the complex amplitude of the $r$ th ray in the $p$ th path. The model in (5.2) can be extended to a MIMO channel with $N$ transmit and $M$ receive antennas via the introduction of transmit and receive array structures, giving

$$
\mathbf{H}_{p}(t)=\sum_{r=1}^{R_{p}} \beta_{r, p} \mathbf{a}_{\mathrm{r}}\left(\theta_{r, p}\right) \mathbf{a}_{\mathrm{t}}^{T}\left(\phi_{r, p}\right) e^{j \omega_{r, p} t}
$$


Summing (5.3) over the clusters and taking the Fourier transform in the delay domain, we obtain the frequency response of the MIMO channel as

$$
\begin{aligned}
\mathbf{H}(t, f) & =\sum_{p=1}^{P} \mathbf{H}_{p}(t) e^{-j 2 \pi f \tau_{p}} \\
& =\sum_{p=1}^{P} \sum_{r=1}^{R_{p}} \beta_{r, p} \mathbf{a}_{\mathrm{r}}\left(\theta_{r, p}\right) \mathbf{a}_{\mathrm{t}}^{T}\left(\phi_{r, p}\right) e^{j \omega_{r, p} t-j 2 \pi f \tau_{p}}
\end{aligned}
$$

where $f$ is the frequency variable. Assuming symbol duration $T_{\text {samp }}$ and subcarrier spacing $\Delta f$, the sampled frequency response is given by

$$
\mathbf{H}(q, k)=\sum_{p=1}^{P} \sum_{r=1}^{R_{p}} \beta_{r, p} \mathbf{a}_{\mathrm{r}}\left(\theta_{r, p}\right) \mathbf{a}_{\mathrm{t}}^{T}\left(\phi_{r, p}\right) e^{j q \nu_{r, p}-j k \eta_{p}}
$$

where $q=0, \cdots, Q-1$ and $k=0, \cdots, K-1$ are the time and subcarrier indices, respectively. In (5.5) $\nu_{r, p}=\omega_{r, p} T_{\text {samp }}$ and $\eta_{p}=2 \pi \Delta f \tau_{p}$ are the normalized radian Doppler frequency and delay, respectively. Equation (5.5) will be referred to as the Cluster Based Model (CBM) and will form the basis for the development of the cluster based prediction method in Section 5.4. Combining indices $p$ and $r$ in (5.5), we obtain

$$
\mathbf{H}(q, k)=\sum_{z=1}^{Z} \beta_{z} \mathbf{a}_{\mathrm{r}}\left(\theta_{z}\right) \mathbf{a}_{\mathrm{t}}^{T}\left(\phi_{z}\right) e^{j q \nu_{z}-j k \eta_{z}}
$$

where $Z=\sum_{p=1}^{P} R_{p}$ is the total number of propagating rays. Each ray is characterized by the parameter set $\left\{\beta_{z}, \theta_{z}, \phi_{z}, \nu_{z}, \eta_{z}\right\}$. We assume that no two rays share a common parameter set, but different rays may have one or more equal parameters. Note that in practical scenarios, (5.6) has a finite support in the transmit angular, Doppler, delay and receive angular domains since the multipath parameters are bounded.

We now describe different formulations of the model in (5.6), where we progressively remove restrictions on the array structure. 


\subsubsection{DOD/DOA Model}

The first model is based on the assumption that the array response vectors $\mathbf{a}_{\mathrm{r}}$ and $\mathbf{a}_{\mathrm{t}}$ are explicit functions of the directions of arrival (DOA) and directions of departures (DOD) [110] as shown in (5.6). Similar to Section 4.1, we will consider systems with uniform linear arrays (ULA) at both ends of the link such that the transmit and receive steering vectors are given by (4.7) and (4.8), respectively.

\subsubsection{Transmit Spatial Signature Model (TSSM)}

Following a similar procedure as in Section 4.1.2, the TSSM equivalent of (5.6) can be expressed as

$$
\mathbf{H}(q, k)=\sum_{z=1}^{Z} \mathbf{a}_{\mathbf{r}}\left(\mu_{z}^{\mathrm{r}}\right) \mathbf{s}_{z}^{T} e^{j q \nu_{z}-j k \eta_{z}}
$$

\subsubsection{Matrix Spatial Signature Model (MSSM)}

Similar to the TSS model, the MSSM [110] replaces the product of the array steering vectors by an $N \times M$ unstructured matrix spatial signature, $\mathbf{S}$, giving

$$
\mathbf{H}(q, k)=\sum_{z=1}^{Z} \mathbf{S}_{z} e^{j q \nu_{z}-j k \eta_{z}}
$$

A summary of the number of parameters required for each model and dependence on number of antenna elements is shown in Table 5.1. We assume that $Q$ temporal samples and $K$ frequency samples of the channel frequency response matrix are available by transmitting known training sequences or from other channel estimation approaches. The estimated CSI matrix at time instant $q$ for the $k$ th subcarrier is defined as

$$
\hat{\mathbf{H}}(k, q)=\mathbf{H}(k, q)+\mathbf{N}(k, q)
$$

where $\mathbf{N}(k, q) \in \mathbb{C}^{N \times M}$ is a matrix of complex Gaussian random variables that accounts for channel estimation errors. 
Table 5.1: MIMO Model Parametrization and Dependence on Number of Antennas

\begin{tabular}{llll}
\hline Model & Structural Param. & Amp. & Real Param. \\
\hline DOD $/$ DOA & $\left\{\mu_{z}^{\mathrm{r}}, \mu_{z}^{\mathrm{t}}, \nu_{z}, \eta_{z}\right\}_{z=1}^{Z}$ & $\left\{\mathfrak{R}\left(\beta_{z}\right), \mathfrak{I}\left(\beta_{z}\right)\right\}_{z=1}^{Z}$ & $6 Z$ \\
TSSM & $\left\{\mu_{z}^{\mathrm{r}}, \nu_{z}, \eta_{z}\right\}_{z=1}^{Z}$ & $\left\{\mathfrak{R}\left(\mathbf{s}_{z}\right), \mathfrak{I}\left(\mathbf{s}_{z}\right)\right\}_{z=1}^{Z}$ & $Z(2 M+3)$ \\
MSSM & $\left\{\gamma_{z}, \eta_{z}\right\}_{z=1}^{Z}$ & $\left\{\mathfrak{R}\left(\mathbf{S}_{z}\right), \mathfrak{I}\left(\mathbf{S}_{z}\right)\right\}_{z=1}^{Z}$ & $2 Z(N M+1)$ \\
CBM & $\left\{\left\{\mu_{r, p}^{\mathrm{r}}, \mu_{r}^{\mathrm{t}}, \nu_{r, p}\right\}_{r=1}^{R_{p}}, \eta_{p}\right\}_{p=1}^{P}$ & $\left.\left\{\mathfrak{R}\left(\beta_{r, p}\right), \mathfrak{I}\left(\beta_{r, p}\right)\right\}_{r=1}^{R_{p}}\right\}_{p=1}^{P}$ & $5 Z+P$ \\
\hline
\end{tabular}

\subsection{Data Transformation}

Having described the channel model variations for the development of the prediction schemes, we now present the data preprocessing necessary for the extraction of the parameters from available channel observations.

\subsubsection{DOD/DOA Transformation}

As shown in (5.6), the DOD/DOA model is characterized by $4 Z$ structural parameters $\left\{\mu_{z}^{\mathrm{r}}, \mu_{z}^{\mathrm{t}}, \gamma_{z}, \eta_{z}\right\}_{z=1}^{Z}$ and $Z$ complex amplitudes. Extraction of these parameters from the channel observations requires a four dimensional array data structure. Let $\mathbf{h}(q, k)=\operatorname{vec}[\mathbf{H}(q, k)] \in \mathbb{C}^{N M \times 1}$ be a vector obtained by stacking the columns of $\mathbf{H}(q, k)$. Using (5.6) and the properties of the Kronecker product, it can be shown that

$$
\mathbf{h}(q, k)=\sum_{z=1}^{Z} \beta_{z}\left(\mathbf{a}_{\mathrm{r}}\left(\mu_{z}^{\mathrm{r}}\right) \otimes \mathbf{a}_{\mathrm{t}}\left(\mu_{z}^{\mathrm{t}}\right) e^{j q \nu_{z}-j k \eta_{z}}\right.
$$

Note that the transformation in (5.10) combines the receive and transmit spatial dimension of the channel. In order to introduce the temporal dimension, we define

$$
\mathbf{D}(k)=[\mathbf{h}(1, k) \quad \mathbf{h}(2, k) \quad \cdots \quad \mathbf{h}(Q, k)]
$$


and form a Hankel matrix by sliding an $N M \times R$ rectangular window through (5.11) to obtain

$$
\mathbf{D}_{k}=\left[\begin{array}{cccc}
\mathbf{h}(1, k) & \mathbf{h}(2, k) & \cdots & \mathbf{h}(R, k) \\
\mathbf{h}(2, k) & \mathbf{h}(3, k) & \cdots & \mathbf{h}(R+1, k) \\
\vdots & \vdots & \ddots & \vdots \\
\mathbf{h}(S, k) & \mathbf{h}(S+1, k) & \cdots & \mathbf{h}(Q, k)
\end{array}\right]
$$

where $S=Q-R+1$. The frequency dimension of the channel is similarly introduced by forming a block Hankel matrix from $K$ such matrices to obtain

$$
\mathbf{X}_{\mathrm{d}}=\left[\begin{array}{cccc}
\mathbf{D}_{1} & \mathbf{D}_{2} & \cdots & \mathbf{D}_{T} \\
\mathbf{D}_{2} & \mathbf{D}_{3} & \cdots & \mathbf{D}_{(T+1)} \\
\vdots & \vdots & \ddots & \vdots \\
\mathbf{D}_{U} & \mathbf{D}_{(U+1)} & \cdots & \mathbf{D}_{K}
\end{array}\right]
$$

where $U$ and $T$ are the Hankel matrix size parameters with $U=K-T+1$. The values of $S, R, T$, and $U$ are selected such that $N M S U \geq Z+1$. There is, however, a compromise in selecting these: large values of $S$ and $U$ increases the number of rows in $X_{\mathrm{d}}$ and hence the number of sources that can be resolved, but this results in small values of $R$ and $T$ which degrades the accuracy of covariance estimates. Using the model in (5.10) and the transformations in (5.12) and (5.13), the data in the columns of $\mathbf{X}_{\mathrm{d}}$ can be modeled as

$$
\mathbf{x}_{\mathrm{d}}(i)=\sum_{z=1}^{Z} \beta_{z} \mathbf{a}\left(\mu_{z}^{\mathrm{r}}, \mu_{z}^{\mathrm{t}}, \nu_{z}, \eta_{z}\right) e^{-j(i-1) \eta_{z}}
$$

where $\mathbf{a}\left(\mu_{z}^{\mathrm{r}}, \mu_{z}^{\mathrm{t}}, \nu_{z}, \eta_{z}\right)=\left(\mathbf{a}_{r}\left(\mu_{z}^{\mathrm{r}}\right) \otimes \mathbf{a}_{t}\left(\mu_{z}^{\mathrm{t}}\right) \otimes \mathbf{a}_{d}\left(\nu_{z}\right) \otimes \mathbf{a}_{\tau}\left(\eta_{z}\right)\right)$ with

$$
\begin{aligned}
& \mathbf{a}_{\mathrm{d}}\left(\nu_{z}\right)=\left[\begin{array}{llll}
1 & e^{j \nu_{z}} & \cdots & e^{j(R-1) \nu_{z}}
\end{array}\right]^{T} \\
& \mathbf{a}_{\mathbf{f}}\left(\eta_{z}\right)=\left[\begin{array}{llll}
1 & e^{-j \eta_{z}} & \cdots & e^{-j(U-1) \eta_{z}}
\end{array}\right]^{T}
\end{aligned}
$$

Defining $\alpha_{z}(i)=\beta_{z} e^{-j(i-1) \eta_{z}},(5.14)$ can be expressed as

$$
\begin{aligned}
\mathbf{x}_{\mathrm{d}}(i) & =\sum_{z=1}^{Z} \alpha_{z}(i) \mathbf{a}\left(\mu_{z}^{\mathrm{r}}, \mu_{z}^{\mathrm{t}}, \nu_{z}, \eta_{z}\right) \\
& =\mathbf{A}\left(\boldsymbol{\mu}^{\mathrm{r}}, \boldsymbol{\mu}^{\mathrm{t}}, \boldsymbol{\nu}, \boldsymbol{\eta}\right) \boldsymbol{\alpha}(i)
\end{aligned}
$$


Table 5.2: Wideband MIMO Data Domain and Parameters

\begin{tabular}{llllr}
\hline Model & \multicolumn{4}{c}{ Data Domain } \\
\cline { 2 - 5 } & Receive & Transmit & Temporal & Frequency \\
\hline DOA/DOD & AOA & AOD & Doppler Shift & Delay \\
TSSM & AOA & - & Doppler shift & Delay \\
MSSM & - & - & Doppler shift & Delay \\
\hline
\end{tabular}

where $\boldsymbol{\alpha}(i)=\left[\begin{array}{lll}\alpha_{1} & \cdots & \alpha_{Z}\end{array}\right] \in \mathbb{C}^{Z \times 1}$ and $\mathbf{A}=\left[\mathbf{a}\left(\mu_{1}^{\mathrm{r}}, \mu_{1}^{\mathrm{t}}, \nu_{1}, \eta_{1}\right) \quad \cdots\right.$ $\left.\mathbf{a}\left(\mu_{Z}^{\mathrm{r}}, \mu_{Z}^{\mathrm{t}}, \nu_{Z}, \eta_{Z}\right)\right]$ is a Vandermonde structured steering matrix, with $\boldsymbol{\mu}^{\mathrm{r}}, \boldsymbol{\mu}^{\mathrm{t}}, \boldsymbol{\nu}$ and $\boldsymbol{\eta}$ defined as $Z \times 1$ vectors of their respective parameters. It can be seen that (5.14) corresponds to a four dimensional array data model obtained by combining the transmit spatial, temporal, frequency and receive spatial dimensions of the wideband MIMO channel. A summary of the dimensions considered by each model and the corresponding parameters is shown in Table 5.2.

\subsubsection{TSS Transformation}

As shown in (5.8) and Table 5.1, parameterizing the TSSM requires $3 Z$ structural parameters $\left\{\mu_{z}^{\mathrm{r}}, \gamma_{z}, \eta_{z}\right\}_{z=1}^{Z}$. We will here derive a data structure that allows joint extraction of these parameters from (5.7). Using the $Q$ temporal samples, we start by forming a block Hankel matrix for each frequency sample,

$$
\mathbf{B}_{k}=\left[\begin{array}{cccc}
\mathbf{H}(1, k) & \mathbf{H}(2, k) & \cdots & \mathbf{H}(R, k) \\
\mathbf{H}(2, k) & \mathbf{H}(3, k) & \cdots & \mathbf{H}(R+1, k) \\
\vdots & \vdots & \ddots & \vdots \\
\mathbf{H}(S, k) & \mathbf{H}(S+1, k) & \cdots & \mathbf{H}(Q, k)
\end{array}\right]
$$

Note that (5.17) combines the receive spatial and temporal property of the channel into one dimension corresponding to the columns of $\mathbf{B}_{k}$. In order to include the frequency dimension of the wideband channel, we form another 
block Hankel matrix from (5.17) as

$$
\mathbf{X}_{\mathrm{t}}=\left[\begin{array}{cccc}
\mathbf{B}_{1} & \mathbf{B}_{2} & \cdots & \mathbf{B}_{T} \\
\mathbf{B}_{2} & \mathbf{B}_{3} & \cdots & \mathbf{B}_{T+1} \\
\vdots & \vdots & \ddots & \vdots \\
\mathbf{B}_{U} & \mathbf{B}_{U+1} & \cdots & \mathbf{B}_{K}
\end{array}\right]
$$

Based on the transformations in (5.17) and (5.18), the data in the columns of $\mathbf{X}_{\mathrm{t}}$ is given by

$$
\mathbf{x}_{\mathrm{t}}(i)=\mathbf{A}\left(\boldsymbol{\mu}^{\mathrm{r}}, \boldsymbol{\nu}, \boldsymbol{\eta}\right) \boldsymbol{\alpha}(i)
$$

where $\mathbf{A}\left(\boldsymbol{\mu}^{\mathrm{r}}, \boldsymbol{\nu}, \boldsymbol{\eta}\right)$ is defined as. ${ }^{1}$

$$
\mathbf{A}\left(\boldsymbol{\mu}^{\mathrm{r}}, \boldsymbol{\nu}, \boldsymbol{\eta}\right)=\mathbf{A}_{\mathrm{r}}\left(\boldsymbol{\mu}^{\mathrm{r}}\right) \diamond \mathbf{A}_{\mathrm{d}}(\boldsymbol{\nu}) \diamond \mathbf{A}_{\mathrm{f}}(\boldsymbol{\eta})
$$

where $\mathbf{A}_{\mathrm{r}}$ and $\mathbf{A}_{\mathrm{d}}$ are the Vandermonde matrices in (4.27) and (4.29), respectively. $\mathbf{A}_{\mathrm{f}}$ is defined as

$$
\mathbf{A}_{\mathbf{f}}=\left[\begin{array}{cccc}
1 & 1 & \cdots & 1 \\
e^{j \eta_{1}} & e^{j \eta_{2}} & \cdots & e^{j \eta_{Z}} \\
\vdots & \vdots & \ddots & \vdots \\
e^{j\left(R_{\mathrm{d}}-1\right) \eta_{1}} & e^{j\left(R_{\mathrm{d}}-1\right) \eta_{2}} & \cdots & e^{j\left(R_{\mathrm{d}}-1\right) \eta_{Z}}
\end{array}\right]
$$

\subsubsection{MSS Transformation}

The MSSM is parametrized by $2 Z$ structural parameters and $N M Z$ complex amplitude parameters. Extraction of these parameters from the channel requires a two-dimensional datum. Similar to (5.11), we form a Hankel matrix

$$
\mathbf{C}_{k}=\left[\begin{array}{cccc}
\mathbf{h}^{T}(1, k) & \mathbf{h}^{T}(2, k) & \cdots & \mathbf{h}^{T}(R, k) \\
\mathbf{h}^{T}(2, k) & \mathbf{h}^{T}(3, k) & \cdots & \mathbf{h}^{T}(R+1, k) \\
\vdots & \vdots & \ddots & \vdots \\
\mathbf{h}^{T}(S, k) & \mathbf{h}^{T}(S+1, k) & \cdots & \mathbf{h}^{T}(Q, k)
\end{array}\right]
$$

\footnotetext{
${ }^{1}$ For simplicity of notation, we use a common variable for the array steering matrix in all three models. The precise definition is obvious from the context.
} 
Note that the columns of $\mathbf{C}_{k}$ correspond to $S$ temporal measurements of the channel and can be modeled as

$$
\mathbf{c}_{k}(i)=\sum_{z=1}^{Z} \alpha_{z}(i) \mathbf{a}_{d}\left(\nu_{z}\right)
$$

where $\mathbf{a}_{d}\left(\nu_{z}\right)$ is an $S$ dimensional vector defined in (5.15). The data in (5.23) provides information about the Doppler shifts of the channel. The frequency structure of the channel can be included by forming a block Hankel matrix $\mathbf{X}_{\mathrm{m}}$ analogous to $\mathbf{X}_{\mathrm{d}}$ with $\mathbf{D}_{k}$ replaced with $\mathbf{C}_{k}$. The columns of $\mathbf{X}_{\mathrm{m}}$ can be shown using (5.23) to be

$$
\begin{aligned}
\mathbf{x}_{\mathrm{m}}(i) & =\sum_{z=1}^{Z} \alpha_{z}(i)\left(\mathbf{a}_{d}\left(\nu_{z}\right) \otimes \mathbf{a}_{\tau}\left(\eta_{z}\right)\right) \\
& =\mathbf{A}(\boldsymbol{\nu}, \boldsymbol{\eta}) \boldsymbol{\alpha}(i)
\end{aligned}
$$

As shown in (5.24), $\mathbf{X}_{\mathrm{m}}$ corresponds to a two-dimensional datum obtained by combining the temporal and frequency structure of the channel. The Doppler shifts and delays of arrival can therefore be extracted jointly using appropriate parameter estimation algorithms.

\subsection{Non-Cluster Based Prediction Methods}

We now propose prediction algorithms using the models developed in Section 5.1 and the transformed data derived in Section 5.2. We will henceforth refer to the algorithms as Wideband-Multidimensional ESPRIT based MIMO CHAnnel Predictor (WIMEMCHAP), and use the acronyms for the models as prefixes to distinguish the schemes. For example, the algorithm based on TSSM will be called TSSM-WIMEMCHAP and so on. Note that although the algorithms are based on the same idea of parametric modeling, they differ in the model, dimension of parameter estimation and number of amplitude and structural parameters to be estimated. 


\subsubsection{DOD/DOA-WIMEMCHAP}

Consider the transformed data model in (5.12) and (5.14). Since the array steering matrix $\mathbf{A}$ is equivalent to a product of four Vandermonde matrices, the invariance structure in $\mathbf{A}$ can be utilized to estimate the parameters of the channel. Motivated by the accuracy and computational efficiency of the ESPRIT algorithm [158], we propose an adaptation of multidimensional extension of ESPRIT to jointly extract the parameter sets $\left\{\mu_{z}^{\mathrm{r}}, \mu_{z}^{\mathrm{t}}, \gamma_{z}, \eta_{z}\right\}_{z=1}^{Z}$ and apply the parameter estimates to extrapolate the channel. As in the narrowband schemes of Chapter 4, the prediction algorithm can be divided into the following stages:

- covariance matrix estimation,

- estimate of the number of paths and subspace decomposition,

- joint parameter estimation,

- channel extrapolation.

We will now describe the above stages of the algorithm.

\section{Covariance Matrix Estimation}

In the presence of estimation or measurement noise, the model in (5.14) becomes

$$
\hat{\mathbf{x}}_{\mathrm{d}}(i)=\mathbf{A}\left(\boldsymbol{\mu}^{\mathrm{r}}, \boldsymbol{\mu}^{\mathrm{t}}, \boldsymbol{\nu}, \boldsymbol{\eta}\right) \boldsymbol{\alpha}(i)+\mathbf{n}(i)
$$

where $\mathbf{n}(i)$ models the effects of $\mathbf{N}$ in (5.5). The covariance matrix (containing the spatial, temporal and frequency correlations) can be estimated from $(5.25)$ as

$$
\begin{aligned}
\hat{\mathbf{C}}_{\mathrm{d}} & =\frac{1}{R T} \sum_{i=1}^{R T} \hat{\mathbf{x}}_{d}(i) \hat{\mathbf{x}}_{d}(i)^{H} \\
& =\mathbf{A}\left(\boldsymbol{\mu}^{\mathrm{r}}, \boldsymbol{\mu}^{\mathrm{t}}, \boldsymbol{\nu}, \boldsymbol{\eta}\right) \mathbf{C}_{\alpha \alpha} \mathbf{A}\left(\boldsymbol{\mu}^{\mathrm{r}}, \boldsymbol{\mu}^{\mathrm{t}}, \boldsymbol{\nu}, \boldsymbol{\eta}\right)^{H}+\sigma^{2} \mathbf{I}
\end{aligned}
$$


where $\mathbf{C}_{\alpha \alpha}=\frac{1}{R T} \sum_{i=1}^{R T} \boldsymbol{\alpha} \boldsymbol{\alpha}^{H}$ is the covariance matrix of the amplitude parameters. $\hat{\mathbf{C}}_{\mathrm{d}}$ can be expressed in terms of $\hat{\mathbf{X}}_{\mathrm{d}}$ in (5.11) as

$$
\hat{\mathbf{C}}_{\mathrm{d}}=\frac{1}{R T} \hat{\mathbf{X}}_{\mathrm{d}} \hat{\mathbf{X}}_{\mathrm{d}}^{H}
$$

\section{Number of Paths Estimation and Subspace Decomposition}

Again, we utilize the MMDL [85] to estimate the number of paths as

$$
\hat{Z}=\arg \min _{z=1, \cdots, N M S U-1} R T \log \left(\lambda_{z}\right)+\frac{1}{2}\left(z^{2}+z\right) \log R T
$$

where $\lambda_{z}$ are the eigenvalues of $\hat{\mathbf{C}}_{\mathrm{d}}$. Once $\hat{Z}$ has been estimated, the eigenvalue decomposition of $\mathbf{C}_{\mathrm{d}}$ can be expressed as

$$
\begin{aligned}
\mathbf{C}_{\mathrm{d}} & =\left[\begin{array}{ll}
\mathbf{E}_{\mathrm{s}} & \mathbf{E}_{\mathrm{n}}
\end{array}\right]\left[\begin{array}{ll}
\boldsymbol{\Lambda}_{\mathrm{s}} & \\
& \boldsymbol{\Lambda}_{\mathrm{n}}
\end{array}\right]\left[\begin{array}{l}
\mathbf{E}_{\mathrm{s}}^{H} \\
\mathbf{E}_{\mathrm{n}}^{H}
\end{array}\right] \\
& =\mathbf{E}_{\mathrm{s}} \boldsymbol{\Lambda}_{\mathrm{s}} \mathbf{E}_{\mathrm{s}}^{H}+\mathbf{E}_{\mathrm{n}} \boldsymbol{\Lambda}_{\mathrm{n}} \mathbf{E}_{\mathrm{n}}^{H}
\end{aligned}
$$

where $\mathbf{E}_{\mathrm{s}}$ and $\boldsymbol{\Lambda}_{\mathrm{s}}$ are the signal subspace eigenvectors and the associated eigenvalues, respectively. The noise subspace eigenvectors and eigenvalues are contained in $\mathbf{E}_{\mathrm{n}}$ and $\boldsymbol{\Lambda}_{\mathrm{n}}$, respectively.

\section{Parameter Estimation}

We now outline the process of obtaining the parameter sets $\left\{\mu_{z}^{\mathrm{r}}, \mu_{z}^{\mathrm{t}}, \gamma_{z}, \eta_{z}\right\}_{z=1}^{\hat{Z}}$ in Table 5.1. In order to explore the invariance structure [158] in the Vandermonde structured space-time-frequency manifold matrix, A, we define the selection matrices $\left\{\mathbf{J}_{\mathrm{di}}, \mathbf{J}_{\mathrm{ri}}, \mathbf{J}_{\mathrm{ti}}, \mathbf{J}_{\mathrm{fi}}\right\}_{i=1}^{2}$ analogous to those in (4.129). Similar to (4.130), we form the invariance equations

$$
\begin{aligned}
\mathbf{J}_{\mathrm{r} 2} \mathbf{E}_{s} & =\mathbf{J}_{\mathrm{r} 1} \mathbf{E}_{s} \boldsymbol{\Phi}_{\mathrm{r}} \\
\mathbf{J}_{\mathrm{t} 2} \mathbf{E}_{s} & =\mathbf{J}_{\mathrm{t} 1} \mathbf{E}_{s} \boldsymbol{\Phi}_{\mathrm{t}} \\
\mathbf{J}_{\mathrm{d} 2} \mathbf{E}_{s} & =\mathbf{J}_{\mathrm{d} 1} \mathbf{E}_{s} \boldsymbol{\Phi}_{\mathrm{d}} \\
\mathbf{J}_{\mathrm{f} 2} \mathbf{E}_{s} & =\mathbf{J}_{\mathrm{f} 1} \mathbf{E}_{s} \boldsymbol{\Phi}_{\mathrm{f}}
\end{aligned}
$$


where $\boldsymbol{\Phi}_{\mathrm{r}}, \boldsymbol{\Phi}_{\mathrm{t}}, \boldsymbol{\Phi}_{\mathrm{d}}$ and $\boldsymbol{\Phi}_{\mathrm{f}}$ are defined analogously to (4.82). We minimize the squared error of the equations in (5.30) to obtain

$$
\begin{aligned}
& \boldsymbol{\Phi}_{\mathrm{r}}=\left(\left(\mathbf{J}_{\mathrm{r} 2} \mathbf{E}_{s}\right)^{H}\left(\mathbf{J}_{\mathrm{r} 2} \mathbf{E}_{s}\right)\right)^{-1}\left(\mathbf{J}_{\mathrm{r} 2} \mathbf{E}_{s}\right)^{H}\left(\mathbf{J}_{\mathrm{r} 1} \mathbf{E}_{s}\right) \\
& \boldsymbol{\Phi}_{\mathrm{t}}=\left(\left(\mathbf{J}_{\mathrm{t} 2} \mathbf{E}_{s}\right)^{H}\left(\mathbf{J}_{\mathrm{t} 2} \mathbf{E}_{s}\right)\right)^{-1}\left(\mathbf{J}_{\mathrm{t} 2} \mathbf{E}_{s}\right)^{H}\left(\mathbf{J}_{\mathrm{t} 1} \mathbf{E}_{s}\right) \\
& \boldsymbol{\Phi}_{\mathrm{d}}=\left(\left(\mathbf{J}_{\mathrm{d} 2} \mathbf{E}_{s}\right)^{H}\left(\mathbf{J}_{\mathrm{d} 2} \mathbf{E}_{s}\right)\right)^{-1}\left(\mathbf{J}_{\mathrm{d} 2} \mathbf{E}_{s}\right)^{H}\left(\mathbf{J}_{\mathrm{d} 1} \mathbf{E}_{s}\right) \\
& \boldsymbol{\Phi}_{\mathrm{f}}=\left(\left(\mathbf{J}_{\mathrm{f} 2} \mathbf{E}_{s}\right)^{H}\left(\mathbf{J}_{\mathrm{f} 2} \mathbf{E}_{s}\right)\right)^{-1}\left(\mathbf{J}_{\mathrm{f} 2} \mathbf{E}_{s}\right)^{H}\left(\mathbf{J}_{\mathrm{f} 1} \mathbf{E}_{s}\right)
\end{aligned}
$$

Estimates of the AOAs, AODs, Doppler shifts and delays could be obtained directly from the solutions of (5.31)-(5.34) followed by an additional pairing stage. In order to achieve automatic pairing of the estimates, we utilize the MEVD [141]. Defining

$$
\Upsilon=\Phi_{\mathrm{r}}+\boldsymbol{\Phi}_{\mathrm{t}}+\boldsymbol{\Phi}_{\mathrm{d}}+\boldsymbol{\Phi}_{\mathrm{f}}
$$

we perform eigenvalue decomposition of $\Upsilon$ to obtain the common eigenvectors of the four matrices in the sum

$$
\Upsilon=\Sigma \Lambda \Sigma^{-1}
$$

The diagonal eigenvalue matrices are then obtained using

$$
\begin{aligned}
& \boldsymbol{\Xi}_{\mathrm{r}}=\boldsymbol{\Sigma}^{-1} \boldsymbol{\Phi}_{\mathrm{r}} \boldsymbol{\Sigma} \\
& \boldsymbol{\Xi}_{\mathrm{t}}=\boldsymbol{\Sigma}^{-1} \boldsymbol{\Phi}_{\mathrm{t}} \boldsymbol{\Sigma} \\
& \boldsymbol{\Xi}_{\mathrm{d}}=\boldsymbol{\Sigma}^{-1} \boldsymbol{\Phi}_{\mathrm{d}} \boldsymbol{\Sigma} \\
& \boldsymbol{\Xi}_{\mathrm{f}}=\boldsymbol{\Sigma}^{-1} \boldsymbol{\Phi}_{\mathrm{f}} \boldsymbol{\Sigma}
\end{aligned}
$$

where $\boldsymbol{\Xi}_{\mathrm{r}}=\operatorname{eig}\left(\boldsymbol{\Phi}_{\mathrm{r}}\right), \boldsymbol{\Xi}_{\mathrm{t}}=\operatorname{eig}\left(\boldsymbol{\Phi}_{\mathrm{t}}\right), \boldsymbol{\Xi}_{\mathrm{d}}=\operatorname{eig}\left(\boldsymbol{\Phi}_{\mathrm{d}}\right)$ and $\boldsymbol{\Xi}_{\mathrm{f}}=\operatorname{eig}\left(\boldsymbol{\Phi}_{\mathrm{f}}\right)$. Finally, estimates of the parameters are evaluated from (5.37)-(5.40) as

$$
\begin{aligned}
\hat{\boldsymbol{\mu}}^{\mathrm{r}} & =-\arg \left(\operatorname{diag}\left(\boldsymbol{\Xi}_{\mathrm{r}}\right)\right) \\
\hat{\boldsymbol{\gamma}} & =\arg \left(\operatorname{diag}\left(\boldsymbol{\Xi}_{\mathrm{d}}\right)\right) \\
\hat{\boldsymbol{\mu}}^{\mathrm{t}} & =-\arg \left(\operatorname{diag}\left(\boldsymbol{\Xi}_{\mathrm{t}}\right)\right) \\
\hat{\boldsymbol{\eta}} & =-\arg \left(\operatorname{diag}\left(\boldsymbol{\Xi}_{\mathrm{f}}\right)\right)
\end{aligned}
$$




\section{Complex Amplitude Estimation}

We assume that the complex amplitude of each path is equal for all antenna pairs, which is reasonable considering the separation of gain $\beta_{z}$ from array dependent steering vectors $\mathbf{a}_{\mathrm{r}}$ and $\mathbf{a}_{\mathrm{t}}$ in (5.6). The complex amplitudes can therefore be estimated via a least square fit to the known channel. Using the Vandermonde structure of $\mathbf{A}$ and (5.6), we form the following equation for the first entry of $\hat{\mathbf{H}}$ in (5.9) for the first subcarrier

$$
\left[\begin{array}{c}
\hat{h}_{11}(1) \\
\hat{h}_{11}(2) \\
\vdots \\
\hat{h}_{11}(Q)
\end{array}\right]=\left[\begin{array}{ccc}
1 & \cdots & 1 \\
e^{j \hat{\nu}_{1}} & \cdots & e^{j \hat{\nu}_{\hat{z}}} \\
\vdots & \ddots & \vdots \\
e^{j(Q-1) \hat{\nu}_{1}} & \cdots & e^{j(Q-1) \hat{\nu}_{\hat{Z}}}
\end{array}\right]\left[\begin{array}{c}
\beta_{1} \\
\beta_{2} \\
\vdots \\
\beta_{\hat{Z}}
\end{array}\right]+\left[\begin{array}{c}
n(1) \\
n(2) \\
\vdots \\
n(Q)
\end{array}\right]
$$

which can be written in matrix form as

$$
\hat{\mathbf{h}}_{11}=\hat{\mathbf{W}} \boldsymbol{\beta}+\mathbf{n}
$$

$\boldsymbol{\beta}$ can be obtained via a regularized LS solution of (5.46) as

$$
\hat{\boldsymbol{\beta}}=\left(\hat{\mathbf{W}}^{H} \hat{\mathbf{W}}+\sigma \mathbf{I}\right) \hat{\mathbf{W}}^{H} \hat{\mathbf{h}}_{11}
$$

where $\sigma$ is the regularization parameter chosen to minimize the effects of errors in $\hat{\mathbf{W}}$ on the estimation. For the rest of this thesis $\sigma$ is chosen empirically as $10^{-5}$. Note that although (5.47) gives an estimate of the complex amplitudes, our preliminary simulations show that improved estimates can be obtained by using more entries of $\hat{\mathbf{H}}$ in the estimation. We therefore generalize (5.46) as

$$
\hat{\mathbf{h}}_{n m}=\hat{\mathbf{W}}_{n m} \boldsymbol{\beta}+\mathbf{n} \quad \forall n \in[1, N] \quad m \in[1, M]
$$

with $\hat{\mathbf{W}}_{n m}$ defined as

$$
\hat{\mathbf{W}}_{n m}=\mathbf{v}_{n}^{r} \diamond \hat{\mathbf{W}} \diamond \mathbf{v}_{m}^{t}
$$

where $\diamond$ denotes the Khatri-Rao product, and $\mathbf{v}_{n}^{r}$ is defined as

$$
\mathbf{v}_{n}^{r}=\left[\begin{array}{llll}
e^{j(n-1) \mu_{1}^{r}} & e^{j(n-1) \mu_{2}^{r}} & \cdots & e^{j(n-1) \mu_{\hat{Z}}^{r}}
\end{array}\right]
$$


$\mathbf{v}_{m}^{t}$ is defined analogously. We combine the $N M$ equations in (5.48) and solve for $\hat{\boldsymbol{\beta}}$ as

$$
\hat{\boldsymbol{\beta}}=\left(\hat{\mathbf{W}}_{D}^{H} \hat{\mathbf{W}}_{D}+\sigma \mathbf{I}\right) \hat{\mathbf{W}}_{D}^{H} \hat{\mathbf{h}}
$$

where $\hat{\mathbf{h}}=\left[\begin{array}{llll}\hat{\mathbf{h}}_{11}^{T} & \hat{\mathbf{h}}_{12} & \cdots & \hat{\mathbf{h}}_{N M}\end{array}\right]$ and $\hat{\mathbf{W}}_{D}=\left[\begin{array}{lllll}\hat{\mathbf{W}}_{11} & \hat{\mathbf{W}}_{12} & \cdots & \hat{\mathbf{W}}_{N M}\end{array}\right]^{T}$. It should be noted that the choice of using (5.47) or (5.51) is a compromise between complexity and accuracy, since the improved amplitude estimates in (5.51) is achieved at the cost of increased computational complexity. We will utilize (5.51) for our analysis in this chapter.

\section{Channel Prediction}

Once the parameters of the model have been estimated, the time-varying frequency selective channel is predicted via

$$
\tilde{\mathbf{H}}(q, k)=\sum_{z=1}^{\hat{Z}} \hat{\beta}_{z} \mathbf{a}_{r}\left(\hat{\mu}_{z}^{\mathrm{r}}\right) \mathbf{a}_{t}^{T}\left(\hat{\mu}_{z}^{\mathrm{t}}\right) e^{j q \hat{\nu}_{z}-j k \hat{\eta}_{z}}
$$

\subsubsection{TSSM-WIMEMCHAP}

Unlike the DOD/DOA-WIMEMCHAP approach, the TSSM-WIMEMCHAP involves 3D parameter estimation. The steps involved in the TSSM-WIMEMCHAP approach are described below.

\section{Covariance Matrix Estimation}

The covariance matrix, containing the receive spatial, temporal and frequency correlations, is estimated from (5.16) as

$$
\mathbf{C}_{\mathrm{t}}=\frac{1}{M R T} \hat{\mathbf{X}}_{\mathrm{t}} \hat{\mathbf{X}}_{\mathrm{t}}^{H}
$$




\section{Number of Paths Estimation and Subspace Decomposition}

We again estimate $Z$ using the MMSE-MDL criterion in (5.28) and eigendecompose $\mathbf{C}_{\mathrm{t}}$ as

$$
\mathbf{C}_{\mathrm{t}}=\mathbf{E}_{\mathrm{s}} \Lambda_{\mathrm{s}} \mathbf{E}_{\mathrm{s}}^{H}+\mathbf{E}_{\mathrm{n}} \Lambda_{\mathrm{n}} \mathbf{E}_{\mathrm{n}}^{H}
$$

\section{Parameter Estimation}

Extraction of the parameter sets $\left\{\mu_{z}^{r}, \gamma_{z}, \eta_{z}\right\}_{z=1}^{\hat{Z}}$ requires a $3 \mathrm{D}$ estimation procedure. Similar to (4.98), we form the following 3D selection matrices

$$
\begin{aligned}
& \mathbf{J}_{\mathrm{r} 1}=\mathbf{I}_{S} \otimes \mathbf{I}_{U} \otimes \mathbf{J}_{1 \mathrm{r}} \\
& \mathbf{J}_{\mathrm{r} 2}=\mathbf{I}_{S} \otimes \mathbf{I}_{U} \otimes \mathbf{J}_{2 \mathrm{r}} \\
& \mathbf{J}_{\mathrm{d} 1}=\mathbf{I}_{S} \otimes \mathbf{J}_{1 \mathrm{~d}} \otimes \mathbf{I}_{N} \\
& \mathbf{J}_{\mathrm{d} 2}=\mathbf{I}_{S} \otimes \mathbf{J}_{2 \mathrm{~d}} \otimes \mathbf{I}_{N} \\
& \mathbf{J}_{\mathrm{f} 1}=\mathbf{J}_{1 \mathrm{f}} \otimes \mathbf{I}_{U} \otimes \mathbf{I}_{N} \\
& \mathbf{J}_{\mathrm{f} 2}=\mathbf{J}_{2 \mathrm{f}} \otimes \mathbf{I}_{U} \otimes \mathbf{I}_{N}
\end{aligned}
$$

Using the selection matrices in (5.55), we form 3D invariance equations analogous to (5.30) and solve (5.32)-(5.34), (5.35), (5.36), (5.38)-(5.40) and (5.42)-(5.44) to obtain the parameter estimates.

\section{TSS Estimation}

The TSS can be similarly obtained via a least square approach. We assume that the TSS of the scattering sources are equal for all subcarriers and use the channel for the first subcarrier in the estimation ${ }^{2}$. Let $\mathbf{s}^{m}=$ $\left[\begin{array}{llll}\mathbf{s}_{1}(m) & \mathbf{s}_{2}(m) & \cdots & \mathbf{s}_{\hat{Z}}(m)\end{array}\right]^{T}$ be a vector containing the $m$ th entry of the TSS for all paths. Using (5.7), we obtain

$$
\hat{\mathbf{h}}_{m}=\hat{\mathbf{W}}_{m} \mathbf{s}^{m}+\mathbf{n} \quad \forall m \in[1, M]
$$

\footnotetext{
${ }^{2}$ The accuracy of the TSS estimation may be improved by incorporating all of the pilot subcarriers. However, the computational complexity will scale with the number of subcarriers.
} 
where $\hat{\mathbf{h}}_{m}=\left[\hat{h}_{1 m}(1), \hat{h}_{1 m}(2), \cdots, \hat{h}_{1 m}(Q), \cdots, \hat{h}_{N m}(Q)\right]^{T}$ and $\hat{\mathbf{W}}_{m}$ is defined as

$$
\hat{\mathbf{W}}_{m}=\mathbf{A}_{\mathrm{r}} \diamond \hat{\mathbf{W}}
$$

where $\mathbf{A}_{\mathrm{r}}$ is the receive array steering matrix defined for the ULA in (4.27). We solve (5.56) using the least square approach and estimate the TSS for each path as

$$
\hat{\mathbf{s}}_{z}=\left[\begin{array}{llll}
\hat{\mathbf{s}}^{1}(z) & \hat{\mathbf{s}}^{2}(z) & \cdots & \hat{\mathbf{s}}^{M}(z)
\end{array}\right]^{T}
$$

\section{Channel Prediction}

Extrapolation of the channel using the TSSM-WIMEMCHAP is obtained from

$$
\tilde{\mathbf{H}}(q, k)=\sum_{z=1}^{\hat{Z}} \mathbf{a}_{r}\left(\hat{\mu}_{z}^{\mathrm{r}}\right) \hat{\mathbf{s}}_{z}^{T} e^{j q \hat{\nu}_{z}-j k \hat{\eta}_{z}}
$$

\subsubsection{MSSM-WIMEMCHAP}

This approach involves estimation of the Doppler shifts and delays, which can be achieved via a $2 \mathrm{D}$ estimation procedure. This method is essentially an extension of the SISO schemes in [118] to MIMO channels. A summary of the steps in the prediction is given below.

\section{Covariance Matrix Estimation}

The time-frequency covariance matrix is estimated using

$$
\mathbf{C}_{\mathrm{m}}=\frac{1}{N M R T} \hat{\mathbf{X}}_{\mathrm{m}} \hat{\mathbf{X}}_{\mathrm{m}}^{H}
$$

\section{Number of Paths Estimation and Subspace Decomposition}

We estimate the number of paths using the MMSE-MDL criterion in (5.28) with the eigenvalues of $\mathbf{C}_{\mathrm{M}}$. The eigendecomposition of $\mathbf{C}_{\mathrm{M}}$ can thus be expressed as

$$
\mathbf{C}_{\mathrm{M}}=\mathbf{E}_{\mathrm{s}} \Lambda_{\mathrm{s}} \mathbf{E}_{\mathrm{s}}^{H}+\mathbf{E}_{\mathrm{n}} \Lambda_{\mathrm{n}} \mathbf{E}_{\mathrm{n}}^{H}
$$




\section{Parameter Estimation}

Extraction of the parameter sets $\left\{\gamma_{z}, \eta_{z}\right\}_{z=1}^{\hat{Z}}$ requires $2 \mathrm{D}$ estimation and four selection matrices $\mathbf{J}_{\mathrm{d} 1}, \mathbf{J}_{\mathrm{d} 2}, \mathbf{J}_{\mathrm{f} 1}$ and $\mathbf{J}_{\mathrm{f} 2}$ defined analogously to (4.73). We solve (5.33)-(5.34), (5.35)-(5.36), (5.39)-(5.40) and (5.43)-(5.44) to obtain the parameter estimates.

\section{MSS Estimation}

Let $\mathbf{s}_{n m}=\left[\begin{array}{llll}\mathbf{S}_{1}(n, m) & \mathbf{S}_{2}(n, m) & \cdots & \mathbf{S}_{Z}(n, m)\end{array}\right]^{T} \in \mathbb{C}^{Z \times 1}$ be a vector containing the $(n, m)$ th entry of the MSS for all paths. Using (5.7), it can be easily shown that

$$
\hat{\mathbf{h}}_{n m}=\hat{\mathbf{W}}_{11} \mathbf{s}_{n m}+\mathbf{n}
$$

for $n \in[1, N]$ and $m \in[1, M]$. For all antenna pairs, we find the least square solution $\hat{\mathbf{s}}_{n m}$ to $(5.62)$ and compute the MSS for the $z$ th path as

$$
\hat{\mathbf{S}}_{z}=\left[\begin{array}{cccc}
\hat{\mathbf{s}}_{11}(z) & \hat{\mathbf{s}}_{12}(z) & \cdots & \hat{\mathbf{s}}_{1 M}(z) \\
\hat{\mathbf{s}}_{21}(z) & \hat{\mathbf{s}}_{22}(z) & \cdots & \hat{\mathbf{s}}_{2 M}(z) \\
\vdots & \vdots & \ddots & \vdots \\
\hat{\mathbf{s}}_{N 1}(z) & \hat{\mathbf{s}}_{N 2}(z) & \cdots & \hat{\mathbf{s}}_{N M}(z)
\end{array}\right]
$$

\section{Channel Prediction}

Finally, channel prediction using the MSSM-WIMEMCHAP is achieved using

$$
\tilde{\mathbf{H}}(q, k)=\sum_{z=1}^{\hat{Z}} \hat{\mathbf{S}}_{z} e^{j q \hat{\nu}_{z}-j k \hat{\eta}_{z}}
$$

\subsection{Cluster Based Prediction Method}

In Section 5.3, we presented algorithms for the prediction of pilot based wideband MIMO channels. While the proposed methods show reasonable performance for environments with few number of scatterers, the performance 
degrades with increasing number of paths. In order to effectively model the channel, recent standards use the concept of ray clustering where rays are grouped into paths based on their delays. The idea of ray clustering can also be utilized to increase the number of resolvable rays in channel parameter estimation. Consider the WINNER channel model for example, where rays within a cluster/path share a common delay [3]. By separating estimation of the common parameter (i.e., delays) from that of other parameters, the number of estimated parameters is decreased by $\left(R_{p}-1\right) P^{3}$. An alternative prediction method based on the clustering concept in recent standardized channel models is proposed in this section. We will refer to the cluster based approach which has been published in [16] as CBM-WIMEMCHAP. Given the model in (5.5) and the $K Q$ channel estimates in (5.7), as shown in Fig. 5.1, the proposed algorithm estimates the cluster delays, jointly estimates the spatial and temporal parameters of each cluster and applies the estimated parameters to predict future states of the channel.

\subsubsection{Cluster Parameter Estimation}

The cluster parameter estimation stage involves estimating the number of clusters and cluster delays using the channel frequency correlations followed by estimation of the cluster impulse responses. We form a Hankel matrix using the $K$ frequency domain pilot channel frequency responses for each of the $Q$ time symbols as

$$
\hat{\mathbf{D}}(i)=\left[\begin{array}{cccc}
\hat{\mathbf{h}}^{T}(i, 1) & \hat{\mathbf{h}}^{T}(i, 2) & \cdots & \hat{\mathbf{h}}^{T}\left(i, S_{f}\right) \\
\hat{\mathbf{h}}^{T}(i, 2) & \hat{\mathbf{h}}^{T}(i, 3) & \cdots & \hat{\mathbf{h}}^{T}\left(i, S_{f}+1\right) \\
\vdots & \vdots & \ddots & \vdots \\
\hat{\mathbf{h}}^{T}\left(i, T_{f}\right) & \hat{\mathbf{h}}^{T}\left(i, T_{f}+1\right) & \cdots & \hat{\mathbf{h}}^{T}(i, K)
\end{array}\right]
$$

\footnotetext{
${ }^{3}$ For the urban macro NLOS scenarios with a total of 400 rays grouped into 20 clusters, this corresponds to a reduction of the number of parameters to be estimated from 2400 to 2020.
} 


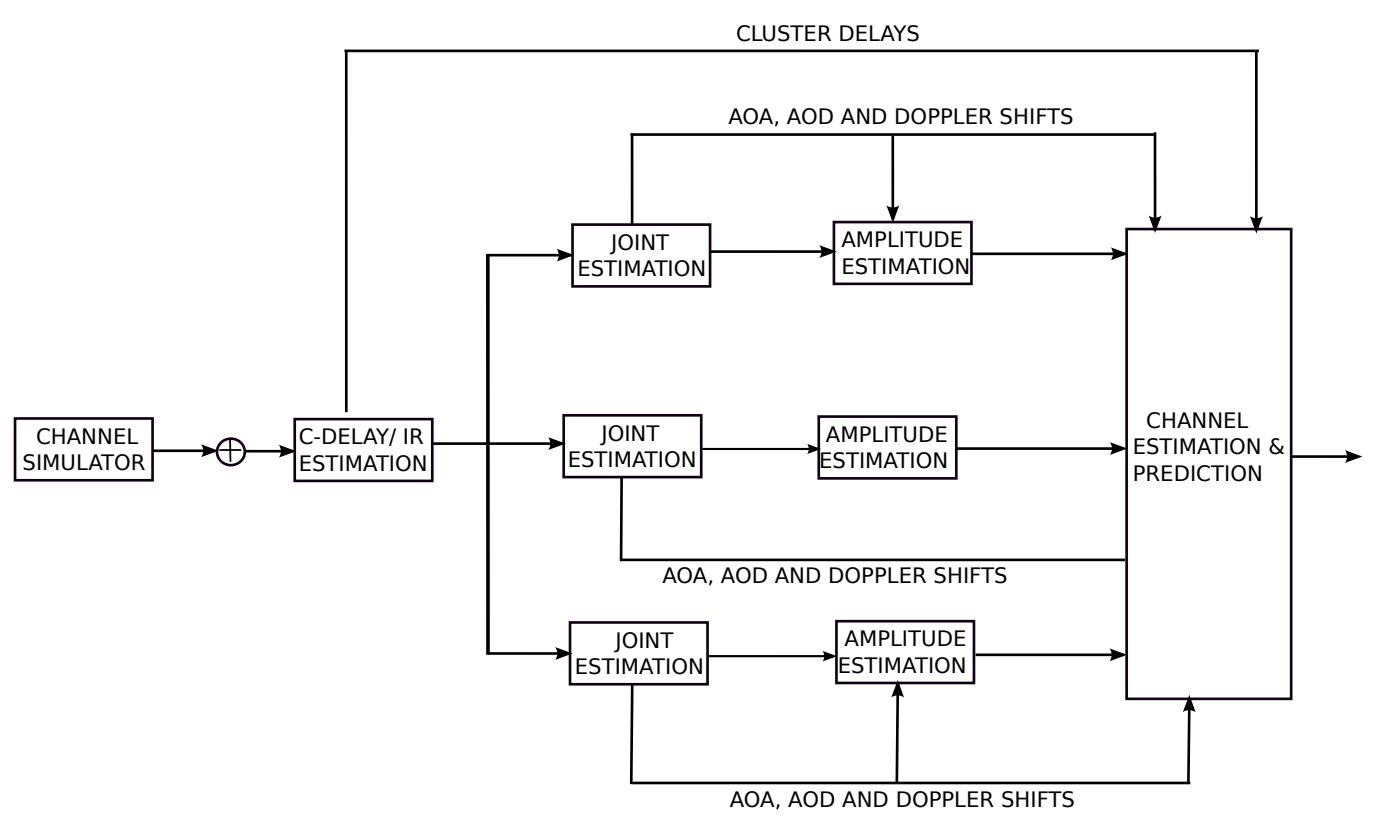

Figure 5.1: Block diagram of the proposed cluster based channel prediction scheme.

where $\hat{\mathbf{h}}(q, k)=\operatorname{vec}[\hat{\mathbf{H}}(q, k)]$ is the vectorised form of the channel response obtained by stacking its columns. $S_{f}$ and $T_{f}=K-S_{f}+1$ are the Hankel matrix size parameters which determine the size of the covariance matrix and the number of correlation averages. $T_{f}$ is chosen such than $C_{\max }<T_{f}<K^{4}$. The frequency correlation matrix averaged over the $N_{t}$ time domain pilots is obtained as

$$
\hat{\mathbf{C}}_{f}=\frac{1}{N M Q S_{f}} \sum_{i=1}^{Q} \hat{\mathbf{D}}(i) \hat{\mathbf{D}}(i)
$$

\footnotetext{
${ }^{4}$ We assume that the maximum number of clusters $C_{\max }$ is known apriori. This is reasonable since $C_{\max }$ is determined by the propagation environment.
} 


\section{Number of Clusters Estimation}

As in the previous methods, we propose to utilize the MMDL [85] for estimating the number of clusters,

$$
\hat{C}=\arg \min _{1 \leq u \leq N_{f}-1} N M Q S_{f} \log \left(\lambda_{u}\right)+\frac{1}{2}\left(u^{2}+u\right) \log \left(N M Q S_{f}\right)
$$

where $\lambda_{u}, u=1, \cdots, N_{f}$, are the eigenvalues of $\hat{\mathbf{C}}_{f}$.

\section{Cluster Delay Estimation}

Similar to the SISO estimation and prediction algorithms in [205,214], we propose an ESPRIT [158] based approach for the cluster delay estimation stage of the prediction scheme. Letting $\mathbf{E}_{\mathrm{s}}$ be the signal subspace matrix containing the eigenvectors corresponding to the $\hat{C}$ largest eigenvalues of $\hat{\mathbf{C}}_{f}$, we form two subarray matrices with maximum overlap as

$$
\begin{aligned}
& \mathbf{E}_{\mathrm{s} 1}=\left[\begin{array}{ll}
\mathbf{I}_{N_{f}-1} & \mathbf{0}
\end{array}\right] \mathbf{E}_{\mathrm{s}} \\
& \mathbf{E}_{\mathrm{s} 2}=\left[\begin{array}{ll}
\mathbf{0} & \mathbf{I}_{N_{f}-1}
\end{array}\right] \mathbf{E}_{\mathrm{s}}
\end{aligned}
$$

where $\mathbf{I}_{N_{f}-1} \in \mathbb{R}^{\left(N_{f}-1\right) \times\left(N_{f}-1\right)}$ is the identity matrix and $\mathbf{0}$ is a $\left(N_{f}-1\right) \times 1$ vector of zeros. We form the $1 \mathrm{D}$ invariance equation as

$$
\mathrm{E}_{\mathrm{s} 1} \Phi=\mathrm{E}_{\mathrm{s} 1}
$$

where $\boldsymbol{\Phi} \in \mathbb{C}^{N_{f} \times N_{f}}$ is a subspace rotation matrix whose eigenvalues give the normalized delay estimates. Equation (5.69) can then be solved in the least square sense to obtain

$$
\boldsymbol{\Phi}=\left(\mathbf{E}_{\mathrm{s} 1}^{H} \mathbf{E}_{\mathrm{s} 1}\right)^{-1} \mathbf{E}_{\mathrm{s} 1}^{H} \mathbf{E}_{\mathrm{s} 2}
$$

If $\left\{\mu_{c}\right\}_{c=1}^{\hat{C}}$ are the $\hat{C}$ eigenvalues of $\boldsymbol{\Phi}$, the delay of the $c$ th cluster is estimated as

$$
\hat{\tau}_{c}=\frac{\arg \left[\mu_{c}\right]}{2 \pi \Delta f}
$$




\section{Scattering Co-efficient Estimation}

Once the cluster delays have been estimated, an estimate of the $\hat{C} \times Q$ matrix $\mathcal{H}_{n m}$ containing the scattering coefficient for the channel between the $m$ th receive and $n$th transmit antenna is obtained using the least square solution as

$$
\hat{\mathcal{H}}_{n m}=\left(\mathbf{F}^{H} \mathbf{F}+\eta \mathbf{I}\right)^{-1} \mathbf{F} \hat{\mathbf{H}}_{n m}
$$

where $\eta$ is the regularizing parameter introduced to minimize the effects of cluster delay estimation error on the solution of (5.72). F is the $K \times \hat{C}$ Fourier transform matrix with $[\mathbf{F}]_{a, b}=\exp \left(-j 2 \pi a \Delta f \tau_{b}\right)$ and $\hat{\mathbf{H}}_{n m}$ is a $K \times Q$ matrix containing the estimated frequency domain pilot channel between the $m$ th receive and $n$th transmit antenna elements over the $Q$ pilot symbol periods. Estimates of the MIMO channel response for the $c$ th cluster at the $q$ th time instant is obtained from the solutions of (5.72) as

$$
\hat{\mathbf{H}}_{c}(q)=\left[\begin{array}{cccc}
\hat{\mathcal{H}}_{11}(c, q) & \hat{\mathcal{H}}_{12}(c, q) & \ldots & \hat{\mathcal{H}}_{1 M}(c, q) \\
\hat{\mathcal{H}}_{21}(c, q) & \hat{\mathcal{H}}_{22}(c, q) & \ldots & \hat{\mathcal{H}}_{2 M}(c, q) \\
\vdots & \vdots & \ddots & \vdots \\
\hat{\mathcal{H}}_{N 1}(c, q) & \hat{\mathcal{H}}_{N 2}(c, q) & \cdots & \hat{\mathcal{H}}_{N M}(c, q)
\end{array}\right]
$$

\subsubsection{Joint Angle and Doppler Estimation}

Given the estimates in (5.73) and the model in (5.59), the AOA, AOD and Doppler shifts can be jointly extracted for the rays within each of the $\hat{C}$ clusters. Although this stage is similar to the parameter estimation stage of the DOD/DOA-MEMCHAP in Section 4.4.4, we briefly summarize the estimation procedure for clarity. Using the $Q$ estimates in (5.73), we estimate the spatio-temporal correlation matrix for the $c$ th cluster as

$$
\hat{\mathbf{C}}_{t}^{c}=\frac{1}{S_{t}}\left(\hat{\mathbf{D}}_{c} \hat{\mathbf{D}}_{c}^{H}\right)
$$


where $\hat{\mathbf{D}}_{c}$ is a $N M T_{t} \times S_{t}$ Hankel matrix defined as

$$
\hat{\mathbf{D}}_{c}=\left[\begin{array}{cccc}
\hat{\mathbf{h}}_{c}(1) & \hat{\mathbf{h}}_{c}(2) & \ldots & \hat{\mathbf{h}}_{c}\left(S_{t}\right) \\
\hat{\mathbf{h}}_{c}(2) & \hat{\mathbf{h}}_{c}(3) & \cdots & \hat{\mathbf{h}}_{c}\left(S_{t}+1\right) \\
\vdots & \vdots & \ddots & \vdots \\
\hat{\mathbf{h}}_{c}\left(T_{t}\right) & \hat{\mathbf{h}}_{c}\left(T_{t}+1\right) & \cdots & \hat{\mathbf{h}}_{c}\left(N_{t}\right)
\end{array}\right]
$$

and $\hat{\mathbf{h}}_{c}(q)$ is the $N M \times 1$ vector obtained by stacking the columns of $\hat{\mathbf{H}}_{c}(q)$, $T_{t}=Q-S_{t}+1$.

\section{Number of Rays Estimation}

The number of rays $R_{c}$ is estimated as $\hat{R}_{c}$ using the MMSE-MDL given in (5.68) with the eigenvalues of $\hat{\mathbf{C}}_{f}$ replaced with the eigenvalues of $\hat{\mathbf{C}}_{t}^{c}$ and $K$ with $Q$.

\section{ESPRIT Based Angle and Doppler Estimation}

Let $\mathbf{E}_{s}$ be the signal subspace matrix containing the eigenvectors corresponding to the $\hat{R}_{c}$ largest eigenvalues of $\hat{C}_{t}^{c}$. The parameters $\left\{\mu_{r, p}^{\mathrm{r}}, \mu_{r, p}^{\mathrm{t}}, \nu_{r, p}\right\}_{r=1}^{R_{p}} \forall p=$ $1, \cdots, \hat{P}$ are then estimated from $\mathbf{E}_{s}$ via a $3 \mathrm{D}$ ESPRIT procedure analogous to that described in Section 4.4.4.

\section{Complex Amplitude Estimation}

Once the parameters of the rays within each of the clusters have been estimated, estimation of the complex amplitudes $\beta_{r, p}$ of (5.5) can be achieved by minimizing the MSE. Let $\hat{\mathbf{h}}_{11}^{p}$ be the $1 \times Q$ vector obtained from the $p$ th row of $\mathcal{H}_{11}$. The complex amplitudes of the rays in the $p$ th cluster are obtained from $^{5}$

$$
\hat{\boldsymbol{\beta}}_{p}=\left(\mathbf{G}_{p}^{H} \mathbf{G}_{p}+\eta \mathbf{I}\right)^{-1} \mathbf{G}_{p} \hat{\mathbf{h}}_{11}^{p}
$$

\footnotetext{
${ }^{5}$ To minimize algorithm complexity, we consider the single entry amplitude estimation.
} 
Table 5.3: Propagation Channel Parameters (Scenario I)

\begin{tabular}{llllll}
\hline Path & \multicolumn{5}{c}{ Parameters } \\
\cline { 2 - 6 } & $\beta_{z}$ & $\theta_{z}$ & $\phi_{z}$ & $\tau_{z}(\mathrm{~ns})$ & $\nu_{z}(\mathrm{rad} / \mathrm{s})$ \\
\hline 1 & $-0.76+0.074 \mathrm{j}$ & 0.49 & -2.90 & 0 & 185.10 \\
2 & $-0.76+0.30 \mathrm{j}$ & -1.89 & 0.99 & 60 & -462.10 \\
3 & $-1.41+0.14 \mathrm{j}$ & -2.48 & 2.99 & 75 & 497.31 \\
4 & $0.16-1.15 \mathrm{j}$ & -1.88 & 1.46 & 145 & -331.90 \\
5 & $0.37-0.82 \mathrm{j}$ & -2.66 & 2.05 & 150 & 208.61 \\
6 & $-0.33+1.04 \mathrm{j}$ & -0.02 & -1.60 & 155 & -156.92 \\
\hline
\end{tabular}

where $\hat{\boldsymbol{\beta}}_{p}=\left[\hat{\beta}_{1, p}, \cdots, \hat{\beta}_{\hat{R}_{p}, p}\right]^{T}$ and the $Q \times \hat{R}_{p}$ Vandermonde structured matrix $\hat{\mathbf{G}}_{p}$ is defined as

$$
\hat{\mathbf{G}}_{1}=\left[\begin{array}{ccc}
1 & \cdots & 1 \\
e^{j \nu_{1, p}} & \cdots & e^{j \nu_{\hat{R}_{p}, p}} \\
\vdots & \ddots & \vdots \\
e^{j(Q-1) \nu_{1, p}} & \cdots & e^{j(Q-1) \nu_{\hat{R}_{p}, p}}
\end{array}\right]
$$

\subsubsection{Channel Prediction}

Having estimated the parameters of the doubly selective channel, prediction of the channel is achieved by substituting the parameters into the model for the desired frequency and temporal instants. The predicted CSI is thus

$$
\mathbf{H}(q, k)=\sum_{p=1}^{\hat{P}} \sum_{r=1}^{\hat{R}_{p}} \beta_{r, p} \mathbf{a}_{\mathrm{r}}\left(\hat{\mu}_{r, p}^{\mathrm{r}}\right) \mathbf{a}_{\mathrm{t}}^{T}\left(\hat{\mu}_{r, p}^{\mathrm{t}}\right) e^{j\left(q \hat{\nu}_{r, p}-k \hat{\eta}_{p}\right)}
$$




\subsection{Performance Evaluation}

In this section, we evaluate the performance of the proposed schemes. After describing the performance metrics and simulation parameters, we evaluate the parameter estimation accuracy, followed by the overall prediction performance.

\subsubsection{Performance Metrics}

The overall performance of the prediction schemes is evaluated using Monte Carlo simulations with synthetic data and compared with prediction error bounds in Section 8.1.2. The normalized mean square error (NMSE) is used as the performance metric. We first define the normalized square error (NSE) over a single realization of the channel as

$$
\operatorname{NSE}(q, k)=\frac{\|\tilde{\mathbf{H}}(q, k)-\mathbf{H}(q, k)\|_{F}^{2}}{\mathbb{E}\left[\|\mathbf{H}(q, k)\|_{F}^{2}\right]}
$$

The expectation in (5.79) is approximated over the available temporal and frequency samples.

The performance of the parameter estimation stage is evaluated in terms of the root mean square error (RMSE) defined, for a generic variable $x$, as

$$
\operatorname{RMSE}(\hat{x})=\sqrt{\frac{1}{N_{\mathrm{c}}} \sum_{c=1}^{N_{\mathrm{c}}}\left(x-\hat{x}_{c}\right)^{2}}
$$

where $N_{\mathrm{c}}$ denotes the number of channel and/or noise realizations and $\hat{x}_{c}$ is the estimate of $x$ during the $c$ th realization. The NMSE and RMSE are obtained by averaging (5.79) and (5.80) over 500 realizations of the channel and/or noise. The RMSE is compared with the square root of the CRB (i.e., diagonal entries of the inverse of (8.40)). 


\subsubsection{Simulation Parameters}

We consider a MIMO-OFDM system with bandwidth $B=20 \mathrm{MHz}, N_{\mathrm{sc}}=$ 1024 subcarriers including $K=64$ equally spaced pilot subcarriers. The transmit and receive antenna arrays are both 2-element arrays with interantenna spacing $d_{\mathrm{r}}=d_{\mathrm{t}}=\lambda / 2$. We consider a carrier frequency of $f_{\mathrm{c}}=$ $2.1 \mathrm{GHz}$ and mobile velocity of $v=50 \mathrm{~km} / \mathrm{h}$. Except where otherwise stated, we use 50 samples at a sampling rate of $10 / \lambda$. In order to investigate the effects of noise and/or parameter distribution on the algorithm performance, we consider two methods of generating the channel parameters $\beta_{z}, \theta_{z}, \phi_{z}, \nu_{z}$. In simulation Scenario I, these are fixed to values given in Table 5.3 for all realizations of $\mathbf{H}$ in (5.6). In simulation Scenario II, they are randomly generated for each channel realization. The amplitudes are generated as complex Gaussian distributed random variables, $\beta_{z} \sim \mathcal{C N}(0,1)$. The angles of arrival and departure are assumed to be uniformly distributed, i.e., $\theta_{z}, \phi_{z} \sim$ $\mathcal{U}[-\pi, \pi)$. In both cases, the path delays are selected from the Urban macro (UMA) scenario in the WINNER II/3GPP channel [106], given in Table 5.3. Unless otherwise stated, we consider a 6 -path channel with parameters in Table 5.3 and the error is averaged over 500 noise realizations. .

\subsubsection{Parameter Estimation Performance}

Since Doppler frequency and delay estimation are part of all the methods, we present results showing the accuracy of their estimates in each algorithm. Figure 5.2 presents the RMSE of Doppler estimates versus SNR with 50 and 100 known samples of the channel. We observe that the performances of the three methods improves with increasing number of samples and approaches the bound as the SNR increases. Also, we note that the DOD/DOA method outperforms the TSSM and MSSM methods at all SNR values and that the MSSM method yields the highest RMSE. A possible reason for this is the additional channel structure revealed by sampling in a higher number of dimensions. Similar observations are made in Fig. 5.3, where we plot the 
RMSE of delay estimates versus SNR.

\subsubsection{Prediction Performance}

We now evaluate the overall prediction performance of the proposed methods. Figures 5.4-5.6 correspond to simulation Scenario I. Figure 5.4 presents the NMSE versus prediction horizon (in wavelengths) at an SNR of $15 \mathrm{~dB}$. The negative values of the prediction horizon correspond to the estimation stage. Again, we observe that the DOD/DOA outperforms TSSM and MSSM methods. In Fig. 5.5, we plot the corresponding cumulative distribution function $(\mathrm{CDF})$ of the normalized square error (NSE) at a prediction interval of $1 \lambda$. The DOD/DOA method has the lowest NSE for all realizations followed by the TSSM. We also observe that utilizing the spatial information in parameter estimation and prediction in the DOD/DOA and TSSM results in a decrease of about $12 \mathrm{~dB}$ relative to MSSM.

The effects of increasing SNR on the performance of the proposed schemes is shown in Fig. 5.6 where we plot the NMSE versus SNR for a prediction horizon of $1 \lambda$. As expected, as a consequence of improved parameter estimation, the performance of the algorithms improves with increasing SNR. We observe that the performance of the DOD/DOA and TSSM methods approaches the bound as SNR increases with the DOD/DOA having the lowest NMSE over the entire SNR range considered. This agrees with observations in [110] where it was shown that the prediction error bound obtained from the DOD/DOA model is lower than that for the vector spatial signature model.

We now present results for simulation Scenario II. In Fig. 5.7, we present the NMSE versus prediction horizon at an SNR of $15 \mathrm{~dB}$. Here, we observe that the averaged performance of all methods degrades when compared to Scenario I. However, the performance of the DOD/DOA and TSSM schemes are still reasonable with a maximum NMSE of about $-12 \mathrm{~dB}$ for the TSSM and $-22 \mathrm{~dB}$ for the DOD/DOA over the $15 \lambda$ prediction horizon shown. We observe 
that the MSSM performs poorly with an NMSE of approximately $4.8 \mathrm{~dB}$ over the entire region considered. A possible explanation for the increase in NMSE is that unlike in simulation Scenario I, certain channel realizations have parameters which are closer than the resolution limit of the parameter estimation stage, leading to reduced parameter estimation and prediction performance. The CDF of the NSE corresponding to the mean results in Fig. 5.7 at a prediction interval of $1 \lambda$ is shown in Fig. 5.8.

Finally, the CDF of the prediction error of the DOD/DOA for different number of propagation paths is presented in Fig. 5.9. We observe that the performance of the algorithm degrades with increasing number of paths.

\subsubsection{Prediction Performance with WINNER II Model}

In this section, we evaluate the performance of the cluster based prediction compared to the DOD/DOA-WIMEMCHAP using the two scenarios of the WINNER II channel model [106] described in Section 2.4.3 with the simulation parameters in Section 5.5.2. Except where stated otherwise, we consider a MIMO-OFDM system with parameters given in Section 2.4.3. Figure 5.10 shows the prediction NMSE versus prediction horizon (in wavelengths) at $\mathrm{SNR}=10 \mathrm{~dB}$. We observe that the cluster based approach outperforms the non-cluster based method for the C2 (NLOS urban macro-cell) and B2 (NLOS urban micro-cell) scenarios. A plausible explanation for the performance difference is that, while the number of resolvable rays in the non-cluster based approach is much less than the total number of rays (i.e., 400 in $\mathrm{C} 2$ and 300 in B2) in the channel, estimation of the parameters of the clusters separately in the cluster based scheme results in increased number of resolvable paths. As a consequence of the higher number of clusters in the C2 channel, the algorithm produces a higher NMSE than that for the B2 channel. Similar observations are made in Fig. 5.11, where we plot the corresponding CDF of NSE at a prediction horizon of $2 \lambda$. 


\subsection{Summary}

In this chapter, we have presented different parametric schemes for the prediction of mobile MIMO-OFDM channels. The predictors are based on different formulations of the double directional model and original adaptation of multidimensional ESPRIT to jointly extract the channel parameters. Numerical simulations indicate that the performance of the algorithms approaches the error bound with increasing SNR and/or number of samples. We have quantified the parameter estimation and channel prediction improvement afforded by the spatial structure of the channel revealed by multiple sampling of the wavefield. The method utilizing both transmit and receive spatial information (DOD/DOA-WIMEMCHAP) outperform those with only receive spatial information (TSSM-WIMEMCHAP), and no spatial information (MSSMWIMEMCHAP).

An alternative approach utilizing the concept of clustering in recent standardized channel models is also proposed. The scheme estimates the cluster delay and scattering coefficients via a 1D ESPRIT approach and utilizes a 3D ESPRIT based scheme to jointly estimate the angles of arrival, angles of departure and Doppler shifts. The estimated parameters are then used to extrapolate the channel using the model. Simulation results using the industry standard 3GPP/WINNER II spatial channel model show that the cluster based algorithm offers improved prediction performance over the DOD/DOA non-cluster based approach. 


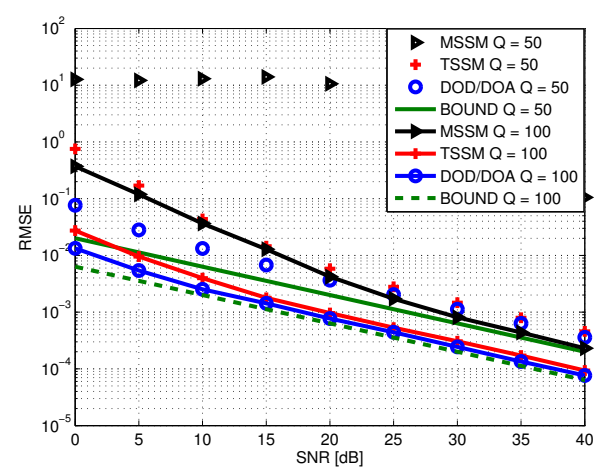

Figure 5.2: RMSE of Doppler frequency estimates versus SNR.

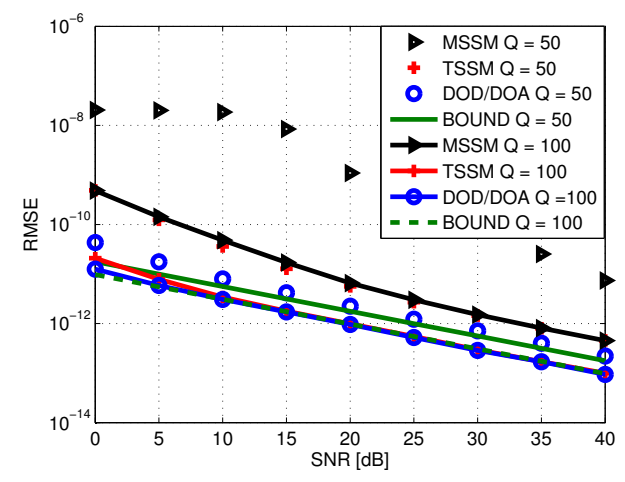

Figure 5.3: RMSE of delay estimates versus SNR.

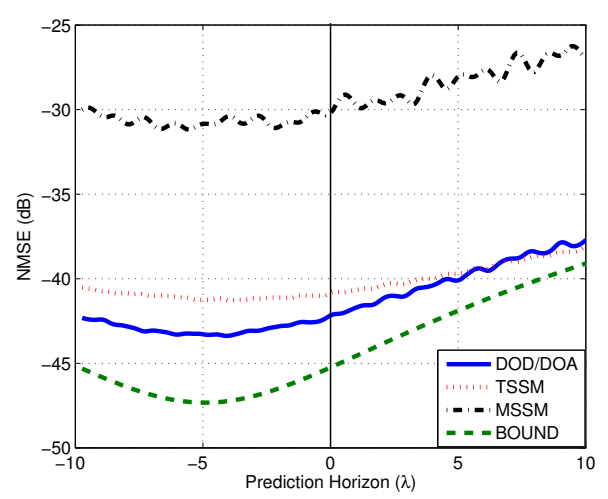

Figure 5.4: NMSE versus prediction horizon at $\mathrm{SNR}=15 \mathrm{~dB}$.

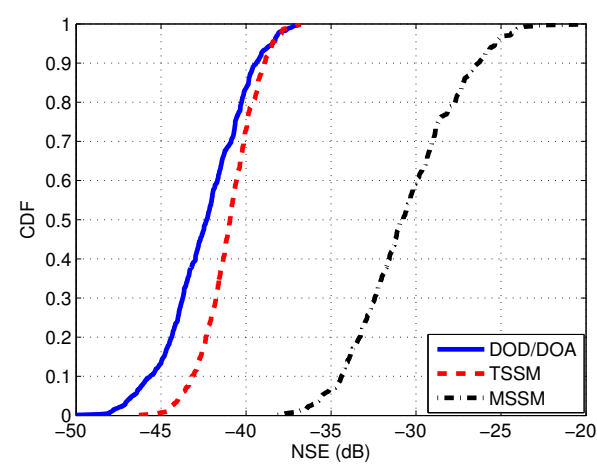

Figure 5.5: The CDF of NSE for a horizon of $1 \lambda$ at $\mathrm{SNR}=15 \mathrm{~dB}$.

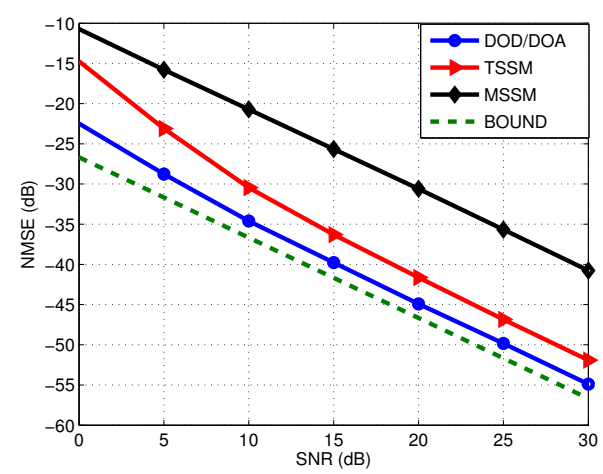

Figure 5.6: The averaged NMSE versus SNR for a horizon of $1 \lambda$.

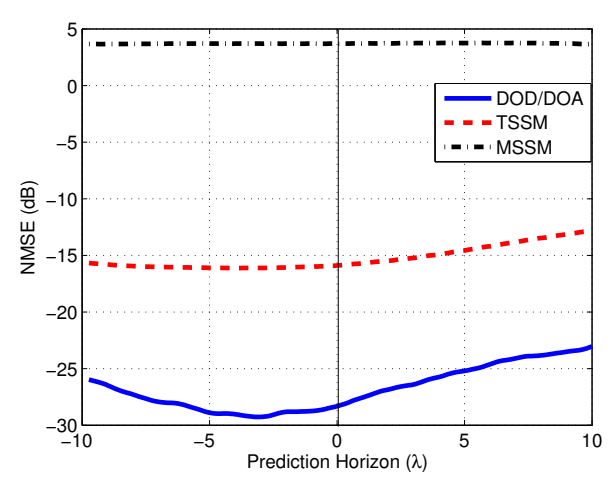

Figure 5.7: The NMSE versus prediction horizon at $\mathrm{SNR}=15 \mathrm{~dB}$. 


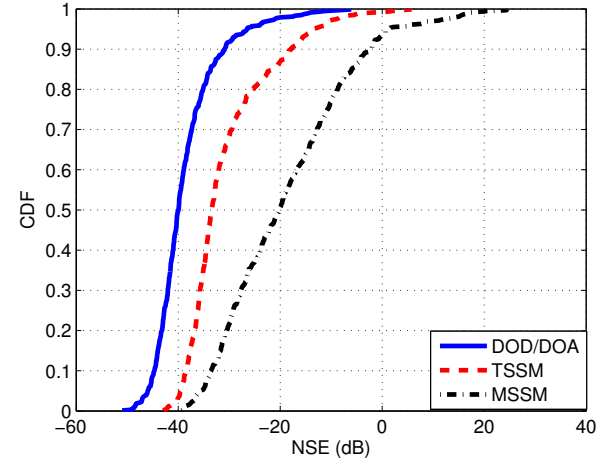

Figure 5.8: The CDF of NSE for a of $1 \lambda$ at $\mathrm{SNR}=15 \mathrm{~dB}$.

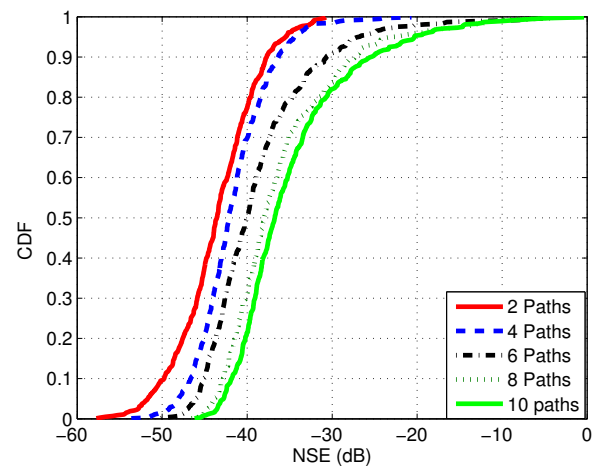

Figure 5.9: The CDF of NSE for a Figure 5.11: CDF of NSE at a preprediction of $1 \lambda$ at $\mathrm{SNR}=15 \mathrm{~dB}$.

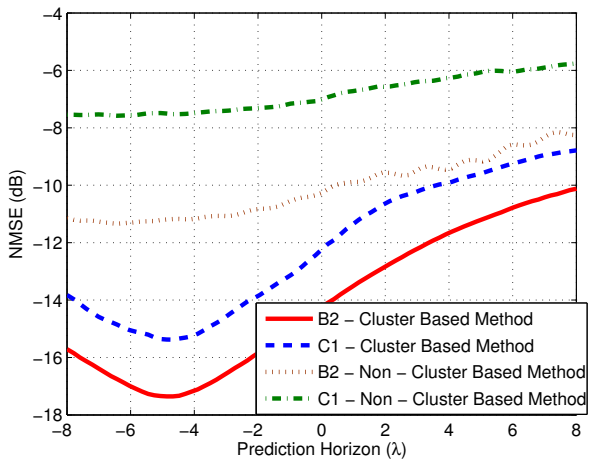

Figure 5.10: NMSE versus prediction length at $\mathrm{SNR}=10 \mathrm{~dB}$.

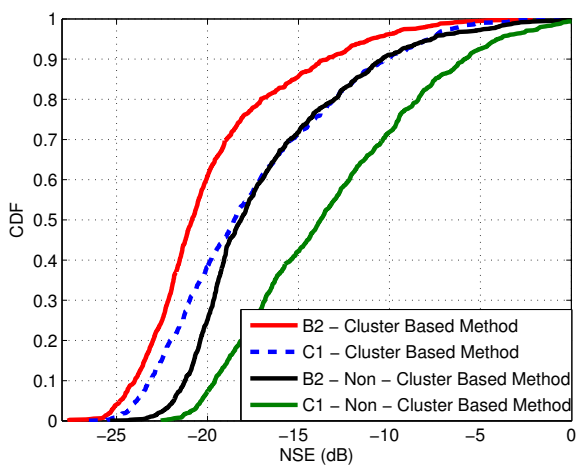

diction horizon of $2 \lambda$. 


\section{6 Mobile to Mobile Channel Prediction}

\subsection{Introduction}

/ OBILE-to-mobile (M-to-M) land wireless communication channels arise 1 when both the transmitter and receiver are moving and are equipped with low elevation antenna elements. For instance, a moving vehicle in a given location might communicate with one or more mobile vehicles in other locations. These systems have potential applications in traffic safety, rescue squad communication, congestion avoidance, etc. An international wireless standard, IEEE 802.11p, also referred to as Wireless Access in Vehicular Environments (WAVE) [4] has been developed. Based on WiFi technology, this standard is proposed for both mobile-to-mobile and mobile-to-infrastructure traffic applications.

In order to cope with the challenge of developing and evaluating the performance of current and future M-to-M wireless communication systems, 
several research results have been published on the modeling of single-input single-output (SISO) M-to-M channels. In [21,22], the statistical properties of such channels were investigated based on models for the channel impulse response and the transfer function. The authors of [194] present results on the temporal correlation properties and Doppler power spectral characteristics in 3D M-to-M propagation environments. In [127, 129], a modeling technique based on ray-tracing was used to model the vehicle-to-vehicle propagation channels. The proposed channel impulse responses capture the channel statistics in terms of time series and can therefore be used directly for link and system level simulations. Results based on measurement for outdoor-to-outdoor, outdoor-to-indoor and wideband M-to-M wireless channels have also been presented in $[9,104,128]$. Simulation models for SISO M-to-M channel were presented in [48]. These results have shown that, as a result of the increased mobility, the fading statistics of M-to-M channels differ significantly from classical fixed-to-mobile (F-to-M) channels.

Recently, modeling of M-to-M channels with multiple antenna elements at both ends of the link has received considerable research attention. In $[81,146]$, the statistical model for SISO M-to-M channel was extended to MIMO M-to-M channels. The authors modeled the scattering environment around the mobile transmitter and receiver using the classical two-ring geometrical channel model. Simulation models were developed for MIMO Mto-M channels in [219]. In [220,221], the 2D M-to-M models in [219] were extended to the modeling of 3D propagation scenarios. The authors of [47] proposed techniques for the computation of channel parameters for MIMO transmission channels with dual mobility. The schemes developed were based on a Rayleigh fading channel envelope with non-isotropic scattering. Comprehensive reviews of existing channel models, measurement campaigns and other aspects of MIMO M-to-M channel modeling can be found in [134].

Although multipath parameter estimation and channel state prediction has been thoroughly addressed for F-to-M MIMO channels (see e.g [10, 17, $143,189,193])$, there exist very few publications (to the best of the authors' 
knowledge) applicable to M-to-M MIMO systems. In [222], a maximum likelihood approach for MIMO M-to-M channel parameter estimation was developed. The computational burden and dependence on the channel model, however, makes the scheme unsuitable for practical applications. In [217, 218], schemes for the estimation of mobile velocities and direction of motion were proposed for SISO M-to-M channels. The proposed scheme is however limited to the two-ring model and extension to MIMO channels is still an open problem.

In this chapter, we extend our prediction concept of Chapter 4 to M-to-M channels. The content of this chapter has been published in part [15].

\subsection{Channel Models}

This section presents the Rayleigh fading narrowband MIMO M-to-M channel model considered in this paper along with a parametrized model for M-to-M parameter estimation and prediction.

\subsubsection{MIMO Mobile-to-Mobile Channel Model}

We consider a MIMO M-to-M wireless system with $M$ transmit and $N$ receive antenna elements. Fig. 6.1 shows an illustration of M-to-M propagation in a typical urban and suburban environment. The transmitter and receiver are assumed to be moving with velocities $v_{\mathrm{t}}$ and $v_{\mathrm{r}}$, respectively. It is further assumed that both are equipped with low elevation omnidirectional antennas. As shown in Fig. 6.1, a signal will arrive at the receiver after undergoing scattering and reflection in all directions by local objects near the transmitter and receiver as well as distant scattering objects. We assume a NLOS propagation environment, and so the complex Rayleigh faded channel for a SISO link is modeled as [21,22]

$$
h(t)=\sum_{p=1}^{P} \alpha_{p} e^{j\left[\left(\omega_{p}^{\mathrm{t}}+\omega_{p}^{\mathrm{r}}\right) t+\epsilon_{p}\right]}
$$




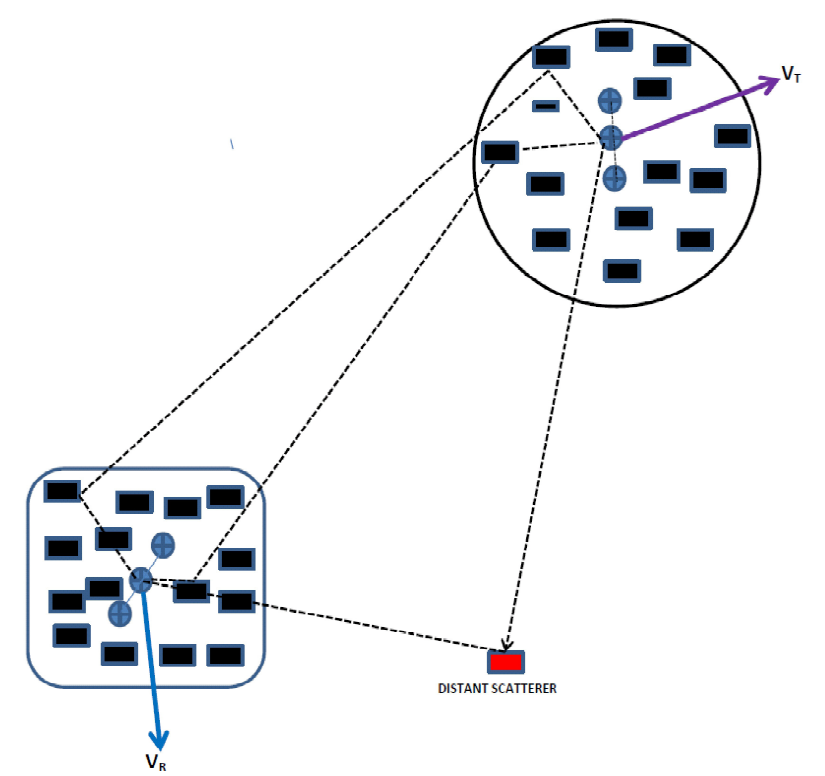

Figure 6.1: A $3 \times 3$ MIMO Mobile to Mobile wireless propagation channel characterized by local scatterers and distant scattering sources.

where $\alpha_{p}$ is the amplitude for the $p$ th path, $\epsilon_{p}$ is the $p$ th path phase parameter assumed to be uniformly distributed on $[0,2 \pi)$ and $P$ is the number of propagation paths. In (6.1), $\omega_{p}^{\mathrm{t}}$ and $\omega_{p}^{\mathrm{r}}$ are the radian Doppler shifts resulting from the mobility of the transmitter and receiver, respectively, and are given by

$$
\begin{array}{r}
\omega_{p}^{\mathrm{t}}=\frac{2 \pi}{\lambda} v_{\mathrm{t}} \sin \left(\vartheta_{p}^{\mathrm{t}}\right) \\
\omega_{p}^{\mathrm{r}}=\frac{2 \pi}{\lambda} v_{\mathrm{t}} \sin \left(\vartheta_{p}^{\mathrm{r}}\right)
\end{array}
$$

where $\vartheta_{p}^{\mathrm{t}}$ is the angle between the direction of departure of the $p$ th path and the transmitter direction of motion. $\vartheta_{p}^{\mathrm{r}}$ is the corresponding angle at the receive array and $\lambda$ is the carrier wavelength. As can be seen from (6.1), the received signal will experience Doppler frequency shifts due to the mobility of both the transmitter and receiver. The dual mobility in M-to-M channels result in more rapid temporal variation of the fading envelope when compared to classical mobile cellular systems with fixed transmitters. It should be noted 
that the sum of sinusoids model commonly used for SISO prediction studies (see e.g $[45,57,60,190]$ ) is a special case of $(6.1)$ with $v_{\mathrm{t}}=0$. The model in (6.1) can be extended to M-to-M communication channels with multiple antennas at both ends of the link by introducing the receive and transmit spatial structure, giving

$$
\mathbf{H}(t)=\sum_{p=1}^{P} \alpha_{p} \mathbf{a}_{\mathrm{r}}\left(\theta_{p}\right) \mathbf{a}_{\mathrm{t}}^{T}\left(\phi_{p}\right) e^{j\left[\left(\omega_{p}^{\mathrm{t}}+\omega_{p}^{\mathrm{r}}\right) t+\epsilon_{p}\right]}
$$

where $\mathbf{a}_{\mathrm{r}}\left(\theta_{p}\right)$ and $\mathbf{a}_{\mathrm{t}}\left(\phi_{p}\right)$ are the array geometry dependent receive and transmit steering vectors, respectively, while $\theta_{p}$ and $\phi_{p}$ are the angles of arrival and departure of the $p$ th path relative to the array broadside, respectively. For a ULA, the receive array steering vector is as given in (4.6).

\subsubsection{Parametrized Model}

We now reduce the MIMO M-to-M channel prediction problem to a multidimensional sinusoidal parameter estimation problem. In doing so, we define

$$
\beta_{p}=\alpha_{p} e^{j \epsilon_{p}}
$$

and

$$
\begin{aligned}
\omega_{p} & =\omega_{p}^{\mathrm{t}}+\omega_{p}^{\mathrm{r}} \\
& =\frac{2 \pi}{\lambda}\left(v_{\mathrm{t}} \sin \left(\vartheta_{p}^{\mathrm{t}}\right)+v_{\mathrm{r}} \sin \left(\vartheta_{p}^{\mathrm{r}}\right)\right)
\end{aligned}
$$

We will henceforth refer to $\beta_{p}$ as the complex amplitude of the $p$ th path and $\omega_{p}$ as the effective radian Doppler frequency. Substituting (6.5) and (6.6) into (6.4), we obtain

$$
\mathbf{H}(t)=\sum_{p=1}^{P} \beta_{p} \mathbf{a}_{\mathbf{r}}\left(\theta_{p}\right) \mathbf{a}_{\mathrm{t}}^{T}\left(\phi_{p}\right) e^{j \omega_{p} t}
$$

As in the previous chapters, the parameters $\beta_{p}, \theta_{p}, \phi_{p}$ and $\omega_{p}$ are assumed constant over the region of interest Assuming that the CSI is sampled at 
$T_{\text {samp }}$, by applying a parametrization similar to that in (4.8), (6.7) can be expressed as

$$
\mathbf{H}(k)=\sum_{p=1}^{P} \beta_{p} \mathbf{a}_{\mathrm{r}}\left(\mu_{p}^{\mathrm{r}}\right) \mathbf{a}_{\mathrm{t}}^{T}\left(\mu_{p}^{\mathrm{t}}\right) e^{j k \nu_{p}}
$$

where $\mu_{p}^{\mathrm{r}}$ and $\mu_{p}^{\mathrm{t}}$ are spatial frequencies which are related to the AOA and AOD, respectively and $\nu_{p}=\omega_{p} T_{\text {samp }}$ is the normalized Doppler frequency. We assume that $K$ estimates

$$
\hat{\mathbf{H}}(k)=\mathbf{H}(k)+\mathbf{N}(k)
$$

are available for estimating the parameters of the channel.

\subsection{Parameter Estimation and CSI Prediction}

As shown in (6.8), prediction of the M-to-M channel require extraction of the structural parameters $\left\{\mu_{p}^{\mathrm{r}}, \mu_{p}^{\mathrm{t}}, \nu_{p}\right\}_{p=1}^{P}$ and complex amplitudes $\left\{\beta_{p}\right\}_{p=1}^{P}$ which can be achieved using the DOD/DOA-MEMCHAP described in Section 4.4.4.

\subsubsection{Mobile Velocities Estimation}

A simple scheme for the extraction of the mobile velocities from the estimated multipath parameters is presented in this subsection. Given the $\hat{P}$ estimates of normalized parameters $\hat{\nu}_{p}, \hat{\mu}_{p}^{\mathrm{r}}$ and $\hat{\mu}_{p}^{\mathrm{t}}$, we estimate the channel parameters thus

$$
\begin{aligned}
& \hat{\omega}_{p}=\frac{\nu_{p}}{T_{\mathrm{samp}}} \\
& \hat{\theta}_{p}=\sin \left(\frac{\hat{\mu}_{p}^{\mathrm{r}}}{2 \pi d_{\mathrm{r}}}\right) \\
& \hat{\phi}_{p}=\sin \left(\frac{\hat{\mu}_{p}^{\mathrm{t}}}{2 \pi d_{\mathrm{t}}}\right)
\end{aligned}
$$


Using the estimates in (6.10) and (6.6), we form the $\hat{P}$ equations

$$
\left[\begin{array}{c}
\hat{\omega}_{1} \\
\hat{\omega}_{2} \\
\vdots \\
\hat{\omega}_{\hat{P}}
\end{array}\right]=\left[\begin{array}{cc}
\cos \left(\phi_{1}\right) & \cos \left(\theta_{1}\right) \\
\cos \left(\phi_{2}\right) & \cos \left(\theta_{2}\right) \\
\vdots & \vdots \\
\cos \left(\phi_{\hat{P}}\right) & \cos \left(\theta_{\hat{P}}\right)
\end{array}\right]\left[\begin{array}{l}
V_{\max }^{\mathrm{t}} \\
V_{\max }^{\mathrm{r}}
\end{array}\right]
$$

where $V_{\max }^{\mathrm{t}}$ and $V_{\max }^{\mathrm{r}}$ denote the maximum Doppler frequencies of the transmitter and receiver, respectively. Solving (6.11) yields estimates of the maximum Doppler frequencies as

$$
\hat{\mathbf{V}}_{\text {max }}=\left(\mathbf{E}^{H} \mathbf{E}\right)^{-1} \mathbf{E}^{H} \hat{\boldsymbol{\omega}}
$$

where $\hat{\boldsymbol{\omega}}=\left[\hat{\omega}_{1} \cdots, \hat{\omega}_{\hat{K}}\right]^{T}, \hat{\mathbf{V}}_{\max }=\left[\hat{V}_{\max }^{\mathrm{t}} \hat{V}_{\max }^{\mathrm{r}}\right]^{T}$ is a vector containing the Doppler frequencies and $\mathbf{E}$ is the $\hat{K} \times 2$ matrix in (6.11). The mobile velocities can then be calculated from the Doppler estimates using

$$
\begin{aligned}
& \hat{v}_{\mathrm{t}}=\frac{c \hat{V}_{\max }^{\mathrm{t}}}{2 \pi f_{\mathrm{c}}} \\
& \hat{v}_{\mathrm{r}}=\frac{c \hat{V}_{\max }^{\mathrm{r}}}{2 \pi f_{\mathrm{c}}}
\end{aligned}
$$

where $c$ is the speed of light and $f_{\mathrm{c}}$ is the carrier frequency.

\subsection{Numerical Simulations}

In this section, we evaluate the performance of the M-to-M prediction approach for different propagation scenarios. After a description of the simulation parameters in Section 6.4.1, the NMSE prediction performance is evaluated in Section 6.4.2. Finally, the performance of the velocity estimation method is discussed in Section 6.4.3.

\subsubsection{Simulation Parameters}

We consider a ULA at both the transmitter and the receiver. The carrier frequency for the transmission is $f_{\mathrm{c}}=2.0 \mathrm{GHz}$ and the velocities of the 
transmitter and receiver are, respectively, $v_{\mathrm{t}}=25 \mathrm{~km} / \mathrm{h}$ and $v_{\mathrm{r}}=50 \mathrm{~km} / \mathrm{h}$. The fading amplitudes $\beta_{p}$ are generated as complex Gaussian distributed random variables, $\beta_{p} \sim \mathcal{C N}(0,1)$. The angles of arrival and departure are assumed to be uniformly distributed i.e., $\theta_{p}, \phi_{p} \sim \mathcal{U}[-\pi, \pi)$. Except where otherwise stated, the prediction is based on $L=100$ samples with a sampling interval of $T_{\text {samp }}=2 \mathrm{~ms}$.

\subsubsection{Prediction Performance Evaluation}

We consider a scenario with $P=8$ paths $^{1}$. In Fig. 6.2, we plot the time varying amplitude and phase of the actual and predicted channel for the first entry of a $2 \times 2$ MIMO M-to-M system at SNR $=[5,20] \mathrm{dB}$. We observe that the proposed algorithm yields very good prediction of both the amplitude and phase of the channel at both SNR values except for instances of deep fades. We also observe that while the instant of deep fades and peaks are accurately predicted at $\mathrm{SNR}=20 \mathrm{~dB}$, the predicted deep fades at $5 \mathrm{~dB}$ have a small time lag $(<1 \mathrm{~ms})$ when compared to the actual channel. As expected, the predicted phase of the channel are also more accurate at $20 \mathrm{~dB}$. The prediction performance is quantified in Fig. 6.3 where we present the normalized mean square prediction error and error bound versus prediction horizon for a $2 \times 2$ MIMO channel at $\mathrm{SNR}=[5,20] \mathrm{dB}$. As expected, the prediction error increases with the prediction interval and decreases with SNR. We observe that the performance of the proposed method is closer to the bound at $\mathrm{SNR}=20 \mathrm{~dB}$. In Fig. 6.4, we show the CDF of the normalized squared prediction error at $\tau=10 \mathrm{~ms}$. The CDF curves clearly show that as SNR increases, the NMSE of the proposed algorithm decreases.

In Fig. 6.5, we plot the NMSE and NMSEB versus SNR for different numbers of antenna elements and prediction horizon of $\tau=10 \mathrm{~ms}$. We observe that increasing the number of transmit and receive antenna elements offers more information about the propagation channel and thus decreases the

\footnotetext{
${ }^{1}$ It has been shown in [27] that outdoor environments are characterized by few dominant scatterers and that the channel can be described by 3-8 distinct paths.
} 
NMSE at all SNR values for the prediction horizon considered, with a greater impact at low SNR. We also observe that for a $2 \times 2$ M-to-M channel, the performance of the proposed method approaches the lower bound as the SNR increases. The corresponding CDFs of normalized square error are shown in Fig. 6.6, which confirms the results for mean error values in Fig. 6.5.

The effects of the estimation length $\mathrm{L}$ on the prediction performance is shown in Fig. 6.7. We observe at $\mathrm{SNR}=10 \mathrm{~dB}, K=100$ is required to approach the NMSEB, with a much greater number needed at lower SNR. Approaching the NMSEB as the number of samples increases is expected since subspace based schemes are asymptotic maximum likelihood estimators. A similar observation is made in Fig. 6.8, where we present the CDF of normalized square error at $\tau=10 \mathrm{~ms}$ in . Finally, we illustrate the effect of transmitter and receiver velocity on the prediction NMSE at an interval of $\tau=10 \mathrm{~ms}$ in Fig. 6.9. It shows that as the transmitter and/or receiver velocity increases, the prediction NMSE decreases. This is intuitively satisfying since increased velocity results in longer spatial distance over the same training interval, thereby revealing more structure of the channel.

\subsubsection{Mobile Velocity Estimation Error}

The performance of the proposed mobile velocity estimation algorithm described in Section 6.3.1 is evaluated in terms of the root mean squared error (RMSE) defined as

$$
\operatorname{RMSE}\left(v_{\mathrm{t} / \mathrm{r}}\right)=\sqrt{\frac{1}{C} \sum_{c=1}^{C}\left(v_{\mathrm{t} / \mathrm{r}}-\hat{v}_{\mathrm{t} / \mathrm{r}}(c)\right)^{2}}
$$

where $C$ is the number of Monte Carlo simulations. In Figure 6.10, we plot the RMSE versus SNR for both the transmit and receive mobile velocity estimation and compare with the square root of the CRB on velocity estimates. As expected, the RMSE decreases and approaches the bound with increasing SNR. Finally, Figure 6.11 shows the effect of the number of propagation paths on the velocity estimation accuracy. As shown in the figure, 
increasing the number of paths, $P$ from 2 to 4 decreases the RMSE. However, the RMSE increases for $P>4$. A plausible explanation for this is that although an increase in the number of paths is expected to improve the accuracy of the least square velocity estimation method, the parameter estimates become less accurate as the number of paths increases. This shows that the performance of the velocity estimation stage is dependent on the accuracy of the channel parameter estimates with more accurate parameter estimates leading to improved velocity estimation accuracy.

Finally, we illustrate the effect of the transmitter and receiver velocity on the averaged RMSE performance in Fig. 6.12, where we plot the averaged velocity estimation RMSE versus SNR for different values of $v_{\mathrm{t}}$ and $v_{\mathrm{r}}$. We observe that an increase in velocity increases the RMSE at low SNR values. However, the RMSE values when normalized by the total velocity are of the same order of magnitude for all velocity values plotted.

\subsection{Summary}

In this chapter, we extended our narrowband prediction method to MIMO M-to-M wireless communication channels. Starting with a statistical model for M-to-M channels, we derived a parametrized model for jointly estimating the AOA, AOD and effective Doppler frequency shifts via a 3D extension of the ESPRIT algorithm as in the DOD/DOA-MEMCHAP. We proposed a simple and efficient scheme for the estimation of mobile velocities. Simulation results show that the M-to-M prediction method approaches the prediction error bound with increasing SNR and/or number of samples in the measured segment. 


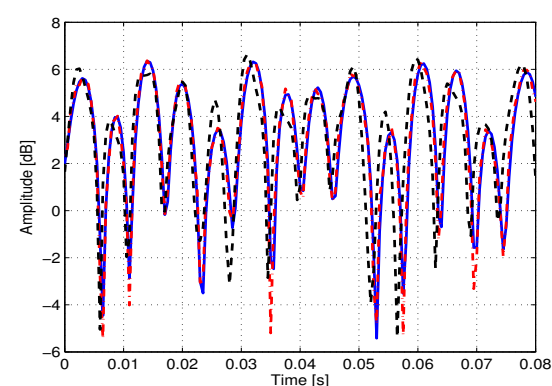

(a) Amplitude

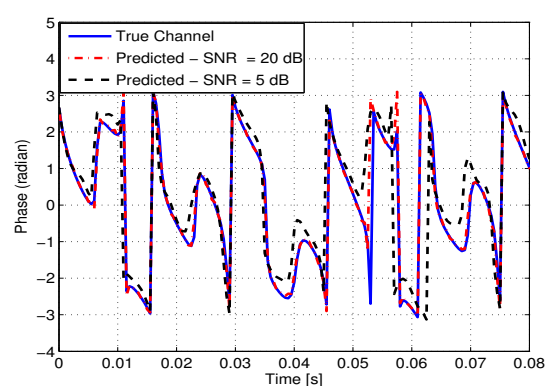

(b) Phase

Figure 6.2: A plot of the actual and predicted channel of a $2 \times 2$ MIMO M-to-M system at $\mathrm{SNR}=[5,20] \mathrm{dB}$.

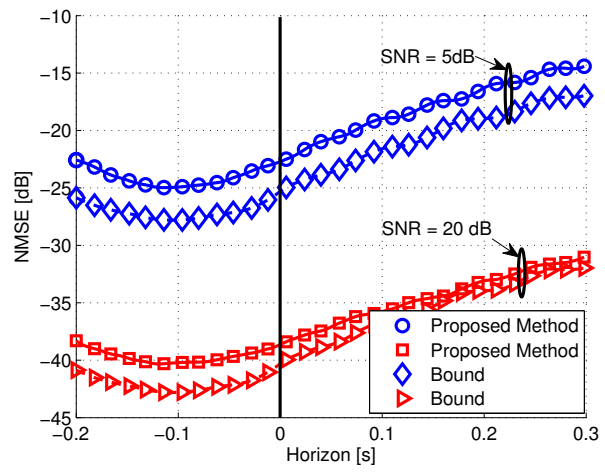

Figure 6.3: NMSE versus horizon.

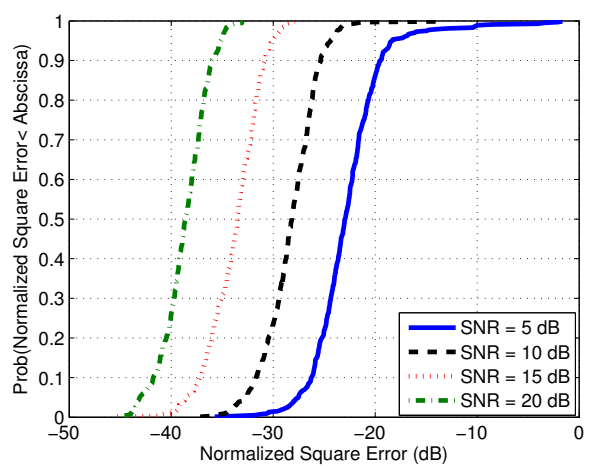

Figure 6.4: The CDF of NSE for a horizon of $10 \mathrm{~ms}$

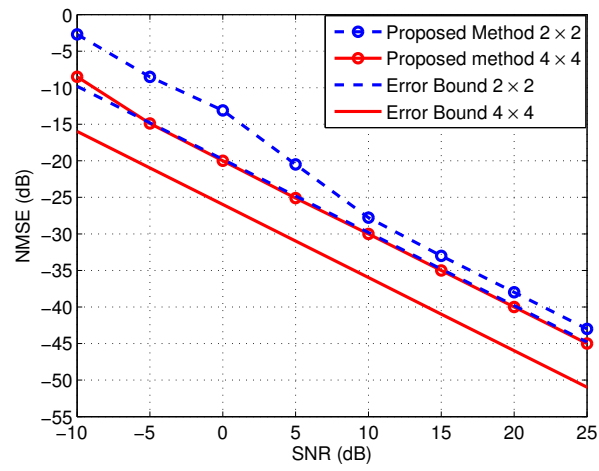

Figure 6.5: NMSE versus SNR.

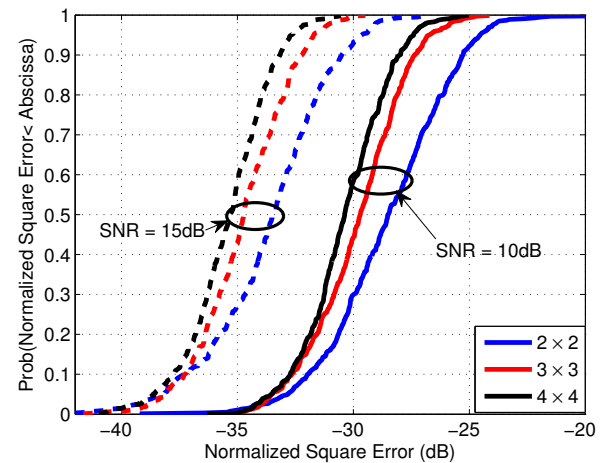

Figure 6.6: CDF of NSE for a horizon of $10 \mathrm{~ms}$ 


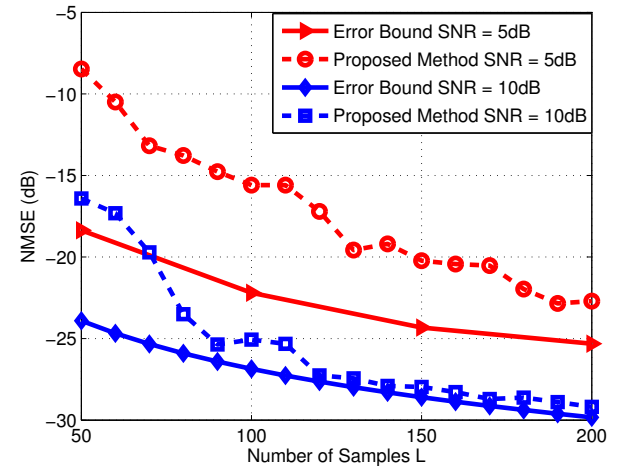

Figure 6.7: NMSE versus number of samples, K.

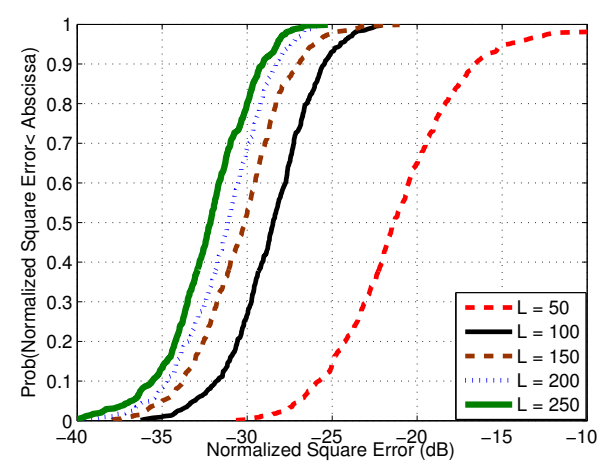

Figure 6.8: The CDF of NSE for a horizon of $\tau=10 \mathrm{~ms}$.

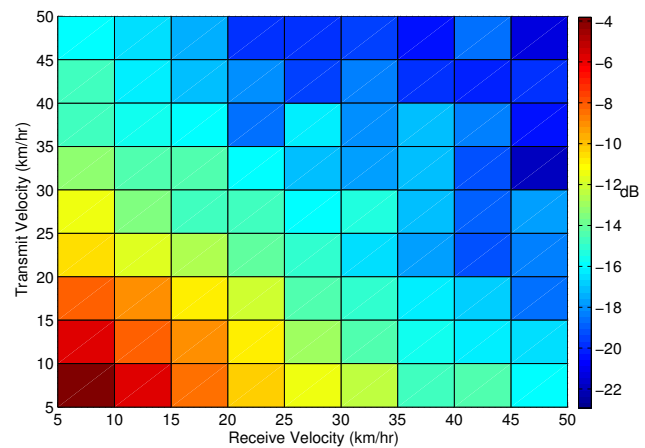

Figure 6.9: Effects of transmitter and receiver velocity on performance.

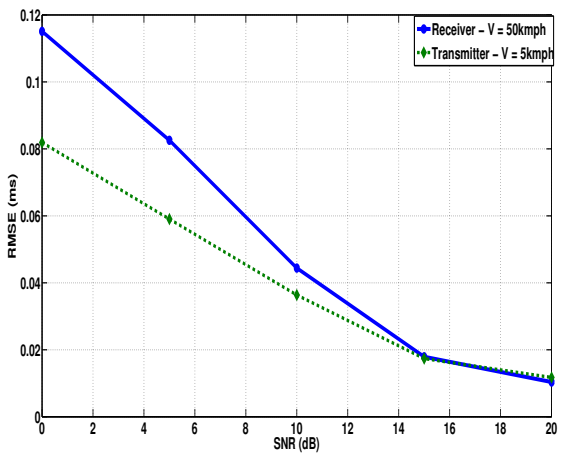

Figure 6.10: RMSE versus SNR with $K=2$.

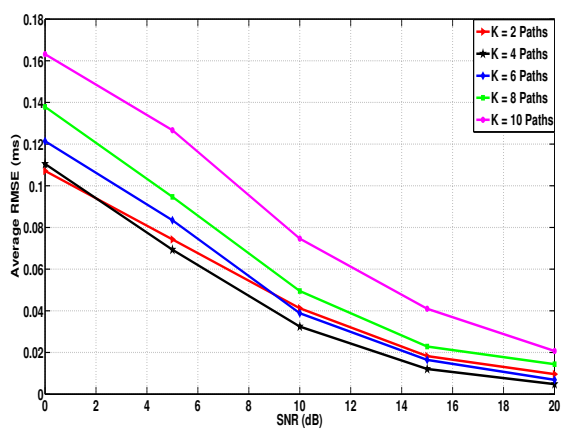

Figure 6.11: RMSE versus SNR.

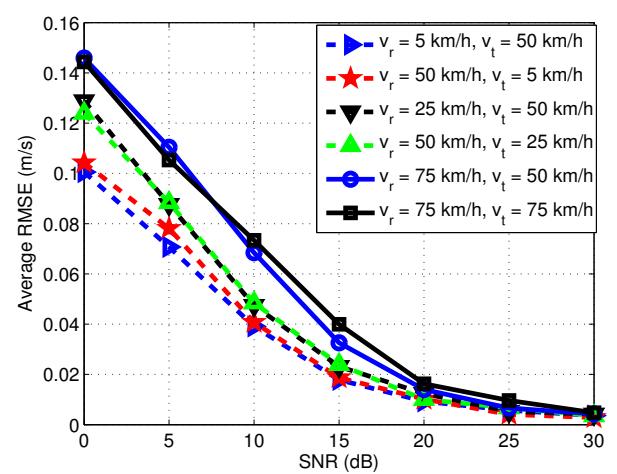

Figure 6.12: RMSE versus SNR for different values of $v_{r}$ and $v_{r}$.. 


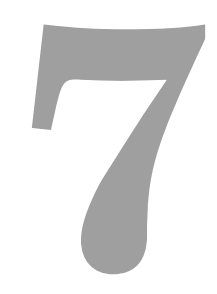

\title{
Application to Polarized MIMO
}

Channels

\begin{abstract}
dual polarized MIMO antenna configuration is a promising technique A for current and future wireless systems as it offers improved spectral efficiency (under the same spacing constraints) over single polarized MIMO. In this chapter we investigate the prediction of polarized narrowband MIMO channels and evaluate the benefits of polarization diversity [105] on the prediction performance. We extend our prediction concept of Chapters 4 and 5 to the extrapolation of narrowband and wideband MIMO channels with polarization diversity, respectively. The contents of this chapter have been presented in part in [17].
\end{abstract}

\subsection{Channel Models}

In this section, we present a brief review of the standardized WINNER II channel model and a parametrization of the model on which the parametric 


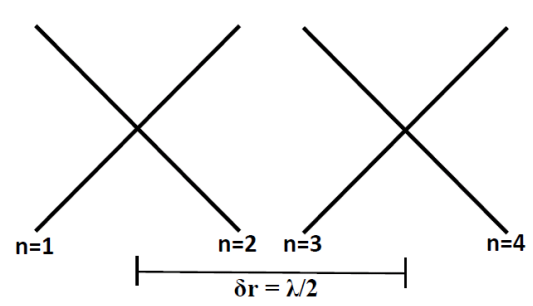

(a) \pm 45

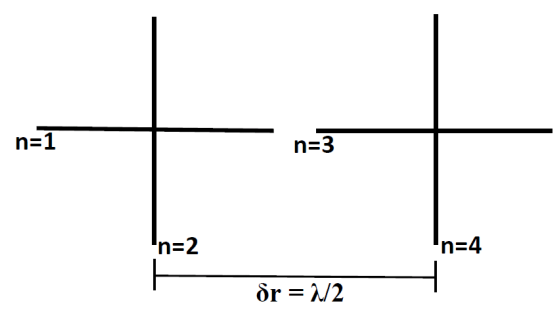

(b) $0 / \pi / 2$

Figure 7.1: Polarized MIMO Antenna Configurations: (a) Slanted 45 configuration and (b) VV/HH configuration.

prediction of the polarized channel is based.

\subsubsection{D Polarized MIMO Spatial Channel Model}

Consider a narrowband MIMO system as shown with a ULA of dual-polarized antenna pairs (see Fig. 7.1) at both the BS and MS. Assuming that the BS and MS arrays have $N$ and $M$ antenna pairs, respectively and that the propagation environment is characterized by $P$ stationary far field scatterers, the channel between the $n$th transmit and $m$ th receive antenna pair is given by [3]

$$
\begin{aligned}
h_{m, n}(t) & =\sum_{p=1}^{P}\left[\begin{array}{c}
\mathcal{X}_{\mathrm{t}, \mathrm{v}}^{n} \\
\mathcal{X}_{\mathrm{t}, \mathrm{h}}^{n}
\end{array}\right]^{H}\left[\begin{array}{cc}
e^{j \Phi_{p}^{\mathrm{vv}}} & \sqrt{\kappa_{p}} e^{j \Phi_{p}^{\mathrm{vh}}} \\
\sqrt{\kappa_{p}} e^{j \Phi_{p}^{\mathrm{hv}}} & e^{j \Phi_{p}^{\mathrm{hh}}}
\end{array}\right] \\
& \times\left[\begin{array}{c}
\mathcal{X}_{\mathrm{r}, \mathrm{v}}^{m} \\
\mathcal{X}_{\mathrm{r}, \mathrm{h}}^{m}
\end{array}\right] e^{j\left\{(n-1) \mu_{p}^{\mathrm{r}}+(m-1) \mu_{p}^{\mathrm{t}}+\omega_{p} t\right\}}
\end{aligned}
$$

where $\left[\Phi_{p}^{\mathrm{vv}}, \Phi_{p}^{\mathrm{vh}}, \Phi_{p}^{\mathrm{hv}}, \Phi_{p}^{\mathrm{hh}}\right] \sim U(-\pi, \pi)$ are the random initial phases of the $p$ th path for the four polarization components. $\mathcal{X}_{\mathrm{t}, \mathrm{v}}^{n}$ and $\mathcal{X}_{\mathrm{t}, \mathrm{h}}^{n}$ are the $n$th transmit antenna element field patterns for the vertical and horizontal polarizations, respectively. $\mathcal{X}_{\mathrm{r}, \mathrm{v}}^{m}$ and $\mathcal{X}_{\mathrm{r}, \mathrm{h}}^{m}$ are the $m$ th receive antenna element field patterns. The cross polarization discrimination (XPD) for the $p$ th path $\kappa_{p}=10^{X / 10}$, 
(where $X \sim \mathcal{N}(\sigma, u)$ ), is assumed to be log-Normal distributed ${ }^{1}$. $\omega_{p}=$ $\frac{v \cos \left(\theta_{p}-\vartheta_{v}\right)}{\lambda}$ is the Doppler shift of the $p$ th path. Finally, $v, \vartheta_{v}$ and $\lambda$ are the mobile speed, direction of motion and wavelength, respectively. If (for example) the BS and MS have ideal dipole antennas tilted at $\varrho$ from the vertical axis and the antenna polarization leakage effects were to be neglected, the antenna field pattern for the $p$ th path would be given by [171]

$$
\mathcal{X}_{R}=\left[\begin{array}{l}
\mathcal{X}_{R, v} \\
\mathcal{X}_{R, h}
\end{array}\right]=\left[\begin{array}{c}
\cos \varrho \\
\sin \varrho \cos \theta
\end{array}\right]
$$

\subsubsection{Parametrized Prediction Model}

In order to characterize the spatial structure and polarization diversity of the polarized MIMO channel, a matrix representation of the model in (7.1) is required. Collecting the $4 N M$ channels in (7.1) into a matrix, we obtain

$$
\mathbf{H}(t)=\left[\begin{array}{cccc}
h_{1,1}(t) & h_{2,1}(t) & \cdots & h_{2 N, 1}(t) \\
h_{2,1}(t) & h_{2,2}(t) & \cdots & h_{2 N, 2}(t) \\
\vdots & \vdots & \ddots & \vdots \\
h_{2 M, 1}(t) & h_{2 M, 2}(t) & \cdots & h_{2 M, 2 N}(t)
\end{array}\right]
$$

Note that each $2 \times 2$ submatrix of $\mathbf{H}(t)$ corresponds to the channel between one antenna pair at the receiver and another pair at the receiver. Using the model in (7.1), it can be shown that the $2 M \times 2 N$ polarized MIMO channel impulse response (7.3) is given by

$$
\mathbf{H}(t)=\sum_{p=1}^{P} \mathcal{S}_{\mathrm{t}}^{H}\left(\phi_{p}\right) \mathbf{G}_{p} \mathcal{S}_{\mathrm{r}}\left(\theta_{p}\right) e^{j \omega_{p} t}
$$

where

$$
\mathbf{G}_{p}=\left[\begin{array}{ll}
g_{p}^{\mathrm{vv}} & g_{p}^{\mathrm{vh}} \\
g_{p}^{\mathrm{hv}} & g_{p}^{\mathrm{hh}}
\end{array}\right] \in \mathbb{C}^{2 \times 2}
$$

\footnotetext{
${ }^{1}$ The path XPD can also be modeled as a distance dependent random variable as given in [59]. This dependence is however, not considered in this thesis.
} 
is the polarimetric weight matrix. $\mathcal{S}_{\mathrm{r}}\left(\theta_{p}\right) \in \mathbb{C}^{2 \times 2 M}$ is the receive polarized array steering matrix defined as

$$
\mathcal{S}_{\mathrm{r}}\left(\theta_{p}\right)=\mathbf{a}_{\mathrm{r}}\left(\theta_{p}\right) \otimes\left[\begin{array}{cc}
\cos \varrho_{1} & \cos \varrho_{2} \\
\sin \varrho_{1} \cos \theta_{p} & \sin \varrho_{2} \cos \theta_{p}
\end{array}\right]
$$

where $\varrho_{1}$ and $\varrho_{2}$ are the slant angles and $\left|\varrho_{1}-\varrho_{2}\right|=\pi / 2$ and the array steering vectors $\mathbf{a}_{\mathrm{r}}\left(\theta_{p}\right)$ and $\mathbf{a}_{\mathrm{t}}\left(\phi_{p}\right)$ are defined in (4.7) and (4.8), respectively. Assuming that the sampling interval is $T_{\text {samp }}$, the sampled channel at the $k$ time instant is thus

$$
\mathbf{H}(k)=\sum_{p=1}^{P} \mathcal{S}_{\mathrm{t}}^{H}\left(\phi_{p}\right) \mathbf{G}_{p} \mathcal{S}_{\mathrm{r}}\left(\theta_{p}\right) e^{j k \nu_{p}}
$$

As in the previous chapters, the parameters $\mathbf{G}_{p}, \theta_{\mathbf{p}}, \phi_{\mathbf{p}}$ and $\nu_{p}$ are assumed constant over the region of interest. We also assume that $K$ samples of the CSI matrix are available and we denote the estimated CSI matrix at time instant $k$ as

$$
\hat{\mathbf{H}}(k)=\mathbf{H}(k)+\mathbf{W}(k)
$$

where $\mathbf{W}(k) \in \mathbb{C}^{2 M \times 2 N}$ is a complex Gaussian random variable.

\subsubsection{D Polarized Wideband MIMO Model}

We consider a pilot based wideband MIMO system with ULA of dual-polarized antenna pairs at both the BS and mobile station (MS). We assume that the BS and MS arrays has $M$ and $N$ antenna pairs respectively and that the propagation environment is characterized by $P$ distinct paths each characterized by a set of quasi-static parameters, $\mathbf{\Theta}_{p}=\left[\mathbf{G}_{p}, \theta_{p}, \phi_{p}, \nu_{p}, \tau_{p}\right]$. Introducing the delay information into (7.4) yields

$$
\mathbf{H}(t, \tau)=\sum_{p=1}^{P} \mathcal{S}_{\mathrm{t}}^{H}\left(\phi_{p}\right) \mathbf{G}_{p} \mathcal{S}_{\mathrm{r}}\left(\theta_{p}\right) e^{j \omega_{p} t} \delta\left(\tau-\tau_{p}\right)
$$


The frequency response of the channel is obtained via a Fourier transformation of (7.9) as

$$
\mathbf{H}(t, f)=\sum_{p=1}^{P} \mathcal{S}_{\mathrm{t}}^{H}\left(\phi_{p}\right) \mathbf{G}_{p} \mathcal{S}_{\mathrm{r}}\left(\theta_{p}\right) e^{j\left\{\omega_{p} t-\omega \tau_{p}\right\}}
$$

Assuming that the system has adequate cyclic extension and symbol timing, the sampled frequency response at the $q$ symbol period for the $k$ th subcarrier is

$$
\mathbf{H}(q, k)=\sum_{p=1}^{P} \mathcal{S}_{\mathrm{t}}^{H}\left(\phi_{p}\right) \mathbf{G}_{p} \mathcal{S}_{\mathrm{r}}\left(\theta_{p}\right) e^{j\left\{q \nu_{p}-k \eta_{p}\right\}}
$$

where $\eta_{p}=\Delta f \omega_{p}$ is the normalized delay. It should be noted that $\nu_{p}$ and $\tau$ are upper bounded by $\nu_{\max }$ and $\tau_{\max }$ (i.e., $0 \leq \nu_{p}<\nu_{\max }$ and $0 \leq \tau_{p}<\tau_{\max }$ ) where $\nu_{\max }$ and $\tau_{\max }$ are the maximum Doppler shift an maximum delay, respectively.

\subsection{Polarized Narrowband MIMO Prediction}

Given the $K$ estimates of the channel and the model in (7.7), our aim is to estimate the parameters of the polarized MIMO channel and use the estimated parameters for the prediction of the CSI into the future. The different stages involved in the proposed prediction algorithm are presented in this section.

\subsubsection{Data Preprocessing and Covariance Matrix Esti- mation}

In order to jointly estimate the parameters of the channel using ESPRIT, we need to transform the CSI matrix such that the invariance structure requirement is satisfied. In a dual-polarized antenna configuration however, two elements in a pair occupy the same position and as such each multipath is received at the two elements without any delay/phase shift. The invariance 
structure is therefore only satisfied by corresponding elements of different pairs and not of the same pair. Consequently, the invariance structure is not satisfied. To progress we form four subsets (corresponding to the four polarization components) from the CSI matrix, given by

$$
\hat{\mathbf{D}}_{i j}(k)=\left[\begin{array}{cccc}
\hat{h}_{i, j}(k) & \hat{h}_{i, j+2}(k) & \cdots & \hat{h}_{i, m_{j}}(k) \\
\hat{h}_{i+2, j}(k) & \hat{h}_{i+2, j+2}(k) & \cdots & \hat{h}_{i+2, m_{j}}(k) \\
\vdots & \vdots & \ddots & \vdots \\
\hat{h}_{n_{i}, j}(k) & \hat{h}_{n_{i}, j+2}(k) & \cdots & \hat{h}_{n_{i}, m_{j}}(k)
\end{array}\right]
$$

where $\hat{\mathbf{h}}_{i, j}(k)$ is the $(i, j)$ th entry of $\hat{\mathbf{H}}(k), n_{i}=(2 N+i-2)$ and $m_{j}=$ $(2 M+j-2)$ and $i, j=1,2$. The submatrices in (7.12) correspond to the four possible polarization combinations in a dual polarized MIMO channel. This is to ensure that entries of the submatrices are phase shifted versions of each other as required for parameter extraction using ESPRIT [158]. Letting $\hat{\mathbf{d}}_{i j}(k)=\operatorname{vec}\left[\hat{\mathbf{D}}_{i j}(k)\right]$ be the vectorized form of $\hat{\mathbf{D}}_{i j}(k)$, obtained by stacking its column, we form an $N M R \times L$ Hankel matrix for each subset denoted by

$$
\hat{\mathbf{Q}}_{i j}=\left[\begin{array}{cccc}
\hat{\mathbf{d}}_{i j}(1) & \hat{\mathbf{d}}_{i j}(2) & \cdots & \hat{\mathbf{d}}_{i j}(L) \\
\hat{\mathbf{d}}_{i j}(2) & \hat{\mathbf{d}}_{i j}(3) & \cdots & \hat{\mathbf{d}}_{i j}(L+1) \\
\vdots & \vdots & \ddots & \vdots \\
\hat{\mathbf{d}}_{i j}(R) & \hat{\mathbf{d}}_{i j}(R+1) & \cdots & \hat{\mathbf{d}}_{i j}(K)
\end{array}\right] i, j=1,2
$$

where $L=K-R+1$ and $R$ is chosen such that $N M R \geq P+1$. The data in $\hat{\mathbf{Q}}_{i j}$ is equivalent to $L$ observations from an $N \times M \times R$ three dimensional antenna array. It should however be noted that the data is obtained by combining the receive spatial, temporal and transmit spatial samples of the channel. The spatio-temporal covariance matrix averaged over the four polarization components is then estimated using ${ }^{2}$

$$
\hat{\mathbf{C}}=\frac{1}{4 L} \sum_{i=1}^{2} \sum_{j=1}^{2}\left(\hat{\mathbf{Q}}_{i j} \hat{\mathbf{Q}}_{i j}^{H}\right)
$$

\footnotetext{
${ }^{2}$ Averaging of the covariance matrix over the polarization combinations results in a better estimate of the covariance matrix and hence parameter estimates.
} 
This matrix includes the covariance between every possible pair of transmit antenna, temporal virtual array (resulting from the transformation in (7.13)) antenna element and receive antenna.

\subsubsection{Subspace Dimension Estimation}

As in the previous chapters, we estimate the number of dominant paths using the MMDL criterion. Let $\hat{\lambda}_{1} \geq \hat{\lambda}_{2} \geq \cdots \geq \hat{\lambda}_{N M R}$ be the eigenvalues of $\hat{\mathbf{C}}$ in descending order of magnitude, and $\hat{\mathbf{e}}_{1}, \hat{\mathbf{e}}_{2}, \cdots, \hat{\mathbf{e}}_{N M R}$ the associated eigenvectors. The estimate of the number of paths is obtained from

$$
\hat{P}=\arg \min _{p=1, \cdots, N M R-1} \operatorname{MMDL}(p)
$$

where the $\operatorname{MMSE-MDL}$ criterion $\operatorname{MMDL}(p)$ is defined as [85]

$$
\operatorname{MMDL}(p)=L \log \left(\lambda_{p}\right)+\frac{1}{2}\left(p^{2}+p\right) \log L
$$

\subsubsection{Joint Parameter Estimation}

We arrange the $\hat{P}$ eigenvectors corresponding to the largest eigenvalues of $\hat{\mathbf{C}}$ into a matrix $\mathbf{E}=\left[\hat{\mathbf{e}}_{1}, \cdots, \hat{\mathbf{e}}_{\hat{P}}\right]$ and form the following invariance equations ${ }^{3}$

$$
\begin{aligned}
\mathbf{J}_{\mathrm{r} 2} \mathbf{E} & =\mathbf{J}_{\mathrm{r} 1} \mathbf{E} \boldsymbol{\Phi}_{\mathrm{r}} \\
\mathbf{J}_{\mathrm{t} 2} \mathbf{E} & =\mathbf{J}_{\mathrm{t} 1} \mathbf{E} \boldsymbol{\Phi}_{\mathrm{t}} \\
\mathbf{J}_{\mathrm{d} 2} \mathbf{E} & =\mathbf{J}_{\mathrm{d} 1} \mathbf{E} \boldsymbol{\Phi}_{\mathrm{d}}
\end{aligned}
$$

where $\boldsymbol{\Phi}_{\mathrm{r}}, \boldsymbol{\Phi}_{\mathrm{t}}$ and $\boldsymbol{\Phi}_{\mathrm{d}}$ are subspace rotation matrices which are related to the channels parameters as in (4.82) and $\mathbf{J}_{x i} ; i=1,2$ are the selection matrices defined analogous to (4.97). The equations in (7.17) are then solved in a

\footnotetext{
${ }^{3}$ These equations are analogous to the 1-D ESPRIT equation $\mathbf{S}_{1} \mathbf{E}=\mathbf{S}_{2} \mathbf{E} \mathbf{\Phi}$ [158] where $\mathbf{S}_{1}$ and $\mathbf{S}_{2}$ remove the first and last rows of $\mathbf{E}$ respectively and $\mathbf{\Phi}$ is the 1-D subspace rotation matrix.
} 
least square sense to obtain estimates of the rotation matrices. The MEVD is then used to enable automatic pairing of the estimates thus

$$
\begin{aligned}
\boldsymbol{\Phi} & =\boldsymbol{\Phi}_{\mathrm{r}}+\boldsymbol{\Phi}_{\mathrm{t}}+\boldsymbol{\Phi}_{\mathrm{d}} \\
& =\mathbf{T} \boldsymbol{\Lambda} \mathbf{T}^{-1}
\end{aligned}
$$

where $\mathbf{T}$ is the common eigenvector matrix and $\boldsymbol{\Lambda}$ is a diagonal matrix containing the eigenvalues of $\boldsymbol{\Phi}$. The $\hat{P}$ eigenvalues for each dimension are then computed using

$$
\begin{aligned}
& \boldsymbol{\Lambda}_{\mathrm{r}}=\operatorname{diag}\left[\mathbf{T}^{-1} \boldsymbol{\Phi}_{\mathrm{r}} \mathbf{T}\right] \\
& \boldsymbol{\Lambda}_{\mathrm{t}}=\operatorname{diag}\left[\mathbf{T}^{-1} \boldsymbol{\Phi}_{\mathrm{t}} \mathbf{T}\right] \\
& \boldsymbol{\Lambda}_{\mathrm{d}}=\operatorname{diag}\left[\mathbf{T}^{-1} \boldsymbol{\Phi}_{\mathrm{d}} \mathbf{T}\right]
\end{aligned}
$$

The parameter estimates for the $p$ th path are given by

$$
\begin{aligned}
& \hat{\theta}_{p}=\operatorname{asin}\left(\frac{-\arg \left[\boldsymbol{\Lambda}_{\mathrm{r}}(p)\right]}{2 \pi \delta r}\right) \\
& \hat{\phi}_{p}=\operatorname{asin}\left(\frac{\arg \left[\boldsymbol{\Lambda}_{\mathrm{t}}(p)\right]}{2 \pi \delta t}\right) \\
& \hat{\nu}_{p}=\arg \left[\boldsymbol{\Lambda}_{\mathrm{d}}(p)\right]
\end{aligned}
$$

\subsubsection{Polarimetric Weights Estimation}

We assume that the complex polarimetric weights for all antenna pairs are equal. This assumption is reasonable considering the separation of polarization effects from path attenuation in (7.1) and the small spacing between antenna elements. In order to derive a model for the weight estimation problem, we divide the channel matrix into $2 \times 2$ sub-matrices as follows

$$
\hat{\mathbf{H}}_{\mathrm{sub}}^{n m}(k)=\left[\begin{array}{cc}
\hat{h}_{2 n-1,2 m-1}(k) & \hat{h}_{2 n-1,2 m}(k) \\
\hat{h}_{2 n, 2 m-1}(k) & \hat{h}_{2 n, 2 m}(k)
\end{array}\right]
$$

For clarity reasons, we first derive a model for the first sub-matrix followed by a generalization to the other submatrices. Using (7.21), the first $2 \times 2$ 
submatrix of the channel matrix is obtained as

$$
\hat{\mathbf{H}}_{\text {sub }}^{11}(k)=\left[\begin{array}{ll}
\hat{h}_{1,1}(k) & \hat{h}_{1,2}(k) \\
\hat{h}_{2,1}(k) & \hat{h}_{2,2}(k)
\end{array}\right]
$$

It can be shown from (7.1) that the entries of $\hat{\mathbf{H}}_{\text {sub }}^{11}$ are defined as

$$
\hat{h}_{i, j}(k)=\sum_{p=1}^{P}\left[\begin{array}{c}
\cos \varrho_{i} \cos \varrho_{j} \\
\cos \varrho_{i} \sin \varrho_{j} \cos \phi_{p} \\
\cos \varrho_{i} \sin \varrho_{j} \cos \theta_{p} \\
\sin \varrho_{i} \sin \varrho_{i} \cos \theta_{p} \cos \phi_{p}
\end{array}\right]^{T}\left[\begin{array}{l}
g_{p}^{v v} \\
g_{p}^{h v} \\
g_{p}^{v h} \\
g_{p}^{h h}
\end{array}\right] e^{j(k-1) \nu_{p}}+w(k)
$$

This can be expressed as

$$
\hat{h}_{i, j}(k)=\sum_{p=1}^{P} \mathbf{u}_{p}^{i j} \mathbf{g}_{p} e^{j(k-1) \nu_{p}}+w(k)
$$

The definitions of $\mathbf{u}_{p}^{i j}$ follows from (7.23). Collecting the $K$ known samples into a vector $\hat{\mathbf{h}}_{i, j}=\left[\hat{h}_{i, j}(1), \cdots, \hat{h}_{i, j}(K)\right]^{T}$, we obtain

$$
\hat{\mathbf{h}}_{i j}=\sum_{p=1}^{P}\left(\mathbf{f}_{p} \otimes \mathbf{u}_{p}^{i j}\right) \mathbf{g}_{p}+\mathbf{w}
$$

where $\mathbf{f}_{p}=\left[\begin{array}{llll}1 & e^{j \nu_{p}} & \cdots & e^{j(K-1) \nu_{p}}\end{array}\right]^{T}$ and $\mathbf{w}$ is the noise vector. A matrix representation for (7.25) is thus

$$
\hat{\mathbf{h}}_{i j}=\mathbf{F}_{i j} \mathbf{g}+\mathbf{w}_{i j} \quad i, j=1,2
$$

where $\mathbf{F}_{i j}=\left[\mathbf{f}_{1} \otimes \mathbf{u}_{1}^{i j}, \cdots, \mathbf{f}_{P} \otimes \mathbf{u}_{P}^{i j}\right] \in \mathbb{C}^{K \times 4 \hat{P}}$ and $\mathbf{g}=\left[\mathbf{g}_{1}^{T} ; \cdots ; \mathbf{g}_{\hat{P}}^{T}\right] \in \mathbb{C}^{4 \hat{P} \times 1}$ is a vector containing the polarimetric weights for all scattering sources. We combine the four equations in (7.26) to obtain

$$
\mathbf{h}=\mathbf{F g}+\mathbf{w}
$$

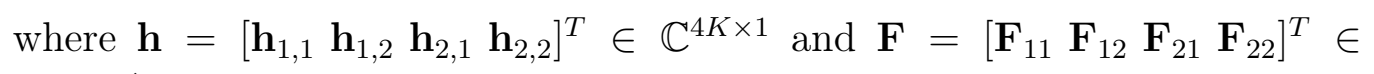
$\mathbb{C}^{4 K \times 4 \hat{P}}$ and solve (7.27) using regularized least squares to obtain estimates of the polarimetric weights

$$
\hat{\mathrm{g}}=\left(\mathbf{F}^{H} \mathbf{F}+\eta \mathbf{I}\right)^{-1} \mathbf{F}^{H} \mathbf{h}
$$


where $\eta$ is a regularizing parameter that improves the robustness of the predictor by reducing sensitivity to errors in the estimation of $\mathbf{F}$. As in the previous chapters, $\eta$ was chosen empirically to be $10^{-5}$ in this chapter. It should be noted that for complexity reasons, we utilize only a submatrix of the entire channel matrix for estimating the complex weights in this this chapter, the entire matrix can be utilized for the estimation as in Section 4.4.4. The complexity of the complex weight estimation stage will increase by an order of $N M / 2$.

\subsubsection{CSI Prediction}

Once the parameters of the channel are estimated, the extrapolation of the polarized MIMO CSI is achieved by substituting the estimated multipath parameters into the model for the desired prediction horizon. The predicted CSI is thus

$$
\mathbf{H}(k)=\sum_{p=1}^{\hat{P}} \mathcal{S}_{\mathrm{t}}^{H}\left(\hat{\phi}_{p}\right) \hat{\mathbf{G}}_{p} \mathcal{S}_{\mathrm{r}}\left(\hat{\theta}_{p}\right) e^{j k \hat{\nu}_{p}}
$$

\subsection{Polarized Wideband MIMO Prediction}

In Section 7.2, we presented a scheme for the extrapolation of narrowband MIMO channels with polarized antenna arrays. A similar approach for wideband polarized channels is derived in this section. Since the source number and parameter estimation stages are similar to the DOD/DOAWIMEMCHAP in Section 5.3.1, we only summarized the data transformation, polarimetric weights estimation and CSI prediction stages in this section. 


\subsubsection{Data Transformation}

Analogous to (7.12), we define CSI submatrices for each polarization pair thus

$$
\hat{\mathbf{D}}_{i j}(q, k)=\left[\begin{array}{cccc}
\hat{h}_{i, j}(q, k) & \hat{h}_{i, j+2}(q, k) & \cdots & \hat{h}_{i, m_{j}}(q, k) \\
\hat{h}_{i+2, j}(q, k) & \hat{h}_{i+2, j+2}(q, k) & \cdots & \hat{h}_{i+2, m_{j}}(q, k) \\
\vdots & \vdots & \ddots & \vdots \\
\hat{h}_{n_{i}, j}(q, k) & \hat{h}_{n_{i}, j+2}(q, k) & \cdots & \hat{h}_{n_{i}, m_{j}}(q, k)
\end{array}\right]
$$

Letting $\hat{\mathbf{d}}(q, k)=\operatorname{vec}\left[\hat{\mathbf{D}}_{i j}(q, k)\right]$, we form an $N M R \times L$ Hankel matrix for each subset at every frequency location

$$
\hat{\mathbf{Q}}_{i j}(q)=\left[\begin{array}{cccc}
\hat{\mathbf{d}}_{i j}(1, q) & \hat{\mathbf{d}}_{i j}(2, q) & \cdots & \hat{\mathbf{d}}_{i j}(L, q) \\
\hat{\mathbf{d}}_{i j}(2, q) & \hat{\mathbf{d}}_{i j}(3, q) & \cdots & \hat{\mathbf{d}}_{i j}(L+1, q) \\
\vdots & \vdots & \ddots & \vdots \\
\hat{\mathbf{d}}_{i j}(R, q) & \hat{\mathbf{d}}_{i j}(R+1, q) & \cdots & \hat{\mathbf{d}}_{i j}(K, q)
\end{array}\right] i, j=1,2
$$

where $L=K-R+1$. The data in $\hat{\mathbf{Q}}_{i j}(q)$ corresponds to $L$ observations from a three dimensional array. It should however be noted that the data is obtained by combining the receive and transmit spatial correlation and temporal structures of the channel. The frequency structure is introduced by forming a $N M R V \times L U$ block Hankel matrix from (7.31) as

$$
\hat{\mathbf{X}}_{i j}=\left[\begin{array}{cccc}
\hat{\mathbf{Q}}_{i j}(1) & \hat{\mathbf{Q}}_{i j}(2) & \cdots & \hat{\mathbf{Q}}_{i j}(U) \\
\hat{\mathbf{Q}}_{i j}(2) & \hat{\mathbf{Q}}_{i j}(3) & \ldots & \hat{\mathbf{Q}}_{i j}(U+1) \\
\vdots & \vdots & \ddots & \vdots \\
\hat{\mathbf{Q}}_{i j}(V) & \hat{\mathbf{Q}}_{i j}(V+1) & \cdots & \hat{\mathbf{Q}}_{i j}(Q)
\end{array}\right] i=1,2
$$

\subsubsection{Covariance Matrix Estimation}

The estimate of the covariance matrix is defined as

$$
\hat{\mathbf{C}}=\frac{1}{4 L U} \sum_{i=1}^{2} \sum_{j=1}^{2}\left(\hat{\mathbf{X}}_{i j} \hat{\mathbf{X}}_{i j}^{H}\right)
$$




\subsubsection{ESPRIT Based Joint Parameter Estimation}

After estimating the number of paths, $P$, using the MMDL criterion, the spatial frequencies $\mu^{\mathrm{r}}$, and $\mu^{\mathrm{r}}$, as well as the normalized Doppler frequencies and delays are estimated by solving (5.30)-(5.44). Estimates of the AOA and AOD are then obtained from the corresponding spatial frequency using

$$
\begin{aligned}
& \theta_{p}=\operatorname{asin}\left(\frac{\boldsymbol{\mu}^{\mathrm{r}}(p)}{2 \pi \delta r}\right) \\
& \phi_{p}=\operatorname{asin}\left(\frac{\boldsymbol{\mu}^{\mathrm{t}}(p)}{2 \pi \delta t}\right)
\end{aligned}
$$

\subsubsection{Polarimetric Weight Estimation and CSI Predic- tion}

We assume that the complex polarimetric weights for all transmit and receive antenna pairs at all subcarriers are equal. The complex weight matrices can therefore be estimated from the first $2 \times 2$ submatrix of the channel transfer function at the first pilot subcarrier following a similar procedure as presented in Section 7.2.4. The doubly selective polarized channel is then predicted by substituting the estimated parameters into (7.11).

\subsection{Numerical Simulation}

The performance of the polarized prediction is evaluated in this section, and compared with error bound derived in Section 8.2. We consider a narrowband system with parameters shown in Table 7.1. As in the previous chapters, the NMSE is used as the performance metric. In Figure 7.2, we present the NMSE as a function of the prediction horizon at different SNR values. As expected, the performance improves with increasing SNR and the NMSE is closer to the error bound at higher SNR values. The corresponding CDF of NSE at a prediction interval of $2 \lambda$ is shown in Figure 7.3. 
Table 7.1: Simulation Parameters

\begin{tabular}{cc}
\hline Parameter & Value \\
\hline Number of antenna pairs (BS,MS) & $N / M=2,4$ \\
BS antenna spacing & $1 / 2 \lambda$ \\
MS antenna spacing & $1 / 2 \lambda$ \\
Number of Sources & 6 \\
Carrier frequency & $2.1 \mathrm{GHz}$ \\
Mobile Velocity & $50 \mathrm{~km} / \mathrm{h}$ \\
AOD & $U[0,2 \pi)$ \\
AOA & $U[0,2 \pi)$ \\
Sampling Interval & $2 \mathrm{~ms}$ \\
Training length & 50,100 \\
Dual Polarized Antenna Orientation & $\pm \pi / 4,0 / \pi / 2$ \\
\hline
\end{tabular}

The effect of noise and number of antenna pairs on the prediction performance is shown in Figure 7.4, where we plot the NMSE and error bound for a prediction horizon of $1 \lambda$ as a function of SNR. The NMSE decreases with increasing SNR and/or number of transmit and receive antenna pairs. We observe that, for the $2 \times 2$ system, the NMSE approaches the bound closely at high SNR. Figure 7.5 presents the CDF of NSE for a prediction horizon of $1 \lambda$. It also shows that increased SNR and/or number of antenna pairs improves the performance.

In Figure 7.6, we plot the NMSE versus number of samples, $K$ in the training segment for different prediction horizons. We observe that the NMSE decreases with increasing number of samples. However, no significant decrease is observed for $K \geq 150$. A similar trend is observed in Figure 7.7, where we present the CDF of NSE for different values of $K$.

Finally, we illustrate the effect of antenna orientation (slant angles) on the prediction performance in Figures 7.8-7.9. In Figure 7.8, we plot the NMSE as a function of prediction horizon for antenna slant angles of $\pm \pi / 4$ and $0, \pi / 2$. We observe that the $0, \pi / 2$ configuration offers better performance 
when compared to the $\pm \pi / 4$. A plausible explanation for this is that the information at the elements of an antenna pair in the $\pm \pi / 4$ configuration are more correlated compared to the $0, \pi / 2$ and as such provide less additional information. A similar observation is made in Figure 7.9 where we plot the CDF for a prediction length of $1 \lambda$.

\subsection{Conclusion}

We have extended the prediction schemes in Chapters 4 and 5 to polarized narrowband and wideband MIMO channels. The additional information offered by the polarization diversity is incorporated into the schemes to improve parameter estimation and CSI prediction accuracy. The performance of the polarized CSI prediction scheme is compared to the error bound (see Section 8.2 for derivation of the error bound.) and results shows that the performance of the scheme approaches the bound with increasing SNR and/or training length. The antenna orientation also affects the performance with antennas oriented at $0, \pi / 2$ offering better performance than those with $\pm \pi / 4$ slant angles. It should be noted that although the $0, \pi / 2$ antenna orientation offer better prediction performance, more study may be neccessary to determine its suitabilty for polarization diversity since current commercial practices only use the $\pm \pi / 4$ configuration. 


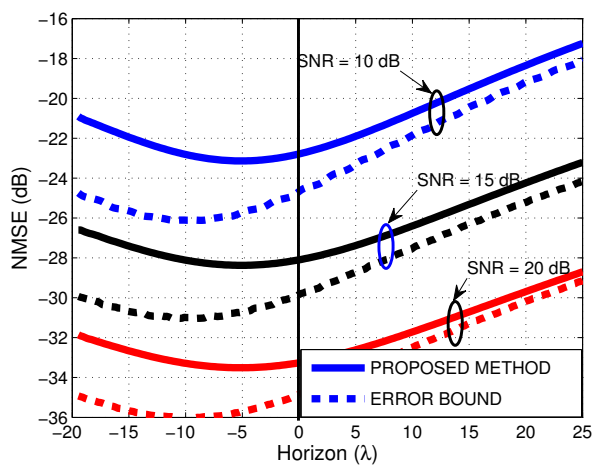

Figure 7.2: NMSE versus horizon for a $\pm \pi / 42 \times 2$ channel.

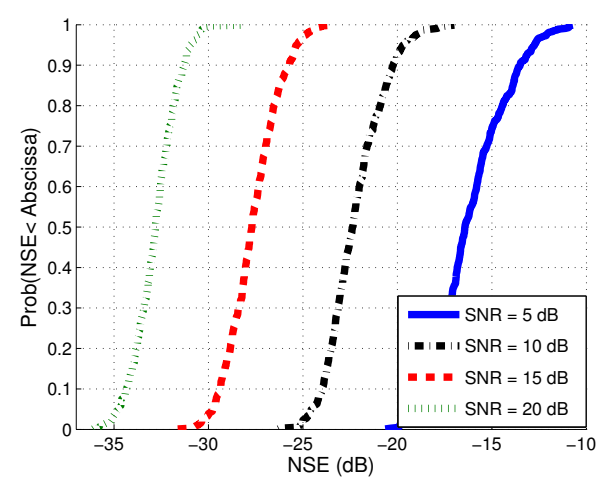

Figure 7.3: The CDF of NSE for a horizon of $1 \lambda$.

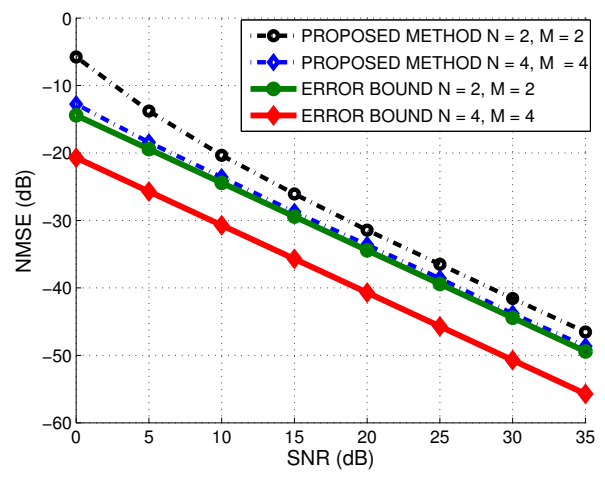

Figure 7.4: Effects of SNR on NMSE for $\varrho= \pm \pi / 4$.

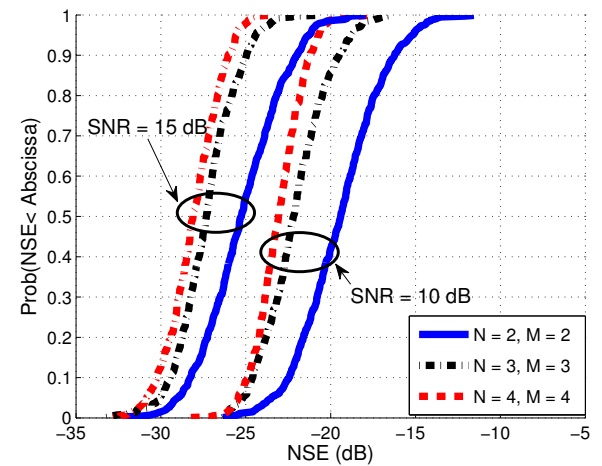

Figure 7.5: NSE CDF for varying number of antenna elements.

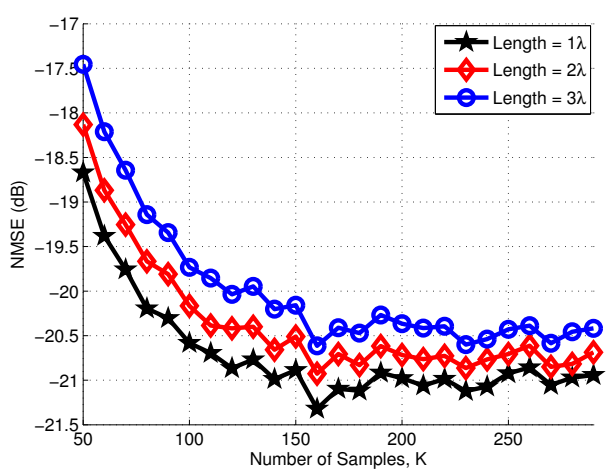

Figure 7.6: Effects of number of samples, $\mathrm{K}$ on the NMSE at $\mathrm{SNR}=10 \mathrm{~dB}$.

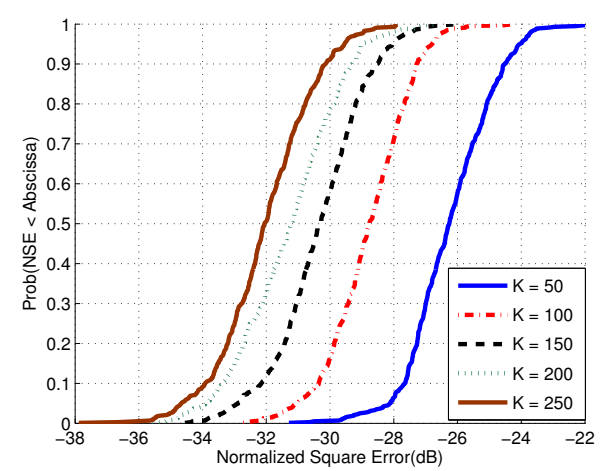

Figure 7.7: CDF of NSE for different number of samples. 


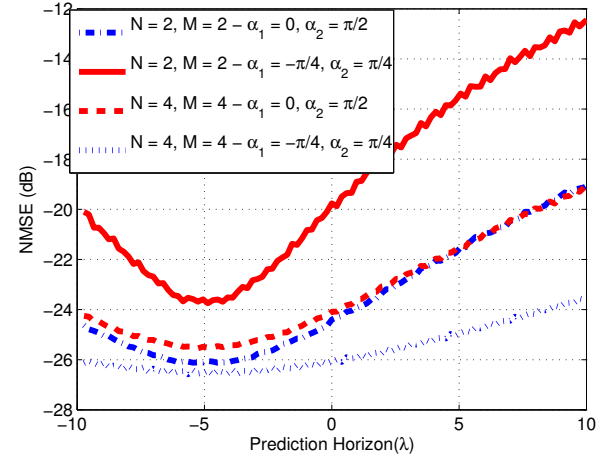

Figure 7.8: Effects of antenna slant Figure 7.9: CDF of NSE at SNR= angles on NMSE.

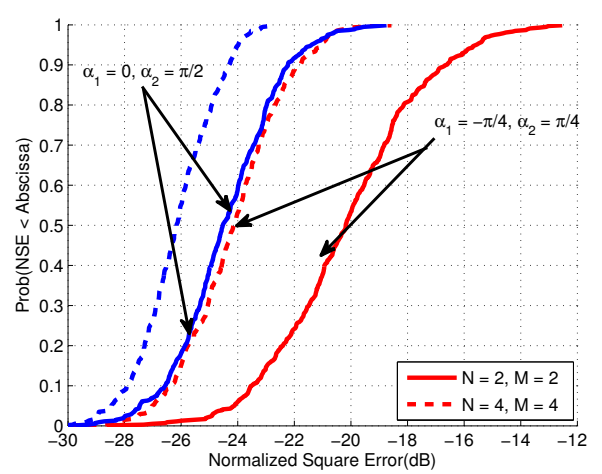

$15 \mathrm{~dB}$ with different slant angles. 


\section{8}

\section{Bounds on the Prediction of Mobile MIMO Channels}

$\left[\begin{array}{l}\mathrm{N} \text { the previous chapters, we have developed schemes for the prediction of } \\ \text { narrowband as well as wideband MIMO wireless channels. Extensions }\end{array}\right.$ to polarized MIMO channels and Mobile-to-mobile (M-to-M) systems have also been presented. However, to the authors' knowledge, there exists no closed form expression that relates CSI prediction error to predictor design parameters such as number of antennas, number of samples in the observation segment, number of paths and SNR.

In $[35,190]$, bounds on the prediction error in SISO channels were derived. The authors of [205] derived an asymptotic error bound on the prediction of SISO-OFDM channels. Bounds on the prediction of narrowband MIMO channels were studied in [189]. Bounds on the interpolation of MIMO-OFDM channels were derived in [108] using a vector formulation of the CramerRao bound for a function of parameters. Similar bounds for estimation and prediction were proposed in $[109,110]$. Although these bounds are useful 
in their own way, their expressions are not easily interpretable. Moreover, their dependence on channel parameters necessitates averaging over several realizations of the channel and this reduces their interpretability.

Motivated by the need for simple, insightful and easily interpretable closed form expressions relating prediction error to algorithm design parameters, and the benefits of having bounds upon which the performance of any predictor can be compared, we make the following contributions in this chapter:

- Using the vector formulation of the Cramer Rao bound for functions of parameters [97] along with the properties of Kronecker products, we derive expressions for bounds on the prediction of narrowband MIMO channels with uniform linear arrays (ULA) at both ends of the link. Similar expressions are derived for pilot-based wideband MIMO systems, polarized narrowband and wideband MIMO systems and 3D propagations. The formulations obtained are simpler and provide an alternative for easier calculation compared to the results of $[109,110,189]$.

- We derive a simple closed-form expression for the best achievable mean square error (MSE) and normalized mean square error (NMSE) for an unbiased joint estimator and predictor in the asymptotic limit of large samples and/or many antenna elements. The derived expression provides useful insights into the development of algorithms for narrowband MIMO channel prediction. Also, closed-form expressions are derived for wideband channels and 3D propagation.

- Simulation results show that the asymptotic bound offers a very good approximation to the bound while eliminating the need for repeated computation and dependence on channel parameters.

The bounds are applicable to pilot based channel estimation, interpolation and prediction. The dependence of these bounds on system parameters, but not on channel parameters, enables them to provide useful insight into system design considerations. The content of the chapter have been published/submitted to $[11,12,15,18,19]$. 


\subsection{MIMO Channel Prediction Error Bound}

The prediction error bounds on narrowband MIMO channels as well as wideband and 3D channels are presented in this section.

\subsubsection{Narrowband Channel}

In this section, we derive the lower bound on the prediction MSE for narrowband channels. Although a similar error bound has been derived in [189], we utilize the properties of Khatri-Rao product to present an alternative, simplified derivation and expression for the FIM. Our simplified formulation provide the same results for the error bounds as the expressions in [189].

\section{Channel Model}

We consider the ray-based channel model defined for a MIMO channel at the $k$ th sampling interval: ${ }^{1}$

$$
\mathbf{H}(k)=\sum_{p=1}^{P} \alpha_{\mathrm{p}} \mathbf{a}_{\mathrm{r}}\left(\mu_{p}^{\mathrm{r}}\right) \mathbf{a}_{\mathrm{t}}^{T}\left(\mu_{p}^{\mathrm{t}}\right) e^{j k \nu_{p}}
$$

where $P$ is the number of paths, and $\alpha_{p}$ and $\nu_{p}$ are the complex amplitude and normalized radian Doppler frequency of the $p$ th path. The receive and transmit array response vectors associated with the $p$ th path are denoted by $\mathbf{a}_{\mathrm{r}}\left(\mu_{p}^{\mathrm{r}}\right)$ and $\mathbf{a}_{\mathrm{t}}\left(\mu_{p}^{\mathrm{t}}\right)$, respectively, where $\mu_{p}^{\mathrm{r}}$ and $\mu_{p}^{\mathrm{t}}$ are the angular frequencies associated with the directions of arrival and departure of the $p$ th path, respectively. Note that while (8.1) is valid for all antenna geometries, we will consider a ULA such that $\mathbf{a}_{\mathbf{r}}\left(\mu_{p}^{\mathrm{r}}\right)$ is defined by

$$
\mathbf{a}_{\mathrm{r}}\left(\mu_{p}^{\mathrm{r}}\right)=\left[\begin{array}{lllll}
1 & e^{-j \mu_{p}^{\mathrm{r}}} & e^{-j 2 \mu_{p}^{\mathrm{r}}} & \cdots & e^{-j(N-1) \mu_{p}^{\mathrm{r}}}
\end{array}\right]^{T}
$$

where $\mu_{p}^{\mathrm{r}}=2 \pi \delta_{\mathrm{r}} \sin \theta_{p} . \quad N$ is the number of receive antenna elements, $\delta_{\mathrm{r}}$ is the inter element spacing of the receive array and $\theta_{p}$ is the angle of arrival

\footnotetext{
${ }^{1}$ The same model has been presented in (4.8) and is reproduced here for clarity.
} 
of the $p$ th path. The transmit array response vector is analogously defined by replacing $N$ with $M$ and $\mu_{p}^{\mathrm{r}}$ with $\mu_{p}^{\mathrm{t}}$. A useful representation for our derivations is the vectorized version of the channel matrices defined as

$$
\mathbf{h}(k)=\sum_{p=1}^{P} \alpha_{p}\left(\mathbf{a}_{\mathrm{r}}\left(\mu_{p}^{\mathrm{r}}\right) \otimes \mathbf{a}_{\mathrm{t}}\left(\mu_{p}^{\mathrm{t}}\right)\right) e^{j k \nu_{p}}
$$

We assume that, for the purpose of channel prediction, $K$ samples of the channel are known either from channel estimation or measurement. In practice, the channel estimates contain some amount of error, $\mathbf{w}(k)$ resulting from noise and interference. The estimated channel can therefore be modeled as

$$
\hat{\mathbf{h}}(k)=\mathbf{h}(k)+\mathbf{w}(k) ; \quad z=0,1, \cdots, K-1
$$

For convenience, the $K$ measured samples can be collected into an $N M K \times 1$ vector $\hat{\mathbf{h}}=\left[\begin{array}{lll}\hat{\mathbf{h}}^{T}(0) & \cdots & \hat{\mathbf{h}}^{T}(K-1)\end{array}\right]^{T}$ which can be shown using (8.3) to be

$$
\hat{\mathbf{h}}=\sum_{p=1}^{P} \alpha_{p}\left(\mathbf{a}_{\mathrm{r}}\left(\mu_{p}^{\mathrm{r}}\right) \otimes \mathbf{a}_{\mathrm{t}}\left(\mu_{p}^{\mathrm{t}}\right) \otimes \mathbf{a}_{\mathrm{d}}\left(\nu_{p}\right)\right)+\mathbf{w}
$$

where $\mathbf{a}_{\mathrm{d}}\left(\nu_{p}\right)=\left[\begin{array}{lllll}1 & e^{j \nu_{p}} & e^{j 2 \nu_{p}} & \cdots & e^{j(K-1) \nu_{p}}\end{array}\right]^{T}$. A matrix representation of (8.5) is thus

$$
\begin{aligned}
\hat{\mathbf{h}} & =\left(\mathbf{A}_{\mathrm{r}}\left(\boldsymbol{\mu}^{\mathrm{r}}\right) \diamond \mathbf{A}_{\mathrm{t}}\left(\boldsymbol{\mu}^{\mathrm{t}}\right) \diamond \mathbf{A}_{\mathrm{d}}(\boldsymbol{\nu})\right) \boldsymbol{\alpha}+\mathbf{w} \\
& =\mathbf{A} \boldsymbol{\alpha}+\mathbf{w}
\end{aligned}
$$

where $\boldsymbol{\alpha}=\left[\begin{array}{lll}\alpha_{1} & \cdots & \alpha_{P}\end{array}\right]^{T}$ and $\mathbf{A}_{\mathrm{r}}\left(\boldsymbol{\mu}^{\mathrm{r}}\right)$ is the Vandermonde structured steering matrix defined as

$$
\mathbf{A}_{\mathrm{r}}\left(\boldsymbol{\mu}^{\mathrm{r}}\right)=\left[\begin{array}{lll}
\mathbf{a}_{\mathrm{r}}\left(\mu_{1}^{\mathrm{r}}\right) & \mathbf{a}_{\mathrm{r}}\left(\mu_{2}^{\mathrm{r}}\right) & \cdots \\
\mathbf{a}_{\mathrm{r}}\left(\mu_{P}^{\mathrm{r}}\right)
\end{array}\right]
$$

and $\mathbf{A}_{\mathrm{t}}\left(\boldsymbol{\mu}^{\mathrm{t}}\right)$ and $\mathbf{A}_{\mathrm{d}}(\boldsymbol{\nu})$ are defined analogously.

\section{Prediction Error Bound}

Let the parametrization of the channel be represented by

$$
\Theta=\left[\begin{array}{lllll}
\Re(\boldsymbol{\alpha}) & \mathfrak{I}(\boldsymbol{\alpha}) & \boldsymbol{\mu}^{\mathrm{r}} & \boldsymbol{\mu}^{\mathrm{t}} & \boldsymbol{\nu}
\end{array}\right]
$$


where $\boldsymbol{\mu}^{\mathrm{r}}=\left[\begin{array}{lll}\mu_{1}^{\mathrm{r}} & \cdots & \mu_{P}^{\mathrm{r}}\end{array}\right], \boldsymbol{\mu}^{\mathrm{t}}=\left[\begin{array}{lll}\mu_{1}^{\mathrm{t}} & \cdots & \mu_{P}^{\mathrm{t}}\end{array}\right]$ and $\boldsymbol{\nu}=\left[\begin{array}{lll}\nu_{1} & \cdots & \nu_{P}\end{array}\right]$. $\mathfrak{R}[\cdot]$ and $\mathfrak{I}[\cdot]$ denote the real and imaginary parts of the associated complex number, respectively. To emphasize dependence on channel parameters, (8.6) can now be written as

$$
\hat{\mathbf{h}}=\mathbf{A}(\boldsymbol{\Theta}) \boldsymbol{\alpha}+\mathbf{w}
$$

Assuming that $\mathbf{w} \sim \mathcal{C N}\left(0, \sigma^{2} \mathbf{I}\right)$, the measured data is distributed as $\hat{\mathbf{h}} \sim$ $\mathcal{C N}\left(\boldsymbol{\mu}_{\mathrm{h}}, \sigma^{2} \mathbf{I}\right)$, with $\boldsymbol{\mu}_{\mathrm{h}}=\mathbf{A}(\boldsymbol{\Theta}) \boldsymbol{\alpha}$. Since the channel represents a non-linear function of its parameters, the bound on prediction error can be found using [97]

$$
\operatorname{MSEB}(k)=\operatorname{Tr}\left[\frac{\partial \hat{\mathbf{h}}(k, \boldsymbol{\Theta})}{\partial \boldsymbol{\Theta}} \mathfrak{J}^{-1}(\boldsymbol{\Theta}) \frac{\partial \hat{\mathbf{h}}(k, \boldsymbol{\Theta})}{\partial \boldsymbol{\Theta}}\right]
$$

where $\operatorname{Tr}[\cdot]$ denotes the sum of the diagonal elements of the associated matrix. $\operatorname{MSEB}(k)=\operatorname{Tr}\left[\mathbb{E}\left[(\hat{\mathbf{h}}(k)-\mathbf{h}(k))(\hat{\mathbf{h}}(k)-\mathbf{h}(k))^{H}\right]\right], \mathfrak{J}^{-1}(\boldsymbol{\Theta})$ is the CRLB on multiple parameter estimation, $\mathfrak{J}(\boldsymbol{\Theta})$ is the FIM and the Jacobian in (8.10) is defined as

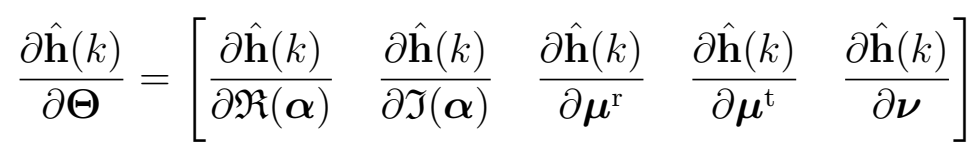

Using Bangs formula [97], entries of the FIM can be evaluated using

$$
[\mathfrak{J}(\boldsymbol{\Theta})]_{i j}=\operatorname{Tr}\left[\mathbf{C}^{-1} \frac{\partial \mathbf{C}}{\partial \boldsymbol{\Theta}_{i}} \mathbf{C}^{-1} \frac{\partial \mathbf{C}}{\partial \boldsymbol{\Theta}_{j}}\right]+2 \Re\left[\frac{\partial \mathbf{h}^{H}}{\partial \boldsymbol{\Theta}_{i}} \mathbf{C}^{-1} \frac{\partial \mathbf{h}}{\partial \boldsymbol{\Theta}_{j}}\right]
$$

where $\mathbf{C}=\sigma^{2} \mathbf{I}$ is the noise covariance matrix. Since the covariance matrix is not dependent on the parameters, only the second term of (8.12) is non-zero and (8.12) reduces to

$$
[\mathfrak{J}(\boldsymbol{\Theta})]=\frac{2}{\sigma^{2}} \mathfrak{R}\left[\frac{\partial \mathbf{h}}{\partial \boldsymbol{\Theta}}^{H} \frac{\partial \mathbf{h}}{\partial \boldsymbol{\Theta}}\right]
$$


The first order derivatives with respect to each of the parameter vector are evaluated as follows

$$
\begin{aligned}
\frac{\partial \mathbf{h}}{\partial \boldsymbol{\mu}^{\mathrm{r}}} & =\left(\mathbf{D}_{\mathrm{r}} \diamond \mathbf{A}_{\mathrm{t}} \diamond \mathbf{A}_{\mathrm{d}}\right) \mathbf{X} \\
\frac{\partial \mathbf{h}}{\partial \boldsymbol{\mu}^{\mathrm{t}}} & =\left(\mathbf{A}_{\mathrm{r}} \diamond \mathbf{D}_{\mathrm{t}} \diamond \mathbf{A}_{\mathrm{d}}\right) \mathbf{X} \\
\frac{\partial \mathbf{h}}{\partial \boldsymbol{\nu}} & =\left(\mathbf{A}_{\mathrm{r}} \diamond \mathbf{A}_{\mathrm{t}} \diamond \mathbf{D}_{\mathrm{d}}\right) \mathbf{X} \\
\frac{\partial \mathbf{h}}{\partial \mathfrak{R}(\boldsymbol{\alpha})} & =\left(\mathbf{A}_{\mathrm{r}} \diamond \mathbf{A}_{\mathrm{t}} \diamond \mathbf{A}_{\mathrm{d}}\right) \\
\frac{\partial \mathbf{h}}{\partial \Im \mathfrak{I}(\boldsymbol{\alpha})} & =j\left(\mathbf{A}_{\mathrm{r}} \diamond \mathbf{A}_{\mathrm{t}} \diamond \mathbf{A}_{\mathrm{d}}\right)
\end{aligned}
$$

where $\mathbf{X}=\operatorname{diag}[\boldsymbol{\alpha}]$ and $\mathbf{D}_{\mathrm{r}}$ is defined for the ULA as

$$
\mathbf{D}_{\mathrm{r}}=\left[\begin{array}{ll}
\frac{\partial \mathbf{a}_{\mathrm{r}}\left(\mu_{1}^{\mathrm{r}}\right)}{\partial \mu_{1}^{\mathrm{r}}} & \cdots \frac{\partial \mathbf{a}_{\mathrm{r}}\left(\mu_{P}^{\mathrm{r}}\right)}{\partial \mu_{P}^{\mathrm{r}}}
\end{array}\right]=-j \mathbf{F}_{N} \mathbf{A}_{\mathrm{r}}
$$

where $\mathbf{F}_{k}$ is a diagonal matrix defined by

$$
\mathbf{F}_{k}=\operatorname{diag}\left[\begin{array}{llll}
0 & 1 & \cdots & k-1
\end{array}\right]
$$

$\mathbf{D}_{\mathrm{t}}$ and $\mathbf{D}_{\mathrm{d}}$ are defined analogously. Using (8.14), the Jacobian in (8.13) can now be expressed as

$$
\frac{\partial \mathbf{h}}{\partial \boldsymbol{\Theta}}=\left[\begin{array}{lll}
\mathbf{A}_{\mathrm{t}} \diamond \mathbf{A}_{\mathrm{d}} \diamond \mathbf{D}_{\mathrm{r}} \mathbf{X} \quad \mathbf{A}_{\mathrm{r}} \diamond \mathbf{D}_{\mathrm{t}} \diamond \mathbf{A}_{\mathrm{d}} \mathbf{X} \mathbf{A}_{\mathrm{r}} \diamond \mathbf{A}_{\mathrm{t}} \diamond \mathbf{D}_{\mathrm{d}} \mathbf{X} \quad \mathbf{A} \quad j \mathbf{A}
\end{array}\right]
$$

which, using the properties of Khatri-Rao and Kronecker products can be expressed as

$$
\frac{\partial \mathbf{h}}{\partial \boldsymbol{\Theta}}=\mathbf{P}_{4} \diamond \mathbf{P}_{3} \diamond \mathbf{P}_{2} \diamond \mathbf{P}_{1}
$$

where

$$
\begin{aligned}
\mathbf{P}_{1} & =\left[\begin{array}{lllll}
\boldsymbol{\alpha}^{T} & \boldsymbol{\alpha}^{T} & \boldsymbol{\alpha}^{T} & \mathbf{1}^{1} & j \mathbf{1}^{T}
\end{array}\right] \\
\mathbf{P}_{2} & =\left[\begin{array}{lllll}
\mathbf{D}_{\mathrm{r}} & \mathbf{A}_{\mathrm{r}} & \mathbf{A}_{\mathrm{r}} & \mathbf{A}_{\mathrm{r}} & \mathbf{A}_{\mathrm{r}}
\end{array}\right] \\
\mathbf{P}_{3} & =\left[\begin{array}{lllll}
\mathbf{A}_{\mathrm{t}} & \mathbf{D}_{\mathrm{t}} & \mathbf{A}_{\mathrm{t}} & \mathbf{A}_{\mathrm{t}} & \mathbf{A}_{\mathrm{t}}
\end{array}\right] \\
\mathbf{P}_{4} & =\left[\begin{array}{lllll}
\mathbf{A}_{\mathrm{d}} & \mathbf{A}_{\mathrm{d}} & \mathbf{D}_{\mathrm{d}} & \mathbf{A}_{\mathrm{d}} & \mathbf{A}_{\mathrm{d}}
\end{array}\right]
\end{aligned}
$$


The FIM is thus

$$
[\mathfrak{J}(\boldsymbol{\Theta})]=\frac{2}{\sigma^{2}} \mathfrak{R}\left[\left(\mathbf{P}_{4} \diamond \mathbf{P}_{3} \diamond \mathbf{P}_{2} \diamond \mathbf{P}_{1}\right)\left(\mathbf{P}_{4} \diamond \mathbf{P}_{3} \diamond \mathbf{P}_{2} \diamond \mathbf{P}_{1}\right)^{H}\right]
$$

Using the following property of Khatri-Rao product

$$
(\mathbf{A} \diamond \mathbf{B})^{H}(\mathbf{A} \diamond \mathbf{B})=\left(\mathbf{A}^{H} \mathbf{A}\right) \odot\left(\mathbf{B}^{H} \mathbf{B}\right)
$$

where $\odot$ denotes element-wise multiplication, (8.23) can be written $a^{2}$

$$
[\mathfrak{J}(\boldsymbol{\Theta})]=\frac{2}{\sigma^{2}} \mathfrak{R}\left[\left(\mathbf{P}_{4}^{H} \mathbf{P}_{4}\right) \odot\left(\mathbf{P}_{3}^{H} \mathbf{P}_{3}\right) \odot\left(\mathbf{P}_{2}^{H} \mathbf{P}_{2}\right) \odot\left(\mathbf{P}_{1}^{H} \mathbf{P}_{1}\right)\right]
$$

Finally, using (8.25) and (8.10), the prediction MSEB can be computed.

\subsubsection{Wideband Channel}

This section present a derivation of the error bound on the prediction of wideband mobile MIMO channel. For clarity, we begin with a brief review of the PRC based model of Section 5.1.

\section{Channel Model}

We consider a wideband ray-based MIMO channel model defined as

$$
\mathbf{H}(t, \tau)=\sum_{p=1}^{P} \alpha_{p} \mathbf{a}_{\mathrm{r}}\left(\mu_{p}^{\mathrm{r}}\right) \mathbf{a}_{\mathrm{t}}^{T}\left(\mu_{p}^{\mathrm{t}}\right) e^{j \omega_{p} t} \delta\left(\tau-\tau_{p}\right)
$$

where $\tau_{p}$ is the delay of the $p$ th path. The frequency response of the channel is obtained via the Fourier transform of (8.26) as

$$
\mathbf{H}(t, f)=\sum_{p=1}^{P} \alpha_{p} \mathbf{a}_{\mathrm{r}}\left(\mu_{p}^{\mathrm{r}}\right) \mathbf{a}_{\mathrm{t}}^{T}\left(\mu_{p}^{\mathrm{t}}\right) e^{j\left(\omega_{p} t-f \tau_{p}\right)}
$$

We assume that no two paths share the same parameter set $\left\{\alpha_{p}, \mu_{p}^{\mathrm{r}}, \mu_{p}^{\mathrm{t}}, \omega_{p}, \tau_{p}\right\}$ but two or more paths may share any subset of the parameter set. Assuming

\footnotetext{
${ }^{2}$ Detailed derivations of the bound are given in Section B.1.
} 
that the system has perfect sample timing and a proper cyclic extension, the sampled frequency response can be expressed as

$$
\mathbf{H}(k, q)=\sum_{p=1}^{P} \alpha_{p} \mathbf{a}_{\mathrm{r}}\left(\mu_{p}^{\mathrm{r}}\right) \mathbf{a}_{\mathrm{t}}^{T}\left(\mu_{p}^{\mathrm{t}}\right) e^{j\left(k \nu_{p}-q \eta_{p}\right)}
$$

where $k$ and $q$ denote the sample and subcarrier index, respectively. $\nu_{p}=$ $T_{\text {samp }} \omega_{p}$ and $\eta_{p}=\Delta f \tau_{p}$ are the normalized Doppler frequency and normalized delay, respectively for symbol period $T_{\text {samp }}$ and subcarrier spacing $\Delta f$. We assume that there are $Q$ equally spaced pilot subcarriers in every OFDM symbol and that $K$ equally spaced pilot symbols are available for the estimation, interpolation and/or prediction. Let $U_{\mathrm{f}}=\left\lceil N_{\mathrm{sc}} / Q\right\rceil$ and $U_{\mathrm{t}}=\left\lceil N_{\text {pilot }} / K\right\rceil$ denote the frequency spacing (measured in number of subcarriers) between adjacent pilot subcarrier and temporal spacing (in number of OFDM symbols) between adjacent pilot symbols, respectively. $N_{\mathrm{sc}}$ is the total number of used subcarriers and $N_{\text {pilot }}$ is the number of OFDM symbols in the training segment. In order to avoid frequency and time domain aliasing, $U_{\mathrm{f}}$ and $U_{\mathrm{t}}$ are chosen such that

$$
U_{\mathrm{f}} \leq \frac{1}{\Delta f \tau_{\max }}
$$

and

$$
U_{\mathrm{t}} \leq \frac{1}{2 \Delta t \omega_{\max }}
$$

where $\tau_{\max }$ and $\omega_{\max }$ are the maximum path delay and Doppler frequency, respectively. We denote the frequency and time indices of the pilots as $\mathcal{F}_{q}=$ $(Q / 2-q) U_{\mathrm{f}} ; \quad q=0,1,2, \cdots, Q-1$ and $\mathcal{T}_{k}=k U_{\mathrm{t}} ; \quad k=0,1,2, \cdots, K-1$, respectively.

\section{Prediction Error Bound}

Using the properties of Kronecker products, the vectorized version of $\mathbf{H}(k, q)$ can be written as

$$
\mathbf{h}(k, q)=\left(\mathbf{A}_{\mathrm{r}}\left(\boldsymbol{\mu}^{\mathrm{r}}\right) \diamond \mathbf{A}_{\mathrm{t}}\left(\boldsymbol{\mu}^{\mathrm{t}}\right)\right) \boldsymbol{\beta}(k, q)
$$


where

$$
\boldsymbol{\beta}(k, q)=\left[\alpha_{1} e^{j\left\{k \nu_{1}-q \eta_{1}\right\}} \cdots \alpha_{p} e^{j\left\{k \nu_{P}-k \eta_{P}\right\}}\right]
$$

and $\mathbf{A}_{\mathrm{r}}\left(\boldsymbol{\mu}^{\mathrm{r}}\right)$ and $\mathbf{A}_{\mathrm{t}}\left(\boldsymbol{\mu}^{\mathrm{r}}\right)$ are the receive and transmit array steering matrices, defined analogously to (8.7) for a ULA. We arrange the $Q K$ known samples into the vector

$$
\begin{aligned}
\mathbf{h}=\left[\mathbf{h}^{T}(1,1), \cdots, \mathbf{h}^{T}(K, 1), \mathbf{h}^{T}(1,2), \cdots,\right. \\
\\
\left.\mathbf{h}^{T}(K, 2), \cdots, \mathbf{h}^{T}(1,3), \cdots, \mathbf{h}^{T}(K, Q)\right]
\end{aligned}
$$

and define Vandermonde matrices $\mathbf{A}_{\mathrm{d}}$ and $\mathbf{A}_{\mathrm{f}}$ as

$$
\mathbf{A}_{\mathrm{d}}=\left[\begin{array}{ccc}
1 & \cdots & 1 \\
e^{j \nu_{1}} & \cdots & e^{j \nu_{P}} \\
\vdots & \ddots & \vdots \\
e^{j(K-1) \nu_{1}} & \cdots & e^{j(K-1) \nu_{P}}
\end{array}\right]
$$

and

It can easily be verified that

$$
\mathbf{A}_{\mathrm{f}}=\left[\begin{array}{ccc}
1 & \cdots & 1 \\
e^{-j \eta_{1}} & \cdots & e^{-j \eta_{P}} \\
\vdots & \ddots & \vdots \\
e^{-j(Q-1) \eta_{1}} & \cdots & e^{-j(Q-1) \eta_{P}}
\end{array}\right]
$$

$$
\begin{aligned}
\mathbf{h} & =\left(\mathbf{A}_{\mathrm{r}} \diamond \mathbf{A}_{\mathrm{t}} \diamond \mathbf{A}_{\mathrm{d}} \diamond \mathbf{A}_{\mathrm{f}}\right) \boldsymbol{\beta} \\
& =\mathbf{A} \boldsymbol{\beta}
\end{aligned}
$$

where $\mathbf{A}=\left(\mathbf{A}_{\mathrm{r}} \diamond \mathbf{A}_{\mathrm{t}} \diamond \mathbf{A}_{\mathrm{d}} \diamond \mathbf{A}_{\mathrm{f}}\right)$. Let the parameterization of the wideband channel be denoted by the $(6 P+1) \times 1$ vector

$$
\boldsymbol{\Theta}=\left[\sigma^{2},\left(\boldsymbol{\mu}^{\mathrm{r}}\right)^{T},\left(\boldsymbol{\mu}^{\mathrm{t}}\right)^{T}, \boldsymbol{\gamma}^{T}, \boldsymbol{\eta}^{T}, \mathfrak{R}\left(\boldsymbol{\beta}^{T}\right), \mathfrak{I}\left(\boldsymbol{\beta}^{T}\right)\right]^{T}
$$

As in the narrowband case, the prediction error covariance can be bounded by [97]

$$
\begin{aligned}
\mathbf{C}_{e}(k, q) & =\mathbb{E}\left[(\hat{\mathbf{h}}(k, q)-\mathbf{h}(k, q))(\hat{\mathbf{h}}(k, q)-\mathbf{h}(k, q))^{H}\right] \\
& \geq \frac{\partial \mathbf{h}(k, q)}{\partial \boldsymbol{\Theta}} \mathfrak{J}^{-1}(\boldsymbol{\Theta}) \frac{\partial \mathbf{h}(k, q)}{\partial \boldsymbol{\Theta}}
\end{aligned}
$$


where $\mathfrak{J}^{-1}(\boldsymbol{\Theta})$ is the lower bound on the estimation of channel parameters, $\mathfrak{J}(\boldsymbol{\Theta})$ is the FIM, which can be computed element-wise using (8.5). After evaluating the derivatives and performing straightforward but tedious simplifications (see Appendix B.3), $\mathfrak{J}(\boldsymbol{\Theta})$ is obtained as

$$
\mathfrak{J}(\boldsymbol{\Theta})=\left[\begin{array}{cc}
\frac{K Q N M}{\sigma^{4}} & \mathbf{0} \\
\mathbf{0}^{T} & \mathfrak{K}(\boldsymbol{\Theta})
\end{array}\right]
$$

with the Fisher information submatrix defined as

$$
[\mathfrak{K}(\Theta)]=\frac{2}{\sigma^{2}} \mathfrak{R}\left[\left(\mathbf{P}_{5}^{H} \mathbf{P}_{5}\right) \odot\left(\mathbf{P}_{4}^{H} \mathbf{P}_{4}\right) \odot\left(\mathbf{P}_{3}^{H} \mathbf{P}_{3}\right) \odot\left(\mathbf{P}_{2}^{H} \mathbf{P}_{2}\right) \odot\left(\mathbf{P}_{1}^{H} \mathbf{P}_{1}\right)\right]
$$

where $\mathbf{P}_{1}-\mathbf{P}_{5}$ are defined as

$$
\begin{aligned}
\mathbf{P}_{1} & =\left[\begin{array}{llllll}
\boldsymbol{\alpha}^{T} & \boldsymbol{\alpha}^{T} & \boldsymbol{\alpha}^{T} & \boldsymbol{\alpha}^{T} & \mathbf{1}^{1} & j \mathbf{1}^{T}
\end{array}\right] \\
\mathbf{P}_{2} & =\left[\begin{array}{llllll}
\mathbf{D}_{\mathrm{r}} & \mathbf{A}_{\mathrm{r}} & \mathbf{A}_{\mathrm{r}} & \mathbf{A}_{\mathrm{r}} & \mathbf{A}_{\mathrm{r}} & \mathbf{A}_{\mathrm{r}}
\end{array}\right] \\
\mathbf{P}_{3} & =\left[\begin{array}{llllll}
\mathbf{A}_{\mathrm{t}} & \mathbf{D}_{\mathrm{t}} & \mathbf{A}_{\mathrm{t}} & \mathbf{A}_{\mathrm{t}} & \mathbf{A}_{\mathrm{t}} & \mathbf{A}_{\mathrm{t}}
\end{array}\right] \\
\mathbf{P}_{4} & =\left[\begin{array}{llllll}
\mathbf{A}_{\mathrm{d}} & \mathbf{A}_{\mathrm{d}} & \mathbf{D}_{\mathrm{d}} & \mathbf{A}_{\mathrm{d}} & \mathbf{A}_{\mathrm{d}} & \mathbf{A}_{\mathrm{d}}
\end{array}\right] \\
\mathbf{P}_{5} & =\left[\begin{array}{llllll}
\mathbf{A}_{\mathrm{f}} & \mathbf{A}_{\mathrm{f}} & \mathbf{A}_{\mathrm{f}} & \mathbf{D}_{\mathrm{f}} & \mathbf{A}_{\mathrm{f}} & \mathbf{A}_{\mathrm{f}}
\end{array}\right]
\end{aligned}
$$

The matrices $\mathbf{D}_{\mathrm{r}}, \mathbf{D}_{\mathrm{t}}, \mathbf{D}_{\mathrm{d}}$ and $\mathbf{D}_{\mathrm{f}}$ are defined analogous to (8.15). Detail of the mathematical derivations can be found in Appendix B.3.

\subsubsection{D MIMO Channel Prediction Error Bound}

In this Section, we derive expression for the bound on the prediction of 3D channels. As in Section 4.5, we consider a narrowband system with a UPA at the BS and a ULA at the MS. Consider the vectorized channel in (4.117) which can be expressed in matrix form $\mathrm{as}^{3}$

$$
\mathbf{h}(k)=\left(\mathbf{A}_{\mathrm{r}} \diamond \mathbf{A}_{\mathrm{tx}} \diamond \mathbf{A}_{\mathrm{ty}} \diamond \mathbf{f}_{\mathrm{d}}\right) \boldsymbol{\alpha}
$$

\footnotetext{
${ }^{3}$ We omit the noise term here for simplicity.
} 
where $\mathbf{f}(k)=\left[e^{j k \nu_{1}}, e^{j k \nu_{2}}, \cdots, e^{j k \nu_{P}}\right]$. Collecting the $K$ observation vector into $\mathbf{h}=\left[\hat{\mathbf{h}}^{T}(0), \hat{\mathbf{h}}^{T}(1), \cdots, \hat{\mathbf{h}}^{T}(K-1)\right]^{T}$ yields

$$
\begin{aligned}
\mathbf{h} & =\left(\mathbf{A}_{\mathrm{r}} \diamond \mathbf{A}_{\mathrm{tx}} \diamond \mathbf{A}_{\mathrm{ty}} \diamond \mathbf{A}_{\mathrm{d}}\right) \boldsymbol{\alpha} \\
& =\mathbf{A} \boldsymbol{\alpha}
\end{aligned}
$$

Denoting the channel parametrization as

$$
\boldsymbol{\Theta}=\left[\Re\left(\boldsymbol{\alpha}^{T}\right), \mathfrak{I}\left(\boldsymbol{\alpha}^{T}\right), \boldsymbol{\mu}_{\mathrm{r}}^{T}, \boldsymbol{\mu}_{\mathrm{tx}}^{T}, \boldsymbol{\mu}_{\mathrm{ty}}^{T}, \boldsymbol{\nu}^{T}\right]
$$

As in (8.38), the covariance of the 3D channel parameter estimates is bounded by

$$
\mathrm{C}_{\Theta \Theta} \geq \mathfrak{J}^{-1}
$$

where

$$
\mathfrak{J}=-\mathbb{E}\left[\frac{\partial p(\mathbf{h} ; \boldsymbol{\Theta})}{\partial \boldsymbol{\Theta}} \frac{\partial p(\mathbf{h} ; \boldsymbol{\Theta})^{H}}{\partial \boldsymbol{\Theta}}\right]
$$

which can be computed using (8.5). In order to derive an expression for the FIM using (8.10), we need to evaluate the Jacobian

$$
\frac{\partial \mathbf{h}}{\partial \boldsymbol{\Theta}}=\left[\begin{array}{llllll}
\frac{\partial \mathbf{h}}{\partial \mathfrak{R}(\boldsymbol{\alpha})} & \frac{\partial \mathfrak{h}}{\partial \mathfrak{I}(\boldsymbol{\alpha})} & \frac{\partial \mathfrak{h}}{\partial \boldsymbol{\mu}_{\mathrm{r}}} & \frac{\partial \mathfrak{h}}{\partial \boldsymbol{\mu}_{\mathrm{tx}}} & \frac{\partial \mathfrak{h}}{\partial \boldsymbol{\mu}_{\mathrm{ty}}} & \frac{\partial \mathfrak{h}}{\partial \boldsymbol{\nu}}
\end{array}\right]
$$

Using (8.47) and performing some mathematical simplifications, (8.51) becomes

$$
\begin{aligned}
\frac{\partial \mathbf{h}}{\partial \boldsymbol{\Theta}}= & {\left[\begin{array}{lll}
\mathbf{A} & j \mathbf{A} & \left(\mathbf{D}_{\mathrm{r}} \diamond \mathbf{A}_{\mathrm{tx}} \diamond \mathbf{A}_{\mathrm{ty}} \diamond \mathbf{A}_{\mathrm{d}}\right) \mathbf{Y} \quad\left(\mathbf{A}_{\mathrm{r}} \diamond \mathbf{D}_{\mathrm{tx}} \diamond \mathbf{A}_{\mathrm{ty}} \diamond \mathbf{A}_{\mathrm{d}}\right) \mathbf{Y} \\
& \left(\mathbf{A}_{\mathrm{r}} \diamond \mathbf{A}_{\mathrm{tx}} \diamond \mathbf{D}_{\mathrm{ty}} \diamond \mathbf{A}_{\mathrm{d}}\right) \mathbf{Y} \quad\left(\mathbf{A}_{\mathrm{r}} \diamond \mathbf{A}_{\mathrm{tx}} \diamond \mathbf{A}_{\mathrm{ty}} \diamond \mathbf{D}_{\mathrm{d}}\right) \mathbf{Y}
\end{array}\right] }
\end{aligned}
$$

where $\mathbf{Y}$ is the diagonal matrix

$$
\mathbf{Y}=\left[\begin{array}{cccc}
\alpha_{1} & 0 & \cdots & 0 \\
0 & \alpha_{2} & \cdots & 0 \\
\vdots & \vdots & \ddots & \vdots \\
0 & 0 & \cdots & \alpha_{P}
\end{array}\right]
$$


and $\mathbf{D}_{\mathrm{r}}$ is defined as

$$
\begin{aligned}
\mathbf{D}_{\mathrm{r}} & =\left[\begin{array}{lll}
\frac{\partial\left[\mathbf{A}_{\mathrm{r}}\right]_{:, 1}}{\partial \mu_{1}^{\mathrm{r}}} & \frac{\partial\left[\mathbf{A}_{\mathrm{r}}\right]_{:, 2}}{\partial \mu_{2}^{\mathrm{r}}} & \cdots \\
= & \cdots \frac{\partial\left[\mathbf{A}_{\mathrm{r}}\right]_{:, P}}{\partial \mu_{P}^{\mathrm{r}}}
\end{array}\right] \\
& =\left[\begin{array}{ccc}
0 & \cdots & 0 \\
j e^{j \mu_{1}^{\mathrm{r}}} & \cdots & j e^{j \mu_{P}^{\mathrm{r}}} \\
\vdots & \ddots & \vdots \\
j e^{j(M-1) \mu_{1}^{\mathrm{r}}} & \cdots & j e^{j(M-1) \mu_{P}^{\mathrm{r}}}
\end{array}\right]
\end{aligned}
$$

where $[\mathbf{A}]_{:, n}$ denotes the nth column of $\mathbf{A}$. Matrices $\mathbf{D}_{\mathrm{tx}}, \mathbf{D}_{\mathrm{ty}}$, and $\mathbf{D}_{\mathrm{d}}$ are defined analogously. Denoting

$$
\begin{aligned}
\mathbf{P}_{1} & =\left[\begin{array}{llllll}
\mathbf{1}^{T} & \mathbf{1}^{T} & \boldsymbol{\alpha}^{T} & \boldsymbol{\alpha}^{T} & \boldsymbol{\alpha}^{T} & \boldsymbol{\alpha}^{T}
\end{array}\right] \\
\mathbf{P}_{2} & =\left[\begin{array}{lllllll}
\mathbf{A}_{\mathrm{r}} & \mathbf{A}_{\mathrm{r}} & \mathbf{D}_{\mathrm{r}} & \mathbf{A}_{\mathrm{r}} & \mathbf{A}_{\mathrm{r}} & \mathbf{A}_{\mathrm{r}}
\end{array}\right] \\
\mathbf{P}_{3} & =\left[\begin{array}{lllllll}
\mathbf{A}_{\mathrm{tx}} & \mathbf{A}_{\mathrm{tx}} & \mathbf{A}_{\mathrm{tx}} & \mathbf{D}_{\mathrm{tx}} & \mathbf{A}_{\mathrm{tx}} & \mathbf{A}_{\mathrm{tx}}
\end{array}\right] \\
\mathbf{P}_{4} & =\left[\begin{array}{llllll}
\mathbf{A}_{\mathrm{ty}} & \mathbf{A}_{\mathrm{ty}} & \mathbf{A}_{\mathrm{ty}} & \mathbf{A}_{\mathrm{ty}} & \mathbf{D}_{\mathrm{ty}} & \mathbf{A}_{\mathrm{ty}}
\end{array}\right] \\
\mathbf{P}_{5} & =\left[\begin{array}{llllll}
\mathbf{A}_{\mathrm{d}} & \mathbf{A}_{\mathrm{d}} & \mathbf{A}_{\mathrm{d}} & \mathbf{A}_{\mathrm{d}} & \mathbf{A}_{\mathrm{d}} & \mathbf{D}_{\mathrm{d}}
\end{array}\right]
\end{aligned}
$$

(8.51) can be shown to be

$$
\frac{\partial \mathbf{h}}{\partial \boldsymbol{\Theta}}=\mathbf{P}_{1} \diamond \mathbf{P}_{2} \diamond \mathbf{P}_{3} \diamond \mathbf{P}_{4} \diamond \mathbf{P}_{5}
$$

Substituting (8.56) into (8.50) and using the properties of Kronecker products yields

$$
\begin{aligned}
\mathfrak{J} & =\frac{2}{\sigma^{2}} \mathfrak{R}\left[\left(\mathbf{P}_{1} \diamond \mathbf{P}_{2} \diamond \mathbf{P}_{3} \diamond \mathbf{P}_{4} \diamond \mathbf{P}_{5}\right)^{H}\left(\mathbf{P}_{1} \diamond \mathbf{P}_{2} \diamond \mathbf{P}_{3} \diamond \mathbf{P}_{4} \diamond \mathbf{P}_{5}\right)\right] \\
& =\frac{2}{\sigma^{2}} \mathfrak{R}\left[\left(\mathbf{P}_{1}^{H} \mathbf{P}_{1}\right) \odot\left(\mathbf{P}_{2}^{H} \mathbf{P}_{2}\right) \odot\left(\mathbf{P}_{3}^{H} \mathbf{P}_{3}\right) \odot\left(\mathbf{P}_{4}^{H} \mathbf{P}_{4}\right) \odot\left(\mathbf{P}_{5}^{H} \mathbf{P}_{5}\right)\right]
\end{aligned}
$$

Putting (8.57) into (8.49) gives the lower bound on the variance of the parameter estimates, and the prediction error bound can be computed using

$$
\mathbb{E}(k) \geq \frac{\partial \mathbf{h}^{H}(k)}{\partial \boldsymbol{\Theta}} \mathfrak{J}^{-1} \frac{\partial \mathbf{h}(k)}{\partial \boldsymbol{\Theta}}
$$

where

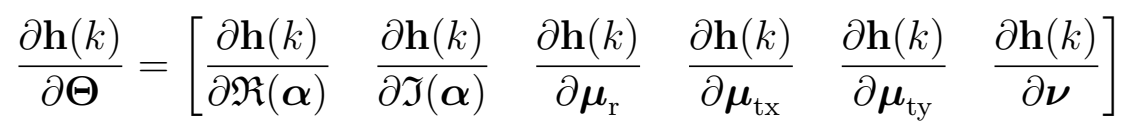




\subsection{Polarized MIMO Channel Prediction Error Bound}

In this section, we derive the performance bound for estimation and prediction of narrowband MIMO channel with polarized antenna array. Consider the $N_{\text {pol }} N \times N_{\text {pol }} M$ polarized MIMO channel in (7.4)

$$
\mathbf{H}(k)=\sum_{p=1}^{P} \mathbf{S}_{\mathrm{r}}\left(\theta_{p}\right)\left[\begin{array}{ll}
\alpha_{h h, p} & \alpha_{h v, p} \\
\alpha_{v h, p} & \alpha_{v v, p}
\end{array}\right] \mathbf{S}_{\mathrm{t}}^{T}\left(\phi_{p}\right) e^{j k \nu_{p}}
$$

where $N_{\text {pol }}$ denotes the number of polarization dimensions. We consider the antenna configurations in Fig. 7.1 such that $N_{\text {pol }}=2$. The vectorized data obtained by stacking the columns of $\mathbf{H}(k)$ is thus

$$
\mathbf{h}(k)=\left[\begin{array}{llll}
\left(\mathbf{S}_{\mathrm{rh}} \diamond \mathbf{S}_{\mathrm{th}}\right) & \left(\mathbf{S}_{\mathrm{rv}} \diamond \mathbf{S}_{\mathrm{th}}\right) \quad\left(\mathbf{S}_{\mathrm{rh}} \diamond \mathbf{S}_{\mathrm{tv}}\right) \quad\left(\mathbf{S}_{\mathrm{rv}} \diamond \mathbf{S}_{\mathrm{tv}}\right)
\end{array}\right]\left[\begin{array}{c}
\boldsymbol{\beta}_{\mathrm{hh}} \\
\boldsymbol{\beta}_{\mathrm{vh}} \\
\boldsymbol{\beta}_{\mathrm{hv}} \\
\boldsymbol{\beta}_{\mathrm{vv}}
\end{array}\right]
$$

where $\boldsymbol{\beta}_{\mathrm{hh}}$ is defined as

$$
\boldsymbol{\beta}_{\mathbf{h h}}=\left[\alpha_{\mathrm{hh}, 1} \cdots \alpha_{\mathrm{hh}, P}\right] \odot\left[e^{j k \nu_{1}} \cdots e^{j k \nu_{P}}\right]
$$

Assuming that the $K$ samples are collected into a vector $\mathbf{h}=\left[\begin{array}{lll}\mathbf{h}(1)^{T} & \cdots & \mathbf{h}^{T}(K)\end{array}\right] \in \mathbb{C}^{2 N_{\text {pol }} N M K \times 1}$ and using (8.61), we obtained an expression for $\mathbf{h}$ as

$$
\begin{aligned}
\mathbf{h}=[ & \left(\mathbf{S}_{\mathrm{rh}} \diamond \mathbf{S}_{\mathrm{th}} \diamond \mathbf{A}_{\mathrm{d}}\right) \quad\left(\mathbf{S}_{\mathrm{rv}} \diamond \mathbf{S}_{\mathrm{th}} \diamond \mathbf{A}_{\mathrm{d}}\right) \quad\left(\mathbf{S}_{\mathrm{rh}} \diamond \mathbf{S}_{\mathrm{tv}} \diamond \mathbf{A}_{\mathrm{d}}\right) \\
& \left.\left(\mathbf{S}_{\mathrm{rv}} \diamond \mathbf{S}_{\mathrm{tv}} \diamond \mathbf{A}_{\mathrm{d}}\right)\right] \boldsymbol{\alpha}
\end{aligned}
$$

where $\boldsymbol{\alpha}=\left[\begin{array}{llll}\boldsymbol{\alpha}_{\mathrm{hh}}^{T} & \boldsymbol{\alpha}_{\mathrm{vh}}^{T} & \boldsymbol{\alpha}_{\mathrm{hv}}^{T} & \boldsymbol{\alpha}_{\mathrm{vv}}^{T}\end{array}\right]^{T}$ and $\mathbf{A}_{\mathrm{d}}$ is the $K \times P$ Vandermonde structured matrix in (8.34). Note that (8.63) can be expressed as a summation over the $2 N_{\text {pol }}$ polarization dimensions:

$$
\begin{aligned}
\mathbf{h} & =\left(\mathbf{S}_{\mathrm{rh}} \diamond \mathbf{S}_{\mathrm{th}} \diamond \mathbf{A}_{\mathrm{d}}\right) \boldsymbol{\alpha}_{\mathrm{hh}}+\left(\mathbf{S}_{\mathrm{rv}} \diamond \mathbf{S}_{\mathrm{th}} \diamond \mathbf{A}_{\mathrm{d}}\right) \boldsymbol{\alpha}_{\mathrm{hv}} \\
& +\left(\mathbf{S}_{\mathrm{rh}} \diamond \mathbf{S}_{\mathrm{tv}} \diamond \mathbf{A}_{\mathrm{d}}\right) \boldsymbol{\alpha}_{\mathrm{vh}}+\left(\mathbf{S}_{\mathrm{rv}} \diamond \mathbf{S}_{\mathrm{tv}} \diamond \mathbf{A}_{\mathrm{d}}\right) \boldsymbol{\alpha}_{\mathrm{vv}}
\end{aligned}
$$


Let the parametrization of the polarized MIMO channel be

$$
\begin{gathered}
\boldsymbol{\Theta}=\left[\boldsymbol{\theta}^{T}, \boldsymbol{\phi}^{T}, \boldsymbol{\nu}^{T}, \mathfrak{R}\left(\boldsymbol{\alpha}_{h h}^{T}\right), \mathfrak{R}\left(\boldsymbol{\alpha}_{v h}^{T}\right), \mathfrak{R}\left(\boldsymbol{\alpha}_{h v}^{T}\right), \mathfrak{R}\left(\boldsymbol{\alpha}_{v v}^{T}\right),\right. \\
\left.\mathfrak{I}\left(\boldsymbol{\alpha}_{h h}^{T}\right), \mathfrak{I}\left(\boldsymbol{\alpha}_{v h}^{T}\right), \mathfrak{I}\left(\boldsymbol{\alpha}_{h v}^{T}\right), \mathfrak{I}\left(\boldsymbol{\alpha}_{v v}^{T}\right)\right]^{T}
\end{gathered}
$$

As in the previous sections. the covariance of the parameter estimates and estimation/prediction error at the $k$ th instant are bounded by (8.50) and (8.58), respectively. Let the derivatives of the polarized steering matrices be denoted by

$$
\begin{aligned}
& \mathbf{D}_{\mathrm{rh}}=\left[\begin{array}{lll}
\frac{\partial \mathbf{s}_{\mathrm{rh}}\left(\theta_{1}\right)}{\partial \theta_{1}} & \cdots & \frac{\partial \mathbf{s}_{\mathrm{rh}}\left(\theta_{P}\right)}{\partial \theta_{P}}
\end{array}\right] \\
& \mathbf{D}_{\mathrm{rv}}=\left[\begin{array}{lll}
\frac{\partial \mathbf{s}_{\mathrm{rv}}\left(\theta_{1}\right)}{\partial \theta_{1}} & \cdots & \frac{\partial \mathbf{s}_{\mathrm{rv}}\left(\theta_{P}\right)}{\partial \theta_{P}}
\end{array}\right] \\
& \mathbf{D}_{\mathrm{th}}=\left[\begin{array}{lll}
\frac{\partial \mathbf{s}_{\mathrm{th}}\left(\phi_{1}\right)}{\partial \phi_{1}} & \cdots & \frac{\partial \mathbf{s}_{\mathrm{th}}\left(\phi_{P}\right)}{\partial \phi_{P}}
\end{array}\right] \\
& \mathbf{D}_{\mathrm{tv}}=\left[\begin{array}{lll}
\frac{\partial \mathbf{s}_{\mathrm{tv}}\left(\phi_{1}\right)}{\partial \phi_{1}} & \cdots & \frac{\partial \mathbf{s}_{\mathrm{tv}}\left(\phi_{P}\right)}{\partial \phi_{P}}
\end{array}\right]
\end{aligned}
$$

and a vector containing the derivatives of the columns of $\mathbf{A}_{\mathrm{d}}$ as

$$
\mathbf{D}_{\mathrm{d}}=\left[\begin{array}{lll}
\frac{\partial \mathbf{a}_{\mathrm{d}}\left(\nu_{1}\right)}{\partial \nu_{1}} & \cdots & \frac{\partial \mathbf{a}_{\mathrm{d}}\left(\nu_{P}\right)}{\partial \nu_{P}}
\end{array}\right]
$$

After some simplifications, the derivatives of (8.64) with respect to the channel parameters can be shown to be

$$
\begin{aligned}
\frac{\partial \mathbf{h}}{\partial \boldsymbol{\theta}} & =\left(\mathbf{D}_{\mathrm{rh}} \diamond \mathbf{S}_{\mathrm{th}} \diamond \mathbf{A}_{\mathrm{d}}\right) \mathbf{X}_{\mathrm{hh}}+\left(\mathbf{D}_{\mathrm{rv}} \diamond \mathbf{S}_{\mathrm{th}} \diamond \mathbf{A}_{\mathrm{d}}\right) \mathbf{X}_{\mathrm{vh}} \\
& +\left(\mathbf{D}_{\mathrm{rh}} \diamond \mathbf{S}_{\mathrm{tv}} \diamond \mathbf{A}_{\mathrm{d}}\right) \mathbf{X}_{\mathrm{hv}}+\left(\mathbf{D}_{\mathrm{rv}} \diamond \mathbf{S}_{\mathrm{tv}} \diamond \mathbf{A}_{\mathrm{d}}\right) \mathbf{X}_{\mathrm{vv}} \\
\frac{\partial \mathbf{h}}{\partial \boldsymbol{\phi}} & =\left(\mathbf{S}_{\mathrm{rh}} \diamond \mathbf{D}_{\mathrm{th}} \diamond \mathbf{A}_{\mathrm{d}}\right) \mathbf{X}_{\mathrm{hh}}+\left(\mathbf{S}_{\mathrm{rv}} \diamond \mathbf{D}_{\mathrm{th}} \diamond \mathbf{A}_{\mathrm{d}}\right) \mathbf{X}_{\mathrm{vh}} \\
& +\left(\mathbf{S}_{\mathrm{rh}} \diamond \mathbf{D}_{\mathrm{tv}} \diamond \mathbf{A}_{\mathrm{d}}\right) \mathbf{X}_{\mathrm{hv}}+\left(\mathbf{S}_{\mathrm{rv}} \diamond \mathbf{D}_{\mathrm{tv}} \diamond \mathbf{A}_{\mathrm{d}}\right) \mathbf{X}_{\mathrm{vv}} \\
\frac{\partial \mathbf{h}}{\partial \boldsymbol{\nu}} & =\left(\mathbf{S}_{\mathrm{rh}} \diamond \mathbf{S}_{\mathrm{th}} \diamond \mathbf{D}_{\mathrm{d}}\right) \mathbf{X}_{\mathrm{hh}}+\left(\mathbf{S}_{\mathrm{rv}} \diamond \mathbf{S}_{\mathrm{th}} \diamond \mathbf{D}_{\mathrm{d}}\right) \mathbf{X}_{\mathrm{vh}} \\
& +\left(\mathbf{S}_{\mathrm{rh}} \diamond \mathbf{S}_{\mathrm{tv}} \diamond \mathbf{D}_{\mathrm{d}}\right) \mathbf{X}_{\mathrm{hv}}+\left(\mathbf{S}_{\mathrm{rv}} \diamond \mathbf{S}_{\mathrm{tv}} \diamond \mathbf{D}_{\mathrm{d}}\right) \mathbf{X}_{\mathrm{vv}}
\end{aligned}
$$


where

$$
\mathbf{X}_{\mathrm{hh}}=\operatorname{diag}\left[\boldsymbol{\alpha}_{\mathrm{hh}}\right]
$$

$\mathbf{X}_{\mathrm{vh}}, \mathbf{X}_{\mathrm{hv}}$ and $\mathbf{X}_{\mathrm{hh}}$ are defined analogously. Similarly, the derivatives with respect to the polarimetric weights are obtained as

$$
\begin{aligned}
& \frac{\partial \mathbf{h}}{\partial \mathfrak{R}\left(\boldsymbol{\alpha}_{\mathrm{hh}}^{T}\right)}=\left(\mathbf{S}_{\mathrm{rh}} \diamond \mathbf{S}_{\mathrm{th}} \diamond \mathbf{A}_{\mathrm{d}}\right) \\
& \frac{\partial \mathbf{h}}{\partial \mathfrak{R}\left(\boldsymbol{\alpha}_{\mathrm{vh}}^{T}\right)}=\left(\mathbf{S}_{\mathrm{rv}} \diamond \mathbf{S}_{\mathrm{th}} \diamond \mathbf{A}_{\mathrm{d}}\right) \\
& \frac{\partial \mathbf{h}}{\partial \mathfrak{R}\left(\boldsymbol{\alpha}_{\mathrm{hv}}^{T}\right)}=\left(\mathbf{S}_{\mathrm{rh}} \diamond \mathbf{S}_{\mathrm{tv}} \diamond \mathbf{A}_{\mathrm{d}}\right) \\
& \frac{\partial \mathbf{h}}{\partial \mathfrak{R}\left(\boldsymbol{\alpha}_{\mathrm{vv}}^{T}\right)}=\left(\mathbf{S}_{\mathrm{rv}} \diamond \mathbf{S}_{\mathrm{tv}} \diamond \mathbf{A}_{\mathrm{d}}\right) \\
& \frac{\partial \mathbf{h}}{\partial \mathfrak{I}\left(\boldsymbol{\alpha}_{\mathrm{hh}}^{T}\right)}=j\left(\mathbf{S}_{\mathrm{rh}} \diamond \mathbf{S}_{\mathrm{th}} \diamond \mathbf{A}_{\mathrm{d}}\right) \\
& \frac{\partial \mathbf{h}}{\partial \mathfrak{I}\left(\boldsymbol{\alpha}_{\mathrm{vh}}^{T}\right)}=j\left(\mathbf{S}_{\mathrm{rv}} \diamond \mathbf{S}_{\mathrm{th}} \diamond \mathbf{A}_{\mathrm{d}}\right) \\
& \frac{\partial \mathbf{h}}{\partial \mathfrak{I}\left(\boldsymbol{\alpha}_{\mathrm{hv}}^{T}\right)}=j\left(\mathbf{S}_{\mathrm{rh}} \diamond \mathbf{S}_{\mathrm{tv}} \diamond \mathbf{A}_{\mathrm{d}}\right) \\
& \frac{\partial \mathbf{h}}{\partial \mathfrak{I}\left(\boldsymbol{\alpha}_{\mathrm{vv}}^{T}\right)}=j\left(\mathbf{S}_{\mathrm{rv}} \diamond \mathbf{S}_{\mathrm{tv}} \diamond \mathbf{A}_{\mathrm{d}}\right)
\end{aligned}
$$

Using equations (8.71)-(8.82) the Jacobian $\frac{\partial \mathbf{h}}{\partial \boldsymbol{\Theta}}$ can be computed using

$$
\begin{aligned}
\mathbf{P}= & {\left[\begin{array}{llllll}
\frac{\partial \mathbf{h}}{\partial \boldsymbol{\theta}} & \frac{\partial \mathbf{h}}{\partial \boldsymbol{\phi}} & \frac{\partial \mathbf{h}}{\partial \boldsymbol{\nu}} & \frac{\partial \mathbf{h}}{\partial \mathfrak{R}\left(\boldsymbol{\alpha}_{h h}^{T}\right)} & \frac{\partial \mathbf{h}}{\partial \mathfrak{R}\left(\boldsymbol{\alpha}_{v h}^{T}\right)} & \frac{\partial \mathbf{h}}{\partial \mathfrak{R}\left(\boldsymbol{\alpha}_{h v}^{T}\right)} \\
& \frac{\partial \mathbf{h}}{\partial \mathfrak{R}\left(\boldsymbol{\alpha}_{v v}^{T}\right)} & \frac{\partial \mathbf{h}}{\partial \mathfrak{I}\left(\boldsymbol{\alpha}_{h h}^{T}\right)} & \frac{\partial \mathbf{h}}{\partial \mathfrak{I}\left(\boldsymbol{\alpha}_{v h}^{T}\right)} & \frac{\partial \mathbf{h}}{\partial \mathfrak{I}\left(\boldsymbol{\alpha}_{h v}^{T}\right)} & \frac{\partial \mathbf{h}}{\partial \mathfrak{I}\left(\boldsymbol{\alpha}_{v v}^{T}\right)}
\end{array}\right] }
\end{aligned}
$$

The Fisher information sub-matrix is then obtained as ${ }^{4}$

$$
\mathfrak{K}(\boldsymbol{\Theta})=\frac{2}{\sigma^{2}}\left[\mathbf{P}^{H} \mathbf{P}\right]
$$

Since the noise variance is typically unknown in practice and needs to be estimated, we reparametrized the channel to include the noise variance and

\footnotetext{
${ }^{4}$ Assuming that the noise variance is known, $\mathfrak{J}(\boldsymbol{\Theta})$ corresponds to the FIM.
} 
the FIM is thus obtained as

$$
\mathfrak{J}(\boldsymbol{\Theta})=\left[\begin{array}{cc}
\frac{2 N_{p o l} N M K}{\sigma^{2}} & \mathbf{0} \\
\mathbf{0}^{T} & \mathfrak{K}(\boldsymbol{\Theta})
\end{array}\right]
$$

It is straightforward to derive expressions for wideband polarized MIMO channels following a similar procedure.

\subsection{Asymptotic Error Bound}

As shown in Section 8.1, the bound on the prediction error can be found using (8.10). However, this is not readily interpretable and its dependence on the actual channel parameters necessitates numerical averaging for specified probability distributions. The computational load becomes significant for large values of $N, M, K$ and/or $Q$. In this section, we derive simple and easily interpretable closed-from expressions for the lower bound on the prediction MSE and NMSE.

\subsubsection{Narrowband Channels}

Using (8.1), entries of the MIMO channel matrix can be expressed as

$$
h(n, m, k)=\sum_{p=1}^{P} \alpha_{p} e^{j\left(k \nu_{p}-(n-1) \mu_{p}^{\mathrm{r}}-(m-1) \mu_{p}^{\mathrm{t}}\right)}
$$

for all $n=1, \cdots, N, m=1, \cdots, M$ and $k=0, \cdots, K-1$. For convenience, the channel is re-parametrized as

$$
\boldsymbol{\Theta}=\left[\boldsymbol{\theta}_{1}^{T}, \cdots, \boldsymbol{\theta}_{P}^{T}\right]
$$

where

$$
\boldsymbol{\theta}_{p}=\left[\begin{array}{lllll}
\Re\left(\alpha_{p}\right) & \mathfrak{I}\left(\alpha_{p}\right) & \mu_{p}^{\mathrm{r}} & \mu_{p}^{\mathrm{t}} & \nu_{p}
\end{array}\right]^{T}
$$

Assuming that the error is Gaussian with variance $\sigma^{2}$, entries of the FIM can be computed using

$$
[\mathfrak{J}(\boldsymbol{\theta})]_{i j}=\frac{2}{\sigma^{2}} \mathfrak{R}\left(\sum_{k=0}^{K-1} \sum_{n=1}^{N} \sum_{m=1}^{M} \frac{\partial h}{\partial \theta_{i}} \frac{\partial h^{H}}{\partial \theta_{j}}\right)
$$


where the partial derivatives with respect to each parameter can be shown following straightforward derivations as

$$
\begin{aligned}
\frac{\partial h}{\partial \mathfrak{R}\left(\alpha_{p}\right)} & =e^{j\left(k \nu_{p}-(n-1) \mu_{p}^{\mathrm{r}}-(m-1) \mu_{p}^{\mathrm{t}}\right)} \\
\frac{\partial h}{\partial \mathfrak{I}\left(\alpha_{p}\right)} & =j e^{j\left(k \nu_{p}-(n-1) \mu_{p}^{\mathrm{r}}-(m-1) \mu_{p}^{\mathrm{t}}\right)} \\
\frac{\partial h}{\partial \mu_{p}^{\mathrm{r}}} & =-j(n-1) \alpha_{p} e^{j\left(k \nu_{p}-(n-1) \mu_{p}^{\mathrm{r}}-(m-1) \mu_{p}^{\mathrm{t}}\right)} \\
\frac{\partial h}{\partial \mu_{p}^{\mathrm{t}}} & =-j(m-1) \alpha_{p} e^{j\left(k \nu_{p}-(n-1) \mu_{p}^{\mathrm{r}}-(m-1) \mu_{p}^{\mathrm{t}}\right)} \\
\frac{\partial h}{\partial \nu_{p}} & =j k \alpha_{p} e^{j\left(k \nu_{p}-(n-1) \mu_{p}^{\mathrm{r}}-(m-1) \mu_{p}^{\mathrm{t}}\right)}
\end{aligned}
$$

Using (8.89) and (8.90) - (8.94), and performing some simplifications ${ }^{5}$, the FIM submatrix corresponding to the $p$ th path is obtained as

$$
\left[\mathfrak{J}\left(\boldsymbol{\theta}_{p}\right)\right]=\frac{N M K}{\sigma^{2}}\left[\begin{array}{ccccc}
2 & 0 & 0 & 0 & 0 \\
0 & 2 & 0 & 0 & 0 \\
0 & 0 & \frac{2 N^{2}}{3} & \frac{N M}{2} & -\frac{N K}{2} \\
0 & 0 & \frac{N M}{2} & \frac{2 M^{2}}{3} & -\frac{M K}{2} \\
0 & 0 & -\frac{N K}{2} & -\frac{M K}{2} & \frac{2 K^{2}}{3}
\end{array}\right]
$$

where we have assumed that $K, N$ and/or $M$ are large ${ }^{6}$. Note that under this assumption, parameter identifiability which results in rank-deficient FIM in SISO channels is very rare [189]. We also assume that the complex amplitude is Gaussian distributed $\left(\alpha_{p} \sim \mathcal{C N}(0,1)\right)$ such that $\mathbb{E}\left[\left|\alpha_{p}\right|^{2}\right]=1$ and $\mathbb{E}\left[\Re\left(\alpha_{p}\right)\right]=\mathbb{E}\left[\mathfrak{I}\left(\alpha_{p}\right)\right]=0$. Assuming that the scattering sources are uncorrelated, the FIM has a block diagonal structure

$$
\mathfrak{J}(\boldsymbol{\Theta})=\operatorname{blkdiag}\left[\mathfrak{J}\left(\boldsymbol{\theta}_{1}\right) \quad \mathfrak{J}\left(\boldsymbol{\theta}_{2}\right) \quad \cdots \quad \mathfrak{J}\left(\boldsymbol{\theta}_{P}\right)\right]
$$

\footnotetext{
${ }^{5}$ See Appendix B.6 for details of the mathematical simplifications.

${ }^{6} \mathrm{~A}$ necessary condition is that $N M Z$ is large, such that the approximation $\sum_{i=1}^{N M Z} a \approx$ $N M Z \mathbb{E}[a]$ holds.
} 
Using the structure of (8.96), the inverse can be obtained by inverting each submatrix, that is

$$
\left[\mathfrak{J}\left(\boldsymbol{\theta}_{p}\right)\right]^{-1}=\frac{\sigma^{2}}{N M K}\left[\begin{array}{ccccc}
\frac{1}{2} & 0 & 0 & 0 & 0 \\
0 & \frac{1}{2} & 0 & 0 & 0 \\
0 & 0 & \frac{21}{5 N^{2}} & -\frac{9}{5 M N} & \frac{9}{5 K N} \\
0 & 0 & -\frac{9}{5 M N} & \frac{21}{5 M^{2}} & \frac{9}{5 K M} \\
0 & 0 & \frac{9}{5 K N} & \frac{9}{5 K M} & \frac{21}{5 K^{2}}
\end{array}\right]
$$

The variance of the parameter estimates are therefore bounded by the diagonal entries of (8.96). Using the CRB for functions of parameters, the asymptotic MSEB (AMSEB) is

$$
\operatorname{AMSEB}(k)=\frac{1}{N M} \sum_{n=1}^{N} \sum_{m=1}^{M} \frac{\partial h(n, m, k)}{\partial \boldsymbol{\Theta}}[\mathfrak{J}(\boldsymbol{\Theta})]^{-1} \frac{\partial h(n, m, k)^{H}}{\partial \boldsymbol{\Theta}}
$$

Due to the diagonal structure of the FIM and independence of FIM submatrices on path parameters, the AMSEB can be written as

$$
\operatorname{AMSEB}(k)=\frac{P}{N M} \sum_{n=1}^{N} \sum_{m=1}^{M} \frac{\partial h(n, m, k)}{\partial \boldsymbol{\theta}}[\mathfrak{J}(\boldsymbol{\theta})]^{-1} \frac{\partial h(n, m, k)^{H}}{\partial \boldsymbol{\theta}}
$$

Note that for the same signal-to-noise ratio (SNR), the noise variance for a $P$ path channel is $\sigma_{P}^{2}=P \sigma^{2}$, where $\sigma^{2}$ is the noise variance for a single path channel at the same SNR. Using (8.97) and (8.99), and after some simplifications, the asymptotic MSEB is obtained as

$$
\operatorname{AMSEB}(k)=\frac{P^{2} \sigma^{2}}{5 N M K}\left(\frac{29}{2}-\frac{18 k}{K}+\frac{21 k^{2}}{K^{2}}\right)
$$

for $k=0,1,2, \cdots$, where $k \in(0, K-1)$ corresponds to the estimation/ measurement segment and prediction starts at $k=K$. In this form, (8.100) provide useful insights into the effects of the number of antennas, the number of paths, SNR and the number of samples on the MSEB. The first constant term is the contribution from time independent parameters (i.e., amplitude, $\mathrm{AOA}$ and $\mathrm{AOD}$ ) and their cross terms, the second term results from the cross 
terms involving the Doppler frequency and the third, quadratic term is the contribution from the Doppler frequency estimation. This quadratic growth of this term emphasizes the need for accurate estimation of the Doppler frequencies.

\subsubsection{Wideband Channels}

We now present a derivation of a closed-form asymptotic error bound for pilot based wideband MIMO systems. Consider the doubly-selective channel in (8.28), the entries of which can be represented as

$$
h(n, m, k, q)=\sum_{p=1}^{P} \alpha_{p} e^{j\left(k \nu_{p}-(n-1) \mu_{p}^{\mathrm{r}}-(m-1) \mu_{p}^{\mathrm{t}}-q \eta_{p}\right)}
$$

for all $n=1, \cdots, N, m=1, \cdots, M, k=0, \cdots, P-1$ and $q=0,1, \cdots, Q-1$. We will henceforth remove the indices in parenthesis and denote $h(n, m, p, q)$ as $h$. Let the channel parameter vector be denoted by ${ }^{7}$

$$
\Theta=\left[\boldsymbol{\theta}_{1}, \boldsymbol{\theta}_{2}, \cdots, \boldsymbol{\theta}_{P}\right]
$$

where

$$
\boldsymbol{\theta}_{p}=\left[\begin{array}{llllll}
\Re\left(\alpha_{p}\right) & \mathfrak{I}\left(\alpha_{p}\right) & \mu_{p}^{\mathrm{r}} & \mu_{p}^{\mathrm{t}} & \nu_{p} & \eta_{p}
\end{array}\right]
$$

Using (8.38), the Mean squared error bound (MSEB) can be expressed as

$$
\operatorname{MSEB}(k, q)=\sum_{n=1}^{N} \sum_{m=1}^{M} \frac{\partial h}{\partial \boldsymbol{\Theta}}[\mathfrak{J}(\boldsymbol{\Theta})]^{-1} \frac{\partial h^{H}}{\partial \boldsymbol{\Theta}}
$$

where $\operatorname{MSEB}(k, q)=\mathbb{E}\left[(\hat{\mathbf{h}}(k, q)-\mathbf{h}(k, q))^{H}(\hat{\mathbf{h}}(k, q)-\mathbf{h}(k, q))\right], \mathfrak{J}^{-1}(\boldsymbol{\Theta})$ is the CRLB on the variance of the channel parameter estimates. The Jacobian in (8.104) is given by

$$
\frac{\partial h}{\partial \boldsymbol{\Theta}}=\left[\begin{array}{llll}
\frac{\partial h}{\partial \boldsymbol{\theta}_{1}} & \frac{\partial h}{\partial \boldsymbol{\theta}_{2}} & \cdots & \frac{\partial h}{\partial \boldsymbol{\theta}_{P}}
\end{array}\right]
$$

\footnotetext{
${ }^{7}$ Note that although the noise variance $\sigma^{2}$ can also be included as an element of $\boldsymbol{\Theta}$, it is omitted here since this does not affect the expression for the prediction error bound. A proof of the equivalence of the prediction error bound with and without noise variance in the parameter set can be found in Appendix B.5.
} 
Using (8.13), the FIM can be defined element-wise as

$$
[\mathfrak{J}(\boldsymbol{\Theta})]_{i j}=\frac{2}{\sigma^{2}} \mathfrak{R}\left(\sum_{q=0}^{Q-1} \sum_{k=0}^{K-1} \sum_{n=1}^{N} \sum_{m=1}^{M} \frac{\partial h}{\partial \boldsymbol{\Theta}_{i}} \frac{\partial h^{H}}{\partial \boldsymbol{\Theta}_{j}}\right)
$$

Following straightforward derivation, the partial derivatives with respect to each of the parameters can be shown to be

$$
\begin{aligned}
\frac{\partial h}{\partial \mathfrak{R}\left(\alpha_{p}\right)} & =e^{j\left(k \nu_{p}-(n-1) \mu_{p}^{\mathrm{r}}-(m-1) \mu_{p}^{\mathrm{t}}-q \eta_{p}\right)} \\
\frac{\partial h}{\partial \mathfrak{I}\left(\alpha_{p}\right)} & =j e^{j\left(k \nu_{p}-(n-1) \mu_{p}^{\mathrm{r}}-(m-1) \mu_{p}^{\mathrm{t}}-q \eta_{p}\right)} \\
\frac{\partial h}{\partial \mu_{p}^{\mathrm{r}}} & =-j(n-1) \alpha_{k} e^{j\left(k \nu_{p}-(n-1) \mu_{p}^{\mathrm{r}}-(m-1) \mu_{p}^{\mathrm{t}}-q \eta_{p}\right)} \\
\frac{\partial h}{\partial \mu_{p}^{\mathrm{t}}} & =-j(m-1) \alpha_{z} e^{j\left(k \nu_{p}-(n-1) \mu_{p}^{\mathrm{r}}-(m-1) \mu_{p}^{\mathrm{t}}-q \eta_{p}\right)} \\
\frac{\partial h}{\partial \nu_{p}} & =j k U_{t} \alpha_{p} e^{j\left(k \nu_{p}-(n-1) \mu_{p}^{\mathrm{r}}-(m-1) \mu_{p}^{\mathrm{t}}-q \eta_{p}\right)} \\
\frac{\partial h}{\partial \eta_{p}} & =-j q U_{f} \alpha_{p} e^{j\left(k \nu_{p}-(n-1) \mu_{p}^{\mathrm{r}}-(m-1) \mu_{p}^{\mathrm{t}}-q \eta_{p}\right)}
\end{aligned}
$$

Using (8.106)-(8.112) and performing some simplifications, the FIM submatrix corresponding to the $p$ th path is obtained as

$$
\mathfrak{J}\left(\boldsymbol{\theta}_{p}\right)=\frac{N M K Q}{\sigma^{2}} \mathfrak{K}
$$

with

$$
\mathfrak{K}=\left[\begin{array}{cccccc}
2 & 0 & 0 & 0 & 0 & 0 \\
0 & 2 & 0 & 0 & 0 & 0 \\
0 & 0 & \frac{2 N^{2}}{3} & \frac{N M}{2} & -\frac{N K U_{t}}{2} & \frac{N Q U_{f}}{2} \\
0 & 0 & \frac{N M}{2} & \frac{2 M^{2}}{3} & -\frac{M K U_{t}}{2} & \frac{M Q U_{f}}{2} \\
0 & 0 & -\frac{N K U_{t}}{2} & -\frac{M K U_{t}}{2} & \frac{2 K^{2} U_{t}^{2}}{3} & -\frac{Q K U_{t} U_{f}}{2} \\
0 & 0 & \frac{N Q U_{f}}{2} & \frac{M Q U_{f}}{2} & -\frac{Q K U_{t} U_{f}}{2} & \frac{2 Q^{2} U_{f}^{2}}{3}
\end{array}\right]
$$

where we have assumed that $K, Q, N$ and/or $M$ are large ${ }^{8}$ and that the complex amplitude is $\alpha_{z} \sim \mathcal{C N}(0,1)$, such that $\mathbb{E}\left[\left|\alpha_{z}\right|^{2}\right]=1$ and $\mathbb{E}\left[\Re\left(\alpha_{z}\right)\right]=$

\footnotetext{
${ }^{8}$ It should be noted that $K, Q, N$ and $M$ do not all have to be large. We only require $N M K Q$ to be fairly large so that the approximation $N M K Q \mathbb{E}[g] \approx \sum_{i=1}^{N M K Q} g$ holds.
} 
$\mathbb{E}\left[\Im\left(\alpha_{z}\right)\right]=0$. Using the structure of (8.113), the inverse of the FIM submatrix is given by

$$
\left[\mathfrak{J}\left(\boldsymbol{\theta}_{p}\right)\right]^{-1}=\frac{\sigma^{2}}{N M K Q} \mathfrak{K}^{-1}
$$

where $\mathfrak{K}^{-1}$ is the inverse of $\mathfrak{K}$ given by

$$
\mathfrak{K}^{-1}=\left[\begin{array}{cccccc}
\frac{1}{2} & 0 & 0 & 0 & 0 & 0 \\
0 & \frac{1}{2} & 0 & 0 & 0 & 0 \\
0 & 0 & \frac{60}{13 N^{2}} & \frac{-18}{13 M N} & \frac{18}{13 N K U_{t}} & \frac{-18}{13 N Q U_{f}} \\
0 & 0 & \frac{-18}{13 M N} & \frac{60}{13 M^{2}} & \frac{18}{13 M K U_{t}} & \frac{-18}{13 M Q U_{f}} \\
0 & 0 & \frac{18}{13 N K U_{t}} & \frac{18}{13 M K U_{t}} & \frac{60}{13 K^{2} U_{t}^{2}} & \frac{18}{13 P Q U_{t} U_{f}} \\
0 & 0 & \frac{-18}{13 N Q U_{f}} & \frac{-18}{13 M Q U_{f}} & \frac{18}{13 K Q U_{t} U_{f}} & \frac{60}{13 Q^{2} U_{f}^{2}}
\end{array}\right]
$$

The asymptotic mean square error bound (AMSEB) can now be written as

$$
\operatorname{AMSEB}(k, q)=\sum_{n=1}^{N} \sum_{m=1}^{M} \frac{\partial h}{\partial \boldsymbol{\Theta}}[\mathbf{J}(\boldsymbol{\Theta})]^{-1}{\frac{\partial h^{H}}{\partial \boldsymbol{\Theta}}}^{H}
$$

Again, we define the signal-to-noise ratio $(\mathrm{SNR})$ as ${ }^{9} \mathrm{SNR}=P / \sigma_{P}^{2}$. Thus, at the same SNR, the noise variance for a $P$-path channel is $\sigma_{P}^{2}=P \sigma^{2}$, where $\sigma^{2}$ is the noise variance for a single path channel. Substituting (8.115) into (8.117) and performing some simplifications, we obtain

$$
\begin{gathered}
\operatorname{AMSEB}(k, q)=\frac{P^{2} \sigma^{2}}{13 K Q}\left[44-\frac{36 k}{K U_{t}}+\frac{60 k^{2}}{K^{2} U_{t}^{2}}-\frac{36 q}{Q U_{f}}\right. \\
\left.+\frac{60 q^{2}}{Q^{2} U_{f}^{2}}-\frac{36 q k}{K^{2} U_{t}^{2} Q^{2} U_{f}^{2}}\right]
\end{gathered}
$$

Based on the assumption of normally distributed complex amplitudes, it can be shown that for a $P$-path channel $\mathbb{E}\left[\|\mathbf{H}\|_{F}^{2}\right]=N M K$ and the asymptotic

\footnotetext{
${ }^{9}$ This definition is necessary in order to allow fair comparison of the bound across channels with different number of paths
} 
normalized mean square error bound (ANMSEB) is obtained from (8.118) as

$$
\begin{array}{r}
\operatorname{ANMSEB}(k, q)=\frac{P \sigma^{2}}{13 N M K Q}\left[44-\frac{36 k}{K U_{t}}+\frac{60 k^{2}}{K^{2} U_{t}^{2}}\right. \\
\left.-\frac{36 q}{Q U_{f}}+\frac{60 q^{2}}{Q^{2} U_{f}^{2}}-\frac{36 k q}{Q U_{f} K U_{t}}\right]
\end{array}
$$

In this form, the ANMSEB provides useful insights on the effects of the number of antennas, number of frequency and time domain pilots, pilot spacing and SNR on the estimation, interpolation and prediction performance. The following observations can be made from (8.119):

- Since the ANMSEB is quadratic in the frequency index and has a minimum at the center of the band (see the frequency axis of Fig. 8.5), the subcarriers near the edge of the frequency band are less predictable than those near the center.

- The NMSE grows linearly with an increasing noise variance $\sigma^{2}$ and number of propagation paths $P$. This is intuitive and agrees with previous results that prediction becomes more difficult with increasing number of paths $[189,190]$.

- The NMSE decreases with increasing number of antennas at either or both ends of the link. This is also intuitive since more structure of the channel is revealed by having more antennas.

- The contribution to the NMSE from the Doppler frequency (see (8.111), (8.119)) and delay estimation (see (8.112), (8.119))) lead to the $p^{2}$ and $q^{2}$ terms, respectively, demonstrating a quadratic increase with prediction horizon and with frequency. This shows the need to accurately estimate the Doppler frequency and path delays for spatial/temporal prediction and frequency domain interpolation, respectively.

- The contributions from the cross correlation of error terms involving the Doppler frequency lead to the negative linear term in $k$ in (8.118), 
thus reducing the ANMSEB. An explanation (see the illustration in Section 4.4) for this is that improved Doppler frequency estimates can be obtained from joint parameter estimation. A similar term is obtained from cross terms involving the delays.

\subsubsection{D Asymptotic Prediction Error Bound}

In this section, we derive a close-form expression for the prediction error bound in 3D channels with a UPA at the BS and a ULA at the MS. Consider the 3D channel model in (4.114), the entries of which can be expressed as

$$
h\left(n_{x}, n_{y}, m, k\right)=\sum_{p=1}^{P} \alpha_{p} e^{j\left\{\left(n_{x}-1\right) \mu_{p}^{\mathrm{tx}}+\left(n_{y}-1\right) \mu_{p}^{\mathrm{ty}}+(m-1) \mu_{p}^{\mathrm{r}}+k \nu_{p}\right\}}
$$

Using (8.12), the 3D FIM can be expressed as

$$
\mathfrak{J}=\frac{2}{\sigma^{2}} \mathfrak{R}\left[\sum_{k=0}^{K-1} \sum_{n_{x}=1}^{N_{x}} \sum_{n_{y}=1}^{N_{y}} \sum_{m=1}^{M} \frac{\partial h^{H}\left(n_{x}, n_{y}, m\right)}{\partial \boldsymbol{\Theta}} \frac{\partial h\left(n_{x}, n_{y}, m\right)}{\partial \boldsymbol{\Theta}}\right]
$$

As in the 2D case, we let the parametrization of the channel be

$$
\Theta=\left[\begin{array}{llll}
\boldsymbol{\theta}_{1} & \boldsymbol{\theta}_{2} & \cdots & \boldsymbol{\theta}_{P}
\end{array}\right]
$$

where $\boldsymbol{\theta}_{p}=\left[\mathfrak{R}\left(\alpha_{p}\right), \mathfrak{I}\left(\alpha_{p}\right), \mu_{p}^{\mathrm{r}}, \mu_{p}^{\mathrm{tx}}, \mu_{p}^{\mathrm{ty}}, \nu_{p}\right]$. Using (8.120), the derivatives of the $3 \mathrm{D}$ channel with respect to the parameters of the $p$ th path can be shown 
to be

$$
\begin{aligned}
\frac{\partial h}{\partial \mathfrak{R}\left(\alpha_{p}\right)} & =e^{j\left\{\left(n_{x}-1\right) \mu_{p}^{\mathrm{tx}}+\left(n_{y}-1\right) \mu_{p}^{\mathrm{ty}}+(m-1) \mu_{p}^{\mathrm{r}}+k \nu_{p}\right\}} \\
\frac{\partial h}{\partial \mathfrak{I}\left(\alpha_{p}\right)} & =j e^{j\left\{\left(n_{x}-1\right) \mu_{p}^{\mathrm{tx}}+\left(n_{y}-1\right) \mu_{p}^{\mathrm{ty}}+(m-1) \mu_{p}^{\mathrm{r}}+k \nu_{p}\right\}} \\
\frac{\partial h}{\partial \mu_{p}^{\mathrm{r}}} & =j(m-1) \alpha_{p} e^{j\left\{\left(n_{x}-1\right) \mu_{p}^{\mathrm{tx}}+\left(n_{y}-1\right) \mu_{p}^{\mathrm{ty}}+(m-1) \mu_{p}^{\mathrm{r}}+k \nu_{p}\right\}} \\
\frac{\partial h}{\partial \mu_{p}^{\mathrm{tx}}} & =j\left(n_{x}-1\right) \alpha_{p} e^{j\left\{\left(n_{x}-1\right) \mu_{p}^{\mathrm{tx}}+\left(n_{y}-1\right) \mu_{p}^{\mathrm{ty}}+(m-1) \mu_{p}^{\mathrm{r}}+k \nu_{p}\right\}} \\
\frac{\partial h}{\partial \mu_{p}^{\mathrm{ty}}} & =j\left(n_{y}-1\right) \alpha_{p} e^{j\left\{\left(n_{x}-1\right) \mu_{p}^{\mathrm{ty}}+\left(n_{y}-1\right) \mu_{p}^{\mathrm{ty}}+(m-1) \mu_{p}^{\mathrm{r}}+k \nu_{p}\right\}} \\
\frac{\partial h}{\partial \nu_{p}} & =j k \alpha_{p} e^{j\left\{\left(n_{x}-1\right) \mu_{p}^{\mathrm{ty}}+\left(n_{y}-1\right) \mu_{p}^{\mathrm{ty}}+(m-1) \mu_{p}^{\mathrm{r}}+k \nu_{p}\right\}}
\end{aligned}
$$

In the asymptotic limit of large $N_{x}, N_{y}, M$ and/or $K$, the FIM for the $p$ th path is obtained using (8.121) and (8.123) as

$$
\mathfrak{J}_{p}=\frac{N_{x} N_{y} M K}{\sigma^{2}}\left[\begin{array}{cccccc}
2 & 0 & 0 & 0 & 0 & 0 \\
0 & 2 & 0 & 0 & 0 & 0 \\
0 & 0 & \frac{2 M^{2}}{3} & \frac{M N_{x}}{2} & \frac{M N_{y}}{2} & \frac{M K}{2} \\
0 & 0 & \frac{M N_{x}}{2} & \frac{2 N_{x}^{2}}{3} & \frac{N_{x} N_{y}}{2} & \frac{N_{x} K}{2} \\
0 & 0 & \frac{M N_{y}}{2} & \frac{N_{x} N_{y}}{2} & \frac{2 N_{y}^{2}}{3} & \frac{N_{y} K}{2} \\
0 & 0 & \frac{M K}{2} & \frac{N_{x} K}{2} & \frac{N_{y} K}{2} & \frac{2 K^{2}}{3}
\end{array}\right]
$$

Here, we assumed that the complex amplitudes are normally distributed: $\alpha_{p} \sim \mathcal{C N}(0,1)$. On inverting (8.129), we obtain the asymptotic bound on the parameter estimates covariance as

$$
\mathfrak{J}_{p}^{-1}=\frac{\sigma^{2}}{N_{x} N_{y} M K}\left[\begin{array}{cccccc}
\frac{1}{2} & 0 & 0 & 0 & 0 & 0 \\
0 & \frac{1}{2} & 0 & 0 & 0 & 0 \\
0 & 0 & \frac{60}{13 M^{2}} & \frac{-18}{13 M N_{x}} & \frac{-18}{13 M N_{y}} & \frac{-18}{13 M K} \\
0 & 0 & \frac{-18}{13 M N_{x}} & \frac{60}{13 N_{x}^{2}} & \frac{-18}{13 N_{x} N_{y}} & \frac{-18}{13 K N_{x}} \\
0 & 0 & \frac{-18}{13 M N_{y}} & \frac{-18}{13 N_{x} N_{y}} & \frac{60}{13 N_{y}^{2}} & \frac{-18}{13 K N_{y}} \\
0 & 0 & \frac{-18}{13 M K} & \frac{-18}{13 K N_{x}} & \frac{-18}{13 K N_{y}} & \frac{60}{13 K^{2}}
\end{array}\right]
$$


After some simplifications, the asymptotic 3D error bound is obtained as

$$
\operatorname{AMSEB}(\ell)=\frac{P \sigma^{2}}{13 K}\left[46-\frac{54 k}{K}+\frac{60 k^{2}}{K^{2}}\right]
$$

\subsection{Numerical Simulations}

In this section, we perform experiments to study the effects of system parameters on the error bounds. The asymptotic bounds are also compared with the bound. For clarity, results for narrowband and wideband channels are presented separately. For our simulations, we utilized the normalized MSEB (NMSEB) defined as NMSEB $=\mathrm{MSEB} / P$. The NMSE bound is averaged over 500 independent realizations of the channel. The channel parameters are generated for each realization as follows. The complex amplitudes are randomly drawn from a complex Gaussian distribution as $\alpha_{p} \sim \mathcal{C N}(0,1)$. The angles of arrival and departure are both selected from a uniform distribution as $\theta_{p}^{\mathrm{r}}, \theta_{p}^{\mathrm{t}} \sim \mathcal{U}[-\pi, \pi)$. The Doppler frequencies are generated from a spatial rather than temporal point of view as $\nu_{p}=2 \pi \Delta x \sin \theta_{p}^{\mathrm{v}}$, where $\Delta x$ is the spatial sampling interval in wavelengths and $\theta_{p}^{\mathrm{v}}$ is the angle between the direction of travel of the mobile station and the receive antenna array. We also select $\theta_{p}^{\mathrm{v}}$ from a uniform distribution as $\theta_{p}^{\mathrm{v}} \sim \mathcal{U}[-\pi, \pi)$. The path delays are selected from the delays for the Urban macro (UMA) scenario in the WINNER II/3GPP channel [3]. Except where otherwise stated, we consider a sampling rate of 10 samples per wavelength.

\subsubsection{Narrowband MIMO Channel}

Fig. 8.1 presents the NMSE bounds for a two path channel with $K=50$, $N=2$ and $M=2$ versus estimated and predicted sample index and compares these with the performance of the DOD/DOA-MEMCHAP in Section 4.4.4. With the sampling interval used for the NMSE bound computation and MEMCHAP prediction, this corresponds to a measurement length of $5 \lambda$ and 
prediction segment of $3 \lambda$. As seen from the figure, the NMSE bounds increase quadratically with increasing prediction horizon and the asymptotic bound agrees closely with the bound. In Fig. 8.2, we plot the NMSE bounds versus the number of paths for a prediction horizon of 10 samples $(1 \lambda)$. We observe that the NMSE bounds increase with increasing number of paths. This agrees with previous observations that propagation channels having dense multipath are more difficult to predict $[189,190]$. Fig. 8.3 shows the effect of the number of antenna at the transmit and receive ends of the MIMO link on the prediction NMSE bound using the asymptotic error bound. As can be seen, the NMSE decreases with increasing number of antennas at either or both ends. We plot the NMSE bound versus the number of samples in the observation segment for a $2 \times 2$ channel with $P=2$ in Fig. 8.4. We observe that the NMSE decreases with increasing number of samples. This is intuitively predictable since an increased number of samples leads to improved parameter estimation and hence, better prediction. Using a criterion similar to that in [189], a predicted sample is useful if NMSE $\leq 0.05(\approx-13 \mathrm{~dB})$. Similar results are obtained for the 3D channels.

\subsubsection{Wideband MIMO Channels}

We study the effects of system parameters on the wideband MIMO prediction error bounds and compare the asymptotic bound in (8.27) with the results in $[109,110]$. In order to be consistent with $[109,110]$, we consider the root normalized mean square error (RNMSE) defined as RNMSE $=\sqrt{\mathrm{NMSE}}$. We consider a MIMO-OFDM system with bandwidth $B=20 \mathrm{MHz}$, number of subcarriers $N_{\mathrm{sc}}=2048$ and with 64 equally spaced pilot subcarriers. We assume that the channel is sampled at every symbol duration $\left(U_{\mathrm{t}}=1\right)$.

Fig. 8.5 presents a plot of the asymptotic bound and the bounds in [109, 110] for a two path channel with $K=100, Q=64, N=2, M=2$ and $\mathrm{SNR}=$ $15 \mathrm{~dB}$ as a function of frequency and horizon (in wavelengths). The blue surface is the bound and the red surface is the asymptotic approximation. 
As seen from the figure, the NMSE bounds increase quadratically with both frequency and temporal horizon and the asymptotic bound approximates the bound very closely.

In Fig. 8.6, we plot the RNMSE bounds averaged over frequency versus prediction horizon for $\mathrm{SNR}$ of $0 \mathrm{~dB}$ and $5 \mathrm{~dB}$. We observe that over the range considered, the maximum difference between the bounds in $[109,110]$ and our approximation is only about $0.3 \mathrm{~dB}$. As expected the bounds increase with horizon but decreases with increasing SNR. The difference between the asymptotic approximation and the bound is dependent on the value of the product $N M K Q$ as illustrated in Figs. 8.7-8.10, where we present similar results for different values of $N, M, K$ and $Q$. We observed that as the product increases, the asymptotic approximation more closely approximates to the bound.

We plot the RNMSE bound versus the number of samples in the observation segment in Fig. 8.11 for different numbers of antenna elements at both ends of the link. We observe that the RNMSE decreases with increasing number of samples. This is because an increased number of samples leads to improved parameter estimation and hence to better prediction. It also shows that an increase in the number of transmit and/or receive antenna decreases the RNMSE. Since the asymptotic bound becomes looser as the product $N M K Q$ decreases, it is advisable to use the error bound expression for values of $N \leq 2, M \leq 2, K \leq 5$, and $Q \leq 4$.

Finally, we show the effects of the number of paths on RNMSE in Fig. 8.12. We observe the the RNMSE bounds increases with increasing numbers of paths. This agrees with previous observations that propagation channels with dense multipath are more difficult to predict $[189,190]$.

\subsection{Summary}

In this chapter, we derived expressions for the lower bound on prediction error in narrowband, wideband, polarized and 3D mobile MIMO channels. 
We considered pilot based systems with UPA and ULA at the BS for the $2 \mathrm{D}$ and $3 \mathrm{D}$ scenarios, respectively. A ULA at the MS is considered in both cases.

We also derived simple, readily interpretable and insightful closed-form expressions for the lower bounds on the performance of channel estimation, interpolation and prediction. The bound is obtained using the vector formulation of the Cramer Rao bound for functions of parameters in the asymptotic limits of large frequency and/or time-domain training samples and number of antennas. The expressions provide useful insights into the effects of system design parameters such as the number of antennas, number of training pilots, noise level, number of paths and pilot spacing on the error performance and are independent of the actual channel parameters. Simulation results show that the asymptotic error bounds provide good approximations to the bound while eliminating the need for repeated computation. 


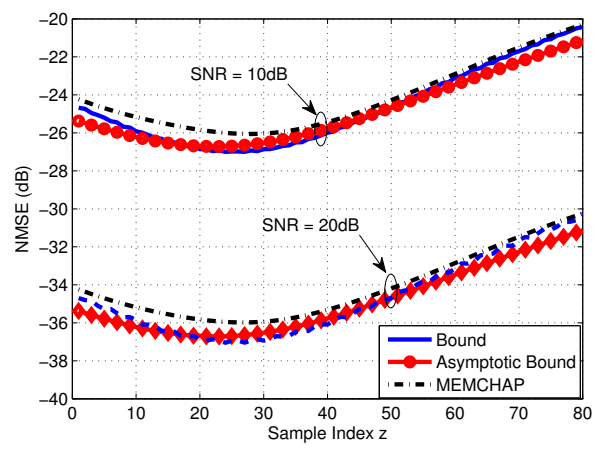

Figure 8.1: NMSEB versus sample index with $Z=50, N=2$ and $M=2$.

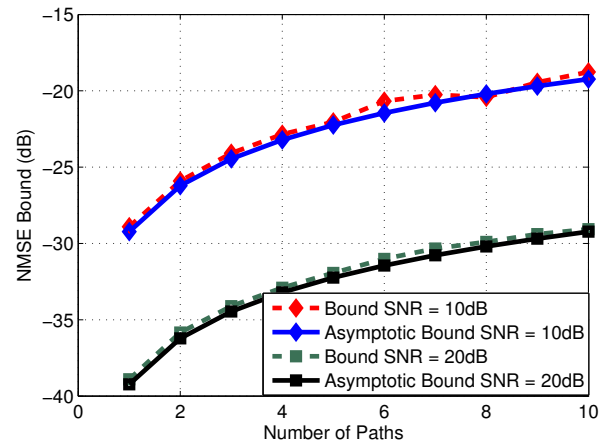

Figure 8.2: NMSEB versus $P$ for a horizon of 10 sampling intervals.

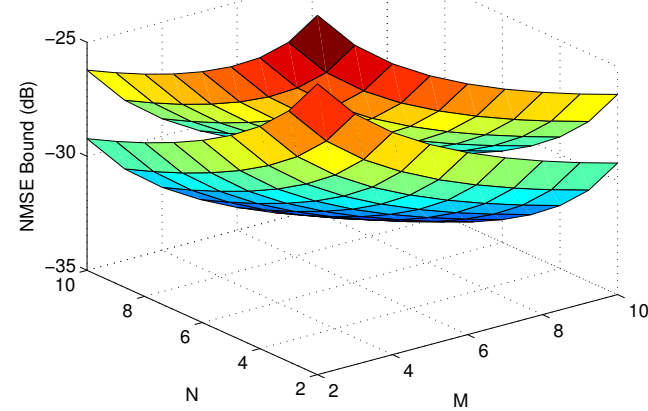

Figure 8.3: NMSEB versus number of antennas for with $k=10, Z=50$ and $P=5$.

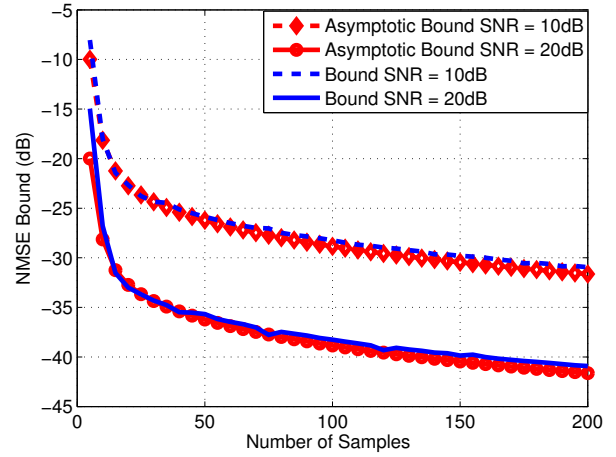

Figure 8.4: NMSEB versus number of samples.

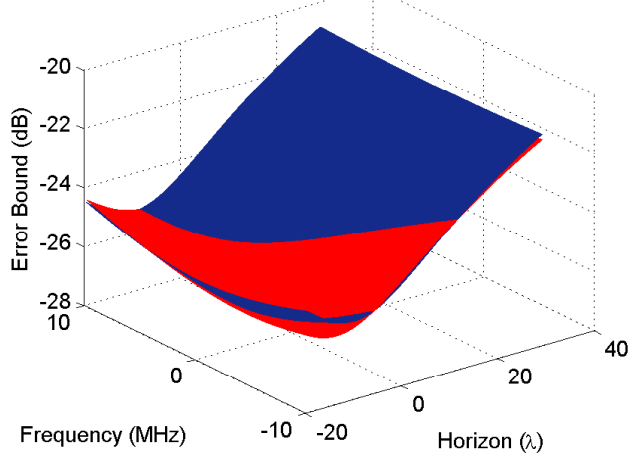

Figure 8.5: RNMSE versus frequency and horizon $(\lambda)$.

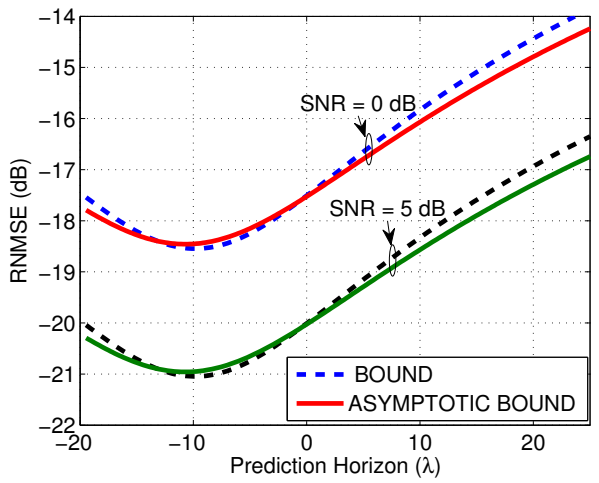


Figure 8.6: Averaged RNMSE versus Figure 8.9: Averaged RNMSE with horizon $(\lambda)$.
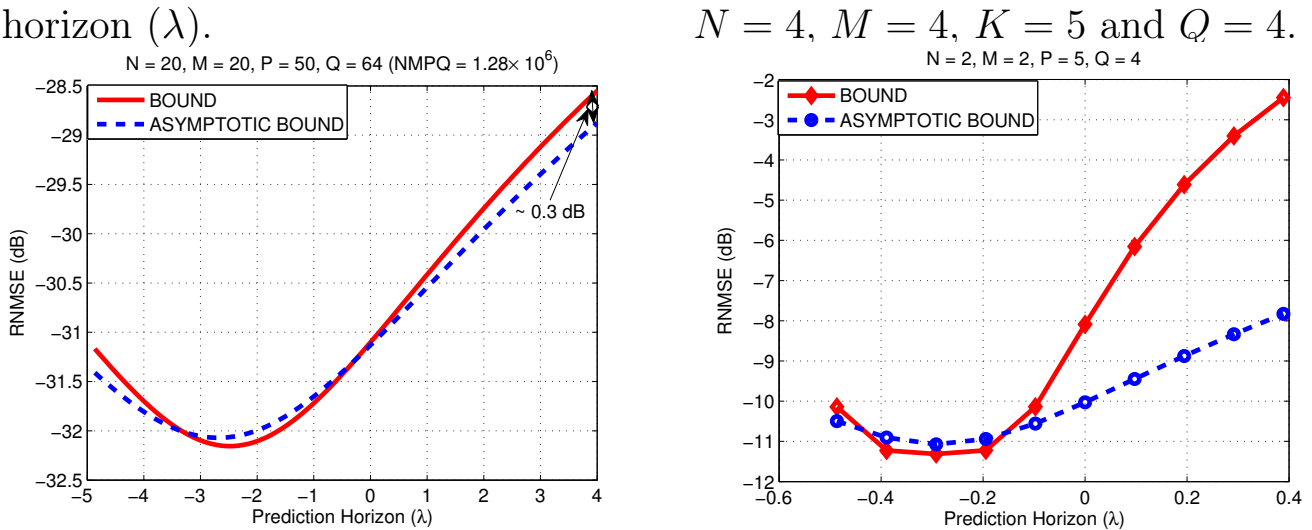

Figure 8.7: Averaged RNMSE with $N=20, M=20, K=50$ and

Figure 8.10: Averaged RNMSE with $Q=64$.

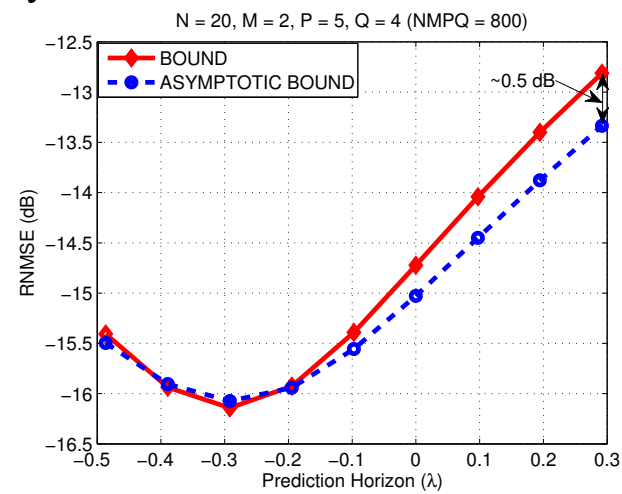

$N=2, M=2, K=5$ and $Q=4$.

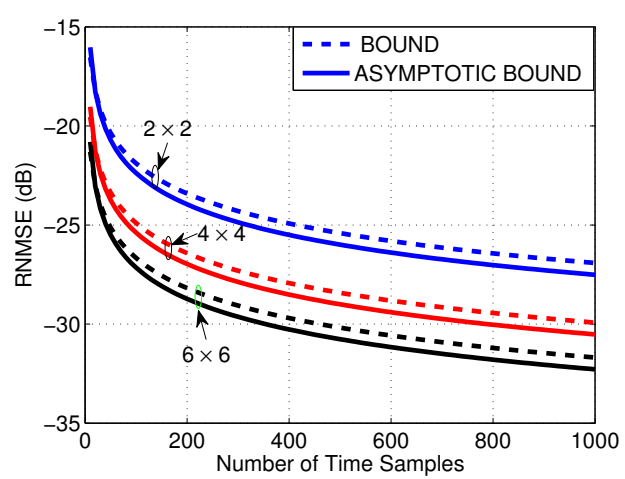

Figure 8.11: Averaged RNMSE ver-

Figure 8.8: Averaged RNMSE with $N=20, M=2, K=5$ and $Q=4$.

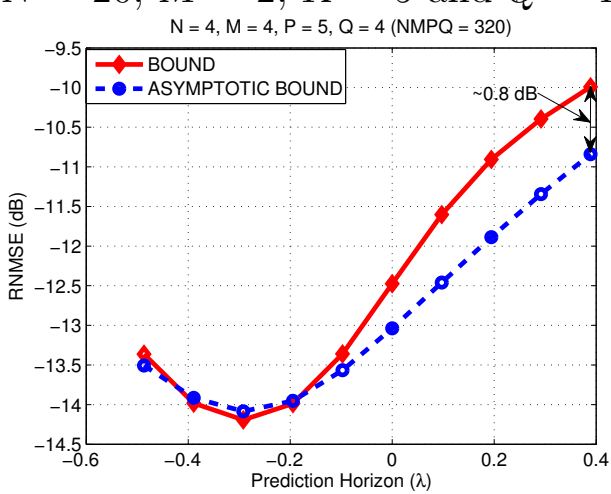

sus number of training samples.

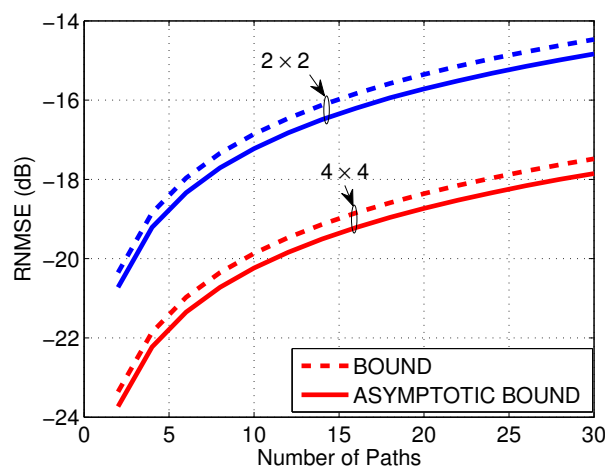

Figure 8.12: Averaged RNMSE ver200 sus number of paths. 


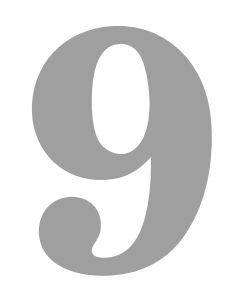

\title{
Dynamic Model Based Prediction and Parameter Tracking
}

\begin{abstract}
$\mathrm{T}^{\mathrm{N}}$ the previous chapters, we developed several schemes for the prediction 1 of narrowband and wideband MIMO channels based on parametric radio channel (PRC) modeling. The proposed methods assume that the underlying propagation parameters are stationary over the region considered. However, these parameters may exhibit some variations in practice. There is therefore a need to track the spatial/temporal evolution of the parameters. Moreover, the computational complexity of the prediction schemes can be significantly reduced by eliminating the need for repeated eigenvalue decomposition and matrix inversions in the proposed method. A potential approach is to utilize low complexity subspace tracking schemes such as PAST [211,212] and Bi-iteration SVD $[155,185,186]$. Other methods utilize Bayesian schemes for recursive estimation such as the Kalman Filter (KF) [94], Extended Kalman Filter (EKF) [25] and Particle Filter (PF) [28]. While there has been extensive literature on the prediction and tracking of MIMO channels using
\end{abstract}


Bayesian approaches, particularly the KF in conjunction with Autoregressive Model (AR) models (see. e.g $[67,102,116,209]$ ), there exist few results on the application of these methods to the joint tracking of MIMO multipath parameters.

The focus of this chapter is to investigate methods for jointly tracking the parameters of MIMO channels and channel extrapolation taking account for the temporal/spatial dynamics of the underlying propagation environment.

\subsection{Dynamic Prediction Model}

In this section we introduce narrowband and wideband MIMO channels with time-varying parameters and present an overview of selected methods for modeling the dynamics of the parameters.

\subsubsection{Narrowband MIMO Channel}

Without the parameter stationarity assumption, the MIMO model in (4.2) becomes

$$
\mathbf{H}(t)=\sum_{p=1}^{P(t)} \alpha_{p}(t) \mathbf{a}_{\mathrm{r}}\left(\theta_{p}(t)\right) \mathbf{a}_{\mathbf{t}}^{T}\left(\phi_{p}(t)\right) e^{j \omega_{p}(t) t}
$$

The sampled version of (9.1) at time/spatial instant $k$ can be written as

$$
\mathbf{H}(k)=\sum_{p=1}^{P(k)} \alpha_{p}(k) \mathbf{a}_{\mathrm{r}}\left(\theta_{p}(k)\right) \mathbf{a}_{\mathrm{t}}^{T}\left(\phi_{p}(k)\right) e^{j k \nu_{p}(k)}
$$

Unlike the static channel model in (4.2), the parameters $\left\{\alpha_{p}(k) \theta_{p}(k) \phi_{p}(k) \nu_{p}\right\}_{p=1}^{P(k)}$ are all time-varying. We will however assume that the number of paths remains constant ${ }^{1}$ in our simulations. The development of scheme for tracking the complex amplitudes will be addressed in our future works.

\footnotetext{
${ }^{1}$ This assumption is made for simplicity reasons. The number of paths can be treated as time varying and methods for modeling and tracking the birth and death of scatterers can be developed.
} 


\subsubsection{Wideband MIMO Channel}

Similar to the dynamic narrowband channel in (9.2), the sampled wideband channel for $l$ th subcarrier at time instant $k$ can be expressed as

$$
\mathbf{H}(k, \ell)=\sum_{p=1}^{P(k)} \alpha_{p} \mathbf{a}_{\mathrm{r}}\left(\theta_{p}(k)\right) \mathbf{a}_{\mathrm{t}}^{T}\left(\phi_{p}(k)\right) e^{j\left[k \nu_{p}(k)-j \ell \eta_{p}(k)\right]}
$$

\subsubsection{Modeling Temporal Variation of Channel Param- eters}

Now we will describe methods for modeling the dynamics of the parameters in (9.2) and (9.3). As described in [3,79], the appearance of new scattering sources can be modeled as a homogeneous Poisson process with transition rate $\lambda_{\text {birth }}$ and the lifetime of the scatterers can be described by an exponential random variable with mean $1 / \lambda_{\text {life }}$. The number of active scatterers $P(t)$ at a given time instant is therefore a Poisson distributed random variable with mean $\left.\mathbb{E}[P(t)]=\lambda_{\text {birth }} / \lambda_{\text {life }}\right]$. An illustration of the evolution of number of scatterers is shown in Fig. 9.1, where we have used $\lambda_{\text {birth }}=10 \lambda$ and $\lambda_{\text {life }}=2 \lambda$. The average number of paths is therefore, $\mathbb{E}[P(t)]=5$.

The dynamics of the structural parameters resulting from the movement of the mobile station and/or scatterers can be described using different models depending on the environment and the rate of motion. In our analysis, we consider two common models that are often used in time series analysis. The models are described as follows.

- First-order Autoregressive Model: The dynamics of a parameter $\theta$ can be defined using the AR(1) model:

$$
\theta(k)=\beta_{\theta} \theta(k-1)+v(k)
$$

where $\beta_{\theta}$ controls the spatial/temporal variation of $\theta$ from a time instant to another and $v(k)$ is a Gaussian random variable with zero 


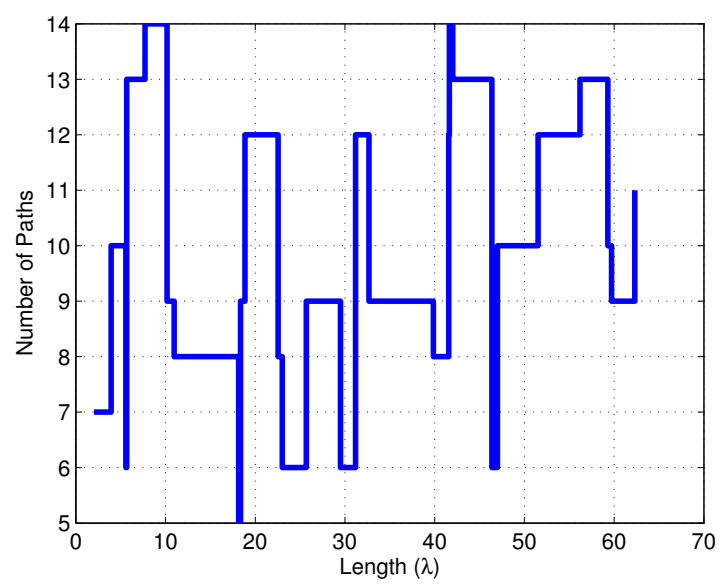

Figure 9.1: Evolution of the number of active scatterers.

mean and variance $\sigma_{\mathrm{v}}^{2}$. The $\mathrm{AR}(1)$ model was used in [86] to model time variation of path delays.

- Linear Advancement Model: An alternative approach to modeling the time variation for the parameters to follow a straight line advancement thus

$$
\theta_{p}(k)=\theta_{p}(0)+(k-1) T_{\mathrm{samp}} \beta_{\theta}
$$

Here, $\beta_{\theta}$ defines the slope of the straight line and is typically determined by the rate of movements in the scattering medium. This approach was used in [90] for modeling delay variation in SISO channels where $\beta_{\theta}$ was defined in terms of the Doppler frequency as

$$
\beta_{\theta}=\frac{\omega_{p}}{\omega_{c}}
$$

\subsection{Adaptive MIMO Prediction}

In this section, we present a framework for prediction of narrowband MIMO channels with slowly varying channel parameters. Unlike the methods presented in previous chapters, we relax the parameter stationarity assumptions 
in the adaptive prediction methods. We begin this section by illustrating the effects of varying parameters on the performance of the DOD/DOAMEMCHAP in Chapter 4. Based on the PAST subspace tracker in 3.3.1, we derive a scheme for jointly tracking the structural parameters (i.e AOD, AOA and Doppler frequency) of the channel and predicting the CSI based on the evolution of the parameters. While it may be necessary to also allow the complex amplitudes of the propagation paths to be time-varying, we retain the assumption of constant amplitudes over a specified region.

\subsubsection{Effects of Parameter variation on CSI Prediction}

The effects of slow parameter variation on the performance of the DOD/DOAMEMCHAP is illustrated in Fig. 9.2 where we plot the NMSE versus SNR at a prediction horizon of $1 \lambda$. As shown in the figure, variation of the parameters degrades the the prediction performance. This is expected, since the prediction method relies on the assumption of constant channel parameters. We observe that the performance difference between the scenario with constant parameters and those with varying parameters increases with increasing SNR and that increasing the rate of change, $\beta$, of the parameters increases the NMSE.

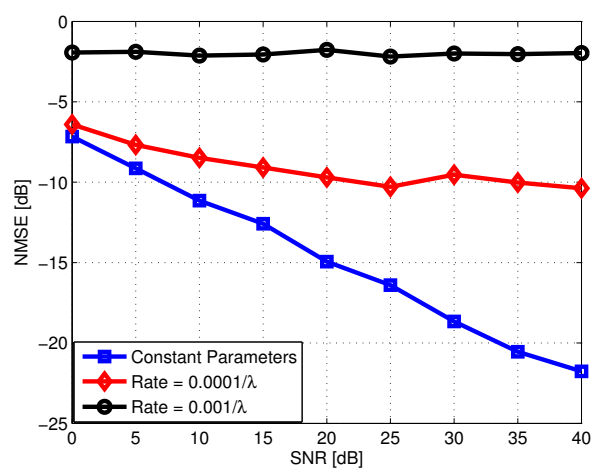

Figure 9.2: Effects of parameter variation on the NMSE.

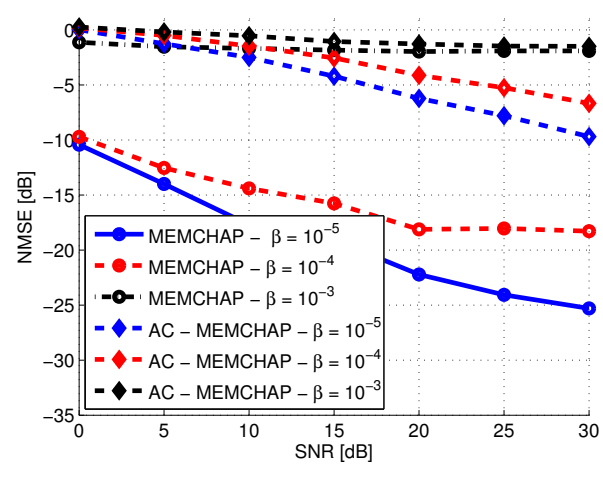

Figure 9.3: NMSE versus SNR. 
A potential method to reduce the performance degradation is to use the recursive covariance matrix estimate given in (3.50), where the spatiotemporal correlation is updated at every time instant as

$$
\hat{\mathbf{R}}_{\mathrm{gg}}(k+1)=\zeta \hat{\mathbf{R}}_{\mathrm{gg}}(k)+(1-\zeta) \hat{\mathbf{g}}(k+1) \hat{\mathbf{g}}(k+1)^{H}
$$

where $\zeta$ is the forgetting factor and $\hat{\mathbf{h}}(k)$ is the $N M K_{c} \times 1$ vector of channel observations at the $k$ th instant. $K_{c}\left(2 \leq K_{c}<K\right)$ corresponds to the small number of samples over which the temporal variation can be neglected. In Fig. 9.3, we compare the performance of the DOD/DOA-MEMCHAP with the covariance matrix estimated using (9.7) with $\zeta=0.4$ and (4.93) for a four path channel. For clarity, we refer to the method using the covariance matrix estimate in (9.7) as Adaptive covariance (AC)-MEMCHAP. In both cases, the variation of the parameters is modeled using the linear advancement model with $\beta_{\theta}=\left\{10^{-3}, 10^{-4}, 10^{-5}\right\}$. We observe that the method utilizing the static covariance matrix estimate in (4.93) outperforms that using (9.7) for variation rates of $10^{-5}$ and $10^{-4}$. However, both methods show similar poor performance for the faster rate of $10^{-3}$. A plausible explanation for the performance gain with the static covariance estimate is that the Hankel matrix formulation in (4.17) allows for the re-use of data at all time instants for both array and averaging gains. This results in more accurate covariance estimate and hence, better parameter estimation prediction. It should be noted that the choice of the forgetting factor $\zeta$ affects the performance of the AC-MEMCHAP. It may therefore be useful to develop methods for finding optimal values of $\zeta$. This is however, outside the scope of this chapter.

\subsubsection{Adaptive MEMCHAP}

In 9.2.1, we illustrated the effects of varying channel parameters on the performance of MEMCHAP and made comparison with a modified approach termed, AC-MEMCHAP, which estimates the covariance matrix adaptively using (9.7). This section derives an adaptive prediction method based on the PAST subspace tracker in Section 3.3.1. The idea is to jointly track 
the parameters and use the parameter evolution to perform prediction. For simplicity we retain the assumption of quasi-stationary number of paths and complex weights and derive an adaptive multidimensional ESPRIT method for tracking the channel parameters. We also assume that the number of paths are known. A summary of the steps in the adaptive MEMCHAP are as follows.

- Adaptive Joint parameter estimation: Given the $K$ noisy CSI estimates, the parameter set $\left\{\mu_{p}^{\mathrm{r}}(k), \mu_{p}^{\mathrm{r}}(k), \nu_{p}(k)\right\}_{p=1}^{P}$ are estimated for $k=$ $1,2, \cdots, K$ in this step.

- Complex amplitude estimation: Using the estimated channel parameters, the complex amplitudes are estimated via a least square approach.

- Parameter extrapolation: This stage involves evaluating future values of the structural parameters using the past estimates.

- CSI prediction: As in the static approach, this stage involves extrapolation of the CSI based on the parameter estimates.

\section{Semi-Adaptive Joint Parameter Estimation}

Let $\hat{\mathbf{h}}(k)$ be the vectorized form of $\hat{\mathbf{H}}(k)$. We assume that the parameters can be assumed constant over every $K_{c}$ instants and define

$$
\hat{\mathbf{d}}(i)=\left[\begin{array}{c}
\hat{\mathbf{h}}\left((i-1) K_{c}+1\right) \\
\hat{\mathbf{h}}\left((i-1) K_{c}+2\right) \\
\vdots \\
\hat{\mathbf{h}}\left(i K_{c}\right)
\end{array}\right] ; i=1, \cdots, I
$$

where $i$ is the new time instant at which parameters are to be estimated and $I=K / K_{c}$. The $I$ data vectors correspond to vectorized version of $I$ groups of $K_{c}$ channel samples. The grouping combines the temporal and spatial channel structure into one dimension, so that 3D parameter estimation can 
be performed. As presented in Section 4.4.4, the central part of the 3D ESPRIT parameter estimation is the solution of invariance equations, thus

$$
\begin{aligned}
& \hat{\boldsymbol{\Phi}}_{\mathrm{d}}=\left(\mathbf{J}_{\mathrm{d} 2} \hat{\mathbf{E}}_{\mathrm{s}}\right)^{\dagger} \mathbf{J}_{\mathrm{d} 1} \hat{\mathbf{E}}_{\mathrm{s}} \\
& \hat{\boldsymbol{\Phi}}_{\mathrm{r}}=\left(\mathbf{J}_{\mathrm{r} 2} \hat{\mathbf{E}}_{\mathrm{s}}\right)^{\dagger} \mathbf{J}_{\mathrm{r} 1} \hat{\mathbf{E}}_{\mathrm{s}} \\
& \hat{\boldsymbol{\Phi}}_{\mathrm{t}}=\left(\mathbf{J}_{\mathrm{t} 2} \hat{\mathbf{E}}_{\mathrm{s}}\right)^{\dagger} \mathbf{J}_{\mathrm{t} 1} \hat{\mathbf{E}}_{\mathrm{s}}
\end{aligned}
$$

where $(\cdot)^{\dagger}$ denotes the pseudo-inverse of the associated matrix, $\mathbf{E}_{\mathrm{s}}$ contains the eigenvectors of the covariance matrix corresponding to the signal subspace and $\mathbf{J}_{x i} ; i=1,2$ are the selection matrices defined in (4.96). The PAST algorithm presented in Section 3.3.1 can be applied to track the variations $\mathbf{E}_{\mathbf{s}}(i)$, thereby eliminating the need for repeated EVD. Let $V_{\mathrm{x} \ell}(i)=$ $\mathbf{J}_{\mathbf{x} \ell} \hat{\mathbf{E}}_{\mathbf{s}}(i) ; \ell=1,2$, the semi-adaptive 3D ESPRIT can therefore be expressed as

$$
\hat{\boldsymbol{\Phi}}_{\mathrm{x}}(i)=V_{\mathrm{x} 1}^{\dagger}(i) V_{\mathrm{x} 1}(i)
$$

Again the MEVD can be applied to (9.12) to obtain automatically paired estimates. Denoting

$$
\begin{aligned}
\hat{\boldsymbol{\Phi}}(i) & =\hat{\boldsymbol{\Phi}}_{\mathrm{r}}(i)+\hat{\boldsymbol{\Phi}}_{\mathrm{t}}(i)+\hat{\boldsymbol{\Phi}}_{\mathrm{d}}(i) \\
& =\mathbf{T}^{-1} \boldsymbol{\Lambda} \mathbf{T},
\end{aligned}
$$

the parameters are obtained as

$$
\begin{aligned}
\hat{\boldsymbol{\mu}}^{\mathrm{r}}(i) & =\arg \left[\operatorname{diag}\left\{\mathbf{T} \hat{\boldsymbol{\Phi}}_{\mathrm{r}}(i) \mathbf{T}^{-1}\right\}\right] \\
\hat{\boldsymbol{\mu}}^{\mathrm{t}}(i) & =\arg \left[\operatorname{diag}\left\{\mathbf{T} \hat{\boldsymbol{\Phi}}_{\mathrm{t}}(i) \mathbf{T}^{-1}\right\}\right] \\
\hat{\boldsymbol{\nu}}(i) & =\arg \left[\operatorname{diag}\left\{\mathbf{T} \hat{\boldsymbol{\Phi}}_{\mathrm{d}}(i) \mathbf{T}^{-1}\right\}\right]
\end{aligned}
$$

The performance of the semi-adaptive joint parameter estimation and tracking is illustrated in Figs. 9.4, where we plot the true and estimated parameters. We consider a $2 \times 2$ narrowband MIMO channel with $P=2$ paths and a mobile velocity $v=50 \mathrm{~km} / \mathrm{h}$ at $\mathrm{SNR}=10 \mathrm{~dB}$. We observe that the PAST based iterative estimation yields reasonable parameter tracking accuracy for all dimensions. 


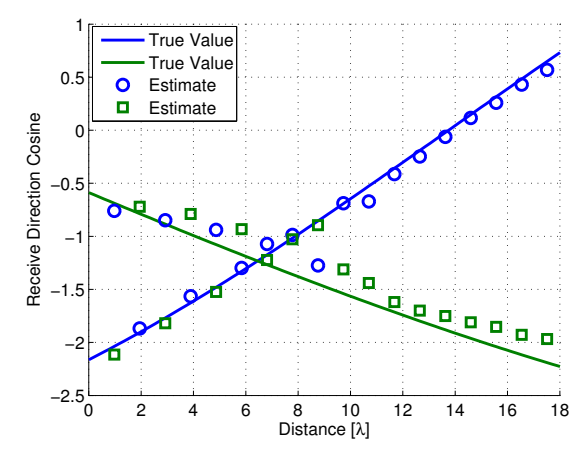

(a) AOA

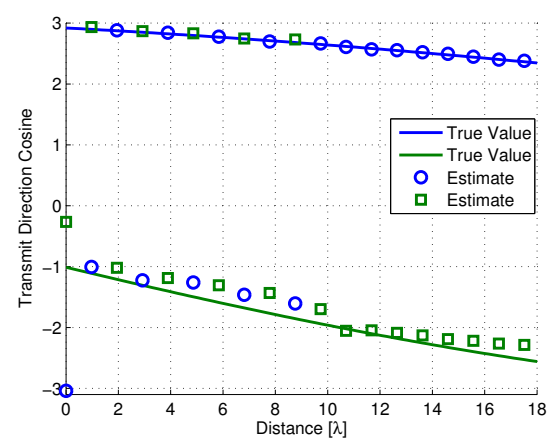

(b) AOD

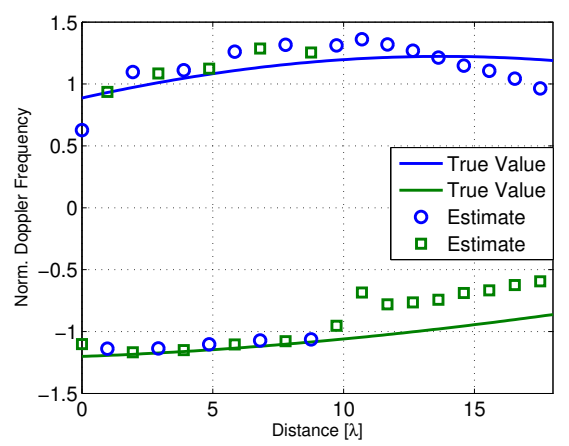

(c) Doppler Shift

Figure 9.4: Joint tracking of AOA, AOD and Doppler shifts at $\mathrm{SNR}=10 \mathrm{~dB}$

As in (9.12), this approach requires explicit computation of the matrices $\hat{\boldsymbol{\Phi}}_{\mathrm{x}}(i)$ at every time instant via a matrix inversion. As shown in [33] for one dimensional estimation, the computation can be eliminated by updating $\hat{\boldsymbol{\Phi}}_{\mathrm{x}}(i)$ recursively. A 3D extension of the adaptive ESPRIT method is presented in the next section.

\section{Adaptive Joint Parameter Estimation}

Consider the PAST subspace tracker in Algorithm 1 on page 54. The signal eigenvector $\mathbf{E}_{\mathbf{s}}(i)$ is obtained via a rank-1 update, thus

$$
\mathbf{E}_{\mathbf{s}}(i)=\mathbf{E}_{\mathbf{s}}(i-1)+\mathbf{e}(i) \mathbf{g}(i)^{H}
$$


Analogously, the update of the matrices in 9.12 can be expressed as

$$
\begin{aligned}
V_{\mathrm{r} 1}(i) & =V_{\mathrm{r} 1}(i-1)+\mathbf{e}_{\mathrm{r} 1}(i) \mathbf{g}(i)^{H} & V_{\mathrm{r} 2}(i) & =V_{\mathrm{r} 2}(i-1)+\mathbf{e}_{\mathrm{r} 2}(i) \mathbf{g}(i)^{H} \\
V_{\mathrm{t} 1}(i) & =V_{\mathrm{t} 1}(i-1)+\mathbf{e}_{\mathrm{t} 1}(i) \mathbf{g}(i)^{H} & V_{\mathrm{t} 2}(i) & =V_{\mathrm{t} 2}(i-1)+\mathbf{e}_{\mathrm{t} 2}(i) \mathbf{g}(i)^{H} \\
V_{\mathrm{d} 1}(i) & =V_{\mathrm{d} 1}(i-1)+\mathbf{e}_{\mathrm{d} 1}(i) \mathbf{g}(i)^{H} & V_{\mathrm{d} 2}(i) & =V_{\mathrm{d} 2}(i-1)+\mathbf{e}_{\mathrm{d} 2}(i) \mathbf{g}(i)^{H}
\end{aligned}
$$

Our aim is to derive expression for recursively updating $\boldsymbol{\Phi}_{\mathrm{r}}, \boldsymbol{\Phi}_{\mathrm{t}}$ and $\boldsymbol{\Phi}_{\mathrm{d}}$. Consider the pseudo-inverse of $V_{\mathrm{r} 1}(i)$ defined as

$$
V_{\mathrm{r} 1}^{\dagger}(i)=\left(V_{\mathrm{r} 1}(i)^{H} V_{\mathrm{r} 1}(i)\right)^{-1} V_{\mathrm{r} 1}(i)^{H}
$$

Let

$$
\mathbf{A}_{\mathrm{r} 1}(i)=V_{\mathrm{r} 1}(i)^{H} V_{\mathrm{r} 1}(i)
$$

and define

$$
\mathbf{B}_{\mathrm{r} 1}(i)=\mathbf{A}_{\mathrm{r} 1}(i)^{-1}
$$

such that (9.17) becomes

$$
V_{\mathrm{r} 1}^{\dagger}(i)=\mathbf{B}_{\mathrm{r} 1}(i) V_{\mathrm{r} 1}(i)^{H}
$$

Substituting the corresponding update equation (9.16) into (9.18) yields

$$
\begin{gathered}
\mathbf{A}_{\mathrm{r} 1}(i)=\left(V_{\mathrm{r} 1}(i-1)+\mathbf{e}_{\mathrm{r} 1}(i) \mathbf{g}(i)^{H}\right)^{H}\left(V_{\mathrm{r} 1}(i-1)+\mathbf{e}_{\mathrm{r} 1}(i) \mathbf{g}(i)^{H}\right) \\
=V_{\mathrm{r} 1}(i-1)^{H} V_{\mathrm{r} 1}(i-1)+V_{\mathrm{r} 1}(i-1)^{H} \mathbf{e}_{\mathrm{r} 1}(i) \mathbf{g}(i)^{H} \\
\quad+\mathbf{e}_{\mathrm{r} 1}(i)^{H} \mathbf{g}(i) V_{\mathrm{r} 1}(i-1)+\left(\left\|\mathbf{e}_{\mathrm{r} 1}(i) \mathbf{g}(i)\right\|^{2}\right)
\end{gathered}
$$

Defining

$$
\begin{gathered}
\mathbf{C}_{\mathrm{r} 1}(i)=\left[V_{\mathrm{r} 1}(i-1)^{H} \mathbf{e}_{\mathrm{r} 1}(i)\right. \\
\mathbf{g}(i)] \\
\mathbf{D}_{\mathrm{r} 1}(i)=\left[\begin{array}{cc}
0 & 1 \\
1 & \left\|\mathbf{e}_{\mathrm{r} 1}(i)\right\|^{2}
\end{array}\right]
\end{gathered}
$$

and using (9.18), (9.21) can be written as

$$
\mathbf{A}_{\mathrm{r} 1}(i)=\mathbf{A}_{\mathrm{r} 1}(i-1)+\mathbf{C}_{\mathrm{r} 1}(i) \mathbf{D}_{\mathrm{r} 1}(i) \mathbf{C}_{\mathrm{r} 1}(i)^{H}
$$


Now substituting (9.24) into (9.19) yields

$$
\mathbf{B}_{\mathrm{r} 1}(i)=\left(\mathbf{A}_{\mathrm{r} 1}(i-1)+\mathbf{C}_{\mathrm{r} 1}(i) \mathbf{D}_{\mathrm{r} 1}(i) \mathbf{C}_{\mathrm{r} 1}(i)^{H}\right)^{-1}
$$

Assuming that $\mathbf{A}_{\mathrm{r} 1}(i-1), \mathbf{C}_{\mathrm{r} 1}(i)$ and $\mathbf{D}_{\mathrm{r} 1}(i)$ are non-singular matrices, the matrix inversion in (9.25) can be expressed as [33]

$$
\begin{aligned}
\mathbf{B}_{\mathrm{r} 1}(i)= & \mathbf{A}_{\mathrm{r} 1}(i-1)^{-1}-\mathbf{A}_{\mathrm{r} 1}(i-1)^{-1} \mathbf{C}_{\mathrm{r} 1}(i)\left(\mathbf{D}_{\mathrm{r} 1}(i)^{-1}+\mathbf{C}_{\mathrm{r} 1}(i)^{H} \mathbf{A}_{\mathrm{r} 1}(i-1)^{-1} \mathbf{C}_{\mathrm{r} 1}(i)\right)^{-1} \\
& \times \mathbf{C}_{\mathrm{r} 1}(i)^{H} \mathbf{A}_{\mathrm{r} 1}(i-1)^{-1} \\
= & \mathbf{B}_{\mathrm{r} 1}(i-1)-\mathbf{B}_{\mathrm{r} 1}(i-1) \mathbf{C}_{\mathrm{r} 1}(i)\left(\mathbf{D}_{\mathrm{r} 1}(i)^{-1}+\mathbf{C}_{\mathrm{r} 1}(i)^{H} \mathbf{B}_{\mathrm{r} 1}(i-1) \mathbf{C}_{\mathrm{r} 1}(i)\right)^{-1} \\
& \times \mathbf{C}_{\mathrm{r} 1}(i)^{H} \mathbf{B}_{\mathrm{r} 1}(i-1)
\end{aligned}
$$

Letting

$$
\boldsymbol{\Xi}_{\mathrm{r} 1}(i)=\mathbf{B}_{\mathrm{r} 1}(i-1) \mathbf{C}_{\mathrm{r} 1}(i),
$$

and

$$
\boldsymbol{\Sigma}_{\mathrm{r} 1}(i)=\left(\mathbf{D}_{\mathrm{r} 1}(i)^{-1}+\boldsymbol{\Xi}_{\mathrm{r} 1}(i) \mathbf{C}_{\mathrm{r} 1}(i)\right)^{-1},
$$

(9.26) reduces to

$$
\mathbf{B}_{\mathrm{r} 1}(i)=\mathbf{B}_{\mathrm{r} 1}(i-1)-\boldsymbol{\Xi}_{\mathrm{r} 1}(i) \boldsymbol{\Sigma}_{\mathrm{r} 1}(i) \boldsymbol{\Xi}_{\mathrm{r} 1}(i)^{H}
$$

Substituting (9.29) into (9.20) yields

$$
V_{\mathrm{r} 1}^{\dagger}(i)=\left(\mathbf{B}_{\mathrm{r} 1}(i-1)-\boldsymbol{\Xi}_{\mathrm{r} 1}(i) \boldsymbol{\Sigma}_{\mathrm{r} 1}(i) \boldsymbol{\Xi}_{\mathrm{r} 1}(i)^{H}\right)\left(V_{\mathrm{r} 1}(i-1)+\mathbf{e}_{\mathrm{r} 1}(i) \mathbf{g}(i)^{H}\right)
$$

After straightforward mathematical simplifications, (9.30) becomes $^{2}$

$$
V_{\mathrm{r} 1}^{\dagger}(i)=V_{\mathrm{r} 1}^{\dagger}(i-1)+\Upsilon(i) \boldsymbol{\Omega}(i)^{H}
$$

where

$$
\Upsilon(i)=\Xi_{\mathrm{r} 1}(i) \boldsymbol{\Sigma}_{\mathrm{r} 1}(i)
$$

and

$$
\boldsymbol{\Omega}(i)=\left[\begin{array}{ll}
\mathbf{e}_{\mathrm{r} 1}(i) & \mathbf{0}
\end{array}\right]-V_{\mathrm{r} 1}(i-1) \boldsymbol{\Xi}_{\mathrm{r} 1}(i)
$$

\footnotetext{
${ }^{2} \mathrm{~A}$ similar recursive update expression have been presented in [33] for the onedimensional adaptive ESPRIT.
} 
Substituting (9.31) and the update equation for $V_{\mathrm{r} 2}(i)$ in (9.16) into (9.12) gives

$$
\begin{aligned}
\hat{\boldsymbol{\Phi}}_{\mathrm{r}}(i)= & \left(V_{\mathrm{r} 1}^{\dagger}(i-1)+\mathbf{\Upsilon}(i) \boldsymbol{\Omega}(i)^{H}\right)\left(V_{\mathrm{r} 2}(i-1)+\mathbf{e}_{\mathrm{r} 2}(i) \mathbf{g}(i)^{H}\right) \\
= & V_{\mathrm{r} 1}^{\dagger}(i-1) V_{\mathrm{r} 2}(i-1)+V_{\mathrm{r} 1}^{\dagger}(i-1) \mathbf{e}_{\mathrm{r} 2}(i) \mathbf{g}(i)^{H} \\
& \quad+\boldsymbol{\Upsilon}(i) \boldsymbol{\Omega}(i)^{H} V_{\mathrm{r} 2}(i-1)+\mathbf{\Upsilon}(i) \boldsymbol{\Omega}(i)^{H} \mathbf{e}_{\mathrm{r} 2}(i) \mathbf{g}(i)^{H} \\
= & \hat{\boldsymbol{\Phi}}_{\mathrm{r}}(i-1)+V_{\mathrm{r} 1}^{\dagger}(i-1) \mathbf{e}_{\mathrm{r} 2}(i) \mathbf{g}(i)^{H}+\boldsymbol{\Upsilon}(i) \boldsymbol{\Omega}(i)^{H} V_{\mathrm{r} 2}(i)
\end{aligned}
$$

Defining

$$
\boldsymbol{\Gamma}(i)=\left[\begin{array}{ll}
V_{\mathrm{r} 1}^{\dagger}(i-1) \mathbf{e}_{\mathrm{r} 2}(i) & \mathbf{\Upsilon}(i)
\end{array}\right]
$$

and

$$
\Pi(i)=\left[\mathbf{g}(i) \quad V_{\mathrm{r} 2}(i)\right]
$$

(9.34) can be written as

$$
\hat{\boldsymbol{\Phi}}_{\mathrm{r}}(i)=\hat{\boldsymbol{\Phi}}_{\mathrm{r}}(i-1)+\boldsymbol{\Gamma}(i) \boldsymbol{\Pi}(i)^{H}
$$

Expressions for updating $\hat{\boldsymbol{\Phi}}_{\mathrm{t}}(i)$ and $\hat{\boldsymbol{\Phi}}_{\mathrm{d}}(i)$ are obtained following a similar procedure. Again, we use (9.13) and (9.14) to extract the channel parameters.

\section{Complex Amplitude Estimation}

Since the complex amplitudes are assumed to be constant over the entire region of interest ${ }^{3}$, the first entry of (9.2) can be expressed as

$$
\hat{h}_{11}(i)=\sum_{p=1}^{P} \alpha_{p} e^{j(i-1) \nu_{p}(i)}+w(i) ; i=1, \cdots, I
$$

Collecting the $I$ equations in (9.38) gives

$$
\left[\begin{array}{c}
\hat{h}_{11}(1) \\
\hat{h}_{11}(2) \\
\vdots \\
\hat{h}_{11}(I)
\end{array}\right]=\left[\begin{array}{cccc}
1 & 1 & \cdots & 1 \\
e^{j \nu_{1}(2)} & e^{j \nu_{2}(2)} & \cdots & e^{j \nu_{P}(2)} \\
\vdots & \vdots & \ddots & \vdots \\
e^{j(I-1) \nu_{1}(I)} & e^{j(I-1) \nu_{2}(I)} & \cdots & e^{j(I-1) \nu_{P}(I)}
\end{array}\right]\left[\begin{array}{c}
\alpha_{1} \\
\alpha_{2} \\
\vdots \\
\alpha_{P}
\end{array}\right]+\mathbf{w}
$$

\footnotetext{
${ }^{3}$ This assumption is only made here for simplicity reasons. In practice, the complex amplitudes may also exhibit temporal/spatial variations necessitating the need for methods to model their variation and tracking. This could be investigated in our future works.
} 
Table 9.1: Simulation Parameters

\begin{tabular}{ll}
\hline Parameter & value \\
\hline Carrier Frequency & $2.1 \mathrm{GHz}$ \\
Mobile Velocity & $50 \mathrm{kp} / \mathrm{h}$ \\
Tx $/$ Rx Antenna Conf. & $\mathrm{ULA} @ 1 / 2 \lambda$ spacing \\
Sampling Rate & $10 / \lambda$ \\
Variation Rate $(\beta)$ & $10^{-3}$ \\
Training Length & 100 \\
\hline
\end{tabular}

which can be compactly written as

$$
\hat{\mathbf{h}}_{11}=\mathbf{G} \boldsymbol{\alpha}+\mathbf{w}
$$

By minimizing the MSE in (9.40), we obtain

$$
\hat{\boldsymbol{\alpha}}=\left(\mathbf{G}^{H} \mathbf{G}\right)^{-1} \mathbf{G}^{H} \hat{\mathbf{h}}_{11}
$$

\section{CSI Prediction}

Having estimated the time-varying channel parameters over the observation segment, the predicted CSI at a desired instant can be obtained by first extrapolating the parameters and then substituting into the model. Extrapolation of the channel parameters can be achieved by using either a linear or polynomial regression methods. However, there is an inherent problem of parameter association over time, making it difficult to apply any of the prediction methods. An alternative approach is to use the most current parameter estimates for evaluating future values of the CSI, thus

$$
\tilde{\mathbf{H}}(k)=\sum_{p=1}^{P} \hat{\alpha}_{p} \mathbf{a}_{\mathbf{r}}\left(\mu_{p}^{\mathrm{r}}(K)\right) \mathbf{a}_{\mathrm{t}}^{T}\left(\mu_{p}^{\mathrm{t}}(K)\right) e^{j k \nu_{p}(K)}
$$

for $k=K+1, K+2, \cdots$. 


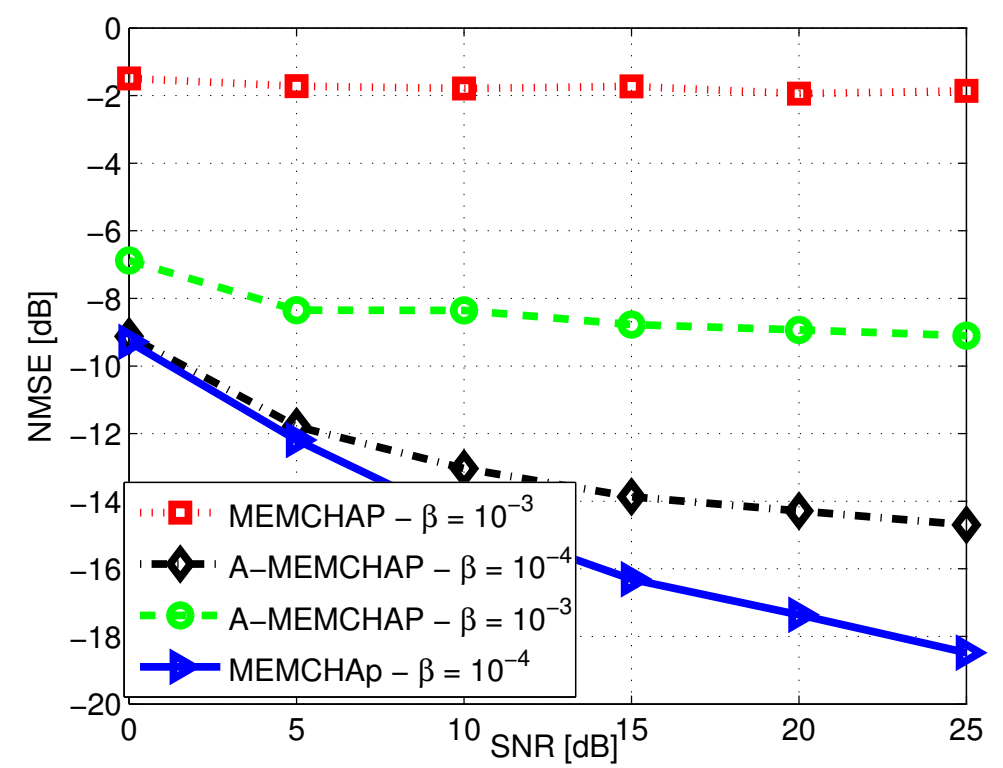

Figure 9.5: NMSE of adaptive method at different variation rates.

\subsection{Simulation and Results}

In this section, we evaluate the performance of the adaptive prediction method. We consider a $2 \times 2$ narrowband channel with parameters in Table 9.1. Fig. 9.5 presents the prediction NMSE as a function of SNR at a prediction horizon of $0.1 \lambda$. We observe that, with parameter variation rate of $10^{-3}$, the PAST based adaptive method decreases the performance NMSE of DOD/DOAMEMCHAP scheme by approximately $6 \mathrm{~dB}$ at all SNR values considered. However, both methods yield similar NMSE at low SNR for a slower rate of $10^{-4}$ with the DOD/DOA-MEMCHAP performing better at high SNR values. An explanation for this is that, while the adaptive method is able to overcome the degradation resulting from parameter variation the static method make better use of the measurements. 


\subsection{Summary}

In this chapter, we have investigated methods for jointly tracking the structural parameters of a narrowband MIMO channels based on a multidimensional extension of the adaptive ESPRIT scheme. The parameter tracking is achieved via a PAST subspace tracker. Simulation results shows that while the DOD/DOA-MEMCHAP approach in Chapter 4 outperforms the adaptive method for slowly varying channel parameters, the adaptive scheme offer improved prediction NMSE performance with increased variation of the channel parameters. Incorporating variation of complex amplitudes and evolution of the number of scatterers into the adaptive scheme could be considered in future research. 


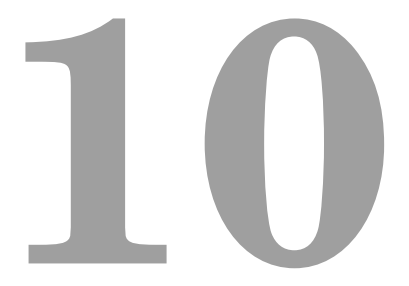

\section{Conclusions and Future Research}

\subsection{Summary of Contributions}

$\prod \begin{gathered}\text { HIS thesis investigated the prediction of mobile MIMO channels to enable } \\ \text { adaptive transmission and limited feedback techniques. Our focus is on }\end{gathered}$ the utilization of temporal, spatial and frequency structure of the channel to improve parameter estimation and prediction accuracy.

We began our investigations with the exploration of bandlimited extrapolation methods (see Appendix B) for narrowband mobile MIMO wireless channels. A discrete solution of the $2 \mathrm{D}$ prolate spheroidal wave functions (PSWF) was derived based on the 1D solution in [98]. Based on the bandlimited properties of wireless channels, we evaluated the performance of both iterative and non-iterative bandlimited extrapolation schemes for MIMO channel predictions. We further derived schemes utilizing the discrete prolate spheroidal sequences (DPSS) for predicting both narrowband and wideband MIMO channels. We observed that while the DPSS based methods offer reasonable performance particular for short range prediction, they suffer from 
a number of limitations such as sensitivity to noise, requirement for oversampled training data, and most importantly, non-utilization of the spatial information offered by the presence of multiple antenna elements. Compared to the application of the one-dimensional ESPRIT based prediction scheme in [24], we observed that while the DPSS performed better in the training segment, the prediction performance of the ESPRIT scheme are generally better and sensitivity to sampling rate is much lower. These reasons motivated a shift of our research focus to parametric radio channel based approaches.

Several schemes based on spatial channel modeling and multidimensional ESPRIT are proposed for pilot based narrowband mobile MIMO systems in Chapter 4. Using the double directional MIMO channel model, we derive variations of prediction models by eliminating dependence on transmit and/or receive array geometry. The prediction models are termed DOD/DOA, TSSM, RSSM, and MSSM and the prediction methods are referred to as MEMCHAP. The acronym for the model are used as prefixes to differentiate the methods. Our investigations showed that utilizing the spatial structure of the MIMO channel results in improved parameter estimation and CSI prediction accuracy, with the method utilizing both transmit and receive spatial structure (DOD/DOA-MEMCHAP) outperforming those with only receive (TSSM-MEMCHAP), only transmit (RSSM-MEMCHAP) and no spatial information (MSSM-MEMCHAP). In order to investigate the effects of the elevation spectrum on CSI prediction, we extended the parametric prediction concept to a $3 \mathrm{D}$ propagation scenario with UPA at the BS and a ULA at the MS.

In Chapter 5, we extended the prediction concept to pilot based wideband mobile MIMO channels. As in the narrowband case, we refer to the wideband methods as WIMEMCHAP and used the acronyms for the models as prefixes. Motivated by the cluster based modeling approach in recent standardized models, where rays are grouped into clusters based on their delay information, we proposed a cluster based method termed CBM-WIMEMCHAP. Unlike the non-cluster based methods, the CBM-WIMEMCHAP involves a 
two-stage parameter estimation, where the cluster delays are estimated in the first stage using the frequency statistics of the channel followed by a multidimensional estimation of the structural parameters for each cluster using the spatial and temporal information. Experiments using the WINNER II SCM model revealed that the CBM-MEMCHAP offer better prediction when compared to the non-cluster based methods.

As a result of the difference in modeling, parametric prediction schemes are presented for mobile-to-mobile and polarized MIMO channels in Chapters 6 and 7, respectively. A simple method for estimating the transmitter and receiver velocity in M-to-M channels is also proposed. We observed that polarization diversity improves parameter estimation and CSI prediction performance by virtue of data averaging from multiple polarization dimensions. The effect of antenna orientations (i.e., slant angles) on channel prediction was also studied and we observe that systems with $0, \pi / 2$ slant angles offer improved performance compared to those with $\pm \pi / 4$.

In Chapter 8, we derived bound on the prediction of mobile MIMO wireless channel in 2D and 3D propagation environments using a vector formulation of the CRB for functions of parameters. Motivated by the need for readily interpretable expressions for the FIM and prediction error bound, we derived closed form expressions in the asymptotic limits of large number of training samples and/or antenna elements. We showed via simulations that the asymptotic expressions provide close approximation to the error bound while eliminating the need for repeated computation.

Lastly, we studied the effect of temporal variation of channel parameters on the performance of the proposed prediction methods in Chapter 9. We observed that increased variation of the channel parameters degrades the performance of the MEMCHAP scheme. An adaptive multidimensional ESPRIT based predictor named AMECHAP is proposed based on the PAST subspace tracker, where the parameter estimation stage involve jointly tracking the evolution of the channel parameters. Simulation results showed that the adaptive approach offered improved performance for fast parameter vari- 
ation rate.

\subsection{Conclusions}

In this thesis, we have developed several approaches for real time prediction of narrowband mobile MIMO wireless channels. Based on parametric modeling of the double directional channel, we derived different versions of the model upon which our prediction methods are based. We proposed an adaptation of multidimensional ESPRIT for jointly extracting the parameters of the models and extrapolation is achieved using the estimated parameters. We have also studied the effects of using the spatial structure of the MIMO channel on channel parameter estimation and CSI prediction. Our results indicate that methods utilizing the spatial information for array gain outperform the classical SISO based schemes, in which the MIMO channel entries are treated as independent SISO links. We also observed that utilizing both the transmit and receive spatial channel structure as in the DOD/DOA methods results in better parameter estimation and CSI prediction accuracy than those with only transmit (i.e., RSSM) and only receive (i.e., TSSM) spatial information.

The proposed prediction methods have also been extended to other scenarios including wide-band systems, mobile-to-mobile transmission and systems with polarized antenna arrays. A simple method for estimating the velocity of the transmitter and receiver in mobile-to-mobile systems using the channel parameters is also presented. We have also investigated the effects of antenna orientation on the prediction of polarized MIMO channels using the two common dual polarized antenna configurations, i.e., $\pm \pi / 4$ and $0, \pi / 2$. We observed that the $0, \pi / 2$ antenna configuration offers better prediction performance.

Based on the vector formulation of the CRB for function of parameters, we derived simple closed-form expressions for the prediction error bounds for all the scenarios considered. Our results indicated that the performance of the DOD/DOA-MEMCHAP approaches the bound with increased SNR 
and/or sample length. The derived bounds show that prediction error grows linearly with number of paths and noise variance and quadratically with the prediction horizon in both time and frequency. The bounds also show an inverse relationship between the error bound and number of transmit and receive antenna elements.

In order to relax the parameter stationarity assumption in our prediction methods, we have also developed a method for tracking the slow variation of the channel parameters based on the PAST subspace tracker. The adaptive approach was shown via simulations to outperform the DOD/DOAMEMCHAP method when the channel parameters are allowed to vary over time. Relaxation of the stationarity assumption makes real time prediction of wireless channel more feasible in practice.

\subsection{Future Research}

This thesis has made significant contributions to mobile MIMO channel prediction. Since research works often lead to the identification of areas that require further exploration, we highlight below some research topics based on our findings.

- Complexity reduction: Since the prediction methods are based on multidimensional ESPRIT, a straightforward approach to reduce the computation load would be transforming the complex-valued processing in the parameter estimation stage into real-valued computations. This can be achieved by using multidimensional extension of Unitary-ESPRIT [73], which has been shown to offer improved parameter estimation performance at a lower complexity compared to the ESPRIT used in our methods. Investigation into data transformation approaches which satisfy the centro-symmetric data requirement in addition to translational invariance will be addressed in our ongoing research.

- Performance evaluation using measured data: Extensive performance 
evaluation of the prediction schemes presented in this thesis using measured channel data is an interesting topic for further research. Applicability and/or extensions to other common array geometries such as the circular and spherical arrays may also be useful.

- Developing models for parameter variation using measured channels: Another interesting research topic may involve developing models to capture the variation of multipath parameters based on channel measurements.

- Effects of array imperfections/antenna coupling on the algorithm performance: It may also be interesting to investigate the effects of antenna array imperfections and mutual coupling on the performance of the proposed methods.

- Parameter association: As mentioned in Chapter 9, a potential method to improve prediction when the channel parameters are time-varying is to extrapolate the parameters before applying them for CSI prediction. An interesting problem for further research is the development of methods for associating the estimated parameters over time.

- Effects of synchronization errors: It may also be very useful to relax the perfect synchronization assumptions made in this thesis and investigate the effects of synchronization errors on the prediction performance.

- Useful of Erroneous Channel State Information at the Transmitter (CSIT): The main motivation for channel state prediction is to improve the accuracy of the CSI at both ends of a wireless link. In a recent study on the usefulness of bad CSI at the transmiter [123], the authors showed that a degree of freedom greater than unity is achievable in a MIMO broadcast channel even when the CSI is completely outdated. Since, the analysis in the paper only considered multiuser MISO broadcast channels and relied on a number of assumptions, it 
may be worthwhile to investigate the validity of these claims and applicability to other MIMO configurations such as the multiple access channels and point-to-point transmission.

- Application: Another interesting area for further research is to incorporate the schemes proposed in this thesis into MIMO communication systems requiring transmit side CSI such as codebook based limited feedback schemes, scheduling and coordinated transmission. The overall system performance and/or performance improvements with predicted CSI may then be investigated using other performance metrics. Our ongoing research is evaluating the effects of channel aging on linear precoding schemes including Zero-Forcing (ZF), Signal-to-Leakage Noise Ratio (SLNR), Regularized Zero-Forcing (RZF) and Block Diagonalization (BD) for the down-link of multiuser MIMO systems. Our prediction schemes will be incorporated into these precoders and the overall system performance will be evaluated using metrics such as sum rate and outage probability. A summary of our preliminary investigations and the proposed study is presented in Appendix C. 


\section{A \\ Contributions to Bandlimited Signal Extrapolation}

\section{A.1 Introduction}

$\mathrm{B}$ ANDLIMITED signal extrapolation (BSE) is a common problem in many theoretical and applied fields such as spectral estimation, image restoration, Fourier analysis and wireless communications and has been the focus of several research works (see e.g., $[39,66,89,145,160,180])$. In this chapter, we investigate the prediction of mobile MIMO channels using bandlimited extrapolation techiques. After a brief review of bandlimited signal extrapolation in Section A.2, we present an introduction to the Prolate Spheroidal Wave Function (PSWF) along with a derivation of a discrete solution of 2D PSWF in Section A.3. Methods for the prediction of narrowband and wideband MIMO channels using DPSS are then described in Section A.4. 


\section{A.2 Bandlimited Signal Extrapolation (BSE)}

A function $f(t) \in L^{2}(\mathbf{R})$ is said to be $\sigma$-bandlimited if

$$
\mathfrak{F}\{f(t)\}=0 \quad \forall \omega \in \mathbf{R}
$$

where $\mathfrak{F}$ denotes the Fourier transformation defined as

$$
\mathfrak{F}\{f(t)\}=\int_{-\infty}^{\infty} f(t) e^{j \omega t} \mathrm{~d} t
$$

with the inversion formula given by

$$
\mathfrak{F}^{-1}\{f(\omega)\}=\frac{1}{2 \pi} \int_{-\sigma}^{\sigma} f(\omega) e^{-j \omega t} \mathrm{~d} \omega
$$

Assuming that $f(t)$ is known over the interval $[-T, T]$, the problem of bandlimited extrapolation is to find the signal outside the known region. Several iterative and non-iterative methods have been proposed for BSE. These methods are generally classified into: Minimum energy extrapolation, TimeBandwidth dimension based extrapolation and energy concentration extrapolation. A review of selected iterative and non-iterative methods for the extrapolation of bandlimited signals is presented in this section.

\section{A.2.1 Iterative Method}

Similar to the Gerchberg's iterative algorithm for improving data resolution through error energy reduction [66], Papoulis presented an iterative extrapolation method in [145] which converges to the actual bandlimited signal if the known data is noise-free. The Papoulis approach can be represented in equation form as

$$
f^{[k+1]}=P_{T} f(t)+\left(I-P_{T}\right) \mathfrak{F}^{-1}\left\{P_{\sigma} \mathfrak{F}\left\{f^{[k]}\right\}\right\}
$$

for $k=0,1,2, \cdots . P_{T}$ and $P_{\sigma}$ are time-limiting and band-limiting operators defined as

$$
P_{T} f(t)= \begin{cases}f(t) & t \in[-T, T] \\ 0 & t \notin[-T, T]\end{cases}
$$


and

$$
P_{\sigma} f(\omega)= \begin{cases}f(\omega) & \omega \in[-\sigma, \sigma] \\ 0 & t \notin[-\sigma, \sigma]\end{cases}
$$

respectively. Proof of the convergence of the iterative algorithm can be found in [145]. Note that the known segment of the signal for initializing the iteration can be denoted as $f^{[0]}(t)=P_{T} f(t)$.

\section{A.2.2 Extrapolation Matrix Method (EME)}

Motivated by the slow convergence rate of the Papoulis iterative method, an extrapolation matrix method, where the iterative procedure is replaced with a single matrix operation, was proposed in [160]. Using (A.4), the first iteration of the iterative method can be expressed as

$$
f^{[1]}=f^{[0]}+f^{[0]} \mathbf{B X}
$$

where $\mathbf{X}=\operatorname{diag}\{1,1, \cdots, 1,0, \cdots, 0,0, \cdots, 0,1,1\}$ is a diagonal switching matrix that makes the known segment zero and $\mathbf{B}=\mathbf{W}^{-1} \mathbf{G} \mathbf{W} . \mathbf{W}$ is the discrete Fourier transform (DFT) matrix and $\mathbf{G}$ is a matrix of weights. Similarly, the second and third iteration can be shown to be

$$
f^{[2]}=f^{[0]}+f^{[1]} \mathbf{H}=f^{[0]}+f^{[0]} \mathbf{H}+f^{[0]} \mathbf{H}^{2}
$$

and

$$
f^{[3]}=f^{[0]}+f^{[2]} \mathbf{H}=f^{[0]}+f^{[0]} \mathbf{H}+f^{[0]} \mathbf{H}^{2}+f^{[0]} \mathbf{H}^{3}
$$

where $\mathbf{H}=\mathbf{B X}$. It follows from (A.7), (A.8) and (A.9) that

$$
\begin{aligned}
f^{[N]} & =f^{[0]}+f^{[0]} \mathbf{H}+f^{[0]} \mathbf{H}^{2}+f^{[0]} \mathbf{H}^{3}+\cdots+f^{[0]} \mathbf{H}^{N} \\
& =f^{[0]} \sum_{n=0}^{N} \mathbf{H}^{n} \\
& =f^{[0]} \mathbf{E}_{N}
\end{aligned}
$$


where $\mathbf{E}_{N}=\sum_{n=0}^{N} \mathbf{H}^{n}$ is the extrapolation matrix at the $N$ th iteration. For $N=\infty$, the extrapolation matrix for infinite number of iterations was shown to be [160]

$$
\mathbf{E}_{\infty}=(\mathbf{I}-\mathbf{H})^{-1}
$$

\section{A.2.3 Minimum Norm Least Square Extrapolation}

The extrapolation matrix approach is a discretized version of the iterative algorithm for continuous signals. However, its convergence to the original signal is not guaranteed since the analytic property of continuous signals upon which the algorithm is based does not hold for discrete signals. In order to overcome this limitation, a minimum norm least square (MNLS) method is proposed in [89] for converting the continuous extrapolation into discrete form.

\section{A.3 Prolate Spheroidal Wave Function}

In this section, a discussion about prolate spheroidal wave functions along with discrete solution of PSWFs in 2D is presented.

The PSWFs $[178,179]$ popularly known as Slepian functions have been studied extensively as a basis for decomposition and reconstruction of bandlimited signals. The PSWFs $\Psi_{k}(t)^{1}$ with concentration on the interval $[-T, T]$ are the normalized eigenfunctions of the integral equation [179]

$$
\int_{-T}^{T} \Psi_{k}(t) \frac{\sin \sigma(x-t)}{\pi(x-t)} d t=\lambda_{k} \Psi_{k}(x)
$$

where $t$ is the continuous time variable and $k$ represents the order of the eigenfunction. $\lambda_{k}$ is the eigenvalue of the sinc kernel which serves as a measure of how concentrated $\Psi_{k}(t)$ is on the interval $[-T, T]$. In practice, the

\footnotetext{
${ }^{1}$ Note that the PSWF can also be parametrized in terms of the time-bandwidth parameter, defined as $c=\sigma T$.
} 
PSWFs and associated eigenvalues may be computed numerically using [98]

$$
\Psi_{k}(t)=\sqrt{\frac{\lambda_{k} / T}{\int_{-1}^{1}\left(S_{0 n}(t)\right)^{2} d t}} S_{0 n}\left(\frac{t}{T}\right)
$$

and

$$
\lambda_{k}=\frac{2 c R_{0 n}(1)^{2}}{\pi}
$$

where $S_{m, n}(\eta)$ and $R_{m, n}(\eta)$ are the angular and radial solutions of the Helmholtz equation of the first kind, respectively. Some relevant properties of the PSWF and procedures for signal extrapolation using the PSWF are given below.

\section{A.3.1 Properties}

The PSWFs have several fascinating properties, some of which includes:

\section{Invariance to Fourier Transform}

The PSWF $\Psi_{k}(t)$ are invariant to both finite and infinite Fourier transforms which are given by

$$
\int_{-T}^{T} \Psi_{k}(t) e^{j \omega t} d t=j^{k}\left(\frac{2 \pi \lambda_{k} T}{\sigma}\right)^{1 / 2} \Psi_{k}\left(\frac{\omega T}{\sigma}\right)
$$

and

$$
\int_{-\infty}^{\infty} \Psi_{k}(t) e^{j \omega t} d t=j^{k}\left(\frac{2 \pi T}{\sigma}\right)^{1 / 2} \Psi_{k}\left(\frac{\omega T}{\sigma}\right)
$$

respectively. The invariance to Fourier transformation is a result of the bandlimited property of the PSWFs which is measured using the bandwidth parameter $c$.

\section{Dual Orthogonality}

Another interesting property of the PSWF and possibly the most important for signal extrapolation is dual orthogonality. Unlike other basis functions, 
which are only orthogonal over a finite interval, the PSWFs are orthogonal on the finite domain and orthonormal in the infinite domain i.e.,

$$
\begin{aligned}
& \int_{-T}^{T} \Psi_{k}(t) \Psi_{\ell}(t) d t= \begin{cases}\lambda_{k} & \text { for } k=\ell \\
0 & \text { otherwise }\end{cases} \\
& \int_{-\infty}^{\infty} \Psi_{k}(t) \Psi_{\ell}(t) d t= \begin{cases}1 & \text { for } k=\ell \\
0 & \text { otherwise }\end{cases}
\end{aligned}
$$

\section{Bandlimitedness}

The bandlimited property of the PSWFs is measured using the Slepian frequency (also known as time-bandwidth parameter) defined as

$$
c=\sigma T
$$

where $\sigma$ denotes the bandwidth of the PSWFs.

\section{Even/Odd Symmetry}

Depending on the order $k$ of a PSWF, it exhibits either even or odd symmetry. If $k$ is even, the PSWF has even symmetry and vice versa. Detailed discussion and proof of these properties can be found in [178, 179].

\section{A.3.2 Signal Extrapolation Using PSWF}

It follows from (A.18) that any continuous function $f(t)$ can be represented as a weighted summation using this orthonormal basis as

$$
f(t)=\sum_{k=0}^{\infty} \gamma_{k} \Psi_{k}(t)
$$

where $\gamma_{k}$ are scalar weighting factors defined as

$$
\begin{aligned}
\gamma_{k} & =\frac{\int_{-\infty}^{\infty} f(t) \Psi_{k}(t) d t}{\int_{-\infty}^{\infty} \Psi_{k}(t) \Psi_{k}(t) d t} \\
& =\int_{-\infty}^{\infty} f(t) \Psi_{k}(t) d t
\end{aligned}
$$


Using Parseval's theorem, the coefficients $\gamma_{k}$ satisfy

$$
\sum_{k=0}^{\infty} \gamma_{k}^{2}=\int_{-\infty}^{\infty}|f(t)|^{2} d t
$$

The basis expansion in (A.20) therefore converges to the true value of $f(t)$ with convergence in a squared error sense given by [179]

$$
\lim _{K \rightarrow \infty} \int_{-\infty}^{\infty}\left\{f(t)-\sum_{k=0}^{K} \gamma_{k} \Psi_{k}(t)\right\}^{2} d t=0
$$

An approximation for $f(t)$ based on (A.23) is thus

$$
f(t) \approx \sum_{k=0}^{K} \gamma_{k} \Psi_{k}(t)
$$

Note that the approximation in (A.24) is well suited for functions whose energy distribution span an infinite interval. However, most practical extrapolation problems involve functions defined over a finite time domain. Such functions can also be approximated using (A.24) with

$$
\gamma_{k}=\frac{1}{\lambda_{k}} \int_{-T}^{T} f(t) \Psi_{k}(t) d t
$$

Extrapolation of wireless MIMO channels using the concept of bandlimited extrapolation and specifically the PSWF will be addressed in A.4.

\section{A.3.3 2D Prolate Spheroidal Wave Function}

The 2D prolate spheroidal wave functions (PSWF) are the eigenfunctions of the integral equation

$$
\int_{-\gamma}^{\gamma} \int_{-\tau}^{\tau} \Psi(u, v) \frac{\sin (\Phi(x-u))}{\pi(x-u)} \frac{\sin (\varphi(y-v))}{\pi(y-v)} d x d y=\lambda \Psi(x, y)
$$

The techniques used in the literature for the computation of the PSWFs include the asymptotic solutions of Prolate spheroidal differential equations 
(PSDE) and Bouwkamp's method of Legendre series. The computational complexity of these methods limits the applications of the PSWF. In [98], a discrete solution was proposed for 1D PSWF. Using the Whittaker-Shannon sampling theorem [172] in 2D, we propose a discrete solution for the $2 \mathrm{D}$ PSWF.

\section{D Shannon Sampling Theorem}

The Shannon's theorem in 2D implies that the exact reconstruction of a bandlimited function $g(x, y)$ can be achieved from an appropriately spaced array of its samples $g(n, m)=g\left(n \Pi_{x}, m \Pi_{y}\right)$

$$
g(x, y)=\sum_{n=-\infty}^{\infty} \sum_{m=\infty}^{\infty} g(n, m) \operatorname{sinc}\left(\frac{x-n \Pi_{x}}{\Pi_{x}}\right) \operatorname{sinc}\left(\frac{y-m \Pi_{y}}{\Pi_{y}}\right) d x d y
$$

where $\Pi_{x}$ and $\Pi_{y}$ are the sampling intervals in the $x$ and $y$-direction respectively.

\section{Solution of 2D PSWF}

Consider the integral equation in (A.26). The kernel of the equation can be decomposed using the sampling theorem (A.27) as

$$
\begin{aligned}
K(x, y) & \triangleq \operatorname{sinc}(\Phi(x-u)) \operatorname{sinc}(\varphi(y-v)) \\
& =\sum_{i=-\infty}^{\infty} \sum_{j=\infty}^{\infty} \operatorname{sinc}(\Phi x-i) \operatorname{sinc}(\varphi y-j) \operatorname{sinc}(\Phi u-i) \operatorname{sinc}(\varphi v-j) d x d y
\end{aligned}
$$




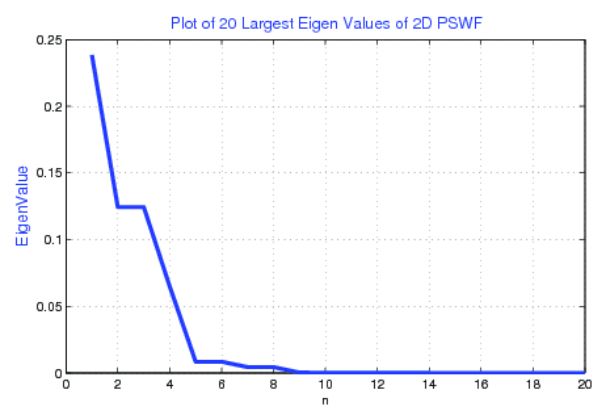

(a) Eigenvalues

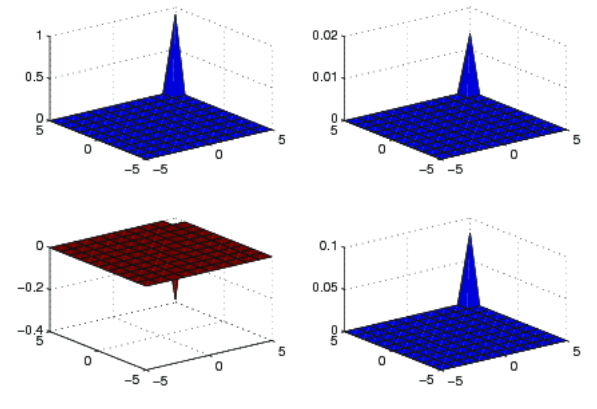

(b) First Four Eigenfunctions

Figure A.1: 2D PSWF. Computed using the sampling theorem approach.

Substituting (A.28) into (A.26), we obtain

$$
\begin{aligned}
\lambda \Psi(x, y)=\int_{-\gamma}^{\gamma} \int_{-\tau}^{\tau} \Psi(u, v) \sum_{i=-\infty}^{\infty} \sum_{j=\infty}^{\infty} \operatorname{sinc}(\Phi x-i) \operatorname{sinc}(\varphi y-j) \\
\quad \times \operatorname{sinc}(\Phi u-i) \operatorname{sinc}(\varphi v-j) d x d y \\
=\sum_{i=-\infty}^{\infty} \sum_{j=\infty}^{\infty} \operatorname{sinc}(\Phi x-i) \operatorname{sinc}(\varphi y-j) \int_{-\gamma}^{\gamma} \int_{-\tau}^{\tau} \Psi(u, v) \\
\times \operatorname{sinc}(\Phi u-i) \operatorname{sinc}(\varphi v-j) d x d y
\end{aligned}
$$

The integral in (A.29) can be evaluated using (A.26) as

$$
\lambda_{i j} \Psi(i, j)=\int_{-\gamma}^{\gamma} \int_{-\tau}^{\tau} \Psi(u, v) \operatorname{sinc}(\Phi u-i) \operatorname{sinc}(\varphi v-j) d x d y
$$

Expanding $\Psi(u, v)$ using the sampling theorem and substituting into (A.30), we have

$$
\begin{aligned}
\lambda_{i j} \Psi(i, j)= & \sum_{a=-\infty}^{\infty} \sum_{b=\infty}^{\infty} \int_{-\gamma}^{\gamma} \int_{-\tau}^{\tau} \operatorname{sinc}(\Phi u-i) \operatorname{sinc}(\varphi v-j) \operatorname{sinc}(\Phi u-a) \\
& \times \operatorname{sinc}(\varphi v-b) \Psi_{i j}(a, b) d u d v \\
= & \sum_{a=-\infty}^{\infty} \sum_{b=\infty}^{\infty}(\mathbf{A}(i, a) \mathbf{B}(j, b)) \Psi_{i j}(a, b)
\end{aligned}
$$


where $\mathbf{A}(i, a)$ and $\mathbf{B}(j, b)$ denotes the $1 \mathrm{D}$ integrals in (A.31) evaluated with respect to $u$ and $v$ respectively. A compact representation of (A.31) for all possible values of $i, j, a$ and $b$ is

$$
\lambda \Psi=\mathbf{P} \Psi
$$

where $\mathbf{P}=\mathbf{A} \otimes \mathbf{B}$. The $2 \mathrm{D}$ integral equation has now been reduced to a discrete eigenvalue problem with the infinite dimensional matrix $\mathbf{P}$. It has however been shown in literature [98] for the 1D case that the infinite matrices $\mathbf{A}$ and $\mathbf{B}$ have significant element in submatrices with dimension of the order of the space-bandwidth product. Matrix $\mathbf{P}$ can therefore be truncated to dimension $c=\gamma \tau \Phi \varphi$.

\section{Computation of 2D PSWF Eigenfunctions}

The procedures for the implementation of the discrete solution are:

- Calculate the Shannon number (i.e., space-bandwidth product) for $x$ and $y$-dimensions

- Perform 1D integrals numerically to obtain $\mathbf{A}$ and $\mathbf{B}$

- Truncate matrices A and B in the order of the Shannon's number

- Evaluate $\mathbf{P}$ and solve the eigenvalue problem in (A.32)

- Interpolate the eigenvectors to obtain the 2D PSWF eigenfunctions

Fig. A.1 present the eigenvalues and the first four eigenfunctions.

\section{A.4 Application to Wireless Channel Predic- tion}

This section addresses the problem of MIMO channel prediction using bandlimited extrapolation techniques. Although the iterative and non-iterative 
schemes presented in Section A.2 can be applied directly to extrapolate narrowband channels based on the knowledge that the Doppler spectra has a finite support, the section will focus on the application of Discrete prolate spheroidal wave sequences (DPSS) to the prediction of narrowband MIMO channels. Similar studies have been reported in [223,224] for time-variant SISO channel estimation. This approach will also be extended to the prediction of MIMO-OFDM channels, where the channel has finite support in both time and frequency domain (i.e., finite Doppler and delay bandwidths).

\section{A.4.1 Narrowband Channel}

Consider an $N \times M$ MIMO channel with each entry modeled as

$$
h(t)=\sum_{p=1}^{P} \alpha_{p} e^{j \omega_{p} t}
$$

where $\alpha_{p}$ is the complex attenuation which accounts for attenuation, phase shifts and antenna response and

$$
\omega_{p}=\omega_{\max } \cos \theta_{p}
$$

is the Doppler frequency, with $\omega_{\max }$ denoting the maximum Doppler frequency. Assume that the channel is sampled at an interval $T_{\mathrm{s}}$ and define $h[\ell] \triangleq h\left(\ell T_{\mathrm{s}}\right)$. (A.33) can then be represented as

$$
h(\ell)=\sum_{p=1}^{P} \alpha_{p} e^{j \ell \nu_{p}}
$$

where $\nu_{p}=\omega_{p} T_{\mathrm{s}}$ is the normalized Doppler frequency which can be expressed in terms of the normalized maximum Doppler frequency $\nu_{\max }$ as

$$
\nu_{p}=\nu_{\max } \cos \theta_{p}
$$

Since the angles of arrival typically vary between $-\pi$ and $\pi$, the Doppler spectral of the channel is bounded by $\left[-\nu_{\max }, \nu_{\max }\right]$. 


\section{Basis Expansion}

As shown by Slepian [178], an optimal basis for bandlimited channel are the DPSS, which are concentrated in an interval of length $L$ [224]

$$
\lambda=\frac{\sum_{\ell=0}^{L-1}|\Psi[\ell]|^{2}}{\sum_{\ell=-\infty}^{\infty}|\Psi[\ell]|^{2}}
$$

with $0 \leq \lambda \leq 1$ and bandlimited to $\left[-\nu_{\max },-\nu_{\max }\right]$ such that

$$
\Psi[\ell]=\int_{-\nu_{\max }}^{\nu_{\max }} \Psi^{f}(\nu) e^{j \ell \nu} d \nu
$$

where

$$
\Psi^{f}(\nu)=\sum_{\ell=-\infty}^{\infty} \Psi[\ell] e^{-j \ell \nu}
$$

The DPSS have been shown to be the real valued eigenvectors of [178]

$$
\sum_{m=0}^{L-1} \frac{\sin \left(2 \pi \nu_{\max }(m-\ell)\right)}{\pi(m-\ell)} \Psi_{k}[m]=\lambda_{k} \Psi_{k}[\ell]
$$

for $m, \ell \in[0,1, \cdots, L-1]$. Similar to the PSWF, the DPSS exhibits properties analogous to those highlighted in Section A.3. Note that the eigenvalues $\lambda_{k}$ of (A.39) are close to unity for $k \leq\left\lceil 2 \nu_{\max } L\right\rceil+1$ and rapidly decrease to zero for $k>\left\lceil 2 \nu_{\max } L\right\rceil+1$. The optimal dimension for a basis expansion using DPSS can therefore be approximated by $P=\left\lceil 2 \nu_{\max } L\right\rceil+1[223]$.

\section{Channel Prediction}

The first step in the prediction of the channel is to expand the $L$ noisy CSI samples in terms of the basis function

$$
\hat{h}[\ell]=\sum_{p=0}^{P^{\prime}-1} \gamma_{p} \Psi_{p}[\ell] \quad \ell=0,1, \cdots, L-1
$$


where $P^{\prime}$ is the model order which is satisfies $P \leq P^{\prime} \leq L$ and $\gamma_{p}$ are the weighting co-efficients. (A.41) can be expressed in matrix form as

$$
\left[\begin{array}{c}
\hat{h}[0] \\
\hat{h}[1] \\
\vdots \\
\hat{h}[L-1]
\end{array}\right]=\left[\begin{array}{cccc}
\Psi_{0}[0] & \Psi_{1}[0] & \cdots & \Psi_{P^{\prime}-1}[0] \\
\Psi_{0}[1] & \Psi_{1}[1] & \cdots & \Psi_{P^{\prime}-1}[1] \\
\vdots & \vdots & \ddots & \vdots \\
\Psi_{0}[L-1] & \Psi_{1}[L-1] & \cdots & \Psi_{P^{\prime}-1}[L-1]
\end{array}\right]\left[\begin{array}{c}
\gamma_{0} \\
\gamma_{1} \\
\vdots \\
\gamma_{P^{\prime}-1}
\end{array}\right]
$$

which can be expressed in a compact form

$$
\hat{\mathbf{h}}=\Psi \gamma
$$

Equation (A.43) is solved to obtain the weights vector $\gamma$ as

$$
\hat{\gamma}=\Psi^{\dagger} \hat{\mathbf{h}}
$$

where $\mathbf{A}^{\dagger}$ denotes the Pseudo-inverse of $\mathbf{A}$. Using the estimated basis, future states of the channel can be predicted from

$$
\tilde{h}[u]=\sum_{p=0}^{P^{\prime}-1} \hat{\gamma}_{p} \tilde{\Psi}_{p}[u] \quad u=L, L+1, \cdots
$$

where $\tilde{\Psi}_{p}[u]$ is the extrapolated basis vector at instant $u$ which can be obtained using [224]

$$
\tilde{\Psi}_{p}[u]=\frac{1}{\lambda_{p}} \sum_{\ell=0}^{L-1} \frac{\sin \left(2 \pi \nu_{\max }(\ell-u)\right)}{\pi(\ell-u)} \Psi_{p}[\ell]
$$

\section{A.4.2 Wideband Channel}

Here, the channel prediction method based on Slepian basis expansion is extended to wideband MIMO channels where the finite path delays result in an additional dimension in addition to the Doppler bandwidth. Again, the MIMO channel is treated on a per entry basis. Consider the impulse response of a frequency-selective channel defined as

$$
h(t, \tau)=\sum_{p=1}^{P} \alpha_{p} e^{j \omega_{p} t} \delta\left(\tau-\tau_{p}\right)
$$


where $\tau_{p}$ is used to denote the delay of the $p$ th path. The Fourier transformation of (A.47) gives the frequency response of the channel

$$
h(t, f)=\sum_{p=1}^{P} \alpha_{p} e^{j \omega_{p} t} e^{-j 2 \pi f \tau_{p}}
$$

where $f$ denotes the frequency variable. Now, assuming an OFDM based transmission with subcarrier spacing $T_{\mathrm{f}}$, the sampled frequency can be represented as

$$
h(\ell, b)=\sum_{p=1}^{P} \alpha_{p} e^{j n u_{p} \ell} e^{-j 2 \pi b \eta_{p}}
$$

where $b$ is the frequency index and $\eta_{p}=\tau_{p} T_{\mathrm{f}}$ is the normalized delay of the $p$ th path which satisfies

$$
0 \leq \eta_{p} \leq \eta_{\max }
$$

where $\eta_{\max }$ is the maximum normalized delay. Based on the knowledge of the symmetrical Doppler bandwidth and asymmetrical delay bandwidth, the doubly selective channel can be expanded using the 2D-DPSS.

\section{Basis Expansion}

Analogous to the 1D DPSS, the 2D-DPSS bandlimited to $\left[-\nu_{\max }, \nu_{\max }\right]$ and $\left[0, \eta_{\max }\right.$ and concentrated on intervals $[0, L-1]$ and $[0, Q]$ are the eigenvectors of

$$
\sum_{m=0}^{L-1} \sum_{b=0}^{Q-1} \frac{\sin \left(2 \pi \nu_{\max }(m-\ell)\right)}{\pi(m-\ell)} \frac{\sin \left(2 \pi \eta_{\max }(b-v)\right)}{\pi(b-v)} \Psi_{k}[m, b]=\lambda_{k} \Psi_{k}[\ell, v]
$$

for $k \in[0,1, \cdots,(L-1)(Q-1)]$. Denoting $\Psi_{k}=\left[\Psi_{k}[0,0], \Psi_{k}[1,0], \cdots, \Psi_{k}[L-\right.$ $\left.1,0], \Psi_{k}[0,1], \Psi_{k}[1,1], \cdots, \Psi_{k}[L-1,1], \cdots, \Psi_{k}[L-1, Q-1]\right]$, (A.51) can be compactly written as

$$
\mathbf{E} \boldsymbol{\Psi}_{k}=\lambda_{k} \boldsymbol{\Psi}_{k}
$$

where

$$
\mathbf{E}=\mathbf{C} \otimes \mathbf{D}
$$


where $\otimes$ denotes the Kronecker product. Matrices $\mathbf{C}$ and $\mathbf{D}$ are defined as

$$
[\mathbf{C}]_{k, \ell}=\frac{\sin \left(2 \pi \nu_{\max }(k-\ell)\right)}{\pi(k-\ell)} \quad k, \ell \in[0,1, \cdots, L-1]
$$

and

$$
[\mathbf{D}]_{k, b}=\frac{\sin \left(2 \pi \eta_{\max }(k-b)\right)}{\pi(k-b)} \quad k, b \in[0,1, \cdots, Q-1]
$$

respectively. Note that the eigenvalue problem in (A.52) can be written as

$$
(\mathbf{C} \otimes \mathbf{D})\left(\boldsymbol{\Psi}_{k}^{\mathrm{t}} \otimes \boldsymbol{\Psi}_{k}^{\mathrm{f}}\right)=\left(\boldsymbol{\lambda}_{k}^{\mathrm{t}} \otimes \boldsymbol{\lambda}_{k}^{\mathrm{f}}\right)\left(\boldsymbol{\Psi}_{k}^{\mathrm{t}} \otimes \boldsymbol{\Psi}_{k}^{\mathrm{f}}\right)
$$

where $\boldsymbol{\Psi}_{k}^{\mathrm{t}}$ and $\boldsymbol{\lambda}_{k}^{\mathrm{t}}$ are the eigenvectors and eigenvalues of $\mathbf{C}$, respectively. The eigenvectors and eigenvalues of $\mathbf{D}$ are contained in $\boldsymbol{\Psi}_{k}^{\mathrm{f}}$ and $\boldsymbol{\lambda}_{k}^{\mathrm{f}}$, respectively.

\section{Channel Prediction}

Similar to the narrowband case, the known channel is expanded in terms of the Slepian basis, thus

$$
\hat{g}[\ell, q]=\sum_{p=0}^{P^{\prime}-1} \gamma_{p} \Psi_{p}[\ell, q]
$$

for $\ell=0,1, \cdots, L-1$ and $q=0,1, \cdots, Q-1$. Here, the model order $P^{\prime}$ satisfies the constraint

$$
\left\lceil 2 \nu_{\max } \eta_{\max } L Q\right\rceil+1 \leq P^{\prime} \leq(L-1)(Q-1)
$$

To allow for the estimation of the weights, let us now put (A.57) into the matrix formula

$$
\hat{\mathrm{g}}=\Psi \gamma
$$

where

$$
\begin{gathered}
\hat{\mathbf{g}}=[\hat{g}[0,0], \hat{g}[1,0], \cdots, \hat{g}[L-1,0], \hat{g}[0,1], \cdots, \hat{g}[L-1,1], \cdots, \hat{g}[L-1, Q-1]], \\
\gamma=\left[\gamma_{0}, \gamma_{1}, \gamma_{2}, \cdots, \gamma_{P^{\prime}-1}\right]
\end{gathered}
$$


and

$$
\Psi=\left[\begin{array}{ccc}
\Psi_{0}^{\mathrm{t}}[0] & \cdots & \Psi_{P^{\prime}-1}^{\mathrm{t}}[0] \\
\Psi_{0}^{\mathrm{t}}[1] & \cdots & \Psi_{P^{\prime}-1}^{\mathrm{t}}[1] \\
\vdots & \ddots & \vdots \\
\Psi_{0}^{\mathrm{t}}[L-1] & \cdots & \Psi_{P^{\prime}-1}^{\mathrm{t}}[L-1]
\end{array}\right] \otimes\left[\begin{array}{ccc}
\Psi_{0}^{\mathrm{f}}[0] & \cdots & \Psi_{P^{\prime}-1}^{\mathrm{f}}[0] \\
\Psi_{0}^{\mathrm{f}}[1] & \cdots & \Psi_{P^{\prime}-1}^{\mathrm{f}}[1] \\
\vdots & \ddots & \vdots \\
\Psi_{0}^{\mathrm{f}}[Q-1] & \cdots & \Psi_{P^{\prime}-1}^{\mathrm{f}}[Q-1]
\end{array}\right]
$$

The weights $\gamma$ can now be estimated from (A.44), thus

$$
\hat{\gamma}=\Psi^{\dagger} \hat{\mathrm{g}}
$$

\section{A.5 Simulation and Results}

In this section we evaluate the prediction performance of the DPSS based method and compare this with the application of the 1D ESPRIT based approach of [24]. We consider a $2 \times 2$ MIMO channel with $P=10$ paths and a mobile velocity, $v=50 \mathrm{kmp} / \mathrm{h}$. The AOA and AOD are drawn from a uniform distribution as $\theta_{p}, \phi_{p} \sim \mathcal{U}[0,2 \pi)$ and the complex amplitudes are generated as $\alpha_{p} \sim \mathcal{C N}(0,1)$.

Fig. A.4, we present the NMSE versus prediction horizon at $\mathrm{SNR}=\{10,20\} \mathrm{dB}$ and a sampling interval of $200 \mathrm{~ms}$. We observe that while both methods show similar performance over the training segment, the DPSS performs poorly in the prediction segment. A similar observation is made in Fig. A.5, where we plot the CDF of NSE at a prediction interval of $1 \lambda$. In Fig. A.6, we show the NMSE as a function of prediction horizon with a sampling interval of $T_{\text {samp }}=20 \mu s$. It shows that the ESPRIT based scheme still performs better. However, the performance difference in the prediction segment is negligible when compared to Fig. A.4. This shows that increasing the sampling rate improves the performance of the DPSS based method. The corresponding CDF is shown in Fig. A.7. It shows that the performance of both schemes are closer at lower SNR than higher SNR. We saw an NMSE difference of about $7 \mathrm{~dB}$ at $\mathrm{SNR}=30 \mathrm{~dB}, 4 \mathrm{~dB}$ at $\mathrm{SNR}=20 \mathrm{~dB}$ and $<0.5 \mathrm{~dB}$ at SNR $=20 \mathrm{~dB}$. 


\section{A.6 Summary}

This chapter presented a summary of our investigations on the prediction of MIMO channels using bandlimited extrapolation techniques. We presented a method for solving 2D PSWF using the sampling theorem. Methods for predicting narrowband and wideband channels are also developed using the DPSS and comparison was made with parametric prediction using 1D ESPRIT based schemes. 

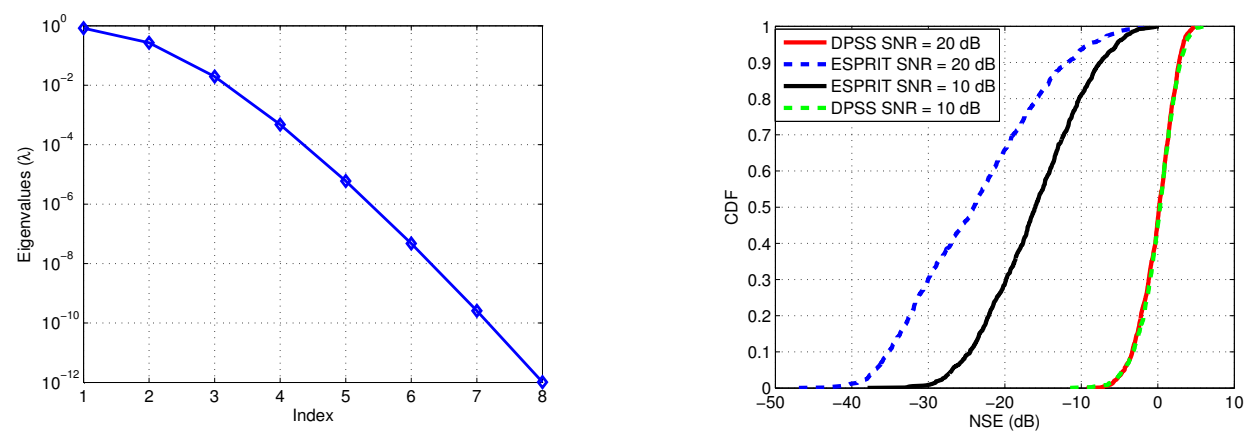

Figure A.2: Eigenvalues of the DPSS for a channel with $f_{c}=2.0 \mathrm{GHz}, v=$ Figure A.5: CDF of NSE for a $2 \times 2$ $75 \mathrm{kmph}, T_{\mathrm{samb}}=20 \mu \mathrm{s}$, and $K=200$.

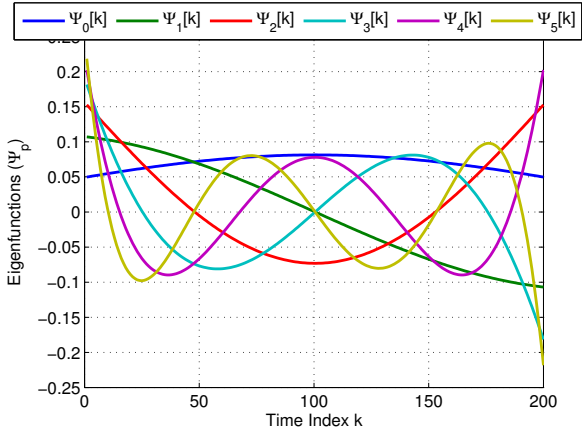

MIMO channel with $P=10$ and $T_{\text {samn }}=20 \mu s$.

Figure A.3: First five DPSS for a Figure A.6: NMSE versus prediction channel with with $f_{c}=2.0 \mathrm{GHz}, v=$ horizon for a $2 \times 2$ MIMO channel $75 \mathrm{kmph}, T_{\mathrm{samb}}=20 \mu \mathrm{s}$, and $K=200$. with $P=10$ and $T_{\mathrm{samb}}=200 \mathrm{~ms}$.
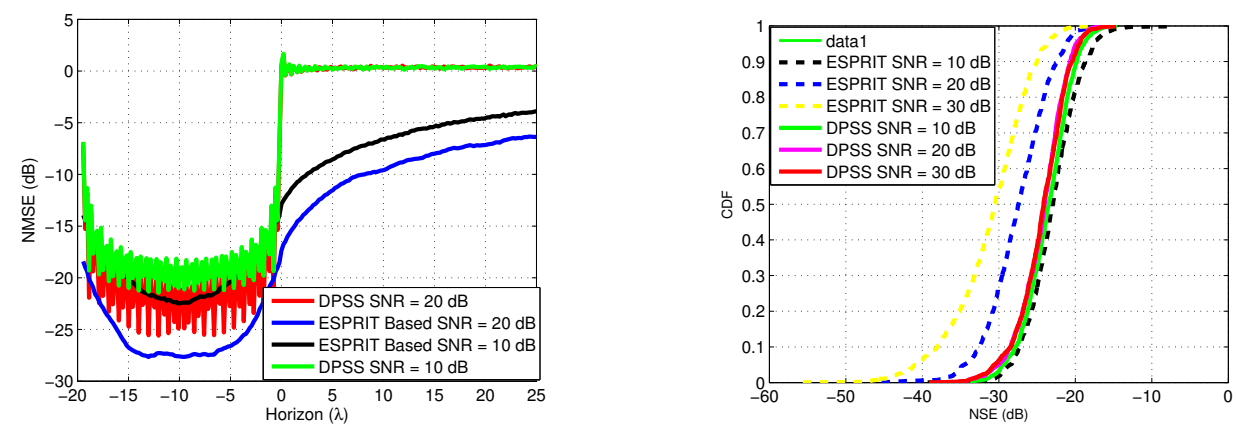

Figure A.4: NMSE versus prediction Figure A.7: CDF of NSE for a $2 \times 2$ horizon for a $2 \times 2$ MIMO channel MIMO channel with $P=10$ and with $P=10$ and $T_{\text {samp }}=20 \mu \mathrm{s} . \quad T_{\text {samp }}=200 \mathrm{~ms}$. 


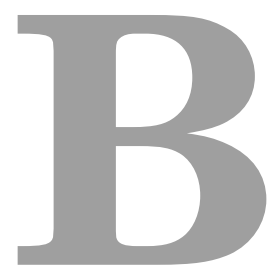

\section{Proof of Performance Bound} Expressions

\section{B.1 Derivatives for the FIM in Narrowband Error Bound}

\footnotetext{
7 HIS section presents the derivatives of the observation vector $\mathbf{h}$ required 1 for evaluating the error bound in Section 8.1.1.
} 


\section{B.1.1 Derivative with Respect to $\mathfrak{R}(\boldsymbol{\alpha})$}

$$
\begin{aligned}
\frac{\partial \mathbf{h}}{\partial \mathfrak{R}\left(\alpha_{p}\right)} & =\frac{\partial\left(\mathbf{A}_{r} \diamond \mathbf{A}_{t} \diamond \mathbf{A}_{d}\right) \boldsymbol{\alpha}}{\partial \mathfrak{R}\left(\alpha_{p}\right)} \\
& =\left(\mathbf{A}_{r} \diamond \mathbf{A}_{t} \diamond \mathbf{A}_{d}\right) \frac{\partial \boldsymbol{\alpha}}{\partial \alpha_{p}} \\
& =\left(\mathbf{A}_{r} \diamond \mathbf{A}_{t} \diamond \mathbf{A}_{d}\right) \boldsymbol{\Pi}_{P}
\end{aligned}
$$

where $\Pi_{k}$ is a $P \times 1$ vector having a 1 as the $p$ th element and all other elements zero. (B.1) can further be simplified to

$$
\begin{aligned}
\frac{\partial \mathbf{h}}{\partial \mathfrak{R}\left(\alpha_{p}\right)} & =\left[\mathbf{A}_{r}\right]_{:, p} \otimes\left[\mathbf{A}_{t}\right]_{:, p} \otimes\left[\mathbf{A}_{d}\right]_{:, p} \\
& =\left[\mathbf{A}_{r} \diamond \mathbf{A}_{t} \diamond \mathbf{A}_{d}\right]_{:, p} \\
& =[\mathbf{A}]_{:, p}
\end{aligned}
$$

where $[\mathbf{B}]_{:, p}$ denotes the $p$ th column of B. Using (B.2), the derivative with respect to $\mathfrak{R}(\boldsymbol{\alpha})$ is

$$
\begin{aligned}
\frac{\partial \mathbf{h}}{\partial \mathfrak{R}(\boldsymbol{\alpha})} & =\left[\begin{array}{llll}
\frac{\partial \mathbf{h}}{\partial \mathfrak{R}\left(\alpha_{1}\right)} & \frac{\partial \mathbf{h}}{\partial \mathfrak{R}\left(\alpha_{2}\right)} & \cdots & \frac{\partial \mathbf{h}}{\partial \mathfrak{R}\left(\beta_{P}\right)}
\end{array}\right] \\
& =\left[\begin{array}{llll}
{[\mathbf{A}]_{:, 1}} & \cdots & {[\mathbf{A}]_{:, P}}
\end{array}\right] \\
& =\mathbf{A}
\end{aligned}
$$

\section{B.1.2 Derivative with Respect to $\mathfrak{I}(\boldsymbol{\alpha})$}

$$
\begin{aligned}
\frac{\partial \mathbf{h}}{\partial \mathfrak{I}\left(\alpha_{p}\right)} & =\left(\mathbf{A}_{r} \diamond \mathbf{A}_{t} \diamond \mathbf{A}_{d}\right) \frac{\partial \boldsymbol{\alpha}}{\partial \mathfrak{I}\left(\alpha_{p}\right)} \\
& =j\left(\mathbf{A}_{r} \diamond \mathbf{A}_{t} \diamond \mathbf{A}_{d}\right) \mathbf{\Pi}_{P} \\
& =j \mathbf{A}
\end{aligned}
$$




\section{B.1.3 Derivative with Respect to $\mu^{\mathrm{r}}$}

$$
\begin{aligned}
\frac{\partial \mathbf{h}}{\partial \mu_{p}^{\mathrm{r}}} & =\left(\frac{\partial \mathbf{A}_{r}}{\partial \mu_{k}^{\mathrm{r}}} \diamond \mathbf{A}_{t} \diamond \mathbf{A}_{d}\right) \boldsymbol{\alpha} \\
& =\left(\frac{\partial\left[\mathbf{A}_{r}\right]_{:, p}}{\partial \mu_{p}^{\mathrm{r}}} \otimes\left[\mathbf{A}_{t}\right]_{:, p} \otimes\left[\mathbf{A}_{d}\right]_{:, p}\right) \alpha_{p}
\end{aligned}
$$

Using (B.6), we obtain

$$
\begin{aligned}
& \frac{\partial \mathbf{h}}{\partial \boldsymbol{\mu}^{\mathrm{r}}}=\left[\begin{array}{llll}
\frac{\partial \mathbf{h}}{\partial \mu_{1}^{\mathrm{r}}} & \frac{\partial \mathbf{h}}{\partial \mu_{2}^{\mathrm{r}}} & \cdots & \frac{\partial \mathbf{h}}{\partial \mu_{P}^{\mathrm{r}}}
\end{array}\right] \\
& =\left[\left(\frac{\partial\left[\mathbf{A}_{r}\right]_{:, 1}}{\partial \mu_{1}^{\mathrm{r}}} \otimes\left[\mathbf{A}_{t}\right]_{:, 1} \otimes\left[\mathbf{A}_{d}\right]_{:, 1}\right) \beta_{1} \quad \cdots\right. \\
& \left.\left(\frac{\partial\left[\mathbf{A}_{r}\right]_{:, P}}{\partial \mu_{P}^{\mathrm{r}}} \otimes\left[\mathbf{A}_{t}\right]_{:, P} \otimes\left[\mathbf{A}_{d}\right]_{:, P}\right) \alpha_{P}\right] \\
& =\left(\mathbf{D}_{r} \diamond \mathbf{A}_{t} \diamond \mathbf{A}_{d}\right) \mathbf{X}
\end{aligned}
$$

where

$$
\mathbf{D}_{r}=\left[\begin{array}{llll}
\frac{\partial\left[\mathbf{A}_{r}\right]_{:, 1}}{\partial \mu_{1}^{\mathrm{r}}} & \frac{\partial\left[\mathbf{A}_{r}\right]_{:, 2}}{\partial \mu_{2}^{\mathrm{r}}} & \cdots & \frac{\partial\left[\mathbf{A}_{r}\right]_{:, P}}{\partial \mu_{P}^{\mathrm{r}}}
\end{array}\right]
$$

For the ULA, $\mathbf{D}_{r}$ can be found using (4.26) to be

$$
\mathbf{D}_{\mathrm{r}}=\left[\begin{array}{ccc}
0 & \cdots & 0 \\
j e^{j \nu_{1}^{\mathrm{r}}} & \cdots & j e^{j \nu_{P}^{\mathrm{r}}} \\
2 j e^{j 2 \nu_{1}^{\mathrm{r}}} & \cdots & 2 j e^{j 2 \nu_{P}^{\mathrm{r}}} \\
\vdots & \ddots & \vdots \\
j(N-1) e^{j(M-1) \nu_{1}^{\mathrm{r}}} & \cdots & j(M-1) e^{j(N-1) \nu_{P}^{\mathrm{r}}}
\end{array}\right]
$$

Equation (B.8) can be expressed in terms of $\mathbf{A}_{\mathbf{r}}$ as

$$
\mathbf{D}_{\mathrm{r}}=j\left[\begin{array}{cccc}
0 & 0 & \cdots & 0 \\
0 & 1 & \cdots & 0 \\
\vdots & \vdots & \ddots & \vdots \\
0 & 0 & \cdots & (M-1)
\end{array}\right] \mathbf{A}_{\mathrm{r}}
$$

$\mathbf{X}$ is a diagonal matrix with the complex amplitudes $\boldsymbol{\alpha}$ on its diagonal. 


\section{B.1.4 Derivative with Respect to $\mu^{\mathrm{t}}$}

$$
\begin{aligned}
& \frac{\partial \mathbf{h}}{\partial \boldsymbol{\mu}^{\mathrm{t}}}=\left[\begin{array}{llll}
\frac{\partial \mathbf{h}}{\partial \mu_{1}^{\mathrm{t}}} & \frac{\partial \mathbf{h}}{\partial \mu_{2}^{\mathrm{t}}} & \cdots & \frac{\partial \mathbf{h}}{\partial \mu_{P}^{\mathrm{t}}}
\end{array}\right] \\
& =\left[\left(\left[\mathbf{A}_{r}\right]_{:, 1} \otimes \frac{\partial\left[\mathbf{A}_{t}\right]_{:, 1}}{\partial \mu_{1}^{t}} \otimes\left[\mathbf{A}_{d}\right]_{:, 1}\right) \alpha_{1} \quad \ldots\right. \\
& \left.\left(\left[\mathbf{A}_{r}\right]_{:, P} \otimes \frac{\partial\left[\mathbf{A}_{t}\right]_{:, P}}{\partial \mu_{P}^{\mathrm{t}}} \otimes\left[\mathbf{A}_{d}\right]_{:, P}\right) \alpha_{P}\right] \\
& =\left(\mathbf{A}_{r} \diamond \mathbf{D}_{t} \diamond \mathbf{A}_{d}\right) \mathbf{X}
\end{aligned}
$$

where $\mathbf{D}_{\mathrm{t}}$ is defined similar to $\mathbf{D}_{\mathrm{r}}$.

\section{B.1.5 Derivative with Respect to $\nu$}

$$
\begin{aligned}
& \frac{\partial \mathbf{h}}{\partial \boldsymbol{\nu}}=\left[\begin{array}{llll}
\frac{\partial \mathbf{h}}{\partial \nu_{1}} & \frac{\partial \mathbf{h}}{\partial \nu_{2}} & \cdots & \frac{\partial \mathbf{h}}{\partial \nu_{P}}
\end{array}\right] \\
& =\left[\left(\left[\mathbf{A}_{r}\right]_{:, 1} \otimes\left[\mathbf{A}_{t}\right]_{:, 1} \otimes \frac{\partial\left[\mathbf{A}_{d}\right]_{:, 1}}{\partial \nu_{1}}\right) \alpha_{1} \quad \ldots\right. \\
& \left.\left(\left[\mathbf{A}_{r}\right]_{:, P} \otimes\left[\mathbf{A}_{t}\right]_{:, P}\right) \alpha_{P} \otimes \frac{\partial\left[\mathbf{A}_{d}\right]_{:, P}}{\partial \nu_{P}}\right] \\
& =\left(\mathbf{A}_{r} \diamond \mathbf{A}_{t} \diamond \mathbf{D}_{d}\right) \mathbf{X}
\end{aligned}
$$

where $\mathbf{D}_{d}$ is defined similar to (B.8).

\section{B.2 Evaluation of FIM and Error Bound}

The simplified expression for the FIM and the prediction error bound are

obtained using the derivatives in Appendix B.1. Using (8.25) and (8.26), the matrix $\mathbf{J}$ in (8.27) is given by

$$
\mathfrak{J}=\frac{\partial \mathbf{h}}{\partial \boldsymbol{\Theta}} \frac{\partial \mathbf{h}}{\partial \boldsymbol{\Theta}}
$$


where

$$
\begin{aligned}
& \frac{\partial \mathbf{h}}{\partial \boldsymbol{\Theta}}=\left[\begin{array}{lllll}
\frac{\partial \mathbf{h}}{\partial \mathfrak{R}(\boldsymbol{\alpha})} & \frac{\partial \mathbf{h}}{\partial \mathfrak{I}(\boldsymbol{\alpha})} & \frac{\partial \mathbf{h}}{\partial \boldsymbol{\mu}^{\mathrm{r}}} & \frac{\partial \mathbf{h}}{\partial \boldsymbol{\mu}^{\mathrm{t}}} & \frac{\partial \mathbf{h}}{\partial \boldsymbol{\nu}}
\end{array}\right]
\end{aligned}
$$

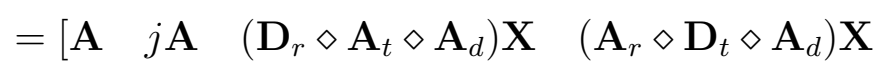

$$
\begin{aligned}
& \left.\left(\mathbf{A}_{r} \diamond \mathbf{A}_{t} \diamond \mathbf{D}_{d}\right) \mathbf{X}\right]
\end{aligned}
$$

With $\mathbf{P}_{1}-\mathbf{P}_{4}$ given in (8.19)-(50), (B.13) can be simplified to

$$
\frac{\partial \mathbf{h}}{\partial \boldsymbol{\Phi}}=\mathbf{P}_{1} \diamond \mathbf{P}_{2} \diamond \mathbf{P}_{3} \diamond \mathbf{P}_{4}
$$

and thus (B.12) becomes

$$
\mathfrak{J}=\left(\mathbf{P}_{1} \diamond \mathbf{P}_{2} \diamond \mathbf{P}_{3} \diamond \mathbf{P}_{4}\right)^{H}\left(\mathbf{P}_{1} \diamond \mathbf{P}_{2} \diamond \mathbf{P}_{3} \diamond \mathbf{P}_{4}\right)
$$

Using the properties of the Khatri-Rao and Hadamard products, (B.15) reduces to the form given in (8.26).

\section{B.3 Derivatives for the FIM for Wideband Er- ror Bound}

The derivatives of the covariance matrix and observation vector $\mathbf{h}$ required for evaluating the FIM expression as given in (8.40) are given in this section.

\section{B.3.1 Derivative with Respect $\sigma^{2}$}

Based on the independent Gaussian noise assumption, the derivative of the covariance matrix $\mathbf{C}=\sigma^{2} \mathbf{I}$ is thus

$$
\frac{\partial \mathbf{C}}{\partial \sigma^{2}}=\mathbf{I}
$$

On substituting (B.16) into (8.12), the entry of the FIM dependent on noise variance is obtained as

$$
\operatorname{Tr}\left[\mathbf{C}^{-1} \frac{\partial \mathbf{C}}{\partial \boldsymbol{\Theta}} \mathbf{C}^{-1} \frac{\partial \mathbf{C}}{\partial \boldsymbol{\Theta}}\right]=\frac{P K N M}{\sigma^{4}}
$$




\section{B.3.2 Derivative with Respect to $\mathfrak{R}(\boldsymbol{\alpha})$}

$$
\begin{aligned}
\frac{\partial \mathbf{h}}{\partial \mathfrak{R}\left(\alpha_{p}\right)} & =\frac{\partial\left(\mathbf{A}_{r} \diamond \mathbf{A}_{t} \diamond \mathbf{A}_{d} \diamond \mathbf{A}_{f}\right) \boldsymbol{\alpha}}{\partial \mathfrak{R}\left(\alpha_{p}\right)} \\
& =\left(\mathbf{A}_{r} \diamond \mathbf{A}_{t} \diamond \mathbf{A}_{d} \diamond \mathbf{A}_{f}\right) \frac{\partial \boldsymbol{\alpha}}{\partial \alpha_{p}} \\
& =\left(\mathbf{A}_{r} \diamond \mathbf{A}_{t} \diamond \mathbf{A}_{d} \diamond \mathbf{A}_{f}\right) \boldsymbol{\Pi}_{p}
\end{aligned}
$$

Equation (B.1) can further be simplified to

$$
\begin{aligned}
\frac{\partial \mathbf{h}}{\partial \mathfrak{R}\left(\alpha_{p}\right)} & =\left[\mathbf{A}_{r}\right]_{:, p} \otimes\left[\mathbf{A}_{t}\right]_{:, p} \otimes\left[\mathbf{A}_{d}\right]_{:, p} \otimes\left[\mathbf{A}_{f}\right]_{:, p} \\
& =\left[\mathbf{A}_{r} \diamond \mathbf{A}_{t} \diamond \mathbf{A}_{d} \diamond \mathbf{A}_{f}\right]_{:, p} \\
& =[\mathbf{A}]_{:, p}
\end{aligned}
$$

Using (B.19), the derivative with respect to $\Re(\boldsymbol{\alpha})$ is

$$
\begin{aligned}
\frac{\partial \mathbf{h}}{\partial \mathfrak{R}(\boldsymbol{\alpha})} & =\left[\begin{array}{llll}
\frac{\partial \mathbf{h}}{\partial \mathfrak{R}\left(\alpha_{1}\right)} & \frac{\partial \mathbf{h}}{\partial \mathfrak{R}\left(\alpha_{2}\right)} & \cdots & \frac{\partial \mathbf{h}}{\partial \mathfrak{R}\left(\alpha_{P}\right)}
\end{array}\right] \\
& =\left[\begin{array}{lll}
{[\mathbf{A}]_{;, 1}} & \cdots & {[\mathbf{A}]_{:, P}}
\end{array}\right] \\
& =\mathbf{A}
\end{aligned}
$$

\section{B.3.3 Derivative with Respect to $\mathfrak{I}(\boldsymbol{\alpha})$}

$$
\begin{aligned}
\frac{\partial \mathbf{h}}{\partial \Im\left(\alpha_{p}\right)} & =\left(\mathbf{A}_{r} \diamond \mathbf{A}_{t} \diamond \mathbf{A}_{d} \diamond \mathbf{A}_{f}\right) \frac{\partial \boldsymbol{\alpha}}{\partial \widetilde{I}\left(\alpha_{p}\right)} \\
& =j\left(\mathbf{A}_{r} \diamond \mathbf{A}_{t} \diamond \mathbf{A}_{d} \diamond \mathbf{A}_{f}\right) \boldsymbol{\Pi}_{P} \\
& =j \mathbf{A}
\end{aligned}
$$




\section{B.3.4 Derivative with Respect to $\mu^{\mathrm{r}}$}

$$
\begin{aligned}
\frac{\partial \mathbf{h}}{\partial \mu_{p}^{\mathrm{r}}} & =\left(\frac{\partial \mathbf{A}_{r}}{\partial \mu_{z}^{\mathrm{r}}} \mathbf{A}_{t} \diamond \mathbf{A}_{d} \diamond \mathbf{A}_{f}\right) \boldsymbol{\alpha} \\
& =\left(\frac{\partial\left[\mathbf{A}_{r}\right]_{:, p}}{\partial \mu_{p}^{\mathrm{r}}} \otimes\left[\mathbf{A}_{t}\right]_{:, p} \otimes\left[\mathbf{A}_{d}\right]_{:, p} \otimes\left[\mathbf{A}_{\tau}\right]_{:, p}\right) \alpha_{p}
\end{aligned}
$$

Using (B.22), we obtain

$$
\begin{aligned}
& \frac{\partial \mathbf{h}}{\partial \boldsymbol{\mu}^{\mathrm{r}}}=\left[\begin{array}{llll}
\frac{\partial \mathbf{h}}{\partial \mu_{1}^{\mathrm{r}}} & \frac{\partial \mathbf{h}}{\partial \mu_{2}^{\mathrm{r}}} & \cdots & \frac{\partial \mathbf{h}}{\partial \mu_{P}^{\mathrm{r}}}
\end{array}\right] \\
& =\left[\left(\frac{\partial\left[\mathbf{A}_{r}\right]_{:, 1}}{\partial \mu_{1}^{\mathrm{r}}} \otimes\left[\mathbf{A}_{t}\right]_{:, 1} \otimes\left[\mathbf{A}_{d}\right]_{:, 1} \otimes\left[\mathbf{A}_{\tau}\right]_{:, 1}\right) \alpha_{1} \quad \cdots\right. \\
& \left.\left(\frac{\partial\left[\mathbf{A}_{r}\right]_{:, P}}{\partial \mu_{P}^{\mathrm{r}}} \otimes\left[\mathbf{A}_{t}\right]_{:, P} \otimes\left[\mathbf{A}_{d}\right]_{:, P} \otimes\left[\mathbf{A}_{f}\right]_{:, P}\right) \alpha_{P}\right] \\
& =\left(\mathbf{D}_{r} \diamond \mathbf{A}_{t} \diamond \mathbf{A}_{d} \diamond \mathbf{A}_{f}\right) \mathbf{X}
\end{aligned}
$$

where

$$
\mathbf{D}_{r}=\left[\begin{array}{llll}
\frac{\partial\left[\mathbf{A}_{r}\right]_{:, 1}}{\partial \mu_{1}^{\mathrm{r}}} & \frac{\partial\left[\mathbf{A}_{r}\right]_{:, 2}}{\partial \mu_{2}^{\mathrm{r}}} & \cdots & \frac{\partial\left[\mathbf{A}_{r}\right]_{:, P}}{\partial \mu_{P}^{\mathrm{r}}}
\end{array}\right]
$$

which can be expressed in terms of $\mathbf{A}_{\mathrm{r}}$ as

$$
\mathbf{D}_{\mathrm{r}}=j\left[\begin{array}{cccc}
0 & 0 & \cdots & 0 \\
0 & 1 & \cdots & 0 \\
\vdots & \vdots & \ddots & \vdots \\
0 & 0 & \cdots & (M-1)
\end{array}\right] \mathbf{A}_{\mathrm{r}}
$$

\section{B.3.5 Derivative with Respect to $\boldsymbol{\mu}^{\mathrm{t}}$}

$$
\begin{aligned}
& \frac{\partial \mathbf{h}}{\partial \boldsymbol{\mu}^{\mathrm{t}}}=\left[\begin{array}{llll}
\frac{\partial \mathbf{h}}{\partial \mu_{1}^{\mathrm{t}}} & \frac{\partial \mathbf{h}}{\partial \mu_{2}^{\mathrm{t}}} & \cdots & \frac{\partial \mathbf{h}}{\partial \mu_{P}^{\mathrm{t}}}
\end{array}\right] \\
& =\left[\left(\left[\mathbf{A}_{r}\right]_{:, 1} \otimes \frac{\partial\left[\mathbf{A}_{t}\right]_{:, 1}}{\partial \mu_{1}^{t}} \otimes\left[\mathbf{A}_{d}\right]_{:, 1} \otimes\left[\mathbf{A}_{f}\right]_{:, 1}\right) \alpha_{1} \quad \cdots\right. \\
& \left.\left(\left[\mathbf{A}_{r}\right]_{;, P} \otimes \frac{\partial\left[\mathbf{A}_{t}\right]_{:, P}}{\partial \mu_{P}^{\mathrm{t}}} \otimes\left[\mathbf{A}_{d}\right]_{:, P} \otimes\left[\mathbf{A}_{f}\right]_{;, P}\right) \alpha_{P}\right] \\
& =\left(\mathbf{A}_{r} \diamond \mathbf{D}_{t} \diamond \mathbf{A}_{d} \diamond \mathbf{A}_{f}\right) \mathbf{X}
\end{aligned}
$$




\section{B.3.6 Derivative with Respect to $\nu$}

$$
\begin{aligned}
& \frac{\partial \mathbf{h}}{\partial \boldsymbol{\nu}}=\left[\begin{array}{llll}
\frac{\partial \mathbf{h}}{\partial \nu_{1}} & \frac{\partial \mathbf{h}}{\partial \nu_{2}} & \cdots & \frac{\partial \mathbf{h}}{\partial \nu_{P}}
\end{array}\right] \\
& =\left[\left(\left[\mathbf{A}_{r}\right]_{:, 1} \otimes\left[\mathbf{A}_{t}\right]_{:, 1} \otimes \frac{\partial\left[\mathbf{A}_{d}\right]_{:, 1}}{\partial \nu_{1}} \otimes\left[\mathbf{A}_{f}\right]_{:, 1}\right) \alpha_{1} \quad \ldots\right. \\
& \left.\left(\left[\mathbf{A}_{r}\right]_{:, P} \otimes\left[\mathbf{A}_{t}\right]_{:, P}\right) \alpha_{P} \otimes \frac{\partial\left[\mathbf{A}_{d}\right]_{:, P}}{\partial \nu_{P}} \otimes\left[\mathbf{A}_{f}\right]_{:, P}\right] \\
& =\left(\mathbf{A}_{r} \diamond \mathbf{A}_{t} \diamond \mathbf{D}_{d} \diamond \mathbf{A}_{f}\right) \mathbf{X}
\end{aligned}
$$

\section{B.3.7 Derivative with Respect to $\eta$}

Following a procedure similar to those presented above, the derivative of $\mathbf{h}$ with respect to $\boldsymbol{\eta}$ can be shown to be

$$
\frac{\partial \mathbf{h}}{\partial \boldsymbol{\eta}}=\left(\mathbf{A}_{r} \diamond \mathbf{A}_{t} \diamond \mathbf{A}_{d} \diamond \mathbf{D}_{f}\right) \mathbf{X}
$$

\section{B.4 Evaluation of FIM and Error Bound}

Once the derivatives of the channel observation $\mathbf{h}$ and the covariance matrix have been obtained, the expression for the FIM and the prediction error bound are obtained by substituting the derivatives in Appendix B.3. Using (8.25) and (8.100), the matrix $\mathfrak{J}$ in (8.51) is obtained as

$$
\mathfrak{J}=\frac{\partial \mathbf{h}}{\partial \Theta}^{H} \frac{\partial \mathbf{h}}{\partial \boldsymbol{\Theta}}
$$

where

$$
\begin{aligned}
& \frac{\partial \mathbf{h}}{\partial \boldsymbol{\Theta}}=\left[\begin{array}{llllll}
\frac{\partial \mathbf{h}}{\partial \mathfrak{R}(\boldsymbol{\alpha})} & \frac{\partial \mathbf{h}}{\partial \mathfrak{I}(\boldsymbol{\alpha})} & \frac{\partial \mathbf{h}}{\partial \boldsymbol{\mu}^{\mathrm{r}}} & \frac{\partial \mathbf{h}}{\partial \boldsymbol{\mu}^{\mathrm{t}}} & \frac{\partial \mathbf{h}}{\partial \boldsymbol{\nu}} & \frac{\partial \mathbf{h}}{\partial \boldsymbol{\eta}}
\end{array}\right]
\end{aligned}
$$

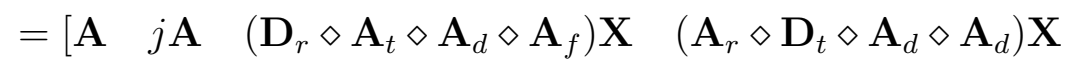

$$
\begin{aligned}
& \left.\left(\mathbf{A}_{r} \diamond \mathbf{A}_{t} \diamond \mathbf{D}_{d} \diamond \mathbf{A}_{d}\right) \mathbf{X} \quad\left(\mathbf{A}_{r} \diamond \mathbf{A}_{t} \diamond \mathbf{A}_{d} \diamond \mathbf{D}_{f}\right) \mathbf{X}\right]
\end{aligned}
$$


With $\mathbf{P}_{1}-\mathbf{P}_{5}$ as given in (8.41)-(8.45), (B.30) can be simplified to

$$
\frac{\partial \mathbf{h}}{\partial \boldsymbol{\Theta}}=\mathbf{P}_{1} \diamond \mathbf{P}_{2} \diamond \mathbf{P}_{3} \diamond \mathbf{P}_{4} \diamond \mathbf{P}_{5}
$$

and (B.29) becomes

$$
\mathfrak{J}=\left(\mathbf{P}_{1} \diamond \mathbf{P}_{2} \diamond \mathbf{P}_{3} \diamond \mathbf{P}_{4} \diamond \mathbf{P}_{5}\right)^{H}\left(\mathbf{P}_{1} \diamond \mathbf{P}_{2} \diamond \mathbf{P}_{3} \diamond \mathbf{P}_{4} \diamond \mathbf{P}_{5}\right)
$$

\section{B.5 Equivalence of Wideband Error Bound with and without Noise Variance in Parameter set}

Consider the parametric prediction assumption upon which the derivation of the bound is based where the channel parameters are estimated from the noisy estimates $(\hat{\mathbf{H}}(p, q))$ and the parameter estimates used for the extrapolation. The predicted sample can therefore be denoted as $\tilde{\mathbf{H}}(p, q)=$ $f(\theta, \phi, \tau, \nu, p, q)$ independent of the estimate of $\sigma^{2}$. We will now show that including $\sigma^{2}$ in $\Theta$ does not change the expression for the bound.

Consider the parameter set in (8.102) with $\sigma^{2}$ included

$$
\tilde{\boldsymbol{\Theta}}=\left[\begin{array}{lllll}
\sigma^{2} & \boldsymbol{\theta}_{1} & \boldsymbol{\theta}_{2} & \cdots & \boldsymbol{\theta}_{Z}
\end{array}\right]
$$

Using Bangs formula as given in (8.12), the FIM can be expressed as

$$
\tilde{\mathfrak{J}}(\tilde{\boldsymbol{\Theta}})=\left[\begin{array}{cc}
\frac{K N M Q}{\sigma^{4}} & \mathbf{0}^{T} \\
\mathbf{0} & \mathfrak{J}(\boldsymbol{\Theta})
\end{array}\right]
$$

where the first element of (B.34) have been obtained from the first term of (8.12) with $\mathbf{C}=\sigma^{2} \mathbf{I}$ and $\mathbf{J}(\boldsymbol{\Theta})$ is given in (8.115). Now using the vector form of the CRB for functions of parameters, the mean square error bound (MSEB) becomes

$$
\operatorname{MSEB}(p, q)=\sum_{n=1}^{N} \sum_{m=1}^{M} \frac{\partial \tilde{h}(n, m, p, q)}{\partial \tilde{\boldsymbol{\Theta}}} \tilde{\mathbf{J}}(\tilde{\boldsymbol{\Theta}}) \frac{\partial \tilde{h}(n, m, p, q)}{\partial \tilde{\boldsymbol{\Theta}}}^{H}
$$


where $\tilde{h}(n, m, p, q)$ denotes the estimated, interpolated or predicted channel. Since the channel reconstruction does not utilize the estimate of the noise variance, the partial derivative in (B.37) can be expressed as

$$
\begin{aligned}
\frac{\partial \tilde{h}(n, m, p, q)}{\partial \tilde{\boldsymbol{\Theta}}} & =\left[\begin{array}{ll}
\frac{\partial \tilde{h}(n, m, p, q)}{\partial \sigma^{2}} & \frac{\partial \tilde{h}(n, m, p, q)}{\partial \boldsymbol{\Theta}}
\end{array}\right] \\
& =\left[\begin{array}{ll}
0 & \frac{\partial \tilde{h}(n, m, p, q)}{\partial \boldsymbol{\Theta}}
\end{array}\right]
\end{aligned}
$$

Substituting (B.34) and (B.36) into (B.37) yields

$$
\operatorname{MSEB}(p, q)=\sum_{n=1}^{N} \sum_{m=1}^{M} \frac{\partial \tilde{h}(n, m, p, q)}{\partial \boldsymbol{\Theta}} \mathbf{J}(\boldsymbol{\Theta}) \frac{\partial \tilde{h}(n, m, p, q)}{\partial \boldsymbol{\Theta}}^{H}
$$

which is the same as the expression in (8.119) (for the case without $\sigma^{2}$ in the parameter set).

\section{B.6 Simplifications for Narrowband Asymptotic FIM}

Consider the expression for the FIM in (8.89) and assume that $K, N$, and/or $M$ are large such that

$$
\sum_{p=0}^{P-1} \sum_{n=1}^{N} \sum_{m=1}^{M} h \approx K N M \mathbb{E}[h]
$$


Using (8.89) and (8.90)-(8.94), the diagonal entries of the FIM are obtained as

$$
\begin{aligned}
{\left[\mathfrak{J}\left(\boldsymbol{\theta}_{p}\right)\right]_{11} } & =\frac{2 N M K}{\sigma^{2}} \\
{\left[\mathfrak{J}\left(\boldsymbol{\theta}_{p}\right)\right]_{22} } & =\frac{2 N M K}{\sigma^{2}} \\
{\left[\mathfrak{J}\left(\boldsymbol{\theta}_{p}\right)\right]_{33} } & =\frac{M K N(N-1)(2 N-1)\left|\alpha_{p}\right|^{2}}{3 \sigma^{2}} \\
{\left[\mathfrak{J}\left(\boldsymbol{\theta}_{p}\right)\right]_{44} } & =\frac{N K M(M-1)(2 M-1)\left|\alpha_{p}\right|^{2}}{3 \sigma^{2}} \\
{\left[\mathfrak{J}\left(\boldsymbol{\theta}_{p}\right)\right]_{55} } & =\frac{N M K(K-1)(2 K-1) \Delta^{2}\left|\alpha_{p}\right|^{2}}{3 \sigma^{2}}
\end{aligned}
$$

Following a similar approach, the off-diagonal entries can be shown to be

$$
\begin{aligned}
& {\left[\mathfrak{J}\left(\boldsymbol{\theta}_{p}\right)\right]_{12}=\left[\mathbf{I}\left(\boldsymbol{\theta}_{p}\right)\right]_{21}=0} \\
& {\left[\mathfrak{J}\left(\boldsymbol{\theta}_{p}\right)\right]_{13}=\left[\mathbf{I}\left(\boldsymbol{\theta}_{p}\right)\right]_{31}=\frac{M K N(N-1) \mathfrak{I}\left(\alpha_{p}\right)}{\sigma^{2}}} \\
& {\left[\mathfrak{J}\left(\boldsymbol{\theta}_{p}\right)\right]_{14}=\left[\mathbf{I}\left(\boldsymbol{\theta}_{p}\right)\right]_{41}=\frac{N K M(M-1) \mathfrak{I}\left(\alpha_{p}\right)}{\sigma^{2}}} \\
& {\left[\mathfrak{J}\left(\boldsymbol{\theta}_{p}\right)\right]_{15}=\left[\mathbf{I}\left(\boldsymbol{\theta}_{p}\right)\right]_{51}=\frac{N M K(K-1) \Delta \mathfrak{I}\left(\alpha_{p}\right)}{\sigma^{2}}} \\
& {\left[\mathfrak{J}\left(\boldsymbol{\theta}_{p}\right)\right]_{23}=\left[\mathbf{I}\left(\boldsymbol{\theta}_{p}\right)\right]_{32}=\frac{M K N(N-1) \mathfrak{R}\left(\alpha_{p}\right)}{\sigma^{2}}} \\
& {\left[\mathfrak{J}\left(\boldsymbol{\theta}_{p}\right)\right]_{24}=\left[\mathbf{I}\left(\boldsymbol{\theta}_{p}\right)\right]_{42}=\frac{N K M(M-1) \mathfrak{R}\left(\alpha_{p}\right)}{\sigma^{2}}} \\
& {\left[\mathfrak{J}\left(\boldsymbol{\theta}_{p}\right)\right]_{25}=\left[\mathbf{I}\left(\boldsymbol{\theta}_{p}\right)\right]_{52}=\frac{N M K(K-1) \Delta \mathfrak{R}\left(\alpha_{p}\right)}{\sigma^{2}}} \\
& {\left[\mathfrak{J}\left(\boldsymbol{\theta}_{p}\right)\right]_{34}=\left[\mathbf{I}\left(\boldsymbol{\theta}_{p}\right)\right]_{43}=\frac{N M K(N-1)(M-1)\left|\alpha_{p}\right|^{2}}{2 \sigma^{2}}} \\
& {\left[\mathfrak{J}\left(\boldsymbol{\theta}_{p}\right)\right]_{35}=\left[\mathbf{I}\left(\boldsymbol{\theta}_{p}\right)\right]_{53}=-\frac{N M K(N-1)(K-1) \Delta\left|\alpha_{p}\right|^{2}}{2 \sigma^{2}}} \\
& {\left[\mathfrak{J}\left(\boldsymbol{\theta}_{p}\right)\right]_{45}=\left[\mathbf{I}\left(\boldsymbol{\theta}_{p}\right)\right]_{54}=-\frac{N M K(M-1)(K-1) \Delta\left|\alpha_{p}\right|^{2}}{2 \sigma^{2}}}
\end{aligned}
$$




\section{B.7 Simplifications for Wideband Asymptotic FIM}

Consider the expression for the FIM in (8.89) and assume that $Q, K, N$, and/or $M$ are large such that

$$
\sum_{q=0}^{Q-1} \sum_{p=0}^{P-1} \sum_{n=1}^{N} \sum_{m=1}^{M} h \approx Q P N M \mathbb{E}[h]
$$

Using (8.89) and (8.107)-(8.112), the diagonal entries of the FIM are obtained as

$$
\begin{aligned}
& {[\mathfrak{J}]_{11}=\frac{2 K Q N M}{\sigma^{2}} \mathbb{E}[1]} \\
& {[\mathfrak{J}]_{22}=\frac{2 Q K N M}{\sigma^{2}} \mathbb{E}[1]} \\
& {[\mathfrak{J}]_{33}=\frac{2}{\sigma^{2}}\left(\sum_{q=0}^{Q-1} \sum_{k=0}^{K-1} \sum_{n=1}^{N} \sum_{m=1}^{M}(n-1)^{2}\left|\alpha_{p}\right|^{2}\right)} \\
& {[\mathfrak{J}]_{44}=\frac{2}{\sigma^{2}}\left(\sum_{q=0}^{Q-1} \sum_{k=0}^{K-1} \sum_{n=1}^{N} \sum_{m=1}^{M}(m-1)^{2}\left|\alpha_{p}\right|^{2}\right)} \\
& {[\mathfrak{J}]_{55}=\frac{2}{\sigma^{2}}\left(\sum_{q=0}^{Q-1} \sum_{k=0}^{K-1} \sum_{n=1}^{N} \sum_{m=1}^{M}\left(k U_{t}\right)^{2}\left|\alpha_{p}\right|^{2}\right)} \\
& {[\mathfrak{J}]_{66}=\frac{2}{\sigma^{2}}\left(\sum_{q=0}^{Q-1} \sum_{k=0}^{K-1} \sum_{n=1}^{N} \sum_{m=1}^{M}\left(q U_{f}\right)^{2}\left|\alpha_{p}\right|^{2}\right)}
\end{aligned}
$$


Using (B.38) in (B.55) yields

$$
\begin{aligned}
{[\mathfrak{J}]_{11} } & =\frac{2 Q K N M}{\sigma^{2}} \\
{[\mathfrak{J}]_{22} } & =\frac{2 Q K N M}{\sigma^{2}} \\
{[\mathfrak{J}]_{33} } & =\frac{2}{\sigma^{2}}\left(M K Q\left(\sum_{n=1}^{N}(n-1)^{2}\right) \mathbb{E}\left[\left|\alpha_{p}\right|^{2}\right]\right) \\
{[\mathfrak{J}]_{44} } & =\frac{2}{\sigma^{2}}\left(N K Q\left(\sum_{m=1}^{M}(m-1)^{2}\right) \mathbb{E}\left[\left|\alpha_{p}\right|^{2}\right]\right) \\
{[\mathfrak{J}]_{55} } & =\frac{2}{\sigma^{2}}\left(N M Q\left(\sum_{k=0}^{K-1}\left(k U_{\mathrm{t}}\right)^{2}\right) \mathbb{E}\left[\left|\alpha_{p}\right|^{2}\right]\right) \\
{[\mathfrak{J}]_{66} } & =\frac{2}{\sigma^{2}}\left(N M K\left(\sum_{q=0}^{Q-1}\left(q U_{\mathrm{f}}\right)^{2}\right) \mathbb{E}\left[\left|\alpha_{p}\right|^{2}\right]\right)
\end{aligned}
$$

Using the identity

$$
\sum_{a=1}^{A} a^{2}=\frac{A(A+1)(2 A+1)}{6}
$$

equations (B.56) becomes

$$
\begin{aligned}
& {[\mathfrak{J}]_{33}=\frac{2}{\sigma^{2}}\left(\frac{M K Q N(N-1)(2 N-1)}{6} \mathbb{E}\left[\left|\alpha_{p}\right|^{2}\right]\right)} \\
& {[\mathfrak{J}]_{44}=\frac{2}{\sigma^{2}}\left(\frac{N K Q M(M-1)(2 M-1)}{6} \mathbb{E}\left[\left|\alpha_{p}\right|^{2}\right]\right)} \\
& {[\mathfrak{J}]_{55}=\frac{2}{\sigma^{2}}\left(\frac{N M Q K(K-1)(2 K-1) U_{\mathrm{t}}^{2}}{6} \mathbb{E}\left[\left|\alpha_{p}\right|^{2}\right]\right)} \\
& {[\mathfrak{J}]_{66}=\frac{2}{\sigma^{2}}\left(\frac{N M K Q(Q-1)(2 Q-1) U_{\mathrm{f}}^{2}}{6} \mathbb{E}\left[\left|\alpha_{p}\right|^{2}\right]\right)}
\end{aligned}
$$

We now use our assumption that the complex amplitude is $\alpha_{p} \sim \mathcal{C N}(0,1)$, such that

$$
\begin{aligned}
\mathbb{E}\left[\left|\alpha_{p}\right|^{2}\right] & =1 \\
\mathbb{E}\left[\alpha_{p}\right] & =0
\end{aligned}
$$


and apply (B.67) in (B.63) to obtain

$$
\begin{aligned}
& {[\mathfrak{J}]_{33}=\frac{2}{\sigma^{2}}\left(\frac{M K Q N(N-1)(2 N-1)}{6}\right)} \\
& {[\mathfrak{J}]_{44}=\frac{2}{\sigma^{2}}\left(\frac{N K Q M(M-1)(2 M-1)}{6}\right)} \\
& {[\mathfrak{J}]_{55}=\frac{2}{\sigma^{2}}\left(\frac{N M Q K(K-1)(2 K-1) U_{\mathrm{t}}^{2}}{6}\right)} \\
& {[\mathfrak{J}]_{66}=\frac{2}{\sigma^{2}}\left(\frac{N M K Q(Q-1)(2 Q-1) U_{\mathrm{f}}^{2}}{6}\right)}
\end{aligned}
$$

Since $N, M, Q, K>1$, the approximations $A-1 \approx A$ and $2 A-1 \approx 2 A$ can be used to simplify (B.68) to

$$
\begin{aligned}
& {[\mathfrak{J}]_{33}=\frac{N M K Q}{\sigma^{2}}\left(\frac{2 N^{2}}{3}\right)} \\
& {[\mathfrak{J}]_{44}=\frac{N M K Q}{\sigma^{2}}\left(\frac{2 M^{2}}{3}\right)} \\
& {[\mathfrak{J}]_{55}=\frac{N M K Q}{\sigma^{2}}\left(\frac{2 K^{2} U_{\mathrm{t}}^{2}}{3}\right)} \\
& {[\mathfrak{J}]_{66}=\frac{N M K Q}{\sigma^{2}}\left(\frac{2 Q^{2} U_{\mathrm{f}}^{2}}{3}\right)}
\end{aligned}
$$

The off-diagonal entries of the FIM are obtained following the same procedure. 


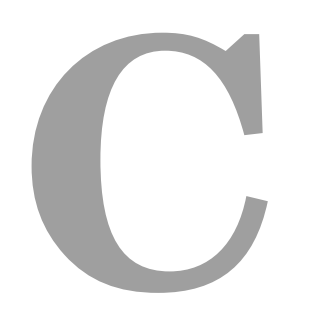

\section{System Level Performance Evaluation}

$\mathrm{T}$

HIS section outlines our preliminary results and ongoing research on the effects of outdated CSI and gains of using our prediction methods in multiuser MIMO systems.

\section{C.1 System Model}

We consider the downlink of a multiuser MIMO system as illustrated in Fig. C.1. We assume that the BS has an N-element ULA and that there are $Z$ users each with $M_{z} \geq 1$ antenna elements. Denoting the transmitted signal and precoding matrix for the $z$ th user as $\mathbf{s}_{z}$ and $\mathbf{W}_{z}$, respectively, the received signal at the $z$ th user can be expressed as

$$
\mathbf{r}_{z}=\mathbf{H}_{z} \mathbf{W}_{z} \mathbf{s}_{z}+\sum_{i=1, i \neq z}^{Z} \mathbf{H}_{z} \mathbf{W}_{i} \mathbf{s}_{i}+\mathbf{n}_{z}
$$




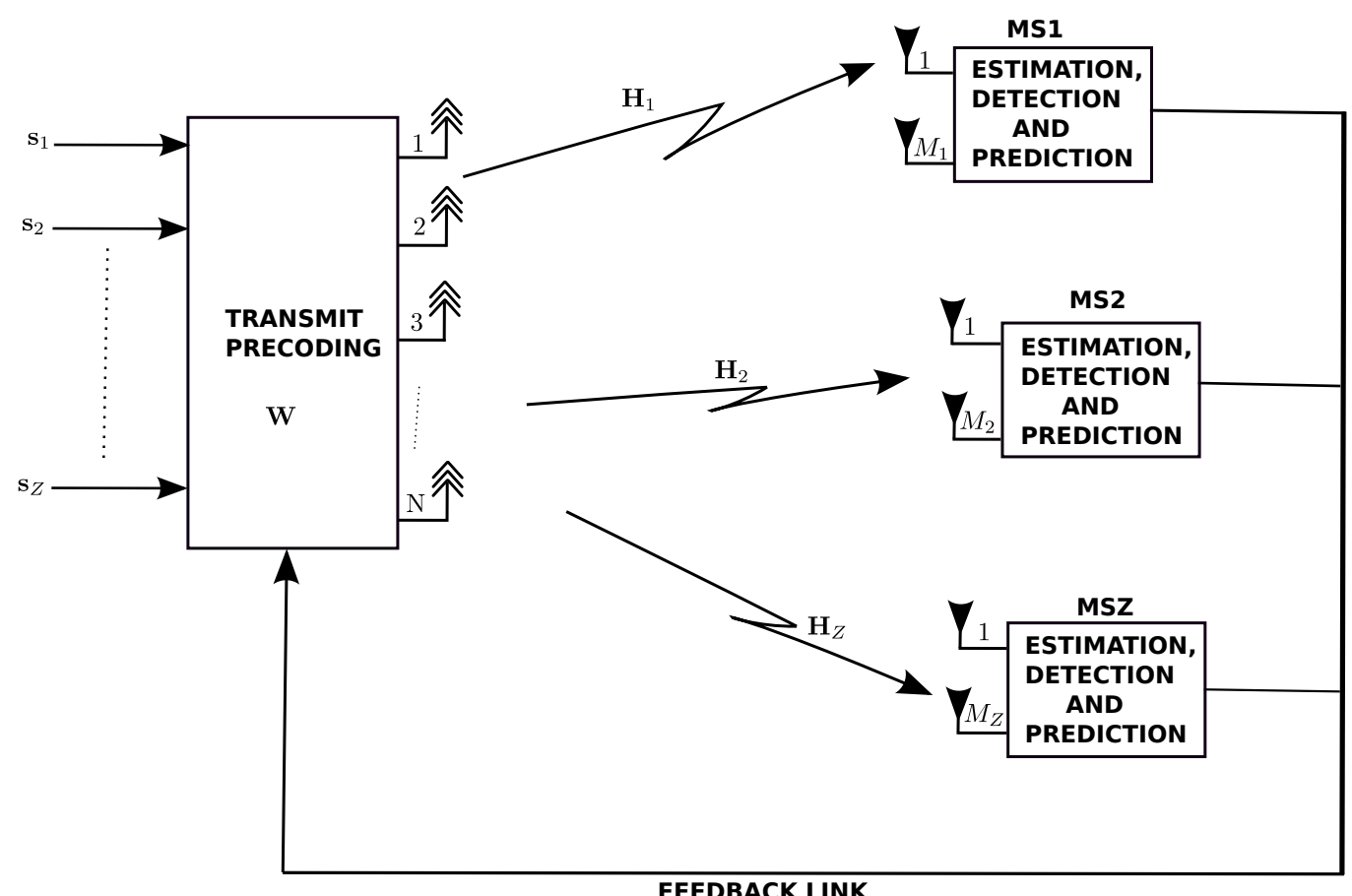

Figure C.1: Block diagram of a multiuser Mobile MIMO System.

where $\mathbf{n}_{z}$ is the zero mean Gaussian distributed receive noise vector at the $z$ th receiver. $\mathbf{n}_{z}$ is assumed to have a variance $\sigma^{2}$ per antenna. We assume an FDD transmission where the CSI is estimated at each of the mobile stations and fed back to the BS via a dedicated feedback link as shown in Fig. C.1. Assuming that the CSI is known perfectly at the BS, the Signalto-Interference-plus-Noise Ratio (SINR) at the $z$ th user is given by [161]

$$
\operatorname{SINR}_{z}=\frac{\left\|\mathbf{H}_{z} \mathbf{W}_{z}\right\|^{2}}{M_{z} \sigma^{2}+\sum_{i=1, i \neq z}^{Z}\left\|\mathbf{H}_{z} \mathbf{W}_{i}\right\|^{2}}
$$

and the corresponding rate is

$$
R_{z}=\log _{2}\left(1+\operatorname{SINR}_{z}\right)
$$

The capacity/sum rate of the multiuser system is thus

$$
R=\sum_{z=1}^{Z} R_{z}
$$


In reality the CSI at the transmitter have some imperfections resulting from receiver mobility, channel estimation and quantization. Our aim in this work is to investigate the effect of using delayed CSI on the performance of linear precoding schemes and evaluate the performance gains from using our prediction schemes. In Fig. C.2, we illustrate the effect of feedback delay

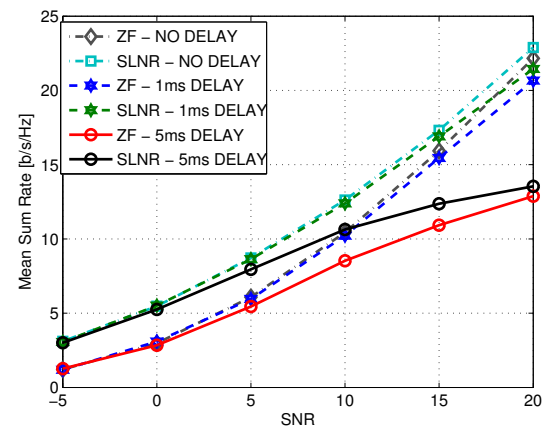

(a) $v=5 \mathrm{kmph}$

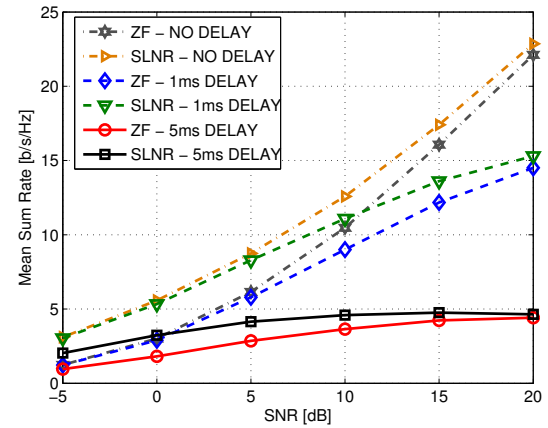

(c) $v=20 \mathrm{kmph}$

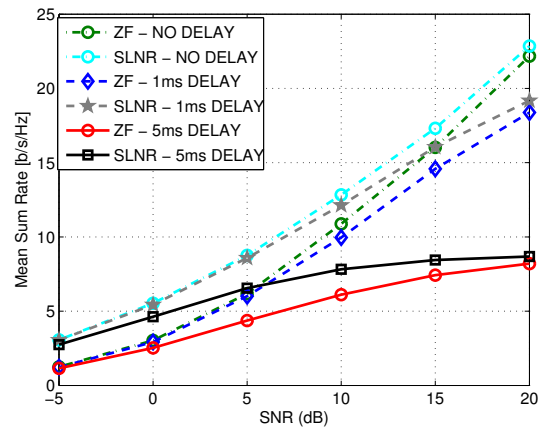

(b) $v=10 \mathrm{kmph}$

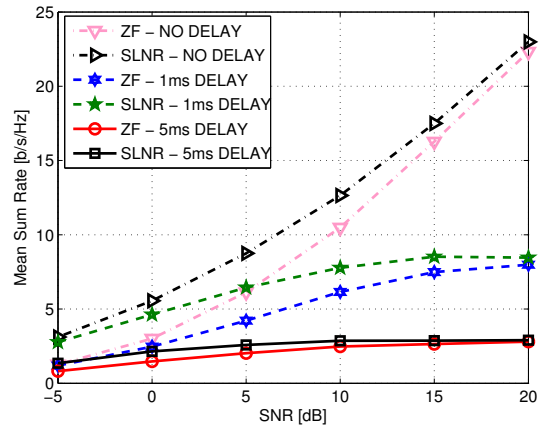

(d) $v=50 \mathrm{kmph}$

Figure C.2: Mean Sum Rate versus SNR for different mobile velocity with perfect and delayed CSI.

on the performance of ZF [78] and SLNR [161] transmit beamformers. We plot the sum rate CDFs at $\mathrm{SNR}=30 \mathrm{~dB}$ for a multiuser MISO system with four users and four transmit antennas at different feedback delay values. We observe that the performance of the two precoding methods degrades even for delay values as low as $1 \mathrm{~ms}$. The figures also shows that for some delay 
value, the performance degradation increases with increased mobile velocity. For instance, with a feedback delay of $1 \mathrm{~ms}$, the mean sum rate decreased by approximately $1 \mathrm{~b} / \mathrm{s} / \mathrm{Hz}$ and $15 \mathrm{~b} / \mathrm{s} / \mathrm{Hz}$ at mobile velocity values of $5 \mathrm{kmph}$ and $50 \mathrm{kmph}$, respectively.

\section{C.2 Performance of Transmit Beamforming with Predicted CSIT}

In this proposed study, we will study the performance gains of using our prediction methods with precoded multiuser MISO and MIMO systems. Since the mobile terminals has only a single antenna in the MISO systems, we will utilize only the MSSM and RSSM based methods to predict the CSI. However, all methods will be applied to the MIMO systems. 


\section{Bibliography}

[1] Spatial channel model for multiple input multiple output (MIMO) simulations. Tech. rep., 3GGP, TR 25.996, V7.0.0. 2007-06.

[2] Spatial channel model for multiple input multiple output (MIMO) simulations. Tech. rep., 3GPP TR 25.996 V6.1.0, sep. 2003.

[3] IST-WINNER II Deliverable 1.1.2 v.1.2, WINNER II channel models. Tech. rep., IST-WINNER2, 2007.

[4] IEEE P802.11p/D9.0: Part 11: Wireless LAN Medium Access Control (MAC) and Physical Layer (PHY) Specifications: Amendment: Wireless Access in Vehicular Environments (WAVE). Tech. rep., IEEE, 2009.

[5] Part 16: Air Interface for Broadband Wireless Access Systems. IEEE Standard for local and metropolitan area networks. IEEE, May 2009.

[6] WINNER + final channel models. Tech. rep., Wireless World Initiative New Radio, 2010.

[7] Abed-Meraim, K., And HuA, Y. A least-squares approach to joint schur decomposition. In IEEE ICASSP (1998), vol. 4, pp. 2541-2544.

[8] Aboutajdine, D., Adib, A., And Meziane, A. Fast adaptive algorithms for AR parameters estimation using higher order statistics. IEEE Transactions on Signal Processing 44, 8 (Aug 1996), 1998-2009. 
[9] Acosta, G., Tokuda, K., And Ingram, M.-A. Measured joint Doppler-delay power profiles for vehicle-to-vehicle communications at 2.4 GHz. In IEEE Global Telecommunications Conference (2004), vol. 6, pp. 3813-3817 Vol.6.

[10] Adeogun, R., Teal, P. D., And Dmochowski, P. A. Parametric Channel Prediction for Narrowband Mobile MIMO Systems Using Spatio-Temporal Correlation Analysis. In IEEE VTC Fall (2013).

[11] Adeogun, R. O., Teal, P. D., And Dmochowski, P. A. Asymptotic Error Bounds on Prediction of Narrowband MIMO Wireless Channels. IEEE Signal Processing Letters, 1103 - 1107.

[12] Adeogun, R. O., Teal, P. D., And Dmochowski, P. A. Methods for Prediction of Narrowband MIMO Channels in 3D Propagation Environments. IEEE Transactions on Antenna and Propagation.

[13] Adeogun, R. O., Teal, P. D., And Dmochowski, P. A. Long range parametric channel prediction for narrowband MIMO systems with joint parameter estimation. In Seventh International Conference on Signal Processing and Communication Systems (Dec 2013), pp. 575580 .

[14] Adeogun, R. O., Teal, P. D., And Dmochowski, P. A. Parametric Channel Prediction for Narrowband Mobile MIMO Systems Using Spatio-Temporal Correlation Analysis. In IEEE Vehicular Technology Conference Fall (Sep 2013).

[15] Adeogun, R. O., Teal, P. D., And Dmochowski, P. A. Extrapolation of MIMO Mobile to Mobile Wireless Channels Using Parametric Model Based Prediction. IEEE Transactions on Vehicular Techniology pp (2014). 
[16] Adeogun, R. O., Teal, P. D., And Dmochowski, P. A. Novel Algorithm for Prediction of Wideband Mobile MIMO Wireless Channels. In IEEE International Conference on Communications (June 2014).

[17] Adeogun, R. O., Teal, P. D., And Dmochowski, P. A. Parametric Channel Prediction for Narrowband MIMO Systems Using Polarized Antenna Arrays. In IEEE Vehicular Technology Conference Spring (May 2014).

[18] Adeogun, R. O., Teal, P. D., And Dmochowski, P. A. Parametric Schemes for Prediction of Wideband MIMO Wireless Channels. IEEE Transactions on Signal Processing (2014 (submitted)).

[19] Adeogun, R. O., Teal, P. D., And Dmochowski, P. A. An Asymptotic Bound on Estimation and Prediction of MIMO-OFDM Wireless Channels. IEEE Transactions on Vehicular Technology (2014(in review)).

[20] Akaike, H. A new look at the statistical model identification. IEEE Transactions on Automatic Control 19 (1974), 716-723.

[21] Akki, A. S. Statistical properties of mobile-to-mobile land communication channels. IEEE Transactions on Vehicular Technology 43 (1994), 826-831.

[22] AkKi, A. S., ANd HABER, F. A statistical model of mobile-to-mobile land communication channel. IEEE Transactions on Vehicular Technology 35 (1986), 2-7.

[23] Almers, P., Bonek, E., Burr, A., Czink, N., Debbah, M., Degli-esposti, V., Hofstetter, H., Kyosti, P., Laurenson, D., Matz, G., Molisch, A. F., Oestges, C., and Ozcelik, H. Survey of channel and radio propagation models for wireless MIMO systems. EURASIP Journal on Wireless Communications and Networking (2007). 
[24] Andersen, J., Jensen, J., Jensen, S., And Frederisen, F. Prediction of future fading based on past measurements. In IEEE Vehicular Technology Conference (1999), vol. 1, pp. 151-155.

[25] Anderson, B., And Moore, J. Optimal Filtering. Englewood Cliffs, New Jersey: Prentice-Hall, 1979.

[26] Ar, E. T., And Telatar, I. E. Capacity of Multi-antenna Gaussian Channels. European Transactions on Telecommunications 10 (1999), 585-595.

[27] Arredondo, A., Dandekar, K. R., And Xu, G. Vector channel modeling and prediction for the improvement of downlink received power. IEEE Trans. on Comm. 50, 7 (2002), 1121-1129.

[28] Arulampalam, M., Maskell, S., Gordon, N., and Clapp, T. A tutorial on particle filters for online nonlinear/non-gaussian bayesian tracking. IEEE Transactions on Signal Processing 50, 2 (Feb 2002), $174-188$.

[29] Asplund, H., Glazunov, A. A., Molisch, A. F., Pedersen, K. I., And Steinbauer, M. The COST 259 Directional Channel Model - Part II: Macrocells. IEEE Transactions on Wireless Communications 5, 12 (2006), 3434-3450.

[30] Baddour, K., And Beaulieu, N. Autoregressive modeling for fading channel simulation. IEEE Transactions on Wireless Communications 4, 4 (2005), 1650-1662.

[31] Baddour, K., And Beaulieu, N. Improved Pilot-Assisted Prediction of Unknown Time-Selective Rayleigh Channels. In IEEE International Conference on Communications (ICC) (2006), vol. 11, pp. 51925199. 
[32] Baddour, K. E., Squires, C. C., And Willink, T. J. Mobile Channel Prediction with Application to Transmitter Antenna Selection for Alamouti Systems. In VTC Fall (2006), pp. 1-6.

[33] Badeau, R., Richard, G., and David, B. Adaptive ESPRIT algorithm based on the PAST subspace tracker. In IEEE International Conference on Acoustics, Speech, and Signal Processing (April 2003), vol. 6, pp. VI-229-32.

[34] BARABell, A. Improving the resolution performance of eigenstructure-based direction-finding algorithms. In IEEE International Conference on Acoustics, Speech, and Signal Processing (Apr 1983), vol. 8, pp. 336-339.

[35] Barbarossa, S., and Scaglione, A. Theoretical bounds on the estimation and prediction of multipath time-varying channels. In IEEE International Conference on Acoustics, Speech, and Signal Processing (2000), vol. 5, pp. 2545-2548.

[36] Baum, D., Hansen, J., and Salo, J. An interim channel model for beyond-3G systems: extending the 3GPP spatial channel model (SCM). In IEEE 61st Vehicular Technology Conference (May 2005), vol. 5, pp. 3132-3136.

[37] Bingham, J. Multicarrier modulation for data transmission: An idea whose time has come. IEEE Communication Magazine 28 (May 1990), 5.

[38] Bunse-Gerstner, A., Byers, R., And Mehrmann, V. Numerical methods for simultaneous diagonalization. SIAM Journal of Matrix Analysis and Applications 14 (1993), 927-949.

[39] CADzow, J. A. An extrapolation procedure for band-limited signals. IEEE Transactions on Acoustics, Speech and Signal Processing 27, 1 (Feb 1979), 4-12. 
[40] Catreux, S., Erceg, V., Gesbert, D., And Heath, R. Adaptive modulation and MIMO coding for broadband wireless data networks. IEEE Communications Magazine 40, 6 (2002), 108-115.

[41] Chae, C.-B., Forenza, A., JR., R. W. H., McKay, M. R., And Collings, I. B. Adaptive MIMO transmission techniques for broadband wireless communication systems [Topics in Wireless Communications]. IEEE Communications Magazine 48, 5 (2010), 112-118.

[42] Chang, J. Adaptive codebook-based channel prediction and interpolation for multiuser multiple-input multiple-outputâĂŞorthogonal frequency division multiplexing systems. IET Communications 6 (February 2012), 281-288(7).

[43] Chang, J., Lu, I.-T., And Li, Y. Adaptive Codebook Based Channel Prediction and Interpolation for Multiuser MIMO-OFDM Systems. In IEEE International Conference on Communications (2011), pp. 1-5.

[44] Chang, J., Lu, I.-T., And Li, Y. Efficient Adaptive Double Codebook Based CSI Prediction for SU/MU MIMO-OFDM Systems. In IEEE Global Telecommunications Conference (Dec 2011), pp. 1-6.

[45] Chen, M., Ekman, T., And Viberg, M. New approaches for channel prediction based on sinusoidal modeling. EURASIP Journal of Applied Signal Processing 2007, 1 (Jan 2007), 197-197.

[46] Chen, M., And Viberg, M. Long-range channel prediction based on nonstationary parametric modeling. IEEE Transactions on Signal Processing 57, 2 (Feb 2009), 622-634.

[47] Cheng, X., Yao, Q., Wang, C.-X., Ai, B., Stüber, G. L., Yuan, D., AND JiAO, B. An Improved Parameter Computation Method for a MIMO V2V Rayleigh Fading Channel Simulator Under Non-Isotropic Scattering Environments. IEEE Communications Letters 17, 2 (2013), 265-268. 
[48] Chirag S. Patel and Gordon L. Stüber and Thomas G. PRATT. Simulation of Rayleigh-faded mobile-to-mobile communication channels. IEEE Transactions on Communications 53, 11 (2005), 1876-1884.

[49] Chiurtu, N., Rimoldi, B., And Telatar, I. On the capacity of multi-antenna Gaussian channels. In IEEE International Symposium on Information Theory (2001), pp. 53-58.

[50] Correia, L. M. Mobile Broadband Multimedia Networks: Techniques, Models and Tools for $4 G$. Academic Press.

[51] Cramer, H. Mathematical Methods of Statistics. Princeton University Press, Princeton, NJ, USA, 1946.

[52] Czink, N., And Oestges, C. The COST 273 MimO Channel Model: Three Kinds of Clusters. In IEEE 10th International Symposium on Spread Spectrum Techniques and Applications (Aug 2008), pp. 282-286.

[53] Czink, N., And Oestges, C. The COST 273 MimO Channel Model: Three Kinds of Clusters. In IEEE 10th International Symposium on Spread Spectrum Techniques and Applications (2008), pp. 282-286.

[54] Dahlman, E., Parkvall, S., Skold, J., And Beming, P. 3G Evolution, Second Edition: HSPA and LTE for Mobile Broadband, 2 ed. Academic Press, 2008.

[55] Dao, M.-T., NGuyen, V.-A., Im, Y.-T., Park, S.-O., And Yoon, G. 3D Polarized Channel Modeling and Performance Comparison of MIMO Antenna Configurations With Different Polarizations. IEEE Transactions on Antennas and Propagation 59, 7 (2011), 2672-2682.

[56] Duel-Hallen, A. Fading Channel Prediction for Mobile Radio Adaptive Transmission Systems. Proceedings of the IEEE 95, 12 (2007), 2299-2313. 
[57] Duel-Hallen, A., Hu, S., And Hallen, H. Long range prediction of fading signals: Enabling adaptive transmission for mobile radio channels. IEEE Signal Processing Magazine 17 (2000), 62-75.

[58] Edt, D. G. Mobile WiMAX - Part I: A Technical Overview and Performance Evaluation. WiMAX Forum, 2006.

[59] Eiceg, V., Sampath, H., And Catreux-Erceg, S. Dualpolarization versus single-polarization MIMO channel measurement results and modeling. IEEE Transactions on Wireless Communications 5, 1 (Jan 2006), 28-33.

[60] Ekman, T. Prediction of Mobile Radio Channels - Modeling and Design. PhD thesis, 2002.

[61] Falahati, S., Svensson, A., Ekman, T., And Sternad, M. Adaptive Modulation Systems for Predicted Wireless Channels. IEEE Transactions on Communication 52, 2 (2004), 307-316.

[62] Foschini, G. J., And Gans, M. J. On limits of wireless communications in a fading environment when using multiple antennas. Wireless Personal Communications 6 (1998), 311-335.

[63] Fu, T., AND GaO, X. Simultaneous diagonalization with similarity transformation for non-defective matrices. In IEEE ICASSP (2006), vol. 4, pp. IV-IV.

[64] Fung, C. Y., And Chan, S. Estimation of fast fading channel in impulse noise environment. In IEEE International Symposium on Circuits and Systems (2002), vol. 4, pp. IV-497-IV-500 vol.4.

[65] Gast, M. S. 802.11 Wireless Networks: The Definitive Guide, Second Edition. O'Reilly Media, Inc., 2005. 
[66] Gerchberg, R. W. Superresolution through error function extrapolation. In Sixth Multidimensional Signal Processing Workshop (Sep 1989), pp. 185-.

[67] Gifford, S., Bergstrom, C., And Chuprun, S. Adaptive and linear prediction channel tracking algorithms for mobile OFDM-MIMO applications. In IEEE Military Communications Conference (Oct 2005), pp. 1298-1302 Vol. 2.

[68] Godana, B., And Ekman, T. Linear prediction of time-varying MIMO systems using Givens rotations. In IEEE Workshop on Signal Processing Advances in Wireless Communications (2011), pp. 371-375.

[69] Goldsmith, A. Wireless Communications. Cambridge University Press, New York, NY, USA, 2005.

[70] Gong, J., Hayes, J., And Soleymani, M. The effect of antenna physics on fading correlation and the capacity of multielement antenna systems. IEEE Transactions on Vehicular Technology 56, 4 (July 2007), 1591-1599.

[71] Guillaud, M., And Slock, D. T. M. A specular approach to MIMO frequency-selective channel tracking and prediction. In IEEE 5th Workshop on Signal Processing Advances in Wireless Communications (July 2004), pp. 59-63.

[72] Guncavdi, S., And Duel-Hallen, A. Performance analysis of space-time transmitter diversity techniques for WCDMA using long range prediction. IEEE Transactions on Wireless Communications 4 , 1 (Jan 2005), 40-45.

[73] HaArdt, M., AND Nossek, J. Unitary ESPRIT: how to obtain increased estimation accuracy with a reduced computational burden. IEEE Transactions on Signal Processing 43, 5 (May 1995), 1232-1242. 
[74] HaArdt, M., And Nossek, J. Simultaneous schur decomposition of several nonsymmetric matrices to achieve automatic pairing in multidimensional harmonic retrieval problems. IEEE Transactions on Signal Processing 46, 1 (1998), 161-169.

[75] Haardt, M., Roemer, F., And Del Galdo, G. Higher-order svdbased subspace estimation to improve the parameter estimation accuracy in multidimensional harmonic retrieval problems. IEEE Transactions on Signal Processing 56, 7 (July 2008), 3198-3213.

[76] Hallen, H., Duel-Hallen, A., Hu, S., Yang, T.-S., And Lei, M. A physical model for wireless channels to provide insights for long range prediction. In MILCOM 2002. Proceedings (Oct 2002), vol. 1, pp. $627-631$.

[77] Hassanien, A., Vorobyov, S. A., and Gershman, A. B. Moving Target Parameters Estimation in Noncoherent MIMO Radar Systems. IEEE Transactions on Signal Processing 60, 5 (2012), 2354-2361.

[78] Haustein, T., Von Helmolt, C., Jorswieck, E., Jungnickel, V., And POHL, V. Performance of MIMO systems with channel inversion. In IEEE 55th Vehicular Technology Conference (2002), vol. 1, pp. 35-39 vol.1.

[79] Heddergott, R., Bernhard, U., And Fleury, B. Stochastic radio channel model for advanced indoor mobile communication systems. In The 8th IEEE International Symposium on Personal, Indoor and Mobile Radio Communications (Sep 1997), vol. 1, pp. 140-144 vol.1.

[80] Heidari, A., Khandani, A. K., And McAvoy, D. Adaptive modeling and long-range prediction of mobile fading channels. IET communications (Jan 2010).

[81] Hogstad, B., Patzold, M., Youssef, N., And Kim, D. A Mimo Mobile-To-Mobile Channel Model: Part II - The Simulation Model. In 
IEEE 16th International Symposium on Personal, Indoor and Mobile Radio Communications (2005), vol. 1, pp. 562-567.

[82] Hu, A., Lv, T., GaO, H., Zhang, Z., And Yang, S. An ESPRIT-Based Approach for 2-D Localization of Incoherently Distributed Sources in Massive MIMO Systems. IEEE Journal of Selected Topics in Signal Processing PP, 99 (2014).

[83] HuA, Y., AND SARKAR, T. Matrix pencil method for estimating parameters of exponentially damped/undamped sinusoids in noise. IEEE Transactions on Acoustics, Speech and Signal Processing 38, 5 (May 1990), 814-824.

[84] Huang, L., Long, T., MaO, E., And So, H.-C. MMSE-based MDL method for robust estimation of number of sources without eigendecomposition. IEEE Transactions on Signal Processing 57, 10 (2009), 4135-4142.

[85] Huang, L., Long, T., Mao, E., So, H. C., And Member, S. MMSE-Based MDL Method for Accurate Source Number Estimation. IEEE Signal Processing Letters 16, 9 (sept 2009), 798-801.

[86] ILTIS, R. Joint estimation of PN code delay and multipath using the extended Kalman filter. IEEE Transactions on Communications 38, 10 (Oct 1990), 1677-1685.

[87] Inoue, T., And Heath, JR., R. W. Grassmannian Predictive Coding for delayed limited feedback MIMO systems. In Proceedings of the 4 th annual Allerton conference on Communication, control, and computing (Piscataway, NJ, USA, 2009), Allerton'09, IEEE Press, pp. 783-788.

[88] Ivrlac, M., Utschick, W., And Nossek, J. Fading correlations in wireless MIMO communication systems. IEEE Journal on Selected Areas in Communications 21, 5 (June 2003), 819-828. 
[89] JAin, A., And RANGAnATh, S. Extrapolation algorithms for discrete signals with application in spectral estimation. IEEE Transactions on Acoustics, Speech and Signal Processing 29, 4 (1981), 830-845.

[90] Jakobsen, M., Laugesen, K., Manchon, C., Kirkelund, G., Rom, C., And Fleury, B. Parametric Modeling and Pilot-Aided Estimation of the Wireless Multipath Channel in OFDM Systems. In IEEE International Conference on Communications (May 2010), pp. $1-6$.

[91] JARINOvA, D. On autoregressive model order for long-range prediction of fast fading wireless channel. Telecommunication Systems 52, 3 (2013), 1533-1539.

[92] Jensen, M., And Wallace, J. A review of antennas and propagation for MIMO wireless communications. IEEE Transactions on Antennas and Propagation 52, 11 (Nov 2004), 2810-2824.

[93] K, H. J., AND .H, W. J. Sinusoidal modeling and prediction of fast fading. In IEEE Global Telecommunications Conference (1998), pp. 892-897.

[94] Kalman, R. E. A New Approach to Linear Filtering and Prediction Problems. Transactions of the ASME - Journal of Basic Engineering, 82 (Series D) (1960), 35-45.

[95] Kanai, H., Abe, M., And Kido, K. Accurate autoregressive spectrum estimation at low signal-to-noise ratio using a phase matching technique. IEEE Transactions on Acoustics, Speech and Signal Processing 35, 9 (Sep 1987), 1264-1272.

[96] KaY, S., And Marple, S.L., J. Spectrum analysis: A modern perspective. Proceedings of the IEEE 69, 11 (Nov 1981), 1380-1419. 
[97] KAY, S. M. Fundamentals of statistical signal processing: estimation theory. Prentice-Hall, Inc., Upper Saddle River, NJ, USA, 1993.

[98] Khare, K., And George, N. Sampling theory approach to prolate spheroidal wavefunctions. Journal of Physics 36, 39 (2003), 1001110021.

[99] Khrwat, A., Sharif, B. S., Tsimenidis, C., Boussakta, S., And Al-DweIK, A. J. Channel prediction for limited feedback precoded MIMO-OFDM systems. In IEEE International Symposium on Signal Processing and Information Technology (Dec 2009), pp. 195-200.

[100] Khrwat, A. S. Channel prediction for limited feedback precoded MIMO-OFDM systems. In ICT (2012), IEEE, pp. 1-6.

[101] Kim, K. J., Pun, M.-O., And Iltis, R. A. Channel prediction for limited feedback precoded MIMO-OFDM systems over time-varying fading channels. In CISS (2008), pp. 972-977.

[102] Komninakis, C., Fragouli, C., Sayed, A., And Wesel, R. Multi-input multi-output fading channel tracking and equalization using Kalman estimation. IEEE Transactions on Signal Processing 50, 5 (May 2002), 1065-1076.

[103] Kountouris, M., SÄlzer, T., And Gesbert, D. Scheduling for multiuser MIMO downlink channels with ranking-based feedback. EURASIP Journal on Advances in Signal Processing 2008 (Jan 2008), 131:1-131:14.

[104] Kovacs, I., Eggers, P. C. F., Olesen, K., and Petersen, L. Investigations of outdoor-to-indoor mobile-to-mobile radio communication channels. In IEEE 56th Vehicular Technology Conference (2002), vol. 1 , pp. $430-434$ vol.1. 
[105] Kozono, S., Tsuruhara, T., And Sakamoto, M. Base station polarization diversity reception for mobile radio. Vehicular Technology, IEEE Transactions on 33, 4 (Nov 1984), 301-306.

[106] Kyosti, P., Meinila, J., Hentila, L., Zhao, X., Jamsa, T., Schneider, C., Narandzic, M., Milojevic, M., Hong, A., Ylitalo, J., Holappa, V.-M., Alatossava, M., Bultitude, R., De Jong, Y., And Rautiainen, T. WinneR II Channel Models. Tech. rep., Sep 2007.

[107] Lande, S. B., Helonde, J. B., Pande, R., and Pathak, S. S. Adaptive Subcarrier and Bit Allocation for Downlink OFDMA System with Proportional Fairness. CoRR abs/1111.2160 (2011).

[108] Larsen, M., Swindlehurst, A., And Svantesson, T. A Performance Bound for Interpolation of MIMO-OFDM Channels. In Fortieth Asilomar Conference on Signals, Systems and Computers (Oct 2006), pp. 1801-1805.

[109] Larsen, M., Swindlehurst, L., and Svantesson, T. A Performance Bound for MIMO-OFDM Channel Estimation and Prediction. 2008, pp. 141-145.

[110] Larsen, M. D., Swindlehurst, A. L., And Svantesson, T. Performance bounds for MIMO-OFDM channel estimation. IEE Transactions on Signal Processing 57, 5 (May 2009), 1901-1916.

[111] LEE, A. Centrohermitian and skew-centrohermitian matrices. Linear Algebra and its Applications 29, 0 (1980), 205-210. Special Volume Dedicated to Alson S. Householder.

[112] Lee, D., Kashyap, R., And Madan, R. Robust decentralized direction-of-arrival estimation in contaminated noise. IEEE Transactions on Acoustics, Speech and Signal Processing 38, 3 (Mar 1990), 496-505. 
[113] Li, F.-K., Held, D., Curlander, J., And Wu, C. Doppler parameter estimation for spaceborne synthetic-aperture radars. IEEE Transactions on Geoscience and Remote Sensing GE-23, 1 (Jan 1985), $47-56$.

[114] Li, G., AND StÜBer, G. Orthogonal Frequency Division Multiplexing for Wireless Communications. Signals and Communication Technology. Springer Science+Business Media, 2006.

[115] Li, J., Conan, J., ANd Pierre, S. Joint Estimation of Channel Parameters for MIMO Communication Systems. In 2nd International Symposium on Wireless Communications (sept 2005), pp. 22-26.

[116] Li, L., Li, H., Yu, H., YAng, B., AND Hu, H. A New Algorithm for MIMO Channel Tracking Based on Kalman Filter. In IEEE Wireless Communications and Networking Conference (March 2007), pp. 164168.

[117] Liang, D., Guanghan, X., And Hao, L. Prediction of fast fading mobile radio channels in wideband communication systems. In IEEE Global Telecommunications Conference (2001), pp. 3287-3291.

[118] Liu, J., And Liu, X. Time-varying channel identification and prediction in OFDM systems using 2-D frequency estimation. In IEEE conference on Military communications (Piscataway, NJ, USA, 2006), MILCOM'06, IEEE Press, pp. 2777-2783.

[119] Liu, L., Feng, H., Yang, T., And Hu, B. MiMO-OFDM Wireless Channel Prediction by Exploiting Spatial-Temporal Correlation. IEEE Transactions on Wireless Communications 13, 1 (January 2014), 310319.

[120] Liu, Q., Zhou, S., And Giannakis, G. Efficient bandwidth utilization guaranteeing QoS over adaptive wireless links. In IEEE Global Telecommunications Conference (Nov 2004), vol. 4, pp. 2684-2688. 
[121] Love, D. J., Heath-Jr., R. W., Lau, V. K. N., Gesbert, D., RAO, B. D., AND Andrews, M. An overview of limited feedback in wireless communication systems. IEEE Journal on Selected Areas in Communications 26, 8 (2008), 1341-1365.

[122] Lyman, R., And Sikora, A. Prediction of fading envelopes with diffuse spectra. In IEEE International Conference on Acoustics, Speech, and Signal Processing (2005), vol. 3, pp. iii/753-iii/756.

[123] Maddah-Ali, M., And Tse, D. Completely stale transmitter channel state information is still very useful. Information Theory, IEEE Transactions on 58, 7 (July 2012), 4418-4431.

[124] Malik, W., Allen, B., And Edwards, D. Fade depth scaling with channel bandwidth. Electronics Letters 43, 24 (Nov 2007), 1371-1372.

[125] Manolakis, K., Jaeckel, S., Jungnickel, V., And Braun, V. Channel prediction by doppler-delay analysis and benefits for base station cooperation. In IEEE rrth Vehicular Technology Conference (VTC) (Dresden, Germany, jun 2013).

[126] Mathews, C. P., HaArdt, M., and Zoltowski, M. D. Performance analysis of closed form, ESPRIT based 2-D Angle Estimator for rectangular arrays. IEEE Signal Processing Letters (1996).

[127] Maurer, J., Fugen, T., Schafer, T., And Wiesbeck, W. A new inter-vehicle communications (IVC) channel model. In IEEE Vehicular Technology Conference (2004), vol. 1, pp. 9-13 Vol. 1.

[128] Maurer, J., Fugen, T., And Wiesbeck, W. Narrow-band measurement and analysis of the inter-vehicle transmission channel at 5.2 GHz. In IEEE 55th Vehicular Technology Conference (2002), vol. 3, pp. $1274-1278$ vol.3. 
[129] Maurer, J., Schafer, T., And Wiesbeck, W. A realistic description of the environment for inter-vehicle wave propagation modelling. In IEEE 54th Vehicular Technology Conference (2001), vol. 3, pp. 1437-1441 vol.3.

[130] McCloud, M., And Scharf, L. A new subspace identification algorithm for high-resolution DOA estimation. IEEE Transactions on Antennas and Propagation 50, 10 (Oct 2002), 1382-1390.

[131] Miao, H., Juntti, M. J., And Yu, K. 2-D Unitary ESPRIT Based Joint AOA and AOD Estimation for MIMO System. In IEEE 17th International Symposium on Personal, Indoor and Mobile Radio Communications (2006), IEEE, pp. 1-5.

[132] Milosavluevic, M., Veinovic, M., And Kovacevic, B. Estimation of nonstationary AR model using the weighted recursive least square algorithm. In International Conference on Acoustics, Speech, and Signal Processing (May 1995), vol. 2, pp. 1432-1435 vol.2.

[133] Min, C., Chang, N., Cha, J., And Kang, J. MiMO-OFDM Downlink Channel Prediction for IEEE802.16e Systems Using Kalman Filter. In IEEE Wireless Communications and Networking Conference (March 2007), pp. 942-946.

[134] Molisch, A., Tufvesson, F., Karedal, J., And MecklenBRAUKER, C. Propagation aspects of vehicle-to-vehicle communications - an overview. In IEEE Radio and Wireless Symposium, 2009 (2009), pp. 179-182.

[135] Molisch, A. F., Asplund, H., Heddergott, R., Steinbauer, M., And Zwick, T. The COST259 Directional Channel Model Part I: Overview and Methodology, 2006.

[136] Narandzic, M., Schneider, C., And Thoma. WINnER wideband MIMO system-level channel model, comparison with other refer- 
ence models. In Internationales Wissenschartliches Kolloquium (2009), vol. 54 .

[137] Narandzic, M., Schneider, C., Thoma, R., Jamsa, T., Kyosti, P., AND ZHAO, X. Comparison of SCM, SCME, and WINNER Channel Models. In IEEE 65th Vehicular Technology Conference (April 2007), pp. 413-417.

[138] Narandzic, M., Schneider, C., Thoma, R., Jamsa, T., Kyosti, P., AND ZHAO, X. Comparison of SCM, SCME, and WINNER Channel Models. In IEEE 65th Vehicular Technology Conference (April 2007), pp. 413-417.

[139] Nguyen, H. T., Andersen, J. B., And Pedersen, G. F. Capacity and performance of MIMO systems under the impact of feedback delay. In PIMRC (2004), pp. 53-57.

[140] Nguyen, H. T., Leus, G., And Khaled, N. Precoder and decoder prediction in time-varying MIMO channels. In 1st IEEE International Workshop on Computational Advances in Multi-Sensor Adaptive Processing (Dec 2005), pp. 153-156.

[141] N.Kikuma, H.Kikuchi, and N.Inagaki. Pairing of Estimates Using Mean Eigenvalue Decomposition in Multi-Dimensional Unitary ESPRIT. IEICE Trans J82-B, 11 (Nov 1999), 2202-2207.

[142] Oien, G. E., Holm, H., And Hole, K. J. Impact of channel prediction on adaptive coded modulation performance in Rayleigh fading. IEEE Transactions on Vehicular Technology 53 (2004), 758-769.

[143] Okino, K., Nakayama, T., Joko, S., Kusano, Y., And Kimura, $\mathrm{S}$. Direction based beamspace MIMO channel prediction with ray cancelling. In IEEE PIMRC (2008), pp. 1-5. 
[144] Ozcelik, H., Herdin, M., Weichselberger, W., Wallace, J., AND BONEK, E. Deficiencies of 'kronecker' mimo radio channel model. Electronics Letters 39, 16 (Aug 2003), 1209-1210.

[145] PApoulis, A. A new algorithm in spectral analysis and band limited signal extrapolation. IEEE Transactions on Circuits and Systems CAS22 (Sep 1975), 735-742.

[146] Patzold, M., Hogstad, B., Youssef, N., And Kim, D. A MimO Mobile-To-Mobile Channel Model: Part I - The Reference Model. In IEEE 16th International Symposium on Personal, Indoor and Mobile Radio Communications (2005), vol. 1, pp. 573-578.

[147] Patzold, M., And Hogstad, B. O. Classes of sum-of-sinusoids rayleigh fading channel simulators and their stationary and ergodic properties: part I. WSEAS Transactions on Mathematics 5, 2 (2006), 222 .

[148] Paulraj, A., NABAR, R., AND Gore, D. Introduction to SpaceTime Wireless Communications, 1st ed. Cambridge University Press, New York, NY, USA, 2008.

[149] Phelps, C. I., And Buehrer, R. M. A simplified technique for adaptive MIMO, modulation, and coding. In 4th international conference on Radio and wireless symposium (Piscataway, NJ, USA, 2009), RWS'09, IEEE Press, pp. 539-542.

[150] Pop, M., AND Beaulieu, N. Statistical investigation of sum-ofsinusoids fading channel simulators. In IEEE Global Telecommunications Conference (1999), vol. 1A, pp. 419-426.

[151] Prakash, S., And Mcloughlin, I. Effects of Channel Prediction for Transmit Antenna Selection With Maximal-Ratio Combining in Rayleigh Fading. IEEE Transactions on Vehicular Technology 60, 6 (July 2011), 2555-2568. 
[152] Pronkis, J. G. Digital Communications, fourth ed. McGraw-Hill, aug 2001.

[153] RAO, C. R. Information and the accuracy attainable in the estimation of statistical parameters. Bulletin of the Calcutta Mathematical Society (1945), 81-89.

[154] Rappaport, T. S. Wireless Communications: Principles and Practice, 1st ed. IEEE Press, Piscataway, NJ, USA, 1996.

[155] Real, E., Tufts, D., And Cooley, J. Two algorithms for fast approximate subspace tracking. IEEE Transactions on Signal Processing 47, 7 (Jul 1999), 1936-1945.

[156] Rhee, D., Hwang, H. G., Sang, Y. J., And Kim, K. S. Multiuser adaptive transmission technique for time-varying frequency-selective fading channels. Signal Processing 88, 8 (Aug 2008), 2095-2107.

[157] Roemer, F., HaArdt, M., and Del Galdo, G. Analytical performance assessment of multi-dimensional matrix- and tensor-based esprit-type algorithms. IEEE Transactions on Signal Processing 62, 10 (May 2014), 2611-2625.

[158] Roy, R., And Kailath, T. Estimation of signal parameters via rotational invariance techniques. IEEE Trans on Acoustics, Speech, and Signal Processing 37 (Jul 1989), 984-995.

[159] Roy, R., And Kailath, T. Signal processing part II. Springer-Verlag New York, Inc., New York, NY, USA, 1990, ch. ESPRIT-estimation of signal parameters via rotational invariance techniques, pp. 369-411.

[160] Sabri, M. S., And SteenaArt, W. An Approach to Band-Limited Extrapolation The Extrapolation Matrix. IEEE Transactions on Circuit and Systems 25, 2 (Feb 1978), 74-78. 
[161] SAdek, M., TArighat, A., And SAyed, A. A leakage-based precoding scheme for downlink multi-user mimo channels. Wireless Communications, IEEE Transactions on 6, 5 (May 2007), 1711-1721.

[162] Sameni, R., Jutten, C., And Shamsollahi, M. A deflation procedure for subspace decomposition. IEEE Transactions on Signal Processing 58, 4 (April 2010), 2363-2374.

[163] Sampath, H., Talwar, S., Tellado, J., Erceg, V., And PAulraj, A. A fourth-generation MIMO-OFDM broadband wireless system: design, performance, and field trial results. IEEE Communications Magazine 40, 9 (Sep 2002), 143-149.

[164] SARKAR, T., AND PEREIRA, O. Using the matrix pencil method to estimate the parameters of a sum of complex exponentials. IEEE Antennas and Propagation Magazine 37, 1 (Feb 1995), 48-55.

[165] Saunders, S. R., And Aragon, A. Antennas and Propagation for Wireless Communication Systems. Wiley \& Sons, 2007.

[166] Sayeed, A., Sendonaris, A., And Aazhang, B. Multiuser detection in fast-fading multipath environments. IEEE Journal on Selected Areas in Communications 16, 9 (Dec 1998), 1691-1701.

[167] Schafhuber, D., And Matz, G. MMSE and adaptive prediction of time-varying channels for OFDM systems. IEEE Transactions on Wireless Communications 4, 2 (2005), 593-602.

[168] Schmidt, R. Multiple emitter location and signal parameter estimation. IEEE Transactions on Antennas and Propagation 34, 3 (Mar 1986), 276-280.

[169] Semmelrodt, S., And Kattenbach, R. Investigation of different fading forecast schemes for flat fading radio channels. In IEEE 58th Vehicular Technology Conference (2003), vol. 1, pp. 149-153 Vol.1. 
[170] SeybolD, J. Introduction to RF Propagation. John Wiley \& Sons, 2005.

[171] Shafi, M., Zhang, M., Moustakas, A. L., Smith, P. J., Molisch, A. F., Tufvesson, F., And Simon, S. H. Polarized MIMO Channels in 3D: Models, Measurements and Mutual Information. IEEE Journal on Selected Areas Communication 24 (2006), 514527.

[172] Shannon, C. E. A mathematical theory of communication. Bell system technical journal 27 (1948).

[173] Sharif, M., And Abeysekera, S. Efficient wideband sonar parameter estimation using a single slice of radon-ambiguity transform. In IEEE International Conference on Acoustics, Speech, and Signal Processing (March 2005), vol. 5, pp. v/605-v/608.

[174] Sharif, M., And Abeysekera, S. Efficient Active Sonar Parameter Estimation Using Linear FM Signals via Hermite Decompositions. In OCEANS 2006 - Asia Pacific (May 2006), pp. 1-5.

[175] Sharma, P., And Chandra, K. Prediction of State Transitions in Rayleigh Fading Channels. IEEE Transactions on Vehicular Technology 56, 2 (March 2007), 416-425.

[176] Shirani-Mehr, H., Liu, D. N., And Caire, G. Channel State Prediction, Feedback and Scheduling for a Multiuser MIMO-OFDM Downlink. CoRR abs/0811.4630 (2008).

[177] Shiu, D., Foschini, G., Gans, M., And Kahn, J. Fading correlation and its effect on the capacity of multielement antenna systems. IEEE Transactions on Communications 48, 3 (Mar 2000), 502-513. 
[178] Slepian, D. Prolate spheroidal wave functions, fourier analysis, and uncertainty-v: The discrete case. Bell Syst. Tech. Journal 5\%, 5 (MayJun. 1978), 1371-1430.

[179] Slepian, D., AND Pollak, H. Prolate Spheroidal Wave Functions, Fourier Analysis and Uncertainty - I. Bell System Technical Journal 40 (1961).

[180] Soltanian-Zadeh, H., And Yagle, A. A fast algorithm for extrapolation of discrete band-limited signals. In IEEE International Conference on Acoustics, Speech, and Signal Processing (April 1993), vol. 3, pp. 591-594 vol.3.

[181] SRIvastava, A. Performance bounds for subspace estimation in array signal processing. In Ninth IEEE SP Workshop on Statistical Signal and Array Processing (Sep 1998), pp. 140-143.

[182] Steinbauer, M., Molisch, A., And Bonek, E. The doubledirectional radio channel. IEEE Antennas and Propagation Magazine 43, 4 (Aug 2001), 51-63.

[183] Stoica, P., And Arye, N. MUSIC, maximum likelihood, and Cramer-Rao bound. IEEE Transactions on Acoustics, Speech and Signal Processing 37, 5 (May 1989), 720-741.

[184] StoicA, P., And Moses, R. L. Introduction to spectral analysis, vol. 1. Prentice hall New Jersey:, 1997.

[185] Strobach, P. Bi-iteration SVD subspace tracking algorithms. IEEE Transactions on Signal Processing 45, 5 (May 1997), 1222-1240.

[186] Strobach, P. The fast householder Bi-SVD subspace tracking algorithm. Signal Processing 88, 11 (Nov 2008), 2651-2661. 
[187] Sun, D., LIU, Z., MA, S., AND Yi, K. Channel prediction using iaa-based spectral estimation in precoded TDD-MIMO systems. IEEE Communications Letters 17, 4 (2013), 701-704.

[188] Sun, D., Liu, Z., MA, S., AND Yi, K. Channel prediction using iaa-based spectral estimation in precoded TDD-MIMO systems. IEEE Communications Letters 17, 4 (2013), 701-704.

[189] Svantesson, T., And Swindlehurst, A. A performance bound for prediction of MIMO channels. IEEE Transactions on Signal Processing 54, 2 (Oct 2006), 520-529.

[190] Teal, P., And Vaughan, R. Simulation and performance bounds for real-time prediction of the mobile multipath channel. In IEEE Workshop on Statistical Signal Processing Proceedings (2001), pp. 548551.

[191] Truong, K. T., And JR., R. W. H. Effects of channel aging in massive MIMO systems. Journal of Communications and Networks 15, 4 (Aug. 2013), 338-351.

[192] Tse, D., And Viswanath, P. Fundamentals of Wireless Communications. 2004.

[193] Vanderpypen, J., And Schumacher, L. MiMO Channel Prediction using ESPRIT based Techniques. In IEEE PIMRC (2007).

[194] Vatalaro, F., And Forcella, A. Doppler spectrum in mobile-tomobile communications in the presence of three-dimensional multipath scattering. IEEE Transactions on Vehicular Technology 46, 1 (1997), 213-219.

[195] Vaughan, R., Teal, P., And Raich, R. Short-term mobile channel prediction using discrete scatterer propagation model and subspace sig- 
nal processing algorithms. In IEEE Vehicular Technology Conference (2000), vol. 2, pp. 751-758.

[196] Visuri, S., OjA, H., And Koivunen, V. Robust subspace DOA estimation for wireless communications. In IEEE 51st Vehicular Technology Conference (2000), vol. 3, pp. 2551-2555.

[197] Wang, A., LiU, L., AND ZhAng, J. Low complexity direction of arrival (DoA) estimation for 2D massive MIMO systems. In IEEE Globecom Workshops (Dec 2012), pp. 703-707.

[198] Wang, J., ZhaO, J., And GaO, X. Modeling and analysis of polarized MIMO channels in 3D propagation environment. In IEEE 21st International Symposium on Personal Indoor and Mobile Radio Communications (2010), pp. 319-323.

[199] Wax, M., AND Kailath, T. Detection of signals by information theoretic criteria. IEEE Transactions on Acoustics, Speech and Signal Processing 33, 2 (1985), 387-392.

[200] Weichselberger, W., Herdin, M., Ozcelik, H., And Bonek, E. A stochastic MIMO channel model with joint correlation of both link ends. IEEE Transactions on Wireless Communications 5, 1 (Nov 2006), 90-100.

[201] Williams, D. Comparison of AIC and MDL to the minimum probability of error criterion. In IEEE Sixth SP Workshop on Statistical Signal and Array Processing (Oct 1992), pp. 114-117.

[202] Williams, M. I. Y., Dickins, G., Kennedy, R. A., Pollock, T. S., And Abhayapala, T. D. Novel Scheme for Spatial Extrapolation of Multipath. pp. 784-787. 
[203] Wong, C. Y., Cheng, R., Lataief, K., And Murch, R. Multiuser OFDM with adaptive subcarrier, bit, and power allocation. IEEE Journal on Selected Areas in Communications 17, 10 (1999), 1747-1758.

[204] Wong, I., And Evans, B. Exploiting Spatio-Temporal Correlations in MIMO Wireless Channel Prediction. In IEEE Global Telecommunications Conference (Nov 2006), pp. 1-5.

[205] Wong, I. C., And Evans, B. L. Sinusoidal Modeling and Adaptive Channel Prediction in Mobile OFDM Systems. IEEE Transactions on Signal Processing 56, 4 (2008), 1601-1615.

[206] Wu, W.-R., And Chen, P.-C. Adaptive AR modeling in white Gaussian noise. IEEE Transactions on Signal Processing 45, 5 (May 1997), 1184-1192.

[207] Xia, P., Member, S., Zhou, S., and Giannakis, G. B. Adaptive MIMO-OFDM Based on Partial Channel State Information. IEEE Transactions on Signal Processing 52 (2004), 202-213.

[208] Xu, G., Roy, R.H., I., And Kailath, T. Detection of number of sources via exploitation of centro-symmetry property. IEEE Transactions on Signal Processing 42, 1 (Jan 1994), 102-112.

[209] YAnfei, G., AND Zishu, H. MIMO channel tracking based on Kalman filter and MMSE-DFE. In International Conference on Communications, Circuits and Systems (May 2005), vol. 1, pp. 223-226.

[210] YANG, B. Subspace tracking based on the projection approach and the recursive least squares method. In IEEE International Conference on Acoustics, Speech, and Signal Processing (April 1993), vol. 4, pp. 145148. 
[211] YANG, B. An extension of the PASTd algorithm to both rank and subspace tracking. IEEE Signal Processing Letters 2, 9 (Sept 1995), $179-182$.

[212] YAnG, B. Projection approximation subspace tracking. IEEE Transactions on Signal Processing 43, 1 (Jan 1995), 95-107.

[213] Yang, B., And Bahme, J. F. Rotation-based RLS algorithms: unified derivations, numerical properties, and parallel implementations. IEEE Transactions on Signal Processing 40, 5 (May 1992), 1151-1167.

[214] Yang, B., Letaief, K. B., Cheng, R. S., And CaO, Z. Channel estimation for OFDM transmission in multipath fading channels based on parametric channel modeling. IEEE Transactions on Communications 49, 3 (aug 2002), 467-479.

[215] Yang, J., Tin, N., And Khandani, A. Adaptive modulation and coding in 3G wireless systems. In IEEE 56th Vehicular Technology Conference (2002), vol. 1, pp. 544-548.

[216] Yao, D., Camargo, A., And Czylwik, A. Adaptive MimO Transmission Scheme for Spatially Correlated Broadband BICM-OFDM Systems. In IEE Vehicular Technology Conference Fall (2008), pp. 1-5.

[217] ZajIC, A. G. Estimation of Velocities in Mobile-to-Mobile Wireless Fading Channels. In VTC Fall (2011), pp. 1-5.

[218] ZajIC, A. G. Estimation of Mobile Velocities and Direction of Movement in Mobile-to-Mobile Wireless Fading Channels. IEEE T. Vehicular Technology 61, 1 (2012), 130-139.

[219] Zajic, A. G., And Stuber, G. L. Simulation models for mimo mobile-to-mobile channels. In Proceedings of the 2006 IEEE conference on Military communications (2006), MILCOM'06, pp. 1905-1911. 
[220] Zajic, A. G., And Stüber, G. L. A Three-Dimensional MIMO Mobile-to-Mobile Channel Model. In WCNC (2007), pp. 1883-1887.

[221] Zajic, A. G., And Stüber, G. L. A Three Dimensional Parametric Model for Wideband MIMO Mobile-to-Mobile Channels. In GLOBECOM (2007), pp. 3760-3764.

[222] Zajic, A. G., And Stüber, G. L. Maximum Likelihood Method for MIMO Mobile-to-Mobile Channel Parameter Estimation. In GLOBECOM (2008), pp. 3969-3973.

[223] Zemen, T., Hofstetter, H., And Steinbock, G. Successive slepian subspace projection in time and frequency for time-variant channel estimation. In 14TH IST Mobile and Wireless Communication Summit (IST) (2005).

[224] Zemen, T., And Mecklenbrauker, C. Time-variant channel estimation using discrete prolate spheroidal sequences. IEEE Transactions on Signal Processing 53, 9 (Sept 2005), 3597-3607.

[225] Zemen, T., Mecklenbrauker, C., And Fleury, B. Timevariant channel prediction using time-concentrated and band-limited sequences. In IEEE International Conference on Communications (2006), vol. 12, pp. 5660-5665.

[226] Zhang, L., Jin, Z., Chen, W., And Zhang, X. An improved adaptive channel prediction for MIMO-OFDM systems. In Third International Conference on Communications and Networking in China (Aug 2008), pp. 1008-1012.

[227] Zhou, S., And Giannakis, G. How accurate channel prediction needs to be for transmit-beamforming with adaptive modulation over Rayleigh MIMO channels? IEEE Transactions on Wireless Communications 3, 4 (2004), 1285-1294. 
[228] Zhu, Y., Liu, L., Wang, A., Sayana, K., and Zhang, J. DoA estimation and capacity analysis for 2D active massive MIMO systems.

In IEEE International Conference on Communications (June 2013), pp. $4630-4634$. 